\title{
Diabetes care and the introduction of a new standard in the Netherlands : a closer look from the perspectives of health care professionals and patients
}

Citation for published version (APA):

Raaijmakers, L. G. M. (2014). Diabetes care and the introduction of a new standard in the Netherlands : a closer look from the perspectives of health care professionals and patients. [Doctoral Thesis, Maastricht University]. Datawyse / Universitaire Pers Maastricht. https://doi.org/10.26481/dis.20141210lr

Document status and date:

Published: 01/01/2014

DOI:

10.26481/dis.20141210Ir

Document Version:

Publisher's PDF, also known as Version of record

Please check the document version of this publication:

- A submitted manuscript is the version of the article upon submission and before peer-review. There can be important differences between the submitted version and the official published version of record.

People interested in the research are advised to contact the author for the final version of the publication, or visit the DOI to the publisher's website.

- The final author version and the galley proof are versions of the publication after peer review.

- The final published version features the final layout of the paper including the volume, issue and page numbers.

Link to publication

\footnotetext{
General rights rights.

- You may freely distribute the URL identifying the publication in the public portal. please follow below link for the End User Agreement:

www.umlib.nl/taverne-license

Take down policy

If you believe that this document breaches copyright please contact us at:

repository@maastrichtuniversity.nl

providing details and we will investigate your claim.
}

Copyright and moral rights for the publications made accessible in the public portal are retained by the authors and/or other copyright owners and it is a condition of accessing publications that users recognise and abide by the legal requirements associated with these

- Users may download and print one copy of any publication from the public portal for the purpose of private study or research.

- You may not further distribute the material or use it for any profit-making activity or commercial gain

If the publication is distributed under the terms of Article $25 \mathrm{fa}$ of the Dutch Copyright Act, indicated by the "Taverne" license above, 


\section{DIABETES CARE AND THE INTRODUCTION OF A NEW STANDARD IN THE NETHERLANDS}

A closer look from the perspectives of health care professionals and patients 


\section{Colophon}

Production: Datawyse | Universitaire Pers Maastricht

(C) Lieke Raaijmakers, Maastricht 2014

ISBN 9789461593771

The research presented in this dissertation was performed within the NUTRIM School for Nutrition, Toxicology and Metabolism, Maastricht University.

The work was conducted as part of the Dutch National Action program Diabetes, with a grant from the Diabetes Federation (project number 2010.105.1356). 


\title{
DIABETES CARE AND THE INTRODUCTION OF A NEW STANDARD IN THE NETHERLANDS
}

\author{
A closer look from the perspectives of \\ health care professionals and patients
}

\author{
PROEFSCHRIFT
}

ter verkrijging van de graad van doctor aan de Universiteit Maastricht, op gezag van de Rector Magnificus, Prof. dr. L.L.G. Soete, volgens het besluit van het College van Decanen,

in het openbaar te verdedigen, op woensdag 10 december 2014 om 12.00 uur

door

Lieke Geertruda Maria Raaijmakers

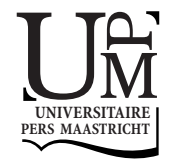




\section{Promotores}

Prof. dr. N.K. de Vries

Prof. dr. S.P.J. Kremers

\section{Beoordelingscommissie}

Prof. dr. H. van de Mheen (voorzitter)

Prof. dr. L. Maes (Universiteit Gent)

Prof. dr. G.D.E.M. van der Weijden

Dr. D.M. Hettinga (Diabetes Fonds)

Dr. C. Hoving 


\section{Contents}

$\begin{array}{lll}\text { Chapter } 1 \text { General Introduction } & 7\end{array}$

Chapter 2 Perceptions of Dutch health care professionals regarding the 21 Care Standard for diabetes

Chapter 3 Perceived facilitators and barriers in diabetes care: a qualitative study among health care professionals in the Netherlands

Chapter 4 Correlates of perceived self-care activities and diabetes control among Dutch type 1 and type 2 diabetics

Chapter 5 Mastery and perceived autonomy support are correlates of Dutch diabetes patients' self-management and quality of life

Chapter 6 The implementation of the Netherlands Diabetes Federation Care Standard for Diabetes anno 2013

Chapter 7 The implementation of the National Action program Diabetes in the Netherlands: lessons learned

Chapter 8 General discussion

Valorization addendum

References

Summary

Samenvatting

Dankwoord

Curriculum Vitae

Publication list 



\section{Chapter 1}

\section{General introduction}

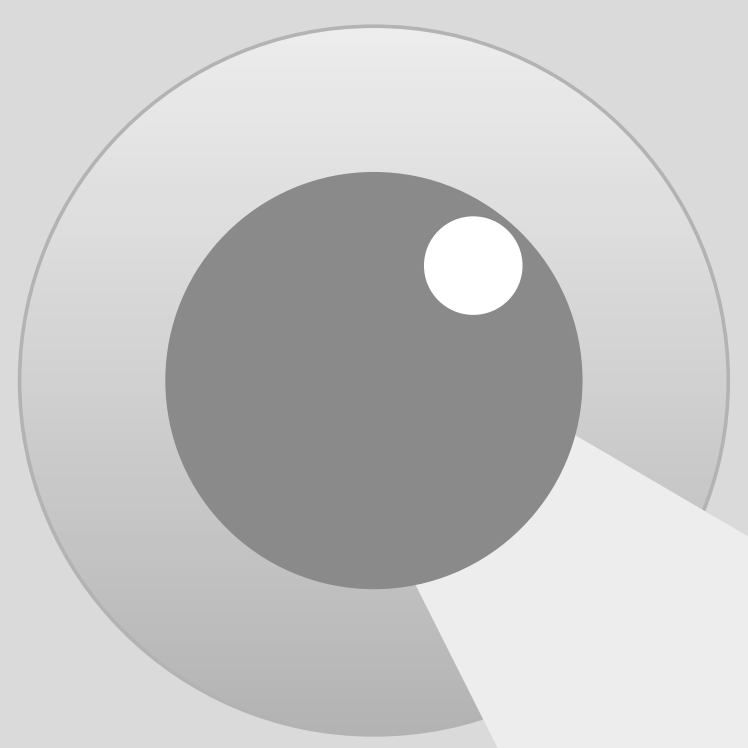


CHAPTER 1 
Diabetes mellitus is a rapidly growing health problem, which affects approximately 382 million people worldwide (International Diabetes Federation, 2013). In the Netherlands, 834,100 people were diagnosed with diabetes on January 1st, 2011 and this number increased by 87,000 patients in that same year (Baan et al., 2014). The prevalence of diabetes in the Netherlands is therefore approaching or already over 1 million (6\% of the total population). The incidence in 2011 concerned 45,000 men and 42,000 women (5.5 per 1.000 men and 4.9 per 1.000 women). In addition to these diagnosed patients, registration data in general practice showed that an estimated $25 \%$ of the patients is as yet undiagnosed in the Netherlands (Rijksinstituut voor Volksgezondheid en Milieu, 2011). Reasons for this high number of undiagnosed patients are amongst others the lack of attention of professionals for early symptoms of diabetes (Baan, 2009). Furthermore, data on the prevalence of diabetes in nursing homes and on the prevalence of diabetes type 2 among children and adolescents appears to be lacking. Of the diagnosed patients, $90 \%$ has type 2 diabetes and the remaining percentage suffers from type 1 diabetes. Type 2 diabetes (formerly called non-insulin-dependent or adult-onset diabetes mellitus) results from the body's ineffective use of insulin. This type has been related to both preventable and non-preventable causes and is largely the result of excess body weight and physical inactivity and until recently it was seen only in adults but it is now also more often occurring in children (World Health Organization, 2013). Type 1 diabetes (previously known as insulin-dependent, juvenile or childhood-onset) is characterized by deficient insulin production and requires daily administration of insulin. The cause of type 1 diabetes is not known and it is not preventable with current knowledge (World Health Organization, 2013).

In the Netherlands, people with diabetes generally perceive a lower quality of life compared to healthy people (without a chronic condition) (Spijkerman et al., 2013). However, people who actually have diabetes but have no complications rate their quality of life only slightly below persons of similar age in the general population (Redekop et al., 2002), and quality of life seems to decrease particularly when complications appear (Redekop et al., 2002).

Diabetes is a complex chronic illness, since it affects various organs and systems and is often accompanied by other diseases, i.e. complications. It requires continuous medical care, ongoing self-management by the patient and support to prevent acute complications, like hyperglycemia or hypoglycemia and reduce the risk of complications in the long run, such as hypertension, cardiovascular diseases or kidney failure (American Diabetes Association, 2013; World Health Organization, 2013). It is estimated that chronic complications occur in 40 to $56 \%$ of all people with diabetes type 2 (Rijksinstituut voor Volksgezondheid en Milieu, 2007). Patients usually do not die of their diabetes but rather from these diabetes-related complications and comorbidities; cardiovascular disease is one the major causes of death among diabetes patients (International Diabetes Federation, 2013). To prevent such complications, enduring control of the disease is needed. Continuity of care is concerned with the quality of health care for patients with 
chronic diseases like diabetes and can be achieved when services are seamlessly linked and this is facilitated by shared management plans between the different health care professionals and the patient or care protocols (Gulliford et al., 2007; Haggerty et al., 2003). Management continuity is particularly important in chronic diseases such as diabetes, since the care for these patients requires optimal coordination and communication between the different health care professionals and organizations that contribute to the patients' care (Gulliford et al., 2007; Haggerty et al., 2003; Reid et al., 2002).

In the Netherlands, attention to continuity of care has increased as a result of the initiative of the Dutch Ministry of Health, Welfare and Sport to start an integrated, programmatic approach of chronic diseases, such as diabetes (Ministerie van Volksgezondheid Welzijn en Sport, 2008). Within this approach the patient is playing a pivotal role and the policy for diabetes is based on four elements: 1) the Netherlands Diabetes Federation (NDF) Care Standard (CS) as basis for the quality of care, 2) the integration of prevention in care, 3) stimulating self-management and 4) stimulating the formation of multidisciplinary teams. The care can be organized in so called chains and purchased through a bundled-payment contract (Ministerie van Volksgezondheid Welzijn en Sport, 2008). The CS and Bundled-payment approach will be explained hereafter.

The care for Dutch diabetes type 2 patients is mainly provided in primary care and delivered by care groups (Spijkerman \& Baan, 2014; Nederlandse Diabetes Federatie, 2013 b). Over the past decade, vertical substitution of care has taken place, i.e. the transfer of tasks between care-providers with different levels of expertise (Vrijhoef et al., 2001). In many general practices the care for diabetes patients is delegated to specialized practice nurses or diabetes nurses who are supervised by the general practitioner. These trained nurses are specialized in care for chronic diseases, such as diabetes and can play an important role in educating patients and encouraging overall adherence to treatment (Ubink-Veltmaat et al., 2005). The care for patients with diabetes type 1 and more complex type 2 patients, for example with multiple complications, is mainly provided in secondary care by internal medicine physicians and diabetes nurses, who are surrounded by a multidisciplinary team of professionals (Nederlandse Diabetes Federatie, 2013a, 2013b).

\section{The Expanded Chronic Care Model}

The concept of continuity of care is reflected in the Expanded Chronic Care Model (Expanded CCM) (figure 1.1), a framework that can be used to optimize the provision of care to patients with chronic diseases (Bodenheimer et al., 2002b), and that advocates integrated care and disease management and the use of evidence-based Care Standards and guidelines (Raad voor de Volksgezondheid en Zorg, 2011). Moreover, the Expanded CCM integrates population based health promotion into the prevention and manage- 
ment of chronic diseases and demonstrates clear associations between the health care system and the community (Barr et al., 2003). According to the model improved health of the population results from positive and productive interactions and relationships among community members, health care professionals, organizations, individuals and community groups. Outcomes include population health outcomes as well as individual functional and clinical outcomes (Barr et al., 2003).

Figure 1.1 shows that the Expanded CCM includes a porous border between the formal health care system (inner oval) and the community, which is a graphical representation of the flow of ideas, resources and people between the health care system and the community. The model identifies four areas of focus which can be integrated within and have an impact on both the health care organization and the community: self-management support, decision support, delivery system and information systems. Self-management support/develop personal skills involves collaboratively helping patients and their family to manage their chronic condition by supporting selfmanagement and developing personal skills for health and wellness. Strategies in the community as well as in the health system are important for this expanded notion of self-management. The delivery system design/re-orient health services involves the health care system to move beyond the provision of clinical services to an expanded mandate supporting individuals and communities in a more holistic, in order to support both health care and population health promotion. Decision support is concerned with the integration of evidence-based standards and guidelines in daily practice and of strategies that facilitate the community's abilities to stay healthy. This support is useful for both dealing with the impact of a disease and making choices that improve health and well-being. Information systems involve computerized information about clinical and functional outcomes as well as population health outcomes important for planning of programs, policies and other initiatives aimed to anticipate on health concerns. Various users will require linked products from information systems and need information about the community beyond the experience of patients within the health care system (Barr et al., 2003).

Furthermore, the Expanded CCM focuses on three elements within the community: Building health public policy, create supportive environments and strengthen community action. Building health policy includes developing and implementing policies to improve population health by ensuring safer and healthier goods, services and environments. The element of Creative supportive environments involves generating safe, stimulating, satisfying and enjoyable living and employment conditions and it includes strategies to stimulate conditions for optimal health in social and community environments. Strengthen community action entails working with community groups to set priorities and achieve goals that enhance the health of the community. Empowerment of the community is important and health care and other professionals can play a role in mobilizing communities to promote health (Barr et al., 2003). 


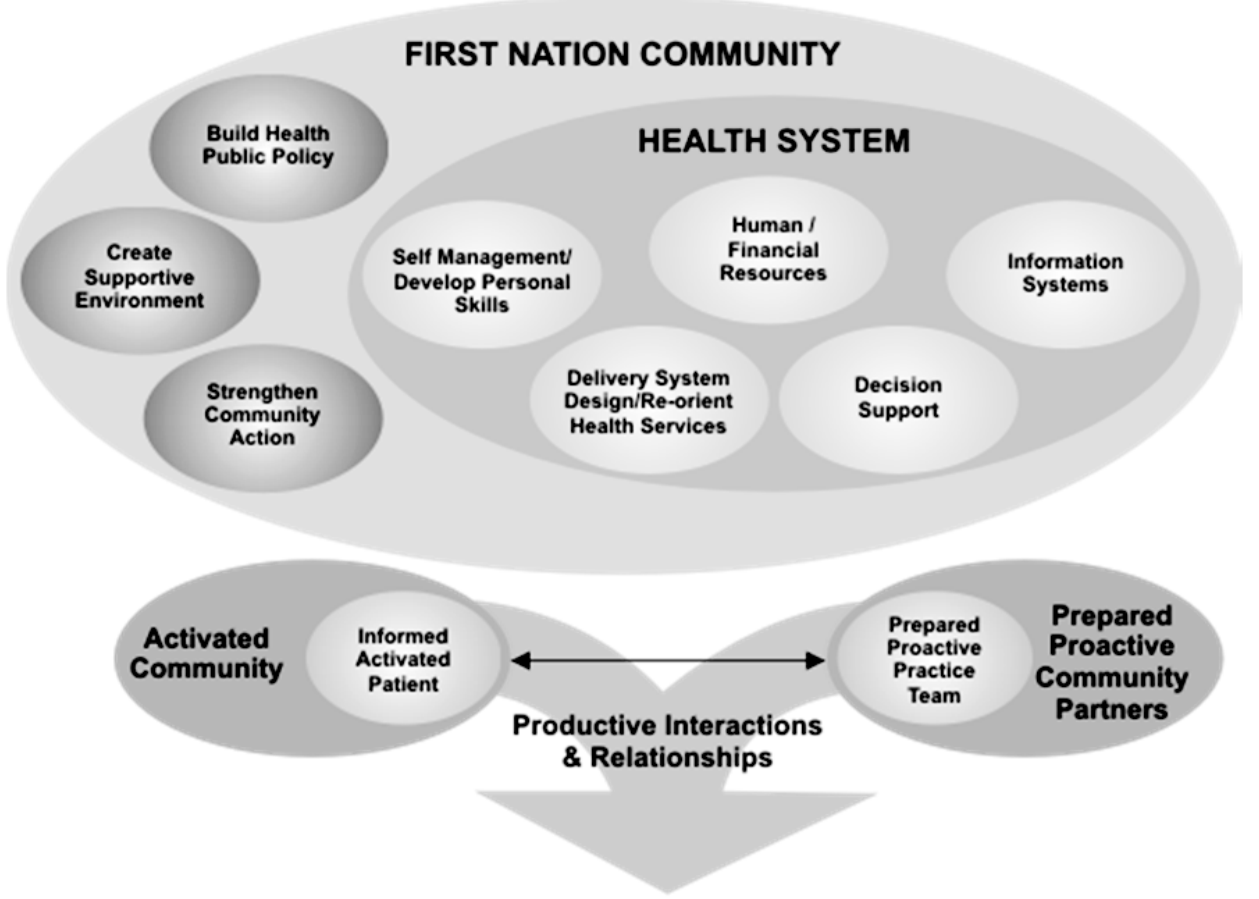

\section{Population Health Outcomes/ Functional \& Clinical Outcomes}

Figure 1.1: The Expanded Chronic Care Model

This dissertation provides a closer look at diabetes care and the introduction of the CS for diabetes from the perspectives of health care professionals and patients. Both will be elaborated below.

\section{Perspective of health care professionals}

\section{National Action program Diabetes and NDF Care Standard}

In Dutch diabetes care, the Expanded CCM is reflected in the National Action program Diabetes (NAD), which was funded by the Ministry of Health, Welfare and Sport (Nederlandse Diabetes Federatie, 2009). The overall purpose of the NAD (2009-2013) was to create the circumstances, conditions and instruments necessary to slow down the increase in the number of people with diabetes and to reduce complications in diabetes patients (Nederlandse Diabetes Federatie, 2009). The main aim of the NAD was the structural implementation of the NDF CS for diabetes. The NAD comprises five subthemes, based on an inventory and analysis of problem areas in the field, and which 
include activities to help achieve the main aim of the NAD: 'Prevention', 'Position of the patient and client', 'Quality, organization and knowledge', 'Rules and funding' and 'Ecommunication and ICT facilities'. For each of these themes, the NAD formulated instrumental objectives, which are implemented in various projects (Nederlandse Diabetes Federatie, 2009). These themes are, accidentally, in line with the concepts of the Expanded CCM (Barr et al., 2003). While the NAD was running, a mixed method design, consisting of a series of quantitative and qualitative studies, was used to monitor the program (Raaijmakers et al., 2010; Raaijmakers, Martens, Hesselink, et al., 2013; Raaijmakers, Hesselink, et al., 2013).

A CS differs from clinical guidelines, in that it is a general framework outlining the services and treatment to be delivered to people with a specific condition on an aggregate level; clinical guidelines on the other hand describe the content of medical care in more detail, including what, why and when care should be provided (Coordinatieplatform Zorgstandaarden, 2010; Nederlandse Diabetes Federatie, 2013d; Seidell et al., 2012). The NDF CS for type 2 diabetes mellitus describes the norm for generic multidisciplinary diabetes care and focuses on the content, organization and quality of diabetes care (Nederlandse Diabetes Federatie, 2013b). The CS is constantly being updated and extended, and is based on evidence-based guidelines. The Dutch Diabetes Association (DVN) and the NDF have together produced a version of the CS (the Zorgwijzer) especially for patients with diabetes, which explains what they can expect from their health care providers (Diabetes Vereniging Nederland). The CS for type 2 diabetes does not apply to type 1 diabetes in adults and children. To fill this gap, a two-part addendum for type 1 diabetes is available, in which the care for adults and for children and adolescents with diabetes type 1 is described (Nederlandse Diabetes Federatie, 2013a). Additionally, indicated prevention was added to the CS by means of an addendum in 2012 (Nederlandse Diabetes Federatie, 2013c).

In the Netherlands, the CS functions as a general overarching framework for the guidelines for each individual professional group and focuses on a multidisciplinary approach to diabetes care. In addition, the CS is used as a purchasing instrument within the Dutch bundled payment approach (Struijs \& Baan, 2011). Health insurers purchase the services and care as described in the CS from a general contractor called the Care Group, which ends up in a so called bundled payment contract. These care groups are relatively new actors in the health care system and are established to improve the quality of chronic care (Inspectie van de Gezondheidszorg, 2012). A care group consists of several health care providers which form a legal unit. Based on the bundled payment contract, the care group assumes financial and clinical accountability and in turn subcontracts individual care providers (like general practitioners, dieticians, internal medicine physicians, etc.) or delivers parts of the services itself for the various components of diabetes care (Struijs \& Baan, 2011).

The Netherlands can be regarded as unique in the use of the CS for diabetes (Seidell et al., 2012). The first Dutch CS for diabetes was published in April 2003 and the NDF 
took a leading role in the implementation of this CS in (Coordinatieplatform Zorgstandaarden, 2010). In the NDF's view, it is necessary to use up-to-date, evidencebased guidelines with multidisciplinary support in the care process, to prevent diabetes, to detect it at an early stage and to provide proper treatment. The introduction of the first CS in 2003 coincided with the development of the 'bundled payment' approach for integrated chronic care (Struijs \& Baan, 2011). This synchronic implementation is not a coincidence since the CS and the bundled payment approach share several principals and concepts and were both included as elements within the integrated, programmatic approach of chronic diseases, as previously described. The bundled payment approach has laid the foundation for delivering and funding diabetes care in accordance with the CS. The CS in turn, has boosted the embedment of the bundled payment approach in Dutch health care. A project to update the CS was started in 2007, and was widely announced in professional journals and at conferences and through contacts with NDF members. The NDF functions as a central contact point within diabetes care for the government and health insurers and unites several stakeholders, including patients, health care professionals (through nine member organizations) and scientists. In 2009, the NDF started the NAD, whose main objective was the systematic nationwide implementation of the CS (Nederlandse Diabetes Federatie, 2009).

The implementation of the CS and Zorgwijzer was supported by several national communication strategies and a regional implementation approach in five pilot regions (Nationaal Actieprogramma Diabetes, 2011). The national strategies that facilitated the implementation of the CS and Zorgwijzer were aimed at knowledge, attitude and behavior of various target groups (i.e. patients, health care professionals and government, health insurers and employers) (Nationaal Actieprogramma Diabetes, 2011). The main objectives were to inform health care professionals and patients about the existence of the CS and its associated benefit, and to make the CS easily accessible online. In addition, the implementation of the CS was facilitated through the development of more than 50 products which were related to the NAD themes and based on existing barriers in practice. Examples of these products include the 'National Transmural Appointment (LTA)' guideline and the 'E-diabetes set'. The NDF selected five pilot regions in the Netherlands to initially implement several of these products with the objective of improving them and then implementing them nationally in the future (Nationaal Actieprogramma Diabetes, 2011).

In the Netherlands, monodisciplinary guidelines for diabetes, such as the clinical practice guidelines issued by the Dutch College of General Practitioners (NHG), have long been available and have been implemented successfully by general practitioners. The need to understand the barriers obstructing optimal health care, the dissemination and implementation of health care innovations and clinical guidelines into daily practice and the extent to which health care professionals actually adhere to guidelines has been emphasized repeatedly (Cochrane et al., 2007; Grol \& Grimshaw, 2003). Previous studies have identified several of these barriers, operating at different levels in the health care 
system (i.e. the level of the patient, the individual professional, the health care team, the health care organization or the wider environment) (Grol et al., 2003; Grol \& Grimshaw, 2003), but not specifically for the use of the CS in the Netherlands. With regard to characteristics of individual professionals, results from a review showed that the main barrier to implementing clinical guidelines is professionals' limited familiarity with or the lack of awareness of particular guidelines (Cabana et al., 1999; Francke et al., 2008). In addition, attitudinal barriers among clinicians, such as physician's considerations of diabetes as a non-serious disease (Dietrich, 1996; Puder \& Keller, 2003) and their lack of knowledge about recent evidence-based guidelines have been suggested to impede faithful and complete implementation (Nam et al., 2010). A review assessing clinicians' attitudes to clinical practice guidelines showed, however, that guidelines are overall seen as helpful resources for advice, valuable educational tools and intended to improve quality (Farquhar, Kofa, et al., 2002). Studies comparing perceptions of guidelines and standards between different groups of health care professionals have been scarce, and the evidence seems inconsistent (Carlsen \& Bringedal, 2011). There are, however, some indications that general practitioners are more reluctant to follow guidelines than professionals working in hospitals and significant differences between general practitioners and other medical doctors have been found in terms of their attitudes toward clinical guidelines (Carlsen \& Bringedal, 2011). As regards environmental factors, limited time, staff shortages, work pressure and financial disincentives have been found to be the main barriers to guideline implementation (Cabana et al., 1999; Francke et al., 2008; Grol \& Grimshaw, 2003).

From the perspective of health care professionals, this dissertation aims to answer the following research questions:

1. How are the Care Standard for diabetes and the National Action program Diabetes implemented in practice?

2. What are health care professionals' perceptions regarding the Care Standard for diabetes?

3. What facilitators and barriers do health care professionals perceive in diabetes care and in relation to the implementation of the Care Standard?

\section{Perspective of diabetes patients}

\section{Self-management and motivation}

Many aspects of the care for diabetes patients are nowadays managed by patients themselves on a life-long basis (Barlow et al., 2002; Reed et al., 2003). As a result, the responsibility for day-to-day disease management is shifting from health care professionals to the individuals, which places a considerable burden on these patients (Barlow 
et al., 2002; Reed et al., 2003). Self-management refers to the individual's ability to manage the symptoms, treatment, physical and psychosocial consequences and life style changes inherent in living with a chronic condition (Barlow, Wright et al. 2002). The idea of self-management is based on the premise that people have the capacity to make choices and are responsible for the consequences of their choices (Feste \& Anderson, 1995). It is not the health care professional but the patient that needs to be in control in order to perform adequate self-management on a daily basis. Diabetes self-care involves a range of activities such as self-monitoring of blood glucose, eating a healthy diet, being physically active, taking the recommended medication and consulting health care professionals (Toobert, 2000). Dutch (EADV \& NAD, 2012) and international recommendations (American Diabetes Association, 2013) advise daily self-monitoring of blood glucose for patients with type 1 diabetes and for patients with type 2 diabetes who use insulin therapy. In contrast to some general assumptions that adherence to self-care activities leads to an increase in perceived health and quality of life, some previous studies have also indicated that engaging in self-management behaviors has only a minor effect on perceived health, quality of life and diabetes control (Maddigan et al., 2005; Watkins et al., 2000). Since there is no evidence that self-control in type 2 patients without insulin treatment is beneficial on clinical outcomes, daily self-monitoring in these patients can particularly be useful in special circumstances, e.g. when patients are pregnant or have disordered blood glucose levels (EADV \& NAD, 2012). Ideally, health care professionals aim to promote optimal self-management among their diabetes patients next to their own responsibilities concerning periodical physical examinations and checks on biomedical parameters, medication adjustments and provision of advice and support. Previous studies have shown that better adherence to self-management of diabetes patients can improve their glycemic control (Gao et al., 2013; Heisler et al., 2003; Norris et al., 2001) and quality of life (Cochran \& Conn, 2008). However, other studies have indicated that engaging in self-management behaviors only has a minor effect on perceived health, quality of life and diabetes control (Maddigan et al., 2005; Watkins et al., 2000).

Patients are now playing a more pivotal role in the treatment of their disease due to self-management and they are expected to continuously monitor their diabetes. Motivation is needed to enable patients to self-manage their disease. It will not be easy for patients to consciously consider every activity they have to undertake. Instead, they are (preferably intrinsically) motivated and their actions become automatic responses.

\section{Self-determination theory}

\section{Intrinsic versus extrinsic motivation}

The Self Determination Theory (SDT) provides a broad framework for the study of human motivation and personality. SDT focuses on the degree to which an individual's 
behavior is self-motivated and self-determined. SDT distinguishes between intrinsic and extrinsic motivation (Deci \& Ryan, 1985). Intrinsic motivation refers to doing something because it is inherently interesting or enjoyable and is per definition self-determined (autonomous). Extrinsic motivation, in contrast, refers to doing something because it leads to a separable outcome (which has instrumental value) and is often more controlled (i.e. less autonomous) (Ryan \& Deci, 2000). SDT distinguishes four types of extrinsic motivation based on the degree to which the motivation and regulation of this extrinsic motivation have been internalized (i.e. integrated in the person). These types are, in order from the least to the most fully internalized: external regulation (i.e. selfmonitoring blood glucose because the doctor wants it), introjected regulation (i.e. selfmonitoring blood glucose because otherwise you will feel guilty), identified regulation (i.e. self-monitoring blood glucose because it makes the patient proud on itself), and integrated regulation (i.e. self-monitoring blood glucose because it makes you healthier) (Ryan \& Deci, 2000).

Furthermore, SDT states that people are more likely to intrinsically adopt healthy behaviors or self-care activities when their basic psychological needs for autonomy, competence and relatedness are supported (Ryan \& Deci, 2000). When satisfied, these needs lead to enhanced self-motivation, while they can lead to diminished motivation and well-being when they are not satisfied (Ryan \& Deci, 2000).

\section{Treatment self-regulation and perceived competence}

Within SDT, the concepts of treatment self-regulation and perceived competence are assumed to be important in initiating and maintaining diabetes self-care behaviors. Treatment self-regulation is concerned with the degree to which a person's motivation to engage in healthy behavior or follow a treatment regimen is relatively autonomous versus controlled (Williams et al., 2009). Patients who take their medication because they believe in its efficacy and are personally committed to improving their health are considered autonomous, whereas their behavior is considered to be controlled if they take their medication because their health care providers or social environment pressures them to do so (Williams, Freedman, et al., 1998). Perceived competence means that patients are capable and effective in achieving desired outcomes (Williams et al., 2009). Previous research has shown that people are more likely to feel competent to achieve important outcomes when they feel autonomous to make behavioral choices (Williams, Freedman, et al., 1998).

Previous studies have shown that increases in perceived autonomy and competence are related to improved and blood glucose testing (Shigaki et al., 2010) and glycemic control (Williams, Freedman, et al., 1998; Williams et al., 2004). Moreover, only autonomous motivations have been found to yield the long-term persistence and adherence that are needed for patients with diabetes to keep their glucose within a healthier range (Williams, Freedman, et al., 1998). Previous studies have also reported a positive association between autonomous motivation and success in maintaining a broad range of 
health-related behaviors (Williams, Gagné, et al., 2002; Williams, Gagne, et al., 2002; Williams et al., 1996).

\section{Perceived autonomy support}

The quality of social contexts influences the motivation, performance and well-being of individuals who operate within them. In SDT, the concept of autonomy support is used to characterize the quality of the health care environment. Autonomy support can be seen as an orientation towards patient-centered care among health care providers. Autonomy support facilitates patients' autonomous self-regulation and perceived competence for healthy behaviors (Williams et al., 2009). Previous studies have shown that patients report enhanced autonomy and competence in diabetes self-management activities when they perceive their doctors to be more autonomy-supportive (Ryan \& Deci, 2000; Williams, Freedman, et al., 1998; Williams et al., 2004; Williams et al., 2009). Furthermore, clinicians' autonomy support has been found to correlate with psychosocial, physiological and biological outcomes regarding diabetes self-management (Williams et al., 2005) and quality of life (Williams et al., 2009). The current empirical base regarding the association of SDT concepts with diabetes outcomes is mostly built on studies from the United States.

\section{General causality orientations and mastery}

SDT also proposes that people have dispositional tendencies, named causality orientations which are general motivational orientations that refer to (a) the way people orient to the environment concerning information related to the initiation and regulation of behavior, and thus (b) the extent to which they are self-determined in general, across situations and domains (Deci \& Ryan, 2008). According to the more general causality orientations perspective, it is possible to assess an individual's tendency to orient to and be guided by each of three general sources of behavioral regulation: autonomy-, controland impersonal orientation. The autonomy orientation involves a high degree of experienced choice with respect to the initiation and regulation of people's own behavior (Deci \& Ryan, 1985). Autonomy oriented people look for opportunities for self-determination and choice and are having a generalized tendency toward an internal perceived locus of causality. These people are more often intrinsically motivated and more likely to be selfdetermined with respect to extrinsic rewards (Deci \& Ryan, 1985). Within the control orientation people's behavior is organized with respect to controls either in the environment or inside themselves. When people are control oriented, extrinsic rewards play a more important role in their behavior (Deci \& Ryan, 1985). The impersonal orientation involves people experiencing their behavior as being beyond their intentional control. They tend to believe they are unable to regulate their behavior in a way that will lead reliably to desired outcomes. When high on the impersonal orientation, people see themselves as incompetent and unable to master situations (Deci \& Ryan, 1985). 
Closely and inversely related to the impersonal orientation of behavioral regulation is the concept of personal mastery, which consists of a general sense of control over one's life and circumstances (Pearlin \& Schooler, 1978). A high level of mastery has been found to be associated with better health in people with chronic conditions or disability (Cott et al., 1999). Few previous studies have examined personal mastery in relation to psychological, behavioral or physical outcomes among diabetes patients. These studies showed that diabetes patients who reported a higher level of mastery, had better scores on physical, mental and social functioning (Mertens et al., 2012), engaged in more recommended care practices and had higher scores on perceived health (Sloan et al., 2009). The concept of locus of control, which is based on Social Learning Theory, is closely linked to mastery and has been described as 'the amount of personal control over the environment individuals believe to that they possess' (Rotter, 1966). Previous studies reported no significant association between locus of control and self-management in diabetes patients (Coates \& Boore, 1998; Mansour-Ghanaei et al., 2013).

From the perspective of patients, this dissertation aims to answer the following research questions:

1. How are perceived autonomy support, perceived competence, treatment selfregulation and mastery associated with patient's self-management, general diabetes control and health-related quality of life?

2. Are there differences in mastery, perceived autonomy support, perceived competence, self-management and health-related quality of life between subgroups of patients (in terms of age, gender, educational level, BMI and co-morbidities)?

\section{Aims and outline of this dissertation}

The aim of this dissertation was to provide a closer look at diabetes care and the introduction of the Netherlands Diabetes Federation (NDF) Care Standard (CS) for diabetes from the perspectives of both health care professionals and diabetes patients. From the perspective of health care professionals, this dissertation assessed their perceptions regarding the content and implementation of the CS and perceived facilitating and impeding factors in diabetes care. From the perspective of patients, this dissertation examined how Self-Determination Theory concepts and mastery were associated with Dutch type 1 and type 2 diabetes patients' self-management activities, general diabetes control and health-related quality of life.

In Chapter 2 a cross-sectional questionnaire study assessing perceptions of Dutch health care professionals regarding the CS and the perceived barriers to use the CS, is described in order to suggest ways to optimize the implementation of the CS.

Chapter 3 reports on a study that examined perceived facilitating and impeding factors among health care professionals in diabetes care, using a qualitative research 
design. The results are based on 18 semi-structured interviews with health care professionals from all professions relevant to diabetes care. The CCM was used to classify the facilitators and barriers into key elements of the health care system.

Chapter 4 describes a cross-sectional questionnaire study examining how Dutch type 1 and type 2 diabetes patients' perceived autonomy support from their primary care provider, as well as their perceived competence and treatment self-regulation, are associated with their diabetes self-care activities (healthy diet, physical activity, monitoring blood glucose and medication use) and general diabetes control.

In Chapter 5 a cross-sectional questionnaire survey which assessed the associations between type 2 diabetes patients' mastery and perceived autonomy support and their self-management skills and health-related quality of life, is described.

Chapter 6 reports on two cross-sectional questionnaire studies conducted in 2010 and 2013 among health care professionals and type 1 and type 2 diabetes patients. The main aim of these studies was to assess the implementation of the CS for diabetes and compare the results of both studies.

In chapter 7 a perspective on the implementation of the National Action program Diabetes in the past decade is provided, which is based on a series of quantitative and qualitative studies. This perspective may function as an evaluation moment and example for similar approaches in other countries.

The dissertation concludes with a general discussion in chapter 8 , in which the main findings from the preceding chapters and methodological considerations are discussed as well as implications for practice, theory and further research. 


\section{Chapter 2}

\section{Perceptions of Dutch health care professionals regarding the Care Standard for diabetes}

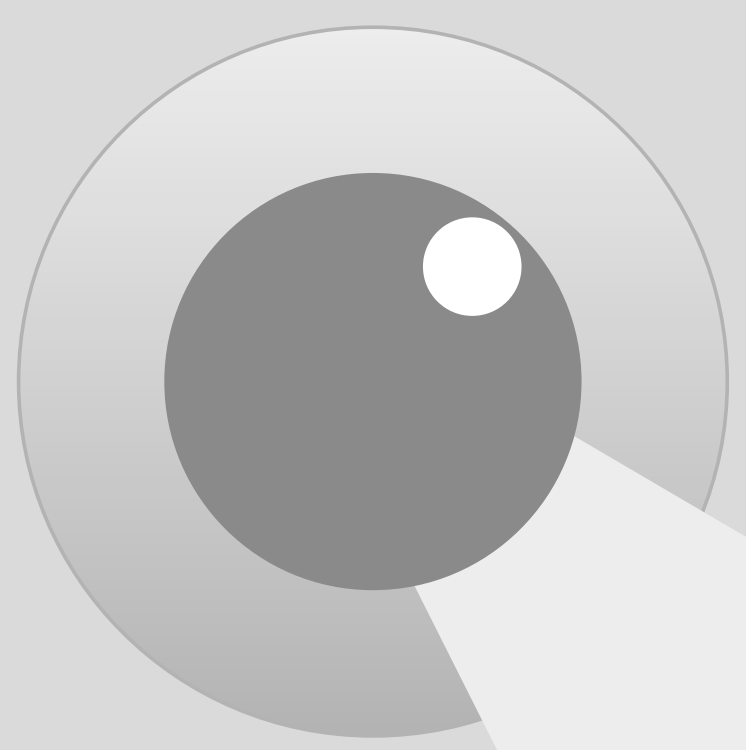

Published as: Raaijmakers, L.G.M., Martens, M.K., Bagchus, C., de Vries, N.K., Kremers, S.P.J. (2013). Perceptions of Dutch health care professionals regarding the Care Standard for diabetes. BMC Research Notes, 17(6), 417. 


\section{Abstract}

Background: The Netherlands can be regarded as unique in the use of the Netherlands Diabetes Federation (NDF) Care Standard (CS) for diabetes. The need to understand the barriers obstructing optimal health care, the dissemination and implementation of health care innovations into daily practice and the extent to which health care professionals actually adhere to guidelines has been emphasized repeatedly. Therefore, the aim of the present study was to suggest ways to optimize the implementation of the CS by examining the perceptions of Dutch health care professionals regarding the CS and the barriers to using it.

Methods: A cross-sectional questionnaire survey was conducted among health care professionals ( $\mathrm{N}=1547)$ in 2010 .

Results: A total of $39.6 \%(\mathrm{~N}=1323)$ of the participating health care professionals possessed the CS. Only $15.5 \%$ of the professionals who were to some extent familiar with the CS $(N=1100)$ described themselves as working in complete accordance with the CS. The majority (83.9\%) thought the CS contributed greatly to ensuring the quality of care; the judgment on the feasibility of working in accordance with the CS was positive (mean $=3.9$ on a 5 -point Likert scale). However, professionals tended to perceive the guidelines issued by the own professional association as the norm for high quality diabetes care, rather than the CS. The main barrier to using the CS was the lack of effective lifestyle interventions (or access to them) to provide care for people with diabetes or those at increased risk for the disorder.

Conclusions: A limited percentage of health care professionals were found to possess the CS. It is questionable whether possession of the CS is a prerequisite for delivering high quality care. Overall, professionals were largely positive about the CS, although only a minority indicated they were working in complete accordance with it. Professionals and professional organizations should be further educated about the content of the CS and especially its added value with respect to the guidelines for their own professional group, in terms of the multidisciplinary approach to diabetes care. Furthermore, attention should be given to the most important perceived barriers, to facilitate adherence to the CS. 


\section{Background}

Diabetes mellitus is a rapidly growing health problem, which affects approximately 371 million people worldwide (International Diabetes Federation, 2012). The prevalence of diabetes in the Netherlands is approaching 1 million (6\% of the total population) and this number is increasing by 87,000 patients each year (Baan et al., 2014). Diabetes is a complex chronic illness, since it affects various organs and systems and is often accompanied by other diseases. Hence, diabetes requires continuous medical care and ongoing patient self-management and support to prevent acute complications and reduce the risk of complications in the long run (American Diabetes Association, 2013). Continuity of care is concerned with the quality of health care for patients with chronic conditions like diabetes and can be achieved when services are seamlessly linked and this is facilitated by shared management plans or care protocols (Gulliford et al., 2007; Haggerty et al., 2003).

In the Netherlands, attention to continuity of care has increased as a result of the initiative of the Dutch Ministry of Health, Welfare and Sport to start an integrated, programmatic approach of chronic diseases (Ministerie van Volksgezondheid Welzijn en Sport, 2008). The concept of continuity of care is also reflected in the Chronic Care Model (CCM), a framework that can be used to optimize the provision of care to patients with chronic conditions (Bodenheimer et al., 2002b), and that advocates integrated care and disease management and the use of evidence-based Care Standards and guidelines (Raad voor de Volksgezondheid en Zorg, 2011). The CCM focuses on improving and optimizing six key elements of the health care system: community resources and policies, organization of health care, self-management support, delivery system design, decision support and clinical information systems (Bodenheimer et al., 2002b). In Dutch diabetes care, the CCM is reflected in the National Diabetes Action Program (NAD), which is funded by the Ministry of Health, Welfare and Sport (Nederlandse Diabetes Federatie, 2009). The overall purpose of the National Diabetes Action Program (2009-2013) is to create the circumstances, conditions and instruments necessary to slow down the increase in the number of people with diabetes and to reduce complications in diabetes patients (Nederlandse Diabetes Federatie, 2009). The main objective of the action program is the systematic implementation of the Netherlands Diabetes Federation (NDF) Care Standard (CS) for the content, organization, quality and funding of diabetes prevention and care (Nederlandse Diabetes Federatie, 2009). A Care Standard is a general framework outlining the services and treatment of people with a specific condition on a very aggregated level, while clinical guidelines describe the content of care in more detail, including how, when and by whom care should be provided (Coordinatieplatform Zorgstandaarden, 2010; Seidell et al., 2012). The NDF CS for type 2 diabetes mellitus describes the norm for generic multidisciplinary diabetes care and focuses on the content, organization and quality of diabetes care. The CS is constantly being updated and extended, and is based on evidence-based guidelines. The CS functions as a general overarching framework for 
the guidelines for the individual professional groups and focuses on a multidisciplinary approach to diabetes care. In addition, the CS is used as a purchasing instrument within the Dutch bundled payment approach. In the Netherlands, insurers purchase the services and care as described in the CS from a general contractor (called the Care Group), which ends up in a so called bundled payment contract. Based on this contract, the Care Group assumes financial and clinical accountability and in turn subcontracts individual care providers (like the GP, dietician, internal medicine physicians, etc.) or delivers parts of the services by itself (Struijs \& Baan, 2011).

The Netherlands can be regarded as unique in the use of the CS for diabetes (Seidell et al., 2012). The first Dutch CS for diabetes was published in April 2003 at the request of, and with financial support by the Ministry of Health, Welfare and Sport, in collaboration with the ZN health care insurers association (Coordinatieplatform Zorgstandaarden, 2010). The NDF has adopted a leading role in the implementation of the CS in the Netherlands. In the NDF's view, it is necessary to use up-to-date, evidence-based guidelines with multidisciplinary support in the care process, to prevent diabetes, to detect it at an early stage and to provide proper treatment. The introduction of the first CS in 2003 coincided with the development of the 'bundled payment' approach for integrated chronic care (Struijs \& Baan, 2011). This approach has laid the foundation for delivering and funding diabetes care in accordance with the CS. A project to update the CS was started in 2007, and was announced by the NDF through professional journals and conferences and through their contacts with the NDF members. In 2009, the NDF started the NAD, whose main objective was the systematic nationwide implementation of the Care Standard (Nederlandse Diabetes Federatie, 2009).

In the Netherlands, monodisciplinary guidelines for diabetes, such as the clinical practice guidelines issued by the Dutch College of General Practitioners (NHG), have long been available and have been implemented successfully by general practitioners. The need to understand the barriers obstructing optimal health care, the dissemination and implementation of health care innovations into daily practice and the extent to which health care professionals actually adhere to guidelines has been emphasized repeatedly (Cochrane et al., 2007; Grol \& Grimshaw, 2003). Previous studies have identified several of these barriers, operating at different levels in the health care system (i.e. the level of the patient, the individual professional, the health care team, the health care organization or the wider environment) (Grol et al., 2003; Grol \& Grimshaw, 2003), but not specifically for the use of the CS in the Netherlands. With regard to characteristics of individual professionals, results from a review showed that the main barrier to implementing clinical guidelines is professionals' limited familiarity with or the lack of awareness of particular guidelines (Cabana et al., 1999; Francke et al., 2008). In addition, attitudinal barriers among clinicians and their lack of knowledge about recent evidence-based guidelines have been suggested to impede faithful and complete implementation (Farquhar, Kofa, et al., 2002; Nam et al., 2010). A study of clinicians' attitudes showed, however, that guidelines are seen as helpful resources for advice and as valuable educa- 
tional tools that might improve quality (Farquhar, Kofa, et al., 2002). Studies comparing perceptions of guidelines and standards between different groups of health care professionals have been scarce, and the evidence seems inconsistent (Carlsen \& Bringedal, 2011). There are, however, some indications that general practitioners are more reluctant to follow guidelines than professionals working in hospitals (Carlsen \& Bringedal, 2011). Moreover, a recent study in Norway found significant differences between general practitioners and other medical doctors in terms of their attitudes toward clinical guidelines (Carlsen \& Bringedal, 2011). As regards environmental factors, limited time, staff shortages and pressure of work have been found to be the main barriers to guideline implementation (Cabana et al., 1999; Francke et al., 2008).

The aim of the present study was to suggest ways to optimize the implementation of the CS by examining the perceptions of Dutch health care professionals regarding the CS and the barriers to using it. We decided to specifically assess the problem from the perspective of the health care professionals, since this provides us with insights into the perceptions of all involved disciplines that have to work according to the same principles. The specific research questions addressed in this study were: (1) To what extent are health care professionals familiar with the CS and do they perceive themselves as working in accordance with the CS? (2) How do health care professionals appreciate the CS? (3) Are their differences in adherence to and the appreciation of the CS between subgroups (in terms of age, gender, profession and health care division)? (4) Which barriers do health care professionals perceive in working in accordance with the CS?

\section{Methods}

\section{Design and procedure}

A cross-sectional questionnaire survey was conducted among health care professionals ( $N=1547$ ) between June and November 2010. The most important strategy used to recruit participants, involved a mailing of personalized letters with a link to a web-based questionnaire for all professions involved in diabetes care: general practitioners $(\mathrm{N}=$ $443)$, practice nurses $(N=441)$, diabetes nurses $(N=224)$, dieticians $(N=163)$, physiotherapists $(N=142)$, internal medicine physicians $(N=68)$ and pediatricians $(N=66)$. The addresses were acquired either from the databases of the professional associations of the relevant professions (e.g. the Dutch Dietetic Association) or by purchasing randomly selected commercially available addresses of health care providers'. In addition, an invitation to participate was placed on the websites and/or in the newsletter of several professional associations of health care providers (e.g. the Dutch College of General Practitioners). Ethical approval was not required for this study. 


\section{Measures}

General characteristics that were assessed included gender, age and the health care division the professional was working in (i.e. primary or secondary care). Familiarity with the CS was measured with one item asking participants whether they were familiar with the CS. Answer options were: I possess the CS; I have seen it, but do not possess it; I have heard about it, but do not possess it; and I am unfamiliar with it. This variable was recorded into a dichotomous 'possession of CS' variable ('does not possess' (1) - 'possesses it' (2)). Professionals' perception of their own adherence to the CS was assessed with one item, asking participants to what extent they perceived themselves as working in accordance with the CS ( 1 = not at all; to $5=$ completely). This variable was recorded into a dichotomous variable ('working not at all or only partly in accordance with the CS' (1) - 'working in complete accordance with the CS' (2)). Appreciation of the CS was measured with three items. Two items, scored on 5-point Likert scales ( 1 = not at all; to 5 = completely), assessed whether professionals regarded the CS as the norm for high quality diabetes care and whether they regarded the guidelines issued by the own professional association as the norm for high quality diabetes care. A third item asked whether they thought the CS contributed to ensuring quality of care ('no contribution' (1) - 'major contribution' (3)). This variable was recorded into a dichotomous 'quality assurance' variable ('no or only minor contribution' (1) - 'major contribution' (2)) and analyzed separately. The feasibility of working in accordance with the CS was assessed with one item asking whether participants thought it was feasible to work in accordance with the CS ( 1 = not at all; to 5 = completely). Barriers were measured by asking participants to indicate, on a list of items, which one(s) they experienced as barriers. Examples of barriers included 'lack of effective lifestyle interventions (or access to them) to provide care for people with diabetes or at increased risk of diabetes', 'financial, legislative and regulations issues impeding care and prevention in accordance with the CS' and 'collaboration between primary and secondary care'. Multiple answers were allowed and an open answer category was provided to add further perceived barriers.

The questionnaire was developed in close collaboration with advisors of the Dutch Diabetes Federation and health promotion research experts and pretested among at least two professionals of each professional group involved in our study.

\section{Statistical analyses}

Data were analyzed using SPSS 17.0. Descriptive statistics and frequencies were used to analyze the respondents' familiarity with and appreciation of the CS. Associates of working in accordance with the CS and CS quality assurance were determined using logistic regression analyses, via the ENTER method, with age, gender, profession and health care division as independent variables. Similar linear regression analyses were conducted to determine associates of appreciation of the CS and the feasibility of working in accordance with the CS. Contrasts between associates of the independent variables mentioned 
above were tested by repeating all regression analyses using a different reference group for each categorical variable each time. P-values $<0.05$ were considered to be statistically significant.

\section{Results}

The mean age of the professionals was 46.9 (SD 8.6) years and the majority were female (66.3\%). Of the professionals, $77.3 \%$ were working in primary care and $22.7 \%$ were working in secondary care.

\section{Familiarity with and adherence to the Care Standard}

Of the health care professionals who answered the question on familiarity with the CS for diabetes ( $N=1323$ ), 39.6\% possessed the standard, while $19.7 \%$ had seen the CS, but did not possess it; $23.8 \%$ had heard about it, but did not possess it and $16.9 \%$ were unfamiliar with it. This last group was excluded from further analyses. Table 2.1 shows the familiarity with the CS for each of the professional groups. Diabetes nurses were most likely to possess the CS, followed by dieticians and internal medicine physicians, whilst physiotherapists were the least likely to possess the CS. Of the health care professionals who were to some extent familiar with the CS ( $N=1100), 15.5 \%$ (which is $11.0 \%$ of all respondents) perceived themselves as working in complete accordance with the CS.

Table 2.1: Possession of the Care Standard by professional group

\begin{tabular}{lccc}
\hline Health care professionals & $\mathrm{N}$ & \multicolumn{2}{c}{ Familiarity with Care Standard (\%) } \\
\cline { 3 - 4 } & & In possession & Not in possession \\
\hline General Practitioner & 431 & 40.4 & 59.6 \\
Practice nurse & 376 & 35.4 & 64.6 \\
Diabetes nurse & 187 & 55.1 & 44.9 \\
Dietician & 124 & 48.4 & 51.5 \\
Physiotherapist & 79 & 15.2 & 84.8 \\
Internal medicine physician & 65 & 41.5 & 58.5 \\
Pediatrician & 61 & 24.6 & 75.4 \\
\hline
\end{tabular}

\section{Appreciation of the Care Standard}

Table 2.2 presents the appreciation of the CS among the professionals who were familiar with it. The respondents tended to perceive the guidelines issued by the own professional association as the norm for high quality diabetes care, rather than the CS. Diabetes nurses and dieticians were the least positive about the CS being the norm for high quality diabetes care, even though a relatively large part of the former group (55.4\%) 
possessed the CS. Nevertheless, the majority of the respondents thought the CS made a major contribution to ensuring the quality of care. Working in accordance with the CS was mostly judged to be feasible.

\section{Associates of adherence to and appreciation of the CS}

Table 2.2 also provides an overview of differences between subgroups in terms of working in accordance with the CS and appreciation of the CS. It shows that the younger respondents were less likely to perceive themselves as working in complete accordance with the CS. Female respondents were less positive about the CS being the norm for high quality diabetes care. Respondents working in secondary care were less likely to perceive themselves to be working in complete accordance with the CS and were less positive about the CS being the norm than respondents working in primary care. Dieticians and physiotherapists were less likely to work in complete accordance with the CS than general practitioners, practice nurses, diabetes nurses, internal medicine physicians and pediatricians. Practice nurses, diabetes nurses, dieticians and physiotherapists were less positive about the CS being the norm than general practitioners, internal medicine physicians and pediatricians. By contrast, physiotherapists were less positive about the guidelines issued by the own professional being the norm than general practitioners, practice nurses, diabetes nurses, dieticians and pediatricians. Compared to general practitioners, all other participating professional groups gave a lower score for the feasibility of using the CS. Finally, practice nurses and diabetes nurses were significantly more positive about the contribution of the CS to ensuring quality of care than the other professional groups.

\section{Perceived barriers}

A total of $68.4 \%$ of the respondents reported perceiving barriers to diabetes care; the mean number of reported barriers was 3.1 (SD 2.1, range 1-16). Table 2.3 presents the three most frequently mentioned perceived barriers, both in general and for each participating professional group separately. In general, the most important barrier was the lack of effective lifestyle interventions (or access to them) to provide care for people with diabetes (or at increased risk for diabetes); this was also the dominant barrier reported by the general practitioners, practice nurses and internal medicine physicians. The diabetes nurses and dieticians perceived the care for groups who are difficult to reach, such as ethnic minorities and people with a low SEP, as the most important barrier. The physiotherapists reported the financial, legislative and regulations issues as the most important barrier to providing care and prevention in accordance with the CS. Pediatricians reported the lack of refresher courses about counseling for selfmanagement as the most important barrier. All respondents, except the internal medi- 
cine physicians, listed the financial, legislative and regulations issues among their top five barriers.

Table 2.2: Associates of heath care professionals' appreciation of and adherence to the Care Standard $(\mathrm{N}=$ 1081)

\begin{tabular}{|c|c|c|c|c|c|}
\hline & $\begin{array}{l}\text { Working in } \\
\text { accordance } \\
\text { with CS (\%) } \\
\text { Completely }\end{array}$ & $\begin{array}{l}\text { CS as norm } \\
\text { (mean(SD)) }\end{array}$ & $\begin{array}{l}\text { Guidelines for } \\
\text { professional } \\
\text { group as norm } \\
\text { (mean(SD)) }\end{array}$ & $\begin{array}{l}\text { Feasibility } \\
(\text { mean(SD)) }\end{array}$ & $\begin{array}{l}\text { Quality assurance } \\
\qquad(\%)\end{array}$ \\
\hline All participants & 15.5 & $2.9(1.0)$ & $4.0(0.4)$ & $3.9(0.7)$ & 83.9 \\
\hline Age & $\mathrm{OR}=1.03^{*}$ & $\beta=-0.02$ & $\beta=-0.36$ & $\beta=0.05$ & $O R=1.00(0.98-1.02)$ \\
\hline \multicolumn{6}{|l|}{ Gender } \\
\hline Men (1) & 18.0 & $3.3(1.0)$ & $4.0(0.3)$ & $4.0(0.7)$ & 83.0 \\
\hline Women (2) & 14.1 & $2.7(1.0)$ & $4.0(0.5)$ & $3.9(0.7)$ & 84.3 \\
\hline Significant contrasts & & $2<1$ & & & \\
\hline \multicolumn{6}{|l|}{ Professional group } \\
\hline General Practitioners (1) & 27.8 & $3.0(1.0)$ & $4.0(0.2)$ & $4.1(0.7)$ & 82.1 \\
\hline Practice nurses (2) & 12.7 & $2.7(0.7)$ & $4.0(0.3)$ & $3.9(0.5)$ & 90.0 \\
\hline Diabetes nurse s(3) & 15.1 & $2.3(0.9)$ & $4.1(0.4)$ & $3.8(0.7)$ & 89.7 \\
\hline Dieticians (4) & 3.4 & $2.1(0.7)$ & $4.0(0.7)$ & $3.6(0.7)$ & 78.6 \\
\hline Physiotherapists (5) & 1.4 & $2.7(0.9)$ & $3.8(0.9)$ & $3.5(0.9)$ & 75.7 \\
\hline Internal medicine & 7.8 & $3.2(1.2)$ & $3.8(0.8)$ & $3.7(0.9)$ & 64.0 \\
\hline physicians (6) & 19.6 & $3.4(1.1)$ & $4.0(0.0)$ & $3.7(0.9)$ & 73.9 \\
\hline \multicolumn{6}{|l|}{ Pediatricians (7) } \\
\hline Significant contrasts & $2<1,7$ & $2,3,4,5<1,6,7$ & $5<1,2,3,4,7$ & $2,3,4,5,6,7<1$ & $6<1,4$ \\
\hline & $4,5<1,2,3,7$ & $3,4<2,5$ & $6<3$ & $4,5<2,3$ & $1,4,5,6,7<2,3$ \\
\hline & $5<6$ & & & & $5<1$ \\
\hline \multicolumn{6}{|l|}{ Health care division } \\
\hline Primary care (1) & 17.2 & $3.0(1.0)$ & $4.0(0.4)$ & $4.0(0.7)$ & 85.4 \\
\hline Secondary care (2) & 9.3 & $2.5(1.1)$ & $4.0(0.6)$ & $3.7(0.8)$ & 78.9 \\
\hline Significant contrasts & $2<1$ & $2<1$ & & & \\
\hline
\end{tabular}

$* p<0.05$; Note: significant contrasts were identified by comparisons made between the subgroups by repeating the logistic or linear regression analysis using a different reference group for each independent variable each time 
Table 2.3: Top 3 barriers perceived, by professional group $(N=687)$

\begin{tabular}{|c|c|c|}
\hline Health care professionals & Perceived barriers & $\%$ \\
\hline \multirow[t]{3}{*}{ All participants } & $\begin{array}{l}\text { Lack of effective lifestyle interventions (or access to them) to provide } \\
\text { care for people with diabetes (or at increased risk for it) }\end{array}$ & 41.0 \\
\hline & $\begin{array}{l}\text { Care for groups that are difficult to reach, such as ethnic minorities } \\
\text { and people with a low SEP }\end{array}$ & 36.3 \\
\hline & $\begin{array}{l}\text { Financial, legislative and regulations issues regarding care and preven- } \\
\text { tion in accordance with the Care Standard }\end{array}$ & 31.1 \\
\hline \multirow[t]{3}{*}{ General practitioners } & $\begin{array}{l}\text { Lack of effective lifestyle interventions (or access to them) to provide } \\
\text { care for people with diabetes (or at increased risk for it) }\end{array}$ & 55.4 \\
\hline & $\begin{array}{l}\text { Care for groups that are difficult to reach, such as ethnic minorities } \\
\text { and people with a low SEP }\end{array}$ & 27.5 \\
\hline & Lack of refresher courses about lifestyle counseling & 24.6 \\
\hline \multirow[t]{3}{*}{ Practice nurses } & $\begin{array}{l}\text { Lack of effective lifestyle interventions (or access to them to provide } \\
\text { care for people with diabetes (or at increased risk for it) }\end{array}$ & 39.6 \\
\hline & $\begin{array}{l}\text { Financial, legislative and regulations issues regarding care and preven- } \\
\text { tion in accordance with the Care Standard }\end{array}$ & 30.0 \\
\hline & $\begin{array}{l}\text { Care for groups that are difficult to reach, such as ethnic minorities } \\
\text { and people with a low SEP }\end{array}$ & 28.8 \\
\hline \multirow[t]{3}{*}{ Diabetes nurses } & $\begin{array}{l}\text { Care for groups that are difficult to reach, such as ethnic minorities } \\
\text { and people with a low SEP }\end{array}$ & 59.3 \\
\hline & $\begin{array}{l}\text { Financial, legislative and regulations issues regarding care and preven- } \\
\text { tion in accordance with the Care Standard }\end{array}$ & 43.1 \\
\hline & $\begin{array}{l}\text { Lack of effective lifestyle interventions (or access to them) to provide } \\
\text { care for people with diabetes (or at increased risk for it) }\end{array}$ & 35.4 \\
\hline \multirow[t]{3}{*}{ Dieticians } & $\begin{array}{l}\text { Care for groups that are difficult to reach, such as ethnic minorities } \\
\text { and people with a low SEP }\end{array}$ & 45.7 \\
\hline & $\begin{array}{l}\text { Financial, legislative and regulation issues regarding care and preven- } \\
\text { tion in accordance with the Care Standard }\end{array}$ & 43.2 \\
\hline & $\begin{array}{l}\text { Care for specific groups, such as pregnant women and people with a } \\
\text { depression }\end{array}$ & 34.4 \\
\hline \multirow[t]{3}{*}{ Physiotherapists } & $\begin{array}{l}\text { Financial, legislative and regulations issues regarding care and preven- } \\
\text { tion in accordance with the Care Standard }\end{array}$ & 42.9 \\
\hline & $\begin{array}{l}\text { Care for specific groups, such as pregnant women and people with a } \\
\text { depression }\end{array}$ & 38.9 \\
\hline & $\begin{array}{l}\text { Care for groups that are difficult to reach, such as ethnic minorities } \\
\text { and people with a low SEP }\end{array}$ & 38.8 \\
\hline \multirow[t]{3}{*}{ Internal medicine physicians } & $\begin{array}{l}\text { Lack of effective lifestyle interventions (or access to them) to provide } \\
\text { care for people with diabetes (or at increased risk for it) }\end{array}$ & 55.0 \\
\hline & Standardized recording and exchange of information & 36.6 \\
\hline & $\begin{array}{l}\text { Care for groups that are difficult to reach, such as ethnic minorities } \\
\text { and people with a low SEP }\end{array}$ & 35.0 \\
\hline Pediatricians & Lack of refresher courses about counseling in self-management & 37.5 \\
\hline
\end{tabular}




\begin{tabular}{lll}
\hline Health care professionals & Perceived barriers & $\%$ \\
\hline & $\begin{array}{l}\text { Financial, legislative and regulations issues regarding care and preven- } \\
\text { tion in accordance with the Care Standard }\end{array}$ & 28.2 \\
& $\begin{array}{l}\text { Care for groups that are difficult to reach, such as ethnic minorities } \\
\text { and people with a low SEP }\end{array}$ & 25.6 \\
\hline
\end{tabular}

Note: multiple answers allowed

\section{Discussion}

The main aim of this study was to examine the perceptions of health care professionals regarding the Care Standard for type 2 diabetes of the Netherlands Diabetes Federation (NDF) and the barriers to its implementation. Three years after the introduction of the updated CS in 2007, only one third of the health care professionals actually possessed the CS, while $17 \%$ were totally unfamiliar with it. Previous studies found limited familiarity with clinical guidelines to be a major barrier for their implementation (Cabana et al., 1999; Francke et al., 2008). It is, however, questionable whether possession of the CS is a prerequisite for delivering high quality care. In the Netherlands, continuity of care has been introduced as part of an integrated, programmatic approach to chronic diseases, which means that professionals working in primary care are subcontracted by a larger Care Group, which is most often exclusively owned by GPs (Coordinatieplatform Zorgstandaarden, 2010; Struijs \& Baan, 2011). These Care Groups often appear to develop their own Care Standards and guidelines, but these are typically based on the CS and other evidence-based guidelines. Professionals do not always seem to be aware of this approach, which results in a low reported familiarity with the CS, whereas the care is still delivered according to the CS in routine practice (Raaijmakers et al., 2010). Involving these Care Groups in future studies assessing perceptions regarding the Care Standard, similar to studies on the Bundled Payment system in the Netherlands (Lemmens, 2012), would be very useful.

Almost two-thirds of the professionals in our survey who were to some extent familiar with the CS thought the CS contributed greatly to ensuring the quality of care, and the feasibility of working in accordance with the CS was largely endorsed. These results are in line with the findings of studies of clinicians' attitudes towards guidelines, which showed that clinicians agreed that guidelines do contribute to the quality of care (Farquhar, Kofa, et al., 2002). At present, however, Dutch health care professionals tend to perceive the guidelines issued by the own professional association as the norm for high quality diabetes care, more so than the CS. Recent research has suggested a lack of knowledge about or acceptance of more recent guidelines to be a reason for the lack of adherence to guidelines (Puder \& Keller, 2003). Only a small number of the health care professionals in our study who were to some extent familiar with the CS perceived themselves as working in complete accordance with it (15.3\%). 
The majority of our respondents perceived barriers in implementing diabetes care or in relation to working in accordance with the CS. Overall, we found a lack of effective lifestyle interventions (or access to them) to be the most important perceived barrier, although programs meeting requirements for cost-effective lifestyle interventions have been developed in the Netherlands and have been implemented in several pilot projects in primary care, examples being 'Exercise therapy' (Helmink et al., 2010) and 'Exercise on prescription' (Hosper et al., 2008). The familiarity with such programs, as well as access to them, should obviously be improved. A registration and assessment system for health education and health promotion interventions has been developed in the Netherlands in an attempt to promote quality assurance and control (Brug et al., 2010). Whether this system will help ensure that the most effective and efficient interventions are implemented and disseminated can as yet not be guaranteed (Algemene Rekenkamer, 2010), but at least a comprehensive national list is being put together. Another important perceived barrier we identified was that of financial, legislative and regulations-related issues regarding care and prevention in accordance with the CS. A recent study in the Netherlands examining the fine-tuning of care for chronic conditions also showed that facilities for prevention and self-management were not frequently reimbursed by health insurance companies because these facilities had not yet been finalized (Algemene Rekenkamer, 2010). Systematic consultations with relevant stakeholders (such as the Health Insurance Board, the Dutch Healthcare Authority and the Ministry of Health, Welfare and Sport) are needed to ensure that all elements of the CS are covered by health insurance.

We identified several differences between subgroups of our respondents in terms of working in accordance with the CS and their appreciation of the CS. The younger professionals were less likely to perceive themselves as working in complete accordance with the CS. In contrast, results from three reviews showed that young or less experienced professionals were more inclined to use guidelines than older, experienced professionals (Francke et al., 2008). The general practitioners in our study were most likely to perceive themselves as working in accordance with the CS, much more so than internal medicine physicians. Diabetes nurses were less positive about CS being the norm for high quality diabetes care than general practitioners and internal medicine physicians. As regards the feasibility of working in accordance with the CS, the general practitioners were more positive than diabetes nurses and internal medicine physicians. Internal medicine physicians were the least positive about the guidelines issued by the own professional association as the norm for high quality diabetes care and the contribution of the CS to ensuring quality of care. In contrast to these findings, a study among Norwegian professionals found general practitioners to demonstrate greater uncertainty and confusion in relation to their views on the volume, legal status, accessibility, evidence base and clarity of clinical guidelines in general, compared to other medical doctors (Carlsen \& Bringedal, 2011). An explanation for these contrasting findings may be that unlike clinical guidelines, the CS focuses on a multidisciplinary approach in which the general practitioner is 
considered to assume the role of a director of diabetes care (with the opportunity to delegate some care tasks to the practices nurses and diabetes nurses). Further external validation of our results is limited by the lack of studies comparing perceptions of guidelines between different groups of health care professionals, and by the inconsistent evidence (Carlsen \& Bringedal, 2011).

A strength of the current study is that we included a large sample of health care professionals that consisted of a broad range of professions involved in diabetes care. Since the application of the Care Standard for diabetes is unique in the Netherlands, the results of this study can inform similar future approaches in other countries.

Some limitations need to be acknowledged. Since our study sample was selfselected, it is plausible that the current sample had greater affinity and involvement in diabetes care compared to the entire population. Nevertheless, the representativeness of the sample of health care professionals is indicated by the percentage of general practitioners working in a care group (77.1\%), which is in line with the estimated national percentage (78.0\%) (Van Til, 2010). Moreover, all professions were represented in our sample in proportion to their total numbers in the Netherlands. Another limitation is that the results are based on self-reported data, which may have led to bias (e.g. through factors related to social desirability). A review comparing studies of adherence to guidelines using observational data and self-reported data showed that self-reported adherence frequently exceeded the observed adherence (Francke et al., 2008). Finally, a limited number of factors were assessed, in order to limit the burden to the respondents.

\section{Conclusions}

Familiarity with the Netherlands Diabetes Federation Care Standard (CS) for type 2 diabetes among health care professionals appears to be limited. Despite the respondents' positive judgment about the feasibility of working in accordance with the CS and about the contribution of the CS to quality assurance, they tended to perceive the guidelines issued by the own professional association as the norm for high quality diabetes care, rather than the CS. The National Diabetes Action Program would therefore be well advised to further educate health care professionals and professional organizations about the content of the CS and especially its added value relative to the guidelines for their own professional groups, in terms of the multidisciplinary approach to diabetes care. Furthermore, attention should be paid to the main perceived barriers to working in accordance with the CS. Therefore, professionals should be educated about the existence of effective lifestyle interventions and their access to these programs should be improved. In addition professionals need to be supported in providing care for groups that are difficult to reach and the financial, legislative and regulations issues relating to care and prevention in accordance with the CS. 
CHAPTER 2

\section{Abbreviations}

CCM, Chronic Care Model; NAD, National Diabetes Action Program; NDF, Netherlands Diabetes Federation; CS, Care Standard; CG, Care Group 


\section{Chapter 3}

\section{Perceived facilitators and barriers in diabetes care: a qualitative study among health care professionals in the Netherlands}

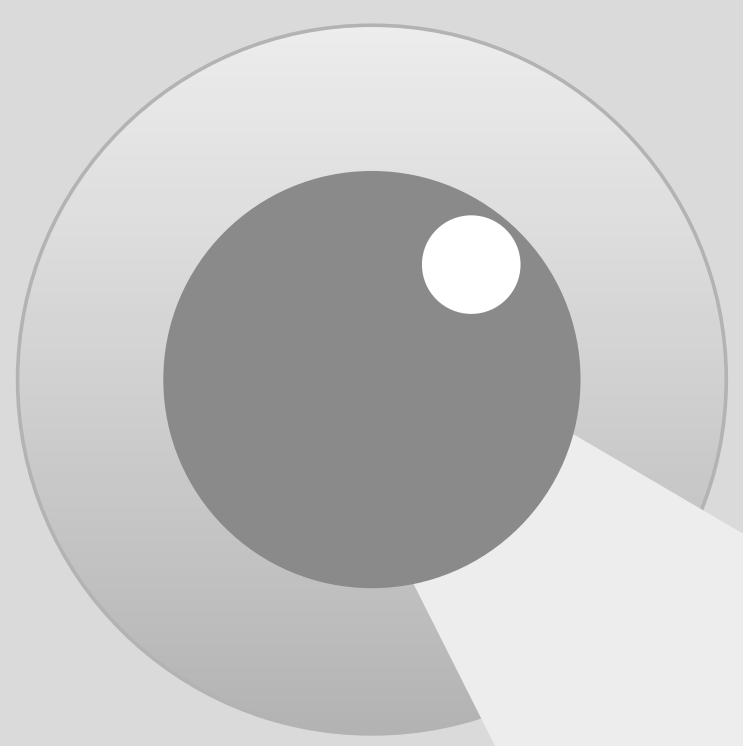

Published as: Raaijmakers, L.G.M., Hamers, F.J.M., Martens, M.K., Bagchus, C., de Vries, N.K., Kremers, S.P.J. (2013). Perceived facilitators and barriers in diabetes care: a qualitative study among health care professionals in the Netherlands. BMC Family Practice, 14(1), 114. 


\section{Abstract}

Introduction: The need to understand barriers to the implementation of health care innovations in daily practice has been widely documented, but perceived facilitators and barriers in diabetes care by Dutch health care professionals remain unknown. The aim of this study was to investigate these factors among health care professionals (HCPs) using a qualitative research design.

Methods: Data were collected from 18 semi-structured in-depth interviews with HCPs from all professions relevant to diabetes care. The interviews were recorded and transcribed verbatim and the data were analyzed using NVivo 8.0.

Results: Major facilitators were the more prominent role of the practice nurses and diabetes nurses in diabetes care, benchmarking, the Care Standard (CS) of the Netherlands Diabetes federation and multidisciplinary collaboration, although collaboration with certain professional groups (i.e. dieticians, physical therapists and pharmacists), as well as the collaboration between primary and secondary care, could still be improved. The bundled payment system for the funding of diabetes care and the role of the health insurers were perceived as major barriers within the health care system. Other important barriers were reported to be the lack of motivation among patients and the lack of awareness of lifestyle programs and prevention initiatives for diabetes patients among professionals.

Conclusions: Organizational changes in diabetes care, as a result of the increased attention given to management continuity of care, have led to an increased need for multidisciplinary collaboration within and between health care sectors (e.g. public health, primary care and secondary care). To date, daily routines for shared care are still sub-optimal and improvements in facilities, such as registration systems, should be implemented to further optimize communication and exchange of information. 


\section{Background}

Diabetes mellitus is a rapidly growing health problem, which affects approximately 366 million people worldwide (International Diabetes Federation, 2011). The prevalence of diabetes in the Netherlands in 2011 was 801.000 and this number increased by 87,000 patients each year, thereby approaching 1 million (6\% of the total population) (Baan et al., 2014). The incidence concerned 45,000 men and 42,000 women (5,5 per 1.000 men and 4,9 per 1.000 women). In addition to these diagnosed patients, registration data in general practice showed that an estimated $25 \%$ of the patients is as yet undiagnosed in the Netherlands (Rijksinstituut voor Volksgezondheid en Milieu, 2011). Reasons for this number of undiagnosed patients are amongst others the lack of attention of professionals for early symptoms of diabetes (Baan, 2009). Furthermore, data on the prevalence of diabetes in nursing homes and on the prevalence of diabetes type 2 among children and adolescents appears to be lacking (Baan, 2009).

Diabetes is a complex and systemic chronic illness, which affects various organs and systems and is often accompanied by other diseases. Hence, diabetes requires continuous medical care and ongoing patient self-management and support to prevent acute complications, like hyperglycemia or hypoglycemia and reduce the risk of complications in the long run such as hypertension, cardiovascular diseases or kidney failure (American Diabetes Association, 2011; World Health Organization, 2013). Continuity of care is concerned with the quality of health care for patients with chronic conditions like diabetes (World Health Organization, 2013). Management continuity is particularly important in chronic diseases such as diabetes, since the care for these patients requires optimal coordination and communication between the different health care professionals and organizations that contribute to the patients' care (Gulliford et al., 2007; Haggerty et al., 2003; Reid et al., 2002). Management continuity can be achieved when services are seamlessly linked and this is facilitated by shared management plans or care protocols (Haggerty et al., 2003). Many aspects of the care for diabetes patients are nowadays managed by patients themselves on a life-long basis (Barlow et al., 2002).

In recent years, multiple changes in diabetes care have been introduced in the Netherlands, with the aim of improving the continuity and quality of care. Attention to continuity of care has increased as a result of the 2008 initiative of the Dutch Ministry of Health, Welfare and Sport to start an integrated, programmatic approach to chronic diseases (Ministerie van Volksgezondheid Welzijn en Sport, 2008). The concept of continuity of care is also reflected in the Chronic Care Model (CCM) (figure 2.1), a framework that can be used to optimize the provision of care for patients with chronic conditions (Bodenheimer et al., 2002b), and that advocates integrated care and disease management and the use of evidence-based Care Standards and guidelines (Raad voor de Volksgezondheid en Zorg, 2011). The CCM focuses on improving and optimizing six key elements of the health care system: community resources and policies, organization of health care, self-management support, delivery system design, decision support and 
clinical information systems (Bodenheimer et al., 2002a). In Dutch diabetes care, the CCM is reflected in the National Diabetes Action Program (NAD), which is funded by the Ministry of Health, Welfare and Sport (Nederlandse Diabetes Federatie, 2009). The overall purpose of the NAD (2009-2013) is to create the circumstances, conditions and instruments necessary to slow down the increase in the number of people with diabetes and to reduce complications in diabetes patients (Nederlandse Diabetes Federatie, 2009).

The main objective of the action program is the systematic implementation of the Netherlands Diabetes Federation (NDF) Care Standard (CS) for the content, organization, quality and funding of diabetes prevention and care (Nederlandse Diabetes Federatie, 2009). A Care Standard is a general framework outlining the treatment of people with a specific condition, while clinical guidelines describe the content of care in more detail (Coordinatieplatform Zorgstandaarden, 2010; Seidell et al., 2012). The NDF CS for type II diabetes mellitus describes the norm for generic multidisciplinary diabetes care and focuses on the content, organization and quality of diabetes care. The CS is constantly updated and extended, and is based on evidence-based guidelines (Nederlandse Diabetes Federatie, 2013b; Struijs \& Baan, 2011). It functions as a general overarching framework for the guidelines for the individual professional groups and focuses on a multidisciplinary approach to diabetes care. In addition, the CS is used as a purchasing instrument within the Dutch bundled payment approach. In the Netherlands, insurers purchase the services and care as described in the CS from a general contractor (called the Care Group), which ends up in a so called bundled payment contract. Based on this contract, the Care Group assumes financial and clinical accountability and in turn subcontracts individual care providers (like the GP, dietician, internal medicine physician, etc.) or delivers parts of the services by itself (Struijs \& Baan, 2011). Care Standards are intended to provide health care professionals (HCPs), patients, researchers and funding bodies with a specification of the components of diabetes care, general treatment goals, and tools to evaluate the quality of care (American Diabetes Association, 2011). The Netherlands can be regarded as unique in the use of the CS for diabetes (Seidell et al., 2012). The NAD consists of five subthemes, which are in line with the concepts of the CCM and include activities to help achieve the main aim of the NAD: 'Prevention', 'Position of the patient and client', 'Quality, organization and knowledge', 'Rules and funding' and 'E-communication and ICT facilities'. For each of these themes, it formulates instrumental objectives, which are implemented in various projects (Nederlandse Diabetes Federatie, 2009).

The introduction of innovations or changes in health care is widely recognized as a complex process with several factors affecting the process positively, i.e. good communication skills of professionals, or negatively, i.e. limited time and personnel resources (Fleuren et al., 2004). Narrowing the gap between what we know and what HCPs actually do is a challenge that can help achieve effective and efficient health care (Cochrane et al., 2007). The need to understand barriers to optimal health care and the dissemination 
and implementation of health care innovations in daily practice has been widely documented (Cochrane et al., 2007; Grol \& Grimshaw, 2003).

Previous studies have identified several of these facilitators of and barriers to effective care, operating at different levels in the health care system (the patient, the individual professional, the health care team, the organization of health care or the wider environment)(Cabana et al., 1999; Grol \& Grimshaw, 2003). Patient-related barriers include patient characteristics, such as lack of knowledge about diabetes (Goderis et al., 2009; Wens, 2005), lack of motivation to change (Brown et al., 2002; Goderis et al., 2009), low adherence (Cochrane et al., 2007; Goderis et al., 2009), and a need for education (Brown et al., 2002). Patient-related facilitators that have been found are early educational interventions at the start of the illness and patients' ability to be responsible for and have control over their diabetes (Wens, 2005). Examples of professional-related barriers are lack of motivation(Van Bruggen et al., 2008), lack of appropriate peer influence (Cochrane et al., 2007), lack of knowledge (Cochrane et al., 2007; Goderis et al., 2009; Van Bruggen et al., 2008), not reading guidelines (Dijkstra et al., 2000), lack of confidence in clinical skills (Brown et al., 2002; Nam et al., 2010), lack of effective communication tools, and lack of counseling and shared decision-making skills (Nam et al., 2010). Professional-related facilitators include good communication skills that help to check patients' needs and not overload them with information (Wens, 2005) and continuing medical education of physicians (Brown et al., 2002). Barriers related to the health care team include suboptimal communication between HCPs (Kripalani et al., 2007; Pantilat et al., 2002), the lack of clear descriptions of professionals' responsibilities within the team (Goderis et al., 2009), and ignoring responsibilities of fellow professionals (Wens, 2005). Additional barriers are the need for identical messages to the patients from all HCPs and competition between specialists and family physicians (Goderis et al., 2009) Working in a multidisciplinary team has been reported to be a facilitating factor associated with the health care team (Wens, 2005) as well as the beneficial effects of electronic data interchange on the frequency of communication between general practice and hospitals (Branger et al., 1998). Examples of barriers in the organizational context are financial disincentives such as the lack of reimbursement (Dijkstra et al., 2000; Goderis et al., 2009; Grol \& Grimshaw, 2003; Van Bruggen et al., 2008); organizational constraints such as the absence of organizational systems to support diabetes management (i.e. registries, automatic recall systems and reminder systems) (Goderis et al., 2009); and the lack of an individualized plan of care (Nagelkerk et al., 2006). Organization-related facilitators are the importance of adhering to clinical practice guidelines and allocating time for patient education (Brown et al., 2002). Examples of wider environmental characteristics that negatively influence guideline implementation in health care include limited time and personnel resources or services available for special populations such as the elderly and ethnic minorities (Brown et al., 2002; Dijkstra et al., 2000) as well as work pressure (Cabana et al., 1999; Chesover et al., 1991; Dijkstra et al., 2000; Francke et al., 2008). Support from managers, including financial support to create opportunities 
to participate in educational meetings or to arrange the necessary materials or aids, and active involvement of superiors in the implementation process, have been reported as facilitators (Sachs, 2006).

However, little is as yet known about the facilitators and barriers perceived by Dutch HCPs in diabetes care. This is of special interest since the Netherlands can be regarded as one of the frontrunners in implementing continuity of care and the CS. Therefore, the aim of this study was to investigate these facilitating and impeding factors among HCPS using a qualitative research design.

\section{Methods}

\section{Participants}

Data were collected from 18 semi-structured interviews with health care professionals held between November 2010 and January 2011. Participating professionals were selected based on their primary role in diabetes care, as described in the Care Standard (Nederlandse Diabetes Federatie, 2013b). Participants were randomly selected from a database of a previous quantitative study we conducted among health care professionals in the Netherlands (Raaijmakers et al., 2010). In the questionnaire of that particular study participants were asked whether they were willing to participate in future research on the topic. The random selection of participants for our study resulted in a sample that was geographically dispersed in the Netherlands and professionals were not collaborating with each other. Consequently, selected participants were contacted by telephone and invited for participation and an appointment for an interview at their professional office was made. Participants included family physicians (FNs; $n=3$ ), practice nurses (PNs; $n=3$ ), diabetes nurses (DNs; $n=2$ ) (one in primary care, one in secondary care), dieticians $(D I ; n=3)$ (two in primary care, one in secondary care), physical therapists (PTs; $n=2$ ) (primary care), internal medicine physicians (IPs; $n=3$ ) and pharmacists (PAs; $n=2$ ). The mean age of the participants was 43.9 years (range 31-59), 39\% were male and 67\% were working in primary care. Ethical approval for this study was not needed under Dutch law.

\section{Interview procedure}

The interviews were semi-structured, with open-ended questions, and followed an interview guide based on the main theme of 'Care Standard' and the subthemes of the NAD. Each interview took place at the professional's office and lasted approximately one hour. All interviews were audiotaped and transcribed by the interviewer (LR). The interview guide included the following themes: 'background and function in diabetes care', 'general appreciation of diabetes care', 'organization of diabetes care', 'continuity of 
care', 'funding of diabetes care and the role of the health insurer', 'the CS', 'working in accordance with the CS', 'individual care plan', 'multidisciplinary collaboration in diabetes care', 'standardized registration and exchange of information', 'prevention and lifestyle interventions', 'benchmarking and health care quality', 'barriers to diabetes care and to working in accordance with the CS' and 'facilitators of diabetes care and of working in accordance with the CS'. Some examples of questions were: 'What do you think of the organization of diabetes care in the Netherlands?', 'What is the role of the health insurers in diabetes care?' and 'What do you think of the exchange of information and communication about patient care between health care professionals in your care team?'

\section{Research model}

We used the Chronic Care Model (CCM) (Bodenheimer et al., 2002b) (figure 3.1) to classify the facilitators and barriers into the following key elements of the health care system: community resources and policies, the way health care is organized, selfmanagement support, delivery system design, decision support and clinical information systems (Bodenheimer et al., 2002a). We added the category of HCP-related factors to the model. Community resources refer to the need among provider organizations for linkages with community-based resources, e.g. exercise programs and senior centers, in order to improve chronic care. The element of organization of health care is concerned with the structure, goals and values of a provider organization and its relationship with purchasers, insurers and other providers. Self-management support involves collaboratively helping patients and their family to manage their chronic condition by acquiring the right skills, providing self-management tools and routinely assessing problems and accomplishments. The delivery system design refers to the creation of practice teams with a clear vision on the planned management of chronic conditions within the structure of the medical practice. Decision support is concerned with the integration of evidence-based standards and guidelines in daily practice. Clinical information systems involve computerized information which helps care teams comply with practice guidelines and standards, provide feedback to physicians and serve as records to assist planning individual patient care and conducting population-based care (Bodenheimer et al., 2002a). Finally, HCP-related factors refer to factors concerned with the individual health care professional, such as their motivation, knowledge of and affinity with diabetes care. 


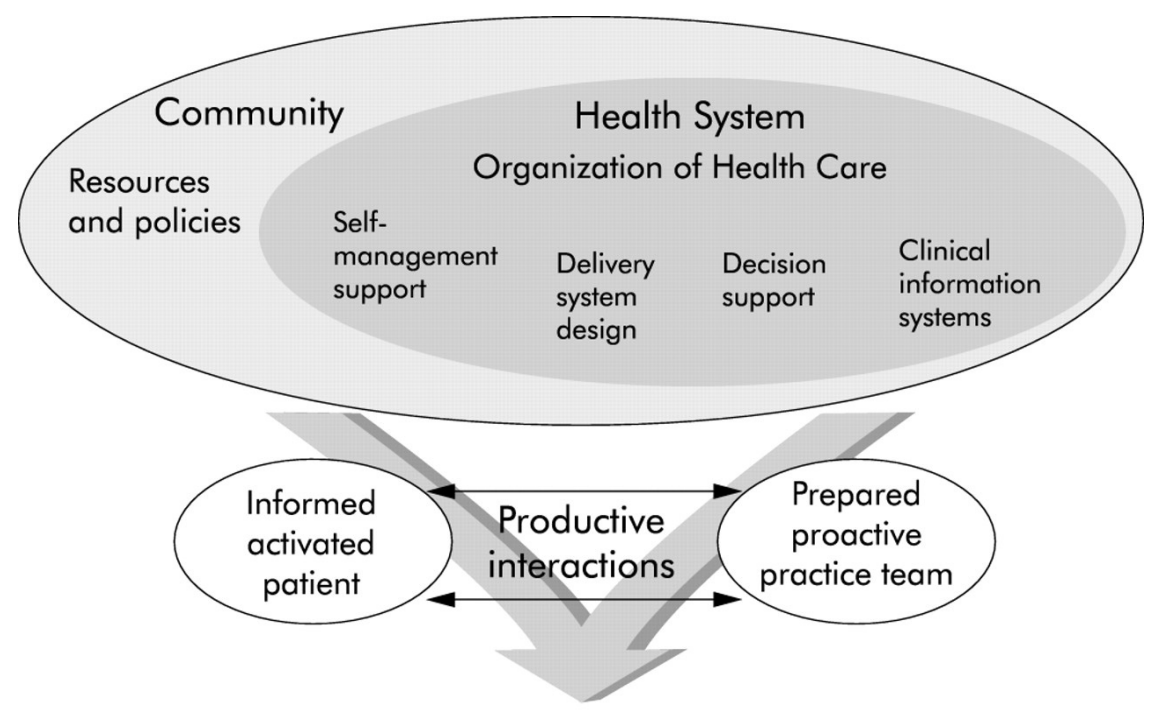

Functional and clinical outcomes

Figure 3.1: The Chronic Care Model

\section{Data analysis}

Data were analyzed using the NVivo qualitative research software package, version 8.0. Meaning units (words or sentences) were labeled with codes and the first researcher (LR) grouped these codes into categories and subcategories. The coding scheme was derived from the CCM.). The codes were checked by and discussed with an independent co-researcher (FH) and disagreements were solved in a consensus meeting. In case of unsolved disagreements between LR and FH, a third researcher (SK) was consulted for a final decision. In case data did not fit with the CCM, they were grouped in additional categories where necessary.

\section{Results}

Box 3.1: List of abbreviations for HCPs
FP: Family physician
PN: Practice nurse
DN: Diabetes nurse
DI: Dietician
PT: Physical therapist
IP: Internal medicine physician
PA: Pharmacist 


\section{Community resources and policies}

One of the facilitating factors perceived by several HCPS (PN1, DN1, PT1, IP1) in the community was the increased attention to diabetes in health care and the media. In addition, one respondent (DI1) stated that diabetes is a popular topic in scientific research as well. One respondent (DI1) thought it was an impediment that diabetes is often seen as a disease that a lot of people suffer from and that it is therefore not taken seriously enough (see table 3.1 in additional file for quotes).

The majority of the respondents reported not to be aware of lifestyle programs and prevention initiatives that they could refer their diabetes patients to. One respondent (PN2) reported not to have a list of local exercise facilities and another respondent (PT2) agreed that they have to look for opportunities in the neighborhood themselves using their personal network. However, two respondents (FP1, PN2) reported that many lifestyle programs were available, but the problem was that patients often relapse soon when they have to maintain their new lifestyle after the lifestyle program has ended. Another respondent (FP2) reported that their care group had drawn up a list of local initiatives.

\section{Organization of health care}

In relation to continuity of care, the majority of the respondents mentioned the role of the practice nurses and diabetes nurses as a very important improvement in diabetes care since they are up to date on the most recent developments, have more time and are trained to organize their care efficiently. However, two HCPS (PN2, DN2) reported the possible decreasing expertise of FPs as a negative side effect of this substitution of care. Two respondents (PN1, PN3) mentioned that many changes have occurred in diabetes care and that it takes time to get used to these changes.

The introduction of the first CS in 2003 coincided with the development of the 'bundled payment' approach for integrated chronic care (Struijs \& Baan, 2011). The majority of the interviewees perceived the bundled payment system as a barrier to diabetes care because it is not suitable for chronic conditions; it leads to egoism and higher costs and makes care less transparent. One respondent (PN2) perceived the bundled payment system as beneficial since the funding system used to be very fragmented, while the overall picture is very clear nowadays.

The majority of the respondents reported the funding of diabetes care and more specifically the role of the health insurers as an important barrier. Four respondents (PN2, DI1, IP1, PA1) mentioned that the health insurers have a lot of influence and are dominant, while three other respondents (FP1, FP2, IP2) stated that the collaboration with health insurers is very inflexible. One respondent (FP2) perceived the role of the health insurers also as a facilitating factor, since they have influenced the development of multidisciplinary care groups in a very positive way. 


\section{Self-management support}

Overall, the professionals perceived the lack of motivation among patients to be hampering the delivery of their care. Additionally, one respondent (PN1) reported that patients are unaware of the importance of self-management in diabetes, and another respondent (IP3) thought that patients' own sense of responsibility is disappointing. However, one respondent (PN3) perceived a tendency towards self-management among patients and another respondent (PN2) mentioned education as an important tool to increase compliance by patients. Two respondents (FP2, PN1) also reported to have experienced specific problems with hard-to-reach groups such as low SES patients or patients from ethnic minorities.

Several respondents (FPs, DI, PT2) mentioned the added value of the individual care plan to motivate patients and its contribution to self-management by patients. However, some respondents (FP3, PN3, IP1, DI2) doubted whether such a plan is suitable for the average diabetes patient, who is not as motivated, independent and able to manage their diabetes as is assumed in the care plan.

\section{Delivery system design}

The majority of the HCPs perceived multidisciplinary collaboration to be effectively organized both within primary care and between primary and secondary care, and one respondent (DN2) also perceived good collaboration within secondary care. Several respondents (FP1, PN, DI3, PT1, PA2) perceived the direct communication lines and short (physical) distances to other professionals as facilitating the achievement of multidisciplinary collaboration in diabetes care. Furthermore, one respondent (PA) mentioned systematic consultations with other professionals as beneficial to collaboration.

The respondents also perceived barriers in relation to multidisciplinary collaboration. Some respondents would like to collaborate more with specific professional groups such as dieticians, physical therapists and pharmacists. One respondent (DN1) reported that professionals in secondary care assume that they are collaborating better than professionals in primary care, and that health care needs to get rid of the island culture in order to improve multidisciplinary collaboration. Two respondents $(1$, IP3) thought that you need to have one or two leaders who maintain the collaboration and that systematic regional consultation is desirable to improve the collaboration.

\section{Decision support}

Overall, the interviewees perceived the CS to involve all facets of diabetes care and to contribute to the quality of diabetes care. The CS is a solid agreement we made as professionals (DN1); it offers a clear framework to check whether you have everything (IP3) and provides unequivocal clarity (DI3). However, one respondent (IP1) perceived the lack of practice-based working as a barrier and two respondents (IP1, IP2) reported that a risk 
of tunnel vision as a result of the CS and its sanctioning character were barriers. One of the dieticians (DI2) mentioned that the CS describes everything comprehensively, except the part in which the dietician is involved. One of the internal medicine physicians (IP1) reported that the use of protocols in care has led to the provision of care in accordance with agreements, but policy makers think that this automatically implies high quality care, which is a mistake.

With regard to the implementation of the CS, one respondent (IP1) reported that the CS is either insufficiently promoted or is known as a forcing model. Another respondent (FP2) reported the need for time to adapt to working in accordance with the current version of the CS, before a new update is introduced.

\section{Clinical information systems}

Overall, HCPs perceived the availability and use of a large number of different registration systems as a major barrier in terms of registration and exchange of information in diabetes care. These systems are used by the so called Care Groups, practices and hospitals in the Netherlands to register patient information and health care quality indicators. HCPs perceived these systems often as incompatible, which impedes communication. One respondent (PN3) indicated the use of the same registration system by all professionals involved, as well as the use of digital patient records as facilitating factors in improving the quality of care.

The majority of the respondents perceived the use of the principles of benchmarking as a positive development in diabetes care. Benchmarking was reported as a positive feedback mechanism and stimulating factor (FP3), it makes professionals more aware (DI1) and it is helpful in the communication with health care insurers (IP2). On the other hand, three respondents (FPs, PT2, PA2) perceived disadvantages of external benchmarking (by health insurers). In addition, the majority of the respondents reported that the quality of the indicators used for benchmarking acted as a barrier. According to the respondents, several indicators are nonsensical or manipulated, and certain aspects of their care are not covered by the indicators. Moreover, two respondents (IP2, PN3) mentioned that health insurers are interested in other indicators than those that are regarded as most important by the professionals. One respondent (IP1) argued that the use of the current indicators and benchmarking principles leads to manipulation of information.

\section{HCP-related factors}

Barriers and facilitating factors with regard to HCPs were related to their training, professional vision, image and affinity with diabetes care. Two respondents (PN3, DN1) reported the high educational and knowledge levels of Dutch health care professionals as a facilitating factor. However, two participants (PN2, IP3) perceived the lack of expertise 
among FPs as an impediment, and another HCP (IP1) reported that colleagues still use unqualified staff for the tasks of practice nurses and diabetes nurses.

The respondents perceived barriers in relation to the role and image of dieticians and physical therapists. Both of the dieticians working in primary care whom we interviewed felt that they had a negative reputation among patients. Dieticians used to be seen as people who provided rules about what people were allowed and especially not allowed to eat, and this image still prevails among patients, making them unwilling to consult dieticians. One of the participating FPs (FP2) confirmed that this reputation also existed among professionals. Several respondents took the view that physical therapists need to be aware of their role in diabetes care. One of the participating physical therapists (PT1) held the opinion that the role of their profession in diabetes care is too limited, and emphasized that their role is mainly concerned with eliciting behavioral change in patients.

Two respondents (PN1, DN2) reported that the lack of affinity with diabetes in HCPs was a barrier. In Dutch health care, family physicians are automatically involved in the care for patients with type 2 diabetes and a high risk of diabetes. These participants argued that FPs do not always have affinity with diabetes care because of their more general function and therefore diabetes care should preferably be provided by FPs with an additional education in diabetes, which is available to in the Netherlands.

\section{Discussion}

This study has contributed to our understanding of facilitators and barriers perceived by Dutch health care professionals in diabetes care. Using the CCM to identify such barriers and facilitators from the perspective of health care professionals resulted in a structured overview of these factors. One major facilitator we found was the more prominent role that practice nurses and diabetes nurses in diabetes care have been given since the introduction of continuity of care and more specifically management continuity in the Netherlands, and which is greater than in other health care domains. These nurses can play an important role in educating patients and encouraging adherence; and in certain situations they can even replace physicians in delivering many components of diabetes care (Ubink-Veltmaat et al., 2005). Moreover, a recent study of Dutch diabetes care found that standardized diabetes care, delivered by a nurse specialized in diabetes, is a good alternative to standard care by an internal medicine physician, with comparable results after one year in terms of treatment goals, and even better results in terms of patient goals and cost-effectiveness (Houweling et al., 2009). In contrast, the role and image of dieticians and physical therapists were reported as barriers. Dieticians have (or perceive themselves as having) a negative reputation among patients and fellow professionals. A previous Dutch study also reported the image of the dietician to be a barrier to the collaboration with family physicians (Mathus-Vliegen, 2006). The role of physical 
therapists in diabetes care seems somewhat unclear and too limited in the opinion of the physical therapists themselves.

Another major facilitator is multidisciplinary collaboration, although the collaboration with certain professional groups (i.e. dieticians, physical therapists and pharmacists) could be further improved, as could the collaboration between primary and secondary care. In line with our results, a previous study among Belgian family physicians reported the competition between specialists and family physicians to be a barrier to evidencebased diabetes care (Goderis et al., 2009). Our respondents also perceived the large number of different registration systems to record and exchange information in diabetes care as a major barrier to collaboration and communication. Previous studies reported suboptimal communication between HCPs to be a major problem in relation to shared care (Kripalani et al., 2007; Pantilat et al., 2002) and the beneficial effects of electronic data interchange on the frequency of communication between general practice and hospitals (Branger et al., 1998).

Our respondents perceived the CS as a facilitator, since they perceived it to be a clear framework that contributes to quality of care. This is in agreement with the findings of studies on clinicians' attitudes towards guidelines, which showed that clinicians agree that guidelines contribute to the quality of care (Farquhar, Kofa, et al., 2002).

Benchmarking was also perceived as a major facilitating factor. Previous studies have concluded that benchmarking is a promising tool for quality improvement in chronic care in general, and in diabetes care specifically, but until now there has been a striking lack of clinical evidence from controlled trials (Nobels et al., 2011). However, the majority of our interviewees reported the quality of the indicators for benchmarking to be a barrier. A recent report on the development of European quality indicators for primary diabetes prevention programs stated that even though it is not possible to develop an error-free measure of quality, an indicator should always be tested for feasibility, reliability and validity during its development phase. Furthermore, professionals and health care insurers should always keep in mind that indicators just indicate and that they probably never completely capture the quality of the health care system. Even the best indicators have limitations, and these limitations should be taken into consideration when drawing conclusions based on the indicators (Pajunen et al., 2010).

The bundled payment system for the funding of diabetes care and the role of the health insurers were perceived as major barriers within the health care system. Our respondents thought that the bundled payment system is unsuitable for chronic conditions and is counterproductive. However, early results from the adoption of bundled payment for diabetes care in the Netherlands show that it has improved the organization and coordination of care and has led to better collaboration between health care professionals and better adherence to care protocols (De Bakker et al., 2012). Moreover, many services and health care providers seem positive about the organizational improvements in care resulting from the introduction of the bundled payment approach (Struijs et al., 2010). 
Another important barrier perceived by our respondents was the lack of motivation on the part of patients, which is consistent with the findings of previous studies reporting a lack of motivation to change among patients (Brown et al., 2002; Goderis et al., 2009). By contrast, our respondents perceived the use of individual care plans to be a facilitator contributing to self-management by patients. A previous study among Canadian physicians identified the individual care plan as a tool to achieve improved communication in the transition from specialist to primary diabetes care (Brez et al., 2009). Despite the reported benefits of individual care plans, our respondents doubted whether such plans are suitable for the average diabetes patient, who is not as motivated, independent and able to manage their diabetes as assumed in the care plan. This finding is similar to the results of a study among Belgian family physicians, who questioned the feasibility and desirability of implementing clinical guidelines in an older diabetic population (Goderis et al., 2009).

The main barrier in relation to community resources was reported to be the lack of awareness of lifestyle programs and prevention initiatives for diabetes patients among professionals. Programs meeting requirements for cost-effective lifestyle interventions have been developed in the Netherlands and have been implemented in several pilot projects in primary care, examples being 'Beweegkuur' (Exercise therapy) (Helmink et al., 2010) and 'Exercise on prescription'(Hosper et al., 2008). The familiarity with such programs, as well as access to them, should obviously be improved. In an attempt to promote quality assurance and control, a registration and assessment system for health education and health promotion interventions has been developed in the Netherlands (Brug et al., 2010). Whether this system will help ensure that the most effective and efficient interventions are implemented and disseminated can as yet not be guaranteed (Brug et al., 2010), but at least a comprehensive national list is being put together.

Some strengths and limitations of the current study remain to be addressed. A strength of this study is the use of semi-structured interviews which provide the opportunity to collect in-depth information and understand perspectives and experiences of participants. Moreover, by using the CCM to categorize the identified facilitators and barriers, we increased the standardization of reporting facilitators and barriers in relation to diabetes care. Our results show that expansion toward better integration of prevention and health promotion in the CCM would be useful (Barr et al., 2003). Furthermore, all interviews were conducted by the same researcher, in order to increase consistency in the data collection process, and all codes were checked independently by two researchers to increase conformability (objectivity and neutrality).

A limitation of the current study is the selectivity of the sample; since participation was voluntary and recruitment was conducted through a random selection of professionals from a database of a previous study on the same topic, it is plausible that the current sample had greater affinity with and involvement in diabetes care than the population of all Dutch care providers, which may limit the generalizability of our findings. Furthermore, we selected a heterogeneous sample of health care professionals 
because of their primary role in diabetes care and in relation to the multidisciplinary approach in Dutch diabetes care. However, the consequence of including this heterogeneous group was that we were only able to include 2 or 3 professionals per group and cannot guarantee that saturation has been achieved. Furthermore, we retrieved divergent views on some aspects of care and due to the relatively small sample size per professional group, we were not able to obtain consensus on all issues. Additionally, the current study is primarily focused on the organization of diabetes care and the use of the Care Standard in the Netherlands. Our results can however inform and support similar future approaches to organize diabetes care in other countries as well.

\section{Conclusion}

The substitution of care originally provided by family physicians and specialists to practice nurses and diabetes nurses seems to work out well. These organizational changes in diabetes care, as a result of increased attention given to continuity of care, have led to an increased need for multidisciplinary collaboration within and between health care sectors (including public health, primary care and secondary care). A new CS could be helpful in this respect. To date, daily routines for shared care are still sub-optimal and facilities such as registration systems should be improved to further optimize communication and exchange of information. 


\section{Additional file}

Table 3.1: Perceived facilitators and barriers in diabetes care

\begin{tabular}{l}
\hline Category of factors Quotations to illustrate the factors identified \\
\hline Community resources and policies \\
\hline $\begin{array}{l}(+) \text { 'Diabetes is receiving a lot of attention in primary and secondary care and also from } \\
\text { politics, which has led to an improvement of the care' } \\
(+) \text { 'There's a lot of attention for diabetes in the media, which makes professionals and } \\
\text { patients aware of the seriousness and that's a good thing' } \\
(+) \text { 'Diabetes is a popular topic in scientific research' } \\
(-) \text { 'Diabetes is often seen as a common disease that a lot of people have and is therefore } \\
\text { not taken seriously enough' }\end{array}$
\end{tabular}

Prevention and lifestyle interventions
(+) 'We've made a list of local initiatives with our care group, so I know where to find them'

$(+)$ 'I received an overview from the municipal Sports Service listing facilities in the neighborhood, which is very convenient'

(-) 'We don't have a local list of exercise facilities'

(-) 'There are so many programs for diabetes patients, but when the programs stop, the patients have to continue on their own and often they relapse soon'

(-) 'It's often the case that family physicians don't know where to refer people for lifestyle changes'

(-) 'You have to look for programs and possibilities in the neighborhood yourself, using your personal network' 
Role of health insurers
(+) 'The health insurers do what they have to do and the prices are established by the Dutch Healthcare Authority (government institution)'

$(+)$ 'Health insurers have influenced the development of multidisciplinary care groups in a very positive way'

$(-)$ 'The health insurers are very restrictive in the services and medication they reimburse, which leads to a decrease in the quality of care delivered'

(-) 'In our current health care system, the health insurer is dominant'

$(-)$ 'The collaboration with the health insurers is very inflexible'

$(-)$ 'The conversations with the health insurer take a lot of time and effort because of the competing interests'

(-) The health insurers have great power and that's a problem because they think about money first and quality second'

\section{Self-management support}

Motivation

$(+)$ 'If you provide patients with enough education and you get to know the individual patient, their compliance greatly improves'

$(+)$ 'There is a tendency toward more self-management by patients and that's very good'

(-) 'The problem in health care is to motivate patients'

(-) 'Patients' own sense of responsibility is disappointing'

(-) 'A lot of patients are fairly passive and dependent on medication'

(-) 'The majority of the patients practice self-management, but some are still unaware of its importance in diabetes'

Individual care plan $(+)$ 'I think it's a positive development that patients are guided towards self-management' (+) 'I absolutely see the added value of individual care plans to motivate patients'

$(+)$ 'If it works out I completely support the use of individual care plans since your patients become more motivated and take responsibility for their own health'

(-) 'It's good to make patients the masters of their own disease, but the problem is that the average type 2 diabetes patient is not the patient described in the care plan' (-) 'The problem is that we have a lot of different patient groups and it's impossible to make different individual care plans for each group'

(-) 'I think a lot of patients still adopt a dependent attitude toward their doctor and don't want an individual care plan'

Difficult to reach (-) 'We encounter specific problems because our practice is located in a low SES neighgroups borhood'

$(-)$ 'Consultations that involve an interpreter take at least a third of the usual time extra'

$(-)^{\prime}$ The problem is to motivate female migrants to become physically active'

Delivery system design

Multidisciplinary $\quad(+)$ 'In this region the collaboration between primary and secondary care is pretty well collaboration organized'

$(+)$ 'We work in a multidisciplinary team on the same floor, so we can easily ask each other things'

$(+)$ 'The short physical distances and direct communication lines to other professionals make it easy to have frequent contacts'

$(+)$ 'Systematic consultations with other professionals are beneficial for the collaboration'

(-) 'There's some arrogance among secondary care professionals because they assume they are collaborating better inside the hospital than we can in primary care' 
(-) 'We've been trying to get rid of the island culture for 10-15 years now'

(-) 'The role of the dieticians should be bigger; they're actually a very important factor'

(-) 'There should be a whole team surrounding the patient, but that's what I still feel is missing at the moment'

(-) 'I think the collaboration between the family physicians and other primary care partners such as dieticians, physical therapists and pharmacists could be greatly improved'

(-) 'You need one or two leaders who bring everyone together; when they withdraw, the continuity of care falls apart'

\begin{tabular}{ll}
\hline Decision support & \\
\hline Care Standard & $(+)$ 'I think the CS covers all facets of diabetes care' \\
$(+)$ 'The CS is a solid agreement we make as professionals' \\
$(+)$ 'It contributes to high quality care and provides a good basis for all disciplines involved' \\
$(+)$ 'It offers a clear framework and you can check whether you have everything' \\
$(+)$ 'The CS provides unequivocal clarity, so you know your colleague is doing the same' \\
\\
$(-)$ 'In essence, the CS is fine, but I would suggest to work to some extent practice-based \\
as well as evidence-based' \\
$(-)$ 'My professional group is afraid that the CS is going to dominate the care process and \\
lead to tunnel vision and barriers' \\
$(-)$ 'The problem with the CS is that you're not going to read it until you have patients to \\
whom it applies' \\
(-) 'A barrier could be that the CS is seen as sanctioning' \\
$(-)$ 'Everything is described in detail, except the part for the dietician'
\end{tabular}

Implementation of (-) 'I think it's important to promote the CS as a framework to check whether your care is CS within the right parameters, but it shouldn't be used as a forcing model'

(-) 'Give professionals some time to adapt to working with the CS; the previous version was only 3 years old'

(-) 'More attention for the CS from professional organizations would be useful'

Clinical information systems

Bench-marking $\quad(+)$ 'Transparency is very important and if you show this, health insurers notice it and act upon it'

$(+)$ 'I think the benchmark as a feedback mechanism and stimulating factor is good'

$(+)$ 'I think it can improve the care for patients since it makes you aware'

$(+)$ 'The principles of benchmarking are very good, but you need to make sure that you can use the information that professionals need to record anyway'

$(+)$ 'I think your professional group should take care of good quality, so the internal benchmark is a good thing. External could be good, but you need to be careful since the information is easily misinterpreted'

(-) 'Certain indicators, such as blood pressure, would always be good, but for other indicators you should be careful in interpreting them and they should ideally involve information like the neighborhood in which patients are living'

(-) 'A lot of basic care is not covered by indicators'

(-) 'The most important disadvantage is that they may be misused for financial settlements. That's a bad development as regards transparency'

(-) 'The information is used to manipulate in ways that are very counterproductive'

(-) 'At the moment we use indicators that are nonsensical, and other indicators are manipulated' 


\begin{abstract}
Standardized regis- $(+)$ 'We have a system in which you can record everything regarding the patient, and tration and ex- other professionals can see the information that's relevant to them'

change of infor- $\quad(+)$ 'Working with the electronic patient record system has increased the quality of our mation care'
\end{abstract}

(-) 'The systems used by different professionals are not compatible, so you can't communicate through the systems'

(-) 'After referral to secondary care there is stagnation of information provision, because of different registration systems'

(-) 'At the moment there are 10-15 different systems in use'

(-) 'Each hospital has its own system and the systems don't correspond'

(-) 'One uniform system for al family physicians would be perfect, but that's an illusion'

(-) 'The exchange of information between pharmacists and hospitals is fraught by lots of mistakes'

\title{
HCP-related factors
}

\begin{tabular}{l}
\hline Education \\
$(+)$ 'I think all Dutch health care professionals are well-trained' \\
$(+)$ 'We should grade the knowledge level of professionals in diabetes care as 8 out of 10; \\
it's one of their strong points' \\
(-) 'I see that family physicians make decisions based on insufficient knowledge' \\
(-) 'Unfortunately the entire family physician training program includes only two days \\
devoted to diabetes'
\end{tabular}

Image

$(+)$ 'The dietician's contribution is well acknowledged'

(+) 'Dieticians have a very important role to play in diabetes care'

(-) 'We family physicians agree that the reputation of dieticians needs to be improved, as the general view is that they only say what you're not allowed to do'

(-) 'Dieticians used to have a negative reputation, so patients still don't want to be referred to them, while our daily practice has changed a lot'

(-) 'Physical therapists need to be aware that they have a limited role in diabetes care because consulting a physical therapist is not necessary for all patients'

(-) 'Physical therapists need to be aware that their role not only concerns getting patients to become physically active, but also eliciting behavioral change in patients'

(-) 'The role we physical therapists are allocated in diabetes care is too limited'

Affinity (+) 'At a health center you can distribute the fields of interest, and those professionals who have an affinity with diabetes care keep up to date in that field'

(-) 'The lack of affinity with diabetes among professionals working in one-doctor practices has a negative influence on the quality of their care'

(-) 'Family physicians have less affinity with patient self-management and education than the practice nurses'

(+) facilitators, (-) barriers 



\section{Chapter 4}

\section{Correlates of perceived self-care activities and diabetes control among Dutch type 1 and type 2 diabetics}

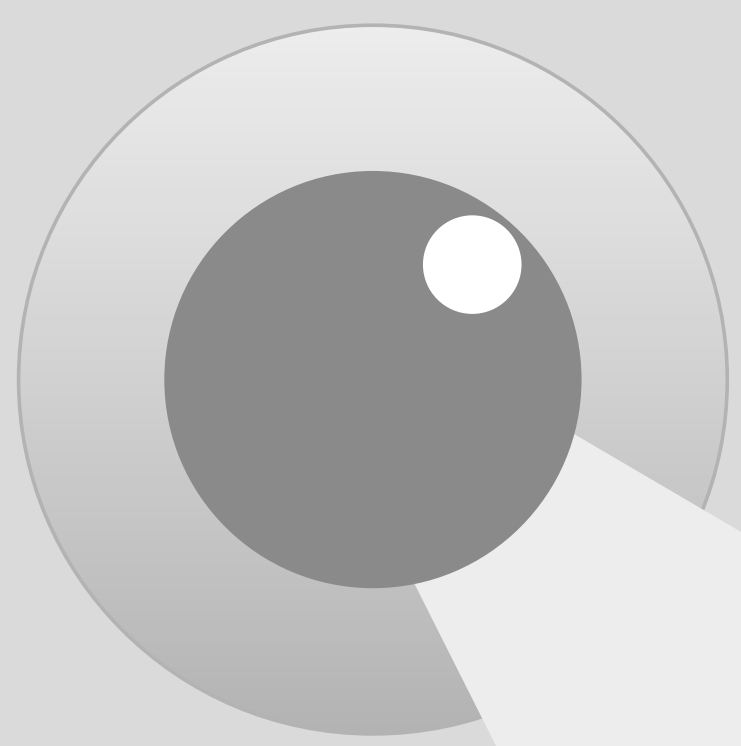

Resubmitted for publication as: Raaijmakers, L.G.M., Martens, M.K., Bagchus, C., de Weerdt, I., de Vries, N.K., Kremers, S.P.J. Correlates of perceived self-care activities and diabetes control among Dutch type 1 and type 2 diabetics. 


\section{Abstract}

This study examined how Dutch type 1 and type 2 diabetes patients' perceived autonomy support, as well as their perceived competence and treatment self-regulation, are associated with their diabetes self-care activities (healthy diet, physical activity, monitoring blood glucose, medication use) and general diabetes control. A cross-sectional questionnaire study was conducted among 143 type 1 diabetics and 384 type 2 diabetics. Overall, participants felt competent, supported in their autonomy, and perceived to autonomously self-regulate their diabetes. Our results underline the importance of perceived competence in type 1 and 2 diabetics, as this was strongly associated with adhering to a healthy diet and general diabetes control. Our findings also emphasize the need for autonomy supportive health care professionals in diabetes care. However, perceived competence appears to mediate the influence of autonomy support, especially in type 1 diabetics, while in type 2 diabetics' autonomy support remains to have a statistically significant explanatory contribution. 


\section{Introduction}

Diabetes mellitus is a rapidly growing health problem, which affects approximately 371 million people worldwide (International Diabetes Federation, 2012). The prevalence of diabetes in the Netherlands in 2011 was 801.000 and this number increased by 87,000 patients each year, thereby approaching 1 million (6\% of the total population) (Baan et al., 2014). In addition to these diagnosed patients, registration data in general practice showed that an estimated $25 \%$ of the patients is as yet undiagnosed in the Netherlands (Rijksinstituut voor Volksgezondheid en Milieu, 2011). Of the patients, 90\% is diagnosed with type 2 diabetes and the remaining percentage suffers from type 1 diabetes (Baan et al., 2014). Diabetes is a complex chronic illness, since it affects various organs and systems and is often accompanied by other diseases. Diabetes requires continuous medical care, ongoing self-management by the patient and support to prevent acute complications, like hyperglycemia or hypoglycemia and reduce the risk of complications in the long run, such as hypertension, cardiovascular diseases or kidney failure (American Diabetes Association, 2013; World Health Organization, 2013).

Many aspects of the care for diabetes patients are nowadays managed by patients themselves on a life-long basis (Barlow et al., 2002; Reed et al., 2003). As a result, the responsibility for day-to-day disease management is shifting from health care professionals to the individuals, which places a considerable burden on these patients (Barlow et al., 2002; Reed et al., 2003). Diabetes self-care involves a range of activities such as self-monitoring of blood glucose, eating a healthy diet, being physically active, taking the recommended medication and consulting health care professionals (Toobert, 2000). Dutch (EADV \& NAD, 2012) and international recommendations (American Diabetes Association, 2013) advise daily self-monitoring of blood glucose for patients with type 1 diabetes and for patients with type 2 diabetes who use insulin therapy. Since there is no evidence that self-control in type 2 patient without insulin treatment is beneficial for clinical outcomes, daily self-monitoring in these patients can be useful when patients are pregnant or have disordered blood glucose levels (EADV \& NAD, 2012). Selfmanagement skills have an important role in optimal diabetes control (Malanda et al., 2012). Positive effects of self-management training have been shown on self-monitoring of blood glucose, dietary habits, HbA1c levels and glycemic control in type 2 diabetics (Malanda et al., 2012; Norris et al., 2001). Next to their responsibilities concerning periodical physical examination and medication adjustments, health care professionals are advised to promote optimal self-management among their diabetes patients (Nederlandse Diabetes Federatie, 2013b). In order to inform counseling and treatment, there is a need to identify primary determinants of self-management among diabetes patients. Patients with type 1 and type 2 diabetes may differ in their needs regarding selfmanagement and treatment since these illnesses are different in etiology, management and prognosis. To our knowledge, however, no previous studies assessed differences between both patient groups in this respect. 
Self-determination theory (SDT) states that people are more likely to adopt healthy behaviors or self-care activities when their basic psychological needs for autonomy, competence and relatedness are supported (Ryan \& Deci, 2000). The concepts of autonomous self-regulation and perceived competence are assumed to be important in initiating and maintaining diabetes self-care behaviors. SDT differentiates types of behavioral regulation in terms of the degree to which patients regulate their autonomously or selfdetermined as opposed to controlled regulation, which refers to experiences of pressure or coercion to behave in a certain way (Williams et al., 2009). Treatment self-regulation is concerned with the degree to which a person's motivation to follow a treatment regimen is autonomous. Patients who take their medication because they believe in its efficacy and are personally committed to improving their health are considered autonomous, whereas their behavior is considered to be controlled if they take their medication because their health care providers or social environment pressures them to do so (Williams, Freedman, et al., 1998). Perceived competence means that patients are capable and effective in achieving desired outcomes (Williams et al., 2009). Previous research has shown that people are more likely to feel competent to achieve important outcomes when they feel autonomous to make behavioral choices (Williams, Freedman, et al., 1998). In addition, increases in perceived autonomy and competence have been found to be related to improved glycemic control (Williams, Freedman, et al., 1998; Williams et al., 2004; Williams, Rodin, et al., 1998).

The quality of social contexts influences the motivation, performance and well-being of individuals who operate within them. In SDT, the concepts of autonomy support and control are used to characterize the quality of the health care environment. Autonomy support can be seen as an orientation towards patient-centered care among health care providers. Autonomy support facilitates patients' autonomous self-regulation and perceived competence for healthy behaviors (Williams et al., 2009). Previous studies have shown that patients report enhanced autonomy and competence in diabetes selfmanagement activities when they perceive their doctors to be more autonomysupportive (Ryan \& Deci, 2000; Williams, Freedman, et al., 1998; Williams et al., 2004; Williams et al., 2009)). Furthermore, clinicians' autonomy support has been found to correlate with psychosocial, physiological and biological outcomes regarding diabetes self-management (Williams et al., 2005) and quality of life (Williams et al., 2009). The current empirical base regarding the association of SDT concepts with diabetes outcomes is however mostly built on studies from the United States.

The aim of this study was to examine how Dutch type 1 and type 2 diabetes patients' perceived autonomy support from their primary care provider, as well as their perceived competence and treatment self-regulation, are associated with their diabetes self-care activities (healthy diet, physical activity, monitoring blood glucose and medication use) and general diabetes control. 


\section{Methods}

\section{Design and procedure}

A cross-sectional questionnaire survey was conducted. Three main strategies were used to recruit diabetes patients for participation. The first strategy to recruit them was through their health care providers. In parallel with the present study, we conducted a questionnaire survey among health care professionals working in diabetes care, and they were requested to recruit patients for the current study by handing out post cards at their practices or hospitals, inviting patients to complete a web-based questionnaire. Secondly, patients were recruited by means of a request posted on the website of the professional organization for pharmacists, asking pharmacists to hand out the same post cards among patients in their pharmacies. Thirdly, patients were recruited through the website of a Dutch television exercise program called 'The Netherlands on the Move!television' broadcast by the Dutch public channel 'Omroep Max'. This program was designed for adults and mainly targets people aged 55 and older (Meis et al., 2012). Ethical approval for this study was not required under Dutch law (Central Committee on Research Involving Human Subjects).

\section{Measures}

Demographic characteristics that were assessed included gender, age, educational level, country of birth, height and weight. Diabetes-related characteristics that were measured included type of diabetes and duration of diagnosis.

The measures used to assess perceived autonomy support, perceived competence and treatment self-regulation were preselected subsets of validated questionnaires based on SDT (Williams, Freedman, et al., 1998; Williams et al., 2004). Perceived autonomy support from the primary caregiver was measured using 10 items of the Health Care Climate questionnaire (HCCQ) (Williams et al., 2004) on a 5-point Likert scale (1= totally disagree to $5=$ totally agree; $\alpha=0.96$;). One example item was 'I feel that my physician has provided me choices and options'. Perceived competence was assessed with two items based on the Perceived Competence Scale for diabetes (PCS) (Williams, Freedman, et al., 1998; Williams et al., 2004) on a 5-point Likert scale (1= totally disagree to 5= totally agree; $\alpha=0.77)$. The items were: 'I can handle my diabetes well' and 'I can carry out the recommendations of my health care provider well'. Treatment self-regulation for medication use and/or glucose monitoring was measured with five items of the TSRQ (Williams, Freedman, et al., 1998; Williams et al., 2004) on a 5-point Likert scale (1= totally disagree to $5=$ totally agree). Patients were presented with the stem 'I take my diabetes medication and/or monitor my glucose because'. They then rated preselected responses that assessed controlled reasons ( 2 items; 'I would feel guilty if I didn't do what my doctor said' and 'Other people would be mad at me if I didn't') $(\alpha=0.69)$ and 
autonomous reasons (3 items: 'I personally believe that controlling my diabetes will improve my health', 'I would feel bad about myself if I didn't' and 'It's exciting to try to keep my glucose in a healthy range') $(\alpha=0.65)$. Treatment self-regulation for diet and exercise was assessed with five items of the TSRQ (Williams, Freedman, et al., 1998; Williams et al., 2004) on a 5-point Likert scale (1= totally disagree to 5 =totally agree). Patients were presented with the stem 'The reason I follow my diet and exercise regularly is that'. They then rated the same preselected responses that assessed controlled reasons ( 2 items; $\alpha=0.65$ ) and autonomous reasons ( 3 items; $\alpha=0.65$ ) for medication use and/or glucose monitoring.

\section{Dependent variables}

Diabetes self-care activities were assessed using four items from the summary of the Diabetes Self Care Activities questionnaire (Toobert, 2000). Participants were asked on how many of the last seven days they had kept up a healthy diet, participated in at least 30 minutes of physical activity, monitored their blood glucose as often as recommended by the health care provider, and took their recommended diabetes medication (0-7 days). The individual items were included separately in further analyses, in view of the low internal consistency of the scale $(\alpha=0.43)$. General diabetes control was measured with three items on a 5 -point Likert scale ( $1=$ bad to $5=$ excellent; $\alpha=0.70$ ) asking participants for an evaluation of their ability to cope with diabetes and regulation of their diabetes.

\section{Statistical analyses}

Body mass index (BMI) in kilograms per square meter was calculated from self-reported weight $(\mathrm{kg})$ and height $(\mathrm{m})$ and divided into categories in accordance with the standard BMI classification developed by the World Health Organization (World Health Organization, 2011). Educational level was recoded into a categorical variable (low level [no education or primary or basic vocational education], medium level [secondary vocational school or high school] and high level [higher professional education or university education]), according to the definitions by Statistics Netherlands (Verweij, 2008).

Analyses were carried out using SPSS 20.0 (Windows, Version 20.0). Reliability analyses were conducted for all subscales. Bivariate correlations between the study variables were calculated. Strong correlations were defined as Pearson $r$ coefficients exceeding 0.5 . Coefficients between 0.3 and 0.5 were regarded as moderately strong and coefficients smaller than 0.3 were defined as weak (Cohen, 1988). Independent samples ttests were conducted to assess differences in the study variables between patients with type 1 and type 2 diabetes. Only significant results are reported in text. In order to test differences in correlates between type 1 and 2 diabetes patients, interaction terms with type of disease (i.e. correlate*diabetes type) were added to the regression equation for the total sample. In case of significant interaction, stratified regressions were run for 
type 1 and type 2 diabetics separately. A stepwise approach using the Enter method was used in the linear regression analyses, with diabetes self-care activities as dependent variables. In the first step, the association between perceived autonomy support and the dependent variable was assessed, while adjusting for background and diabetes-related characteristics. In the second step, the measures of perceived competence and treatment self-regulation were added to the model. Subsequently, similar models were run with perceived general diabetes control as the dependent variable, while adding the diabetes self-care activities in the third step. $P$ values $<0.05$ were considered to indicate statistical significance.

\section{Results}

The sample consisted of 143 patients with type 1 diabetes and 384 patients with type 2 diabetes. Almost half of the patients had been diagnosed between 2 and 10 years ago. Table 4.1 shows their demographic characteristics. The mean age of the participants was 59.2 (SD 15.7) years and $51.3 \%$ were female. Almost half were low-educated and the majority had been born in the Netherlands. The mean BMI of the participants was 28.3 (SD 6.2).

\section{Means and correlations}

Overall, participants perceived a high degree of autonomy support, perceived competence and autonomous self-regulation for medication use and/or glucose monitoring and for diet and exercise (table 4.2). Participants perceived lower levels of controlled self-regulation for medication use and/or glucose monitoring than for diet and exercise. Patients with diabetes type 1 had significantly lower scores on controlled self-regulation regarding medication and/or glucose monitoring than type 2 patients $(T=2.00, p<0.05)$. 
Table 4.1: Demographic characteristics of the participants ( $N=527)$

\begin{tabular}{|c|c|c|}
\hline & Mean (SD) & $\%$ \\
\hline Age & $59.2(15.8)$ & \\
\hline \multicolumn{3}{|l|}{ Gender } \\
\hline male & & 49.5 \\
\hline female & & 50.5 \\
\hline \multicolumn{3}{|l|}{ Education } \\
\hline no/low & & 42.3 \\
\hline medium & & 33.7 \\
\hline high & & 23.9 \\
\hline \multicolumn{3}{|l|}{ Ethnicity } \\
\hline native & & 94.6 \\
\hline non-native & & 5.4 \\
\hline BMI & $28.3(6.3)$ & \\
\hline \multicolumn{3}{|l|}{ Type of diabetes } \\
\hline type 1 & & 27.1 \\
\hline type 2 & & 72.9 \\
\hline \multicolumn{3}{|c|}{ Duration diagnosis } \\
\hline$<6$ months & & 4.2 \\
\hline 6-12 months & & 3.6 \\
\hline $1-2$ years & & 9.3 \\
\hline 2-10 years & & 44.9 \\
\hline$>10$ years & & 38.0 \\
\hline
\end{tabular}

With regard to diabetes self-care activities, participants who used some form of medication for their diabetes reported taking their medication on almost every day of the week (respectively 6.8 out of 7 days (93.9\% daily) for diabetes type 1 patients and 6.6 out of 7 days (93.6\% daily) for those with type 2 diabetes. Furthermore, patients with type 1 diabetes reported to test their blood glucose on an average of 5.9 out of 7 days a week and $76.5 \%$ checked this on a daily basis. Type 2 diabetics scored significantly lower on testing their blood glucose ( 3.0 out of 7 days a week, $T=10.91, p<0.001$ (not in table)). The activities of following a healthy diet and engaging in at least 30 minutes of physical activity a day were carried out on an average of almost five days a week in both patient groups and approximately one third of the participants carried out these activities every day.

Patients who felt more competent and autonomy supported, perceived more general control over their diabetes (table 4.2). Additionally, we found significant, but moderate to low correlations between perceived competence and all self-care activities, except for the correlation with physical activity in type 1 patients. Our study also showed significant, but moderate to low correlations between perceived autonomy support on the one hand and controlled and autonomous self-regulation on the other in type 2 diabetics. Similar associations were found between perceived competence and self-care activities in these patients. For patients with diabetes type 1 we only found moderate to low correlations between perceived competence and autonomous self-regulation and 
between perceived autonomy support on the one hand and controlled self-regulation for diet and autonomous self-regulation for medication and/or glucose monitoring on the other. Following a healthy diet, engaging in 30 minutes of physical activity and testing blood glucose were positively correlated with autonomous self-regulation for diet and exercise in type 2 diabetics and general diabetes control in both patient groups.

\section{Multivariate linear regression analyses}

Analyses including interaction terms showed several significant results (i.e. controlled treatment self-regulation for medication and/or glucose monitoring*diabetes type, controlled treatment self-regulation for diet and exercise* diabetes type, autonomous treatment self-regulation for medication and/or glucose monitoring*diabetes type), justifying the stratified approach to study of correlates in type 1 and type 2 diabetics. Table 4.3 shows the two-step linear regression analyses on diabetes self-care activities separately for patients with type 1 and type 2 diabetes. Female and highly educated type 1 diabetics followed a healthy diet significantly more often than males and lower educated type 1 patients $(\beta=0.52, p<0.05 ; \beta=0.74, p<0.05)$. In both groups, a higher perceived competence was associated with following a healthy diet on significantly more days a week. High educated patients with type 1 diabetes engaged significantly less often in 30 minutes of physical activity than those patients with a lower level of education $(\beta=-0.73, p<0.05)$. Type 2 diabetics with a higher $B M l$ engaged significantly less often in 30 minutes of physical activity a day compared to type 2 diabetics with relatively lower weight $(\beta=-0.31, p<0.01)$. When patients with type 1 diabetes perceived more autonomous self-regulation regarding their medication and/or glucose monitoring, they reported monitoring their blood glucose more often.

Table 4.3 also shows the three-step linear regression analysis on general diabetes control. The final model explained $58 \%$ of the total variance in general diabetes control in patients with type 1 diabetes and 62\% in type 2 patients. Perceived competence was the strongest positive correlate of general diabetes control in both patient groups. Perceived competence appears to mediate the influence of autonomy support, especially in type 1 diabetics, while in type 2 diabetics, autonomy support remains to have a statistically significant explanatory contribution in the final model. Following a healthy diet was significantly positively associated with general diabetes control in patients with type 2 diabetes. Furthermore, female patients with type 2 diabetes reported a significantly lower general diabetes control than male patients (respectively $\beta=-0.22, p<0.05$ ).

\section{Discussion}

The aim of this study was to examine how Dutch type 1 and type 2 diabetes patients' perceived autonomy support from their primary care provider, as well as their perceived 
competence and treatment self-regulation, are associated with their diabetes self-care activities (healthy diet, physical activity, monitoring blood glucose and medication use) and general diabetes control. Overall, participants felt competent, supported in their autonomy, and perceived to autonomously self-regulate their diabetes self-care. Patients' perceived autonomy support was positively associated with their perceived competence, which in turn was positively associated with their participation in diabetes selfcare activities. Previous studies among patients with type 1 and type 2 diabetes have shown that patients report enhanced competence in diabetes self-management activities when they perceive their doctors to be more autonomy-supportive (Williams et al., 2004; Williams, Rodin, et al., 1998). In addition, autonomy support has been found to be related to psychosocial and biological outcomes of diabetes self-management (Williams, Freedman, et al., 1998). Patients with diabetes type 1 had significantly lower scores on controlled self-regulation regarding medication and/or glucose monitoring than type 2 patients.

With regard to diabetes self-care activities, most participants reported taking their medication almost every day. No significant differences were found in characteristics of patients that complied with their medication regimen and patients who did not comply. Approximately one third followed a healthy diet every day, which is similar to the findings of previous research (Shigaki et al., 2010). Furthermore, almost one third engaged in at least 30 minutes of physical activity every day, which is higher than in previous research which found only $4 \%$ to be exercising daily (Shigaki et al., 2010). However, overestimation of physical activity is common among adults with diabetes (Janevic et al., 2012), so the real number of days in our study could be lower. No significant differences in diet and physical activity behavior were found between type 1 and type 2 diabetes patients. Finally, $76.5 \%$ of type 1 diabetics reported to test their blood glucose on a daily basis. Patients with type 2 diabetes scored significantly lower on testing their blood glucose (31.2\% daily). The results are somewhat higher than has been found in previous research (Shigaki et al., 2010).

Patients with a higher perceived competence followed a healthy diet on more days a week than patients with a lower perceived competence. A previous study among adults with diabetes also found self-efficacy, a concept closely related to perceived competence, to be positively associated with adherence to diet (Senecal et al., 2000). Patients who perceived more autonomous self-regulation regarding their medication intake and/or glucose monitoring, reported monitoring their blood glucose more often. The findings of the current study are consistent with those of Shigaki et al. (Shigaki et al., 2010), who found a positive association between autonomous motivation and blood glucose testing among American diabetes patients. Previous studies have also reported a positive association between autonomous motivation and success in maintaining a broad range of health-related behaviors (Williams, Gagné, et al., 2002; Williams, Gagne, et al., 2002; Williams et al., 1996). In contrast, we only found a significant association between the following a healthy diet and perceived general diabetes control among 
diabetes type 2 patients, and did not find an association between autonomous selfregulation and physical activity and medication intake for both patient groups.

Previous studies suggested that professionals who support patients' autonomous self-regulation regarding medication use may enhance patients' quality of life, facilitate medication adherence, and improve physiological outcomes (Ryan \& Deci, 2000; Williams, Freedman, et al., 1998; Williams et al., 2004; Williams et al., 2009). In the current study, however, perceived competence appears to mediate the influence of autonomy support, especially in type 1 diabetics, while in type 2 diabetics, autonomy support remains to have a statistically significant explanatory contribution in the final model. The results indicate that health care professionals do not completely manage to influence perceived competence in type 2 patients by providing them autonomy support in contrast to type 1 patients. This is in line with our expectations since diabetes type 1 is generally diagnosed on a relatively young age, so patients learn to live with their diabetes earlier in live, which is expected to be easier. Overall, type 2 patients are diagnosed on a later age, which probably makes it more difficult to adjust their lives to their chronic disease.

Perceived autonomy support was only strongly significantly correlated with general diabetes control in type 2 patients, while its association with self-care activities was weak and not significant in both patient groups. Some patients may prefer a directive approach, in which the doctor gives more advice and tells the patients what to do rather than a patient-centered autonomy supportive approach in which the doctor gives little information and asks the patient what he/she wants (Deci \& Ryan, 2012; Vansteenkiste et al., 2012). The possibility for practitioners to be able to tailor their counseling style to the patients' individual needs for directives and autonomy support could be useful (Deci \& Ryan, 2012).

Some strengths and limitations of the current study need to be addressed. Since our study sample was self-selected, it is plausible that the participants had greater affinity with diabetes than the population as a whole. This may be of influence on the generalizability of our study results. Furthermore, patients could only participate through an online questionnaire and recruitment was conducted through health care professionals and a TV program on exercise, which may have led to bias in the composition of our study population. Another limitation is that the results are based on self-reported data, which may have been subject to bias (e.g. through social desirability factors). Some scales we used had a relatively low internal consistency. Moreover, we were not able to include an objective outcome measure, such as $\mathrm{HbA1C}$, in our study. Strong points are that our study was theory-based and that it is one of the first to assess the relation between SDT concepts and self-care activities and general diabetes control in Dutch diabetes patients. Moreover, to our knowledge no previous studies assessed differences between diabetic type 1 and type 2 patients in self-management behaviors and its determinants. Furthermore, our study was conducted among a rapidly growing patient population, which emphasizes the need for this type of research. The study variables ex- 
plained a high percentage of the variance in self-care activities and general diabetes control, which indicates the applicability of SDT in examining diabetes-related outcomes.

Our results emphasize the need for autonomy-supportive health care professionals in diabetes care. Professionals can be trained to be autonomy-supportive, and this concept is related to person-centered approaches, such as motivational interviewing (MI). Autonomy support is central to the practice of MI (Deci \& Ryan, 2012; Markland et al., 2005), and previous research showed that patients were more likely to perceive much autonomy support when their physicians used reflective statements, one of the key elements of MI (Pollak et al., 2011; Resnicow \& McMaster, 2012). Previous research also showed that patient-centered approaches, and specifically $\mathrm{MI}$, had a positive effect on self-management, patient satisfaction and improved psychological and glycemic outcomes (Chen et al., 2012; Resnicow \& McMaster, 2012) and quality of life (Lundahl et al., 2013). On the other hand, some patients may prefer a directive approach rather than a patient-centered approach (Deci \& Ryan, 2012; Markland et al., 2005). Attention for patients' individual needs concerning autonomy-supportive counseling is therefore recommended in diabetes education.

The importance of perceived competence in relation to self-care activities and general diabetes control of patients with type 1 and type 2 diabetes is also underlined by our study. Improving patients' perceived competence seems beneficial for their self-care activities, especially with regard to following a healthy diet. Williams et al. found that increasing perceived competence leads to greater motivation for diabetes self-care and better glucose control (Williams, Freedman, et al., 1998; Williams et al., 2004). Further research into the relation between perceived competence and perceived autonomy support with self-management in diabetes patients using a longitudinal design with path analysis is recommended. In addition, research focusing on differences in the underlying mechanism and determinants of self-management and general diabetes control between type 1 and type 2 diabetics is needed. 


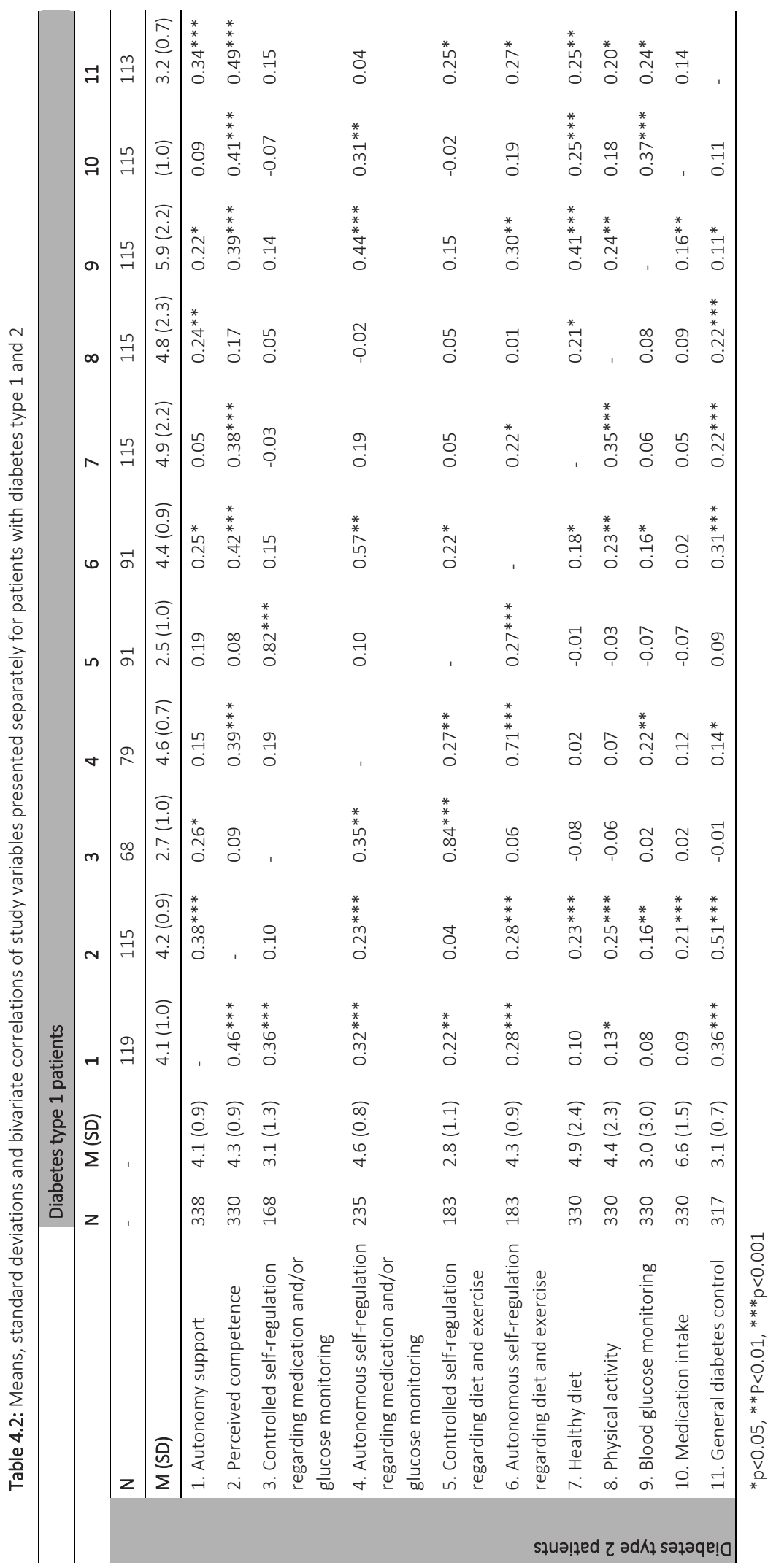




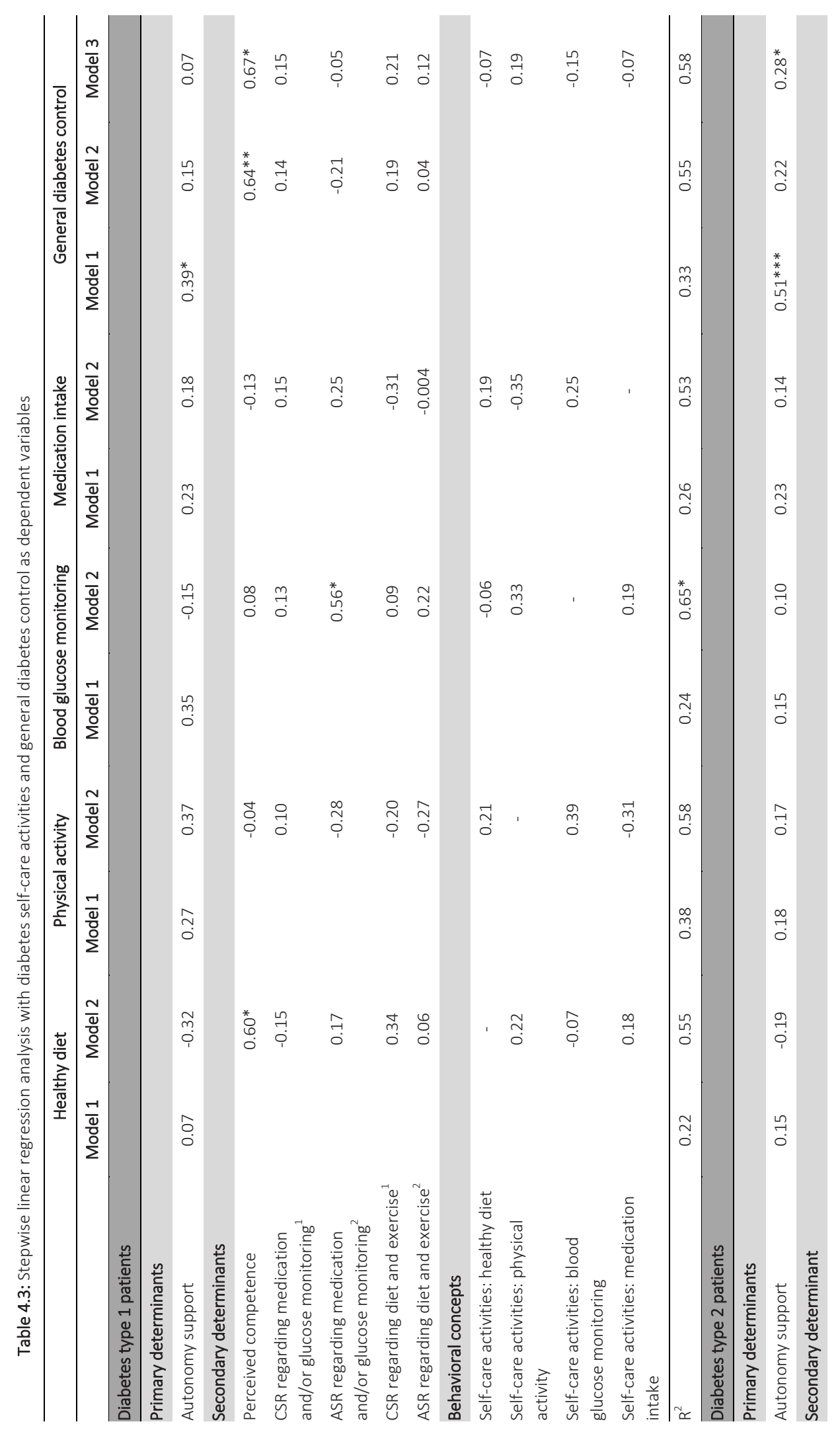




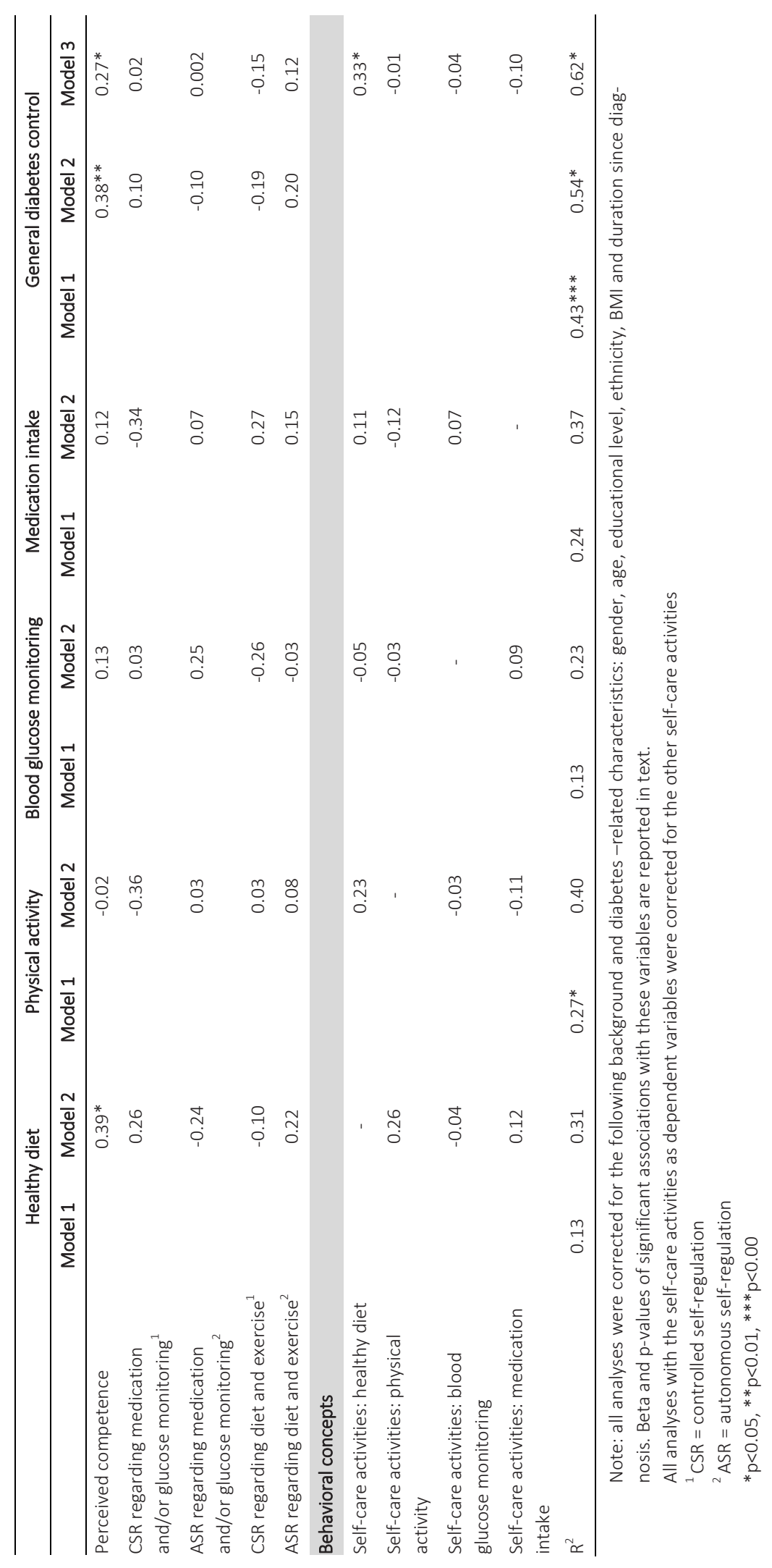





\section{Chapter 5}

\section{Mastery and perceived autonomy support are correlates of Dutch diabetes patients' self-management and quality of life}

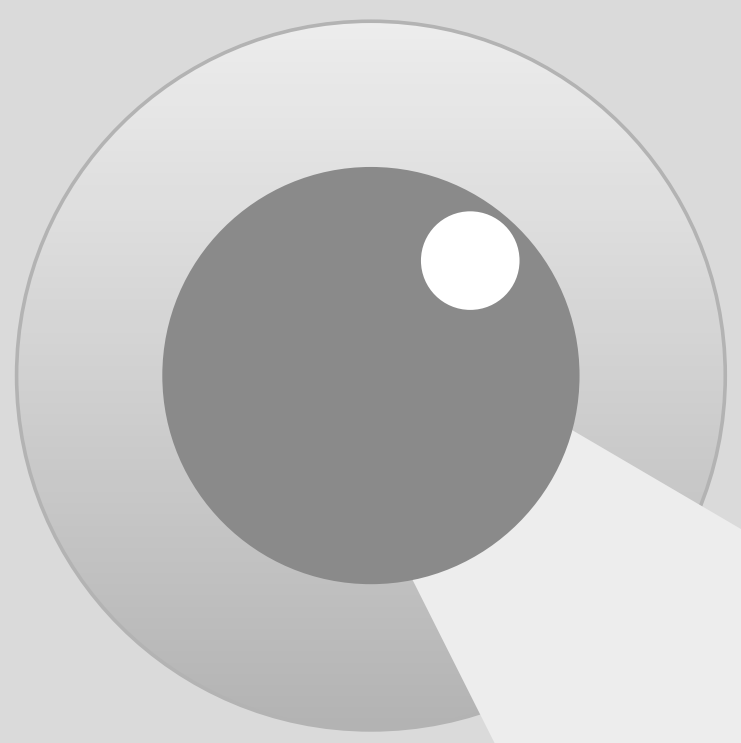

Published as: Raaijmakers, L.G.M., Martens, M.K., Hesselink, A.E., de Weerdt, I., de Vries, N.K., Kremers, S.P.J. Mastery and perceived autonomy support are correlates of Dutch diabetes patients' self-management and quality of life. Patient Education and Counseling, 97(1), 75-81. 


\section{Abstract}

Objective: The aim of this study was to assess the associations between type 2 diabetes patients' mastery and perceived autonomy support and their self-management skills and health-related quality of life (HRQOL).

Methods: A cross-sectional questionnaire survey was conducted among 3352 patients with type 2 diabetes. Key variables were assessed with validated questionnaires.

Results: Patients' mastery and perceived autonomy support correlated positively with their self-management skills $(r=0.34, p<0.001 ; r=0.37, p<0.001)$ and HRQOL $(r=0.37$, $p<0.001 ; r=0.15, p<0.001$ ). In the linear regression analysis, mastery and perceived autonomy support were positive correlates of self-management $(\beta=0.23 ; p<0.001 ; \beta=$ $0.25 ; p<0.001)$. Patients with more physical or psychological complications had significantly lower scores on mastery, perceived autonomy support, self-management and HRQOL.

Conclusion: Our results indicate the importance of mastery in relation to diabetes patients' perceived autonomy support, self-management skills and HRQOL.

Practice implications: Since a greater sense of mastery is likely to increase patients' autonomous motivation to cope with their disease, interventions can aim to influence patients' motivational regulation. In addition, we confirmed the need for autonomy support to improve patients' self-management skills. Professionals can be trained to be autonomy-supportive, which relates to person-centered approaches such as motivational interviewing (MI). 


\section{Introduction}

Diabetes mellitus is a rapidly growing health problem, which affects approximately 382 million people worldwide (International Diabetes Federation, 2013). The prevalence of diabetes in the Netherlands in 2011 was 801.000 and this number has been increasing by 87,000 a year (Baan et al., 2014). Diabetes is a complex chronic illness, since it affects various organs and systems and is often accompanied by other (chronic) diseases. In the Netherlands, the life expectancy of people with diabetes at the age of 45 is nine years less for men and eleven years less for women, compared to people without diabetes, and the corresponding numbers for diabetes patients at the age of 65 are four and six years less, respectively (Rijksinstituut voor Volksgezondheid en Milieu, 2007). People with diabetes have a better quality of life than people with most other serious chronic diseases (Rubin \& Peyrot, 1999). Moreover, diabetes patients without complications rate their quality of life only slightly below that of persons of similar age in the general population, but when complications appear, quality of life decreases considerably (Redekop et al., 2002; Rubin \& Peyrot, 1999). It is estimated that chronic complications occur in 40 to $56 \%$ of all people with type 2 diabetes (Rijksinstituut voor Volksgezondheid en Milieu, 2007). The overall goal for the treatment of diabetes is to prevent acute and chronic complications, while preserving a good quality of life. Knowledge about the occurrence of physical and psychological complications and its association with patients' selfmanagement and health-related quality of life ( $\mathrm{HRQOL})$ is therefore of great importance.

There is thus a need for effective treatment for diabetes, which includes continuous medical care and ongoing self-management by the patient and support to prevent complications (American Diabetes Association, 2011, 2013). Many aspects of the care for diabetes patients are nowadays managed by patients themselves on a life-long basis (Barlow et al., 2002; Reed et al., 2003). As a result, the responsibility for day-to-day disease management is shifting from health care professionals to the individual patients, which places a considerable burden on them (Barlow et al., 2002; Reed et al., 2003). Self-management refers to an individual's ability to manage the symptoms, treatment, physical and psychosocial consequences and life style changes inherent in living with a chronic condition (Barlow et al., 2002). The idea of self-management is based on the premise that people have the capacity to make choices and are responsible for the consequences of their choices (Feste \& Anderson, 1995). So far, mixed results have been reported on the association of self-management with biological outcomes and quality of life aspects in patients. Some previous studies have shown that better adherence to selfmanagement by diabetes patients can improve their glycemic control (Gao et al., 2013; Heisler et al., 2003; Norris et al., 2001)and quality of life(Cochran \& Conn, 2008). However, others have reported that engaging in self-management behaviors only has a minor effect on perceived health, quality of life and diabetes control (Maddigan et al., 2005; Watkins et al., 2000). Moreover, there have been few studies on the effectiveness 
of self-management in Dutch diabetes patients. It is hypothesized that self-management is associated with a higher HRQOL in type 2 diabetics.

Health care professionals aim to optimize self-management among their diabetes patients in addition to their responsibilities for periodical physical examination and checks on biomedical parameters, medication adjustments and provision of advice and support. In order to inform counseling and treatment, there is a need to identify primary determinants of self-management among diabetes patients. In Self Determination Theory the quality of the health care environment as regards the promotion of selfmanagement can be defined in terms of levels of autonomy support (Williams, Freedman, et al., 1998; Williams et al., 2004; Williams et al., 2009). Autonomy refers to acting in accordance with one's own values. Autonomy support in the health care setting can be seen as an orientation towards patient-centered care among health care providers, and it has been shown to facilitate patients' autonomous self-regulation and perceived competence in diabetes self-management (Ryan \& Deci, 2000; Williams, Freedman, et al., 1998; Williams et al., 2004; Williams et al., 2009). However, patients' self-care behaviors do not necessarily need to be valued behaviors (e.g. the need to take prescribed medication).

Furthermore, clinicians' autonomy support has been found to correlate with desirable psychosocial, physiological and biological outcomes (Williams et al., 2005) and quality of life (Williams et al., 2009). The current empirical base regarding the association perceived autonomy support with diabetes outcomes is mostly built on studies from the United States, where health is organized differently (Schoen et al., 2009). It remains unknown how this concept relate to self-management and HRQOL of type 2 diabetics in the Netherlands. Based on previous results, we expect perceived autonomy support to be positively associated with better self-management skills and a higher HRQOL in type 2 diabetics.

Another factor that appears to relate to self-management is personal mastery, a general sense of control over one's life and circumstances (Pearlin \& Schooler, 1978). A high level of mastery has been found to be associated with better health in people with chronic conditions or disability (Cott et al., 1999). From a broader Self Determination Theory (SDT) perspective the concept of mastery is closely related to the impersonal orientation of behavioral regulation (Deci \& Ryan, 1985). SDT proposes that people have dispositional causality orientations that refer to (a) the way people orient to the environment as regards information related to the initiation and regulation of behavior, and (b) the extent to which they are self-determined in general, across situations and domains(Deci \& Ryan, 2008). Individuals can be oriented by each of three general sources of behavioral regulation: autonomy, control and impersonal orientation. People with high scores for the impersonal orientation see themselves as incompetent and unable to master situations (Deci \& Ryan, 1985). Only a few previous studies have examined mastery or self-assessed control over life events in relation to psychological, behavioral or physical outcomes among diabetes patients (Mertens et al., 2012). These studies found 
that diabetes patients who reported a higher level of mastery had better scores for physical, mental and social functioning (Mertens et al., 2012), engaged in more recommended care practices and had higher scores for perceived health (Sloan et al., 2009). It is, however, unknown how mastery is associated with perceived autonomy support and HRQOL in Dutch diabetes patients. We hypothesize that patients with a lower sense of mastery have lower scores on perceived autonomy support and experience a lower HRQOL.

The aim of this study was to assess the associations between type 2 diabetes patients' mastery and perceived autonomy support and their self-management skills and HRQOL. The specific research questions addressed in this study were: 1) To what extent are patients experiencing complications due to their diabetes and suffering from one or more other chronic diseases? 2) What is the association between mastery and perceived autonomy support? 3) How are mastery and perceived autonomy support related to self-management and HRQOL in patients? 4) Are there differences in mastery, perceived autonomy support, self-management and HRQOL between subgroups (in terms of age, gender, educational level, BMI and the presence of other chronic diseases, physical and psychological complications)?

\section{Methods}

\section{Design and procedure}

A cross-sectional online questionnaire survey was conducted in collaboration with the Dutch patient association for people with diabetes. Several strategies were used to recruit diabetes patients for participation: (1) members of the diabetes patient association received an invitation for the online questionnaire by e-mail; (2) invitations were posted on the websites of several of the partner organizations of the patient association and disseminated via social media (e.g. Facebook and Twitter) (3) a call for participation was placed in the Dutch national newspaper De Telegraaf. Ethical approval for this study was not required under Dutch law (Central Committee on Research Involving Human Subjects).

\section{Measures}

The online questionnaire was based on questionnaires used in previous studies in the Netherlands (Mediquest, 2010; Raaijmakers et al., 2010) and was based on various existing validated instruments.

Background characteristics that were assessed included gender, age, educational level, country of birth, height and weight. Body mass index (BMI) in kilograms per square meter was calculated from self-reported weight $(\mathrm{kg})$ and height $(\mathrm{m})$. Educational level 
was recoded into a categorical variable (low level [no education or primary or basic vocational education], intermediate level [secondary vocational school or high school] and high level [higher professional education or university education]), according to the definitions used by Statistics Netherlands (Verweij, 2008). An additional category was added for participants who were still attending school.

The presence of other chronic diseases was assessed by asking participants to indicate whether they suffered from one or more other chronic diseases besides diabetes. Predefined answering options were provided (i.e. COPD, cardiovascular disease) as well as the possibility to enter options not listed. A sum score for the total number of chronic diseases was calculated.

Complications were measured with two items asking participants whether they had physical or psychological complaints. The items were formulated as follows: 'People with diabetes sometimes have physical or psychological complications. Do you have any physical complications and if so, which complications? Do you have psychological complications and if so, which complications?' Predefined answering options were provided for both items (i.e. high blood pressure, kidney problems, feet problems for physical complications and anxiety, gloominess and problems with sexual functioning for psychological complications), as well as the possibility to report complications other than those listed. Sum scores for the total number of physical and psychological complications were calculated.

Mastery was measured with a four-item version of the Pearlin Mastery Scale(Pearlin \& Schooler, 1978), including: 'I have little control over the things that happen to me', 'There is really no way I can solve some of the problems I have', 'There is little I can do to change many of the important things in my life' and 'I often feel helpless in dealing with the problems of life'. Answering options were presented as 5-point Likert scales ( $1=$ totally disagree; to $5=$ totally agree; $\alpha=0.83$ ). The use of a short version of the original Pearlin Mastery Scale is underpinned by a study assessing psychometric properties of a Swedish version of the Pearlin Mastery Scale (Mastery-S), which showed that two items of the original 7-item scale showed a misfit with the model (Eklund et al., 2012).

Perceived autonomy support from the primary caregiver was assessed using 10 items of the Health Care Climate questionnaire (HCCQ) (Williams, Freedman, et al., 1998; Williams et al., 2004) with 5-point Likert scales ( $1=$ totally disagree; to $5=$ totally agree; $\alpha=0.98$ ). An example of such an item is 'I feel that my physician has provided me with choices and options'.

Patients' self-management skills were assessed using four items with 5-point Likert scales ( $1=$ totally disagree; to $5=$ totally agree; $\alpha=0.67$ ) based on the PiH-NL questionnaire, a Dutch translation of the 'Partners in Health Scale' (Battersby et al., 2003). The items were: 'I am able to follow the recommendations of my health care provider', 'I can cope well with my diabetes', 'I always follow the prescriptions for my medication' and 'I stick to the life regimen I defined for myself'. 
Health-related quality of life (HRQOL) was assessed using the Euroqol 5-D instrument (Rabin \& Charro, 2001; The EuroQol Group, 1990). The Euroqol 5-D is a widely used and validated generic instrument that has five dimensions relating to 'mobility' (problems with walking), 'self-care' (problems with washing or dressing), 'usual activities' (problems with performing usual activities - e.g. work, study, housework, family or leisure activities), 'pain/discomfort' and 'anxiety/depression'. The response options are presented on a three-level ordinal scale ( $1=$ no problems, $2=$ moderate problems and $3=$ severe problems). The EQ-5D results in a five-digit code specifying a specific health state. An index score of quality of life was assigned to each self-reported health state. These index scores are based on a survey held among the general population, in which participants were asked to assign utilities to different self-reported health states. The EQ-5D index represents the valuation of the patient's health state from a general population's perspective, ranging from 0.549 to 1 , where 1 indicates perfect health, 0 indicates death and 0.549 indicates the worst possible health state that is viewed by the general public as considerably worse than death. A syntax based on the study by Lamers et al. (2005) in the Dutch population was used to assign index scores to the EQ-5D codes in our study (Lamers et al., 2005).

\section{Statistical analyses}

Analyses were carried out using SPSS 20.0. Reliability analyses were conducted for all subscales. To describe the study variables descriptive statistics were used. In order to assess the associations between the study variables, bivariate correlations were calculated. Strong correlations were defined as Pearson $r$ coefficients exceeding 0.5. Coefficients between 0.3 and 0.5 were regarded as moderately strong and coefficients smaller than 0.3 as weak (Cohen, 1988).

To assess the association of demographic characteristics and the presence of other chronic diseases, physical and psychological complications with mastery a multivariate linear regression analysis was conducted. To assess the association of demographic characteristics and the presence of other chronic diseases, physical and psychological complications and mastery with perceived autonomy support a stepwise approach using the Enter method was conducted in the linear regression analysis. The first model assessed the association of demographic characteristics, the presence of other chronic diseases, physical and psychological complications with the dependent variable. In the second model, mastery was added to the model. Subsequently, similar models were run with self-management skills and HRQOL as dependent variables, also including perceived autonomy support and self-management skills respectively as independent variables in the second model of the analyses. P-values $<0.05$ were considered to indicate statistical significance. 


\section{Results}

The sample consisted of 3352 patients with type 2 diabetes. Table 5.1 shows the demographic characteristics of the participants. Mean age of the participants was 64.1 (SD 9.3) years and $55.1 \%$ were male. These characteristics are comparable with the general population of type 2 diabetics in the Netherlands (Baan et al., 2014). Approximately one third were low-educated and the majority had been born in the Netherlands. Lower educated participants and ethnic minorities were somewhat underrepresented compared to the general diabetic type 2 population (Baan et al., 2014; Ujcic-Voortman et al., 2009). Mean BMI was 29.3 (SD 5.7) and $77.0 \%$ of the respondents were overweight or obese.

Table 5.1: Demographic characteristics of the participants ( $N=3352$ )

\begin{tabular}{lll}
\hline & Mean (SD) & $\%$ \\
\hline Age & $64.1(9.3)$ & \\
Gender & & \\
$\quad$ male & & 55.1 \\
$\quad$ female & 44.9 \\
Education & \\
$\quad$ no/low & 35.8 \\
$\quad$ intermediate & 30.7 \\
$\quad$ high & 33.5 \\
Ethnicity & \\
$\quad$ native Dutch & \\
$\quad$ ethnic minority & & 95.9 \\
BMI & & \\
\hline
\end{tabular}

Note: not all participants answered these questions, but drop-out was less than $1 \%$.

\section{Presence of other chronic diseases and complications}

A total of $57.1 \%$ of the participants reported suffering from one or more chronic diseases in addition to their diabetes (table 5.2). Most of these patients had one (52.6\%) or two (29.5\%) other chronic diseases. Furthermore, 93.5\% of the patients reported having physical complications and $35.2 \%$ had psychological complications. The number of physical complications was more or less evenly distributed over the categories 1 to 5 or more, and the majority of the participants with psychological complications reported one $(66.3 \%)$. 
Table 5.2: Presence of other chronic diseases besides diabetes type 2 and physical and psychological complications

\begin{tabular}{lllllllrrr}
\hline & $\mathrm{N}$ & $\%$ & Range & \multicolumn{5}{c}{ Number (\%) } \\
\cline { 2 - 9 } & & & & 1 & 2 & 3 & 4 & $\geq 5$ \\
\hline Chronic diseases & 3068 & 57.1 & $1-7$ & 52.6 & 29.5 & 11.0 & 4.7 & 2.2 \\
Physical complications & 3261 & 93.4 & $1-12$ & 14.1 & 19.9 & 20.9 & 17.3 & 27.8 \\
Psychological complications & 3261 & 35.2 & $1-6$ & 66.3 & 21.4 & 8.5 & 2.9 & 0.9 \\
\hline
\end{tabular}

\section{Means and correlations of self-regulation characteristics, self-management and HRQOL}

On average, participants perceived a high degree of autonomy support from their primary caregiver and rated their self-management skills as high. Patients perceived a relatively low sense of mastery and reported an average HRQOL score of 0.8 (SD 0.2) (table 5.3). Patients who perceived a high level of mastery had significantly higher scores for selfmanagement and HRQOL. Patients who felt supported in their autonomy had significantly higher scores for self-management skills. Furthermore, we found significant, but weak positive correlations between mastery and perceived autonomy support, between perceived autonomy support and HRQOL and between self-management and HRQOL.

Table 5.3: Mean scores, standard deviations and pearson correlations of study variables among type 2 diabetics

\begin{tabular}{|c|c|c|c|c|c|c|}
\hline & $\mathrm{N}$ & $M(S D)$ & Mastery & $\begin{array}{l}\text { Perceived } \\
\text { autonomy support }\end{array}$ & $\begin{array}{l}\text { Self- } \\
\text { management }\end{array}$ & HRQOL \\
\hline 1. Mastery (range 1-5) & 3031 & $3.7(0.9)$ & - & $0.19 * * *$ & $0.34 * * *$ & $0.37 * * *$ \\
\hline $\begin{array}{l}\text { 2. Perceived autonomy support } \\
\text { (range } 1-5 \text { ) }\end{array}$ & 3090 & $4.2(0.8)$ & & - & $0.37 * * *$ & $0.15 * * *$ \\
\hline $\begin{array}{l}\text { 3. Self-management } \\
\text { (range 1-5) }\end{array}$ & 3045 & $4.4(0.5)$ & & & - & $0.22 * * *$ \\
\hline 4. HRQOL (range $-0.33-1.00$ ) & 3284 & $0.8(0.2)$ & & & & - \\
\hline
\end{tabular}

${ }^{*} p<0.05, * * p<0.01, * * * p<0.001$

\section{Multivariate linear regression analyses}

Table 5.4 shows the linear regression analyses for mastery, perceived autonomy support, self-management and HRQOL. The linear regression analysis for mastery showed that the background- and diabetes-related characteristics explained $20 \%$ of the variance. Patients with a lower or intermediate level of education reported a significantly lower sense of mastery than higher educated patients. Patients suffering from more other chronic diseases, physical or psychological complications and patients with a higher BMI reported a lower sense of mastery.

The linear regression analysis for perceived autonomy support showed that the background- and diabetes-related characteristics and mastery explained only $7 \%$ of the 
variance. Patients with a higher sense of mastery perceived significantly more autonomy support from their primary caregiver. Younger patients and patients with a higher BMI had significantly lower scores for perceived autonomy support. Patients who reported more psychological complications perceived significantly less autonomy support.

Results from the linear regression analyses for self-management showed that adding mastery and perceived autonomy support to the second model increased the explained variance from $19 \%$ to $29 \%$. Mastery and perceived autonomy support were significantly positively correlated with patients' self-management skills. Younger participants and participants with a higher BMI reported lower self-management skills, as did patients who suffered from more physical and psychological complications.

The linear regression analyses for HRQOL showed that the variables in the final model explained $34 \%$ of the variance. Patients who had a higher sense of mastery rated their HRQOL significantly higher. Female participants and participants with a higher BMI rated their HRQOL significantly lower. Patients who suffered from more other chronic diseases, physical or psychological complications also rated their HRQOL significantly lower. 


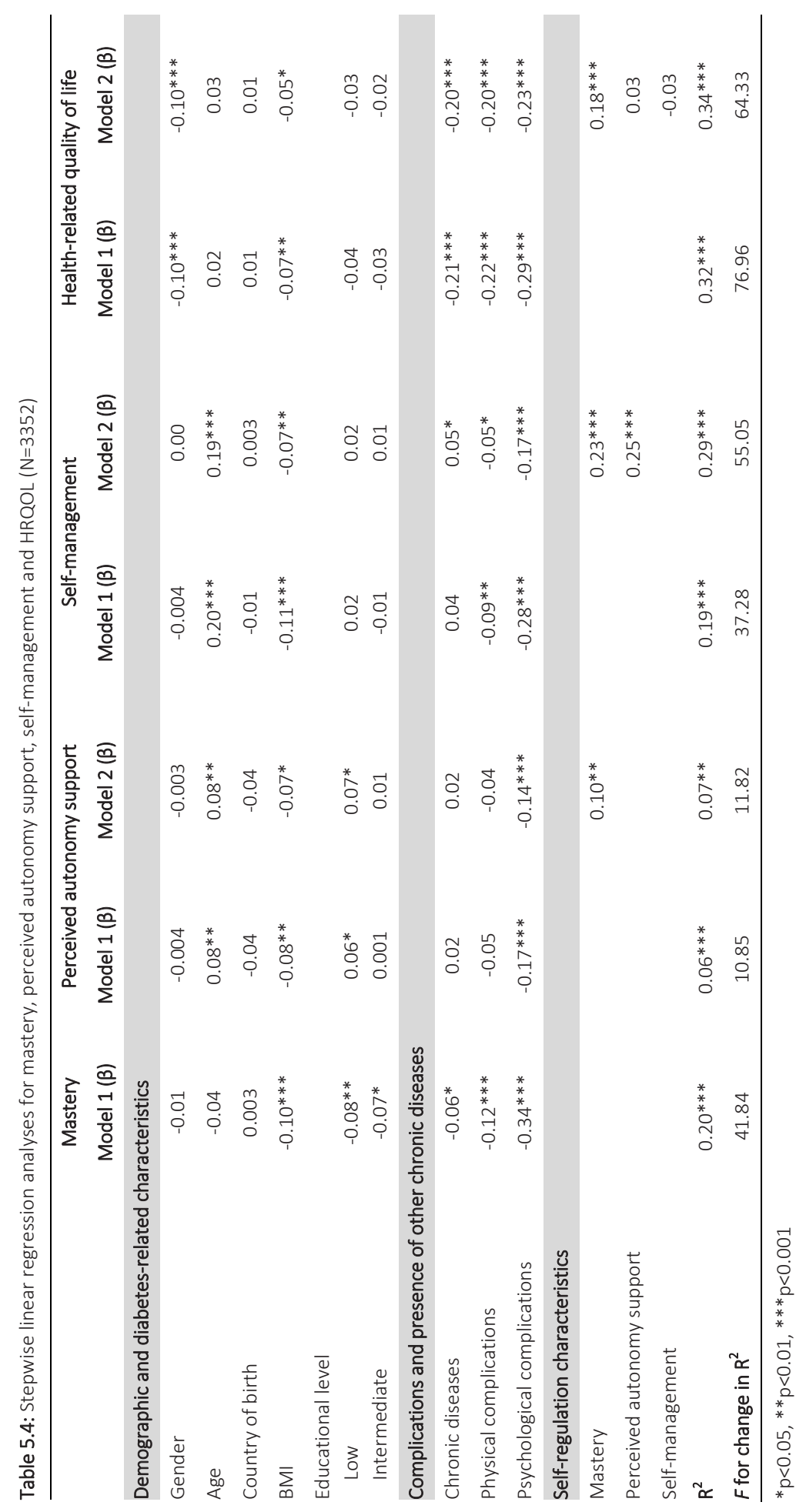




\section{Discussion and conclusion}

\section{Discussion}

The aim of our study among patients with diabetes type 2 was to assess the influence of their mastery and perceived autonomy support from their primary diabetes care provider on their self-management skills and HRQOL. Patients reported a relatively low sense of mastery. Patients with a higher sense of mastery perceived significantly more autonomy support and had significantly higher scores for self-management and HRQOL. Our results are in line with previous studies which showed that diabetes patients with a higher level of mastery had better scores for physical, mental and social functioning (Mertens et al., 2012) as well as higher scores for perceived health (Skaff et al., 2010; Sloan et al., 2009). Research has also shown that individuals with a high sense of mastery or control are more likely to use preventive care, seek treatment at an early stage, and use health services properly (Menec \& Chipperfield, 1997). In accordance with our results, previous research has shown that lower educated patients reported a lower sense of mastery than higher educated patients (Pearlin et al., 2007).

Consistent with previous studies, we found that patients who perceived more autonomy support from their primary caregiver had significantly better self-management skills (Williams, Freedman, et al., 1998; Williams et al., 2004). In contrast to previous research, which showed that autonomy support was strongly related to psychosocial and biological outcomes of diabetes self-management (Williams, Freedman, et al., 1998), we did not find a significant association between perceived autonomy support and HRQOL.

Self-management was correlated with $\mathrm{HRQOL}$, but the linear regression analysis did not reveal a significant association, indicating that the correlation between selfmanagement and HRQOL was confounded by the other study variables. Although previous research has shown that better adherence to self-management by diabetes patients can improve their quality of life(Cochran \& Conn, 2008), some other studies found that engaging in self-management behaviors only had a minor effect on quality of life (Maddigan et al., 2005; Watkins et al., 2000).

Participants reported an average EQ5D utility score of 0.82 (SD 0.2), which is comparable with previous research and only slightly lower than scores found in the general population (Redekop et al., 2002). The results of our study showed that patients' HRQOL was significantly lower when they suffered from more other chronic diseases and experienced more physical and psychological complications. This finding is in line with previous research, which showed that quality of life in diabetes patients seems to decrease particularly when complications appear (Glasgow et al., 1997; Redekop et al., 2002; Rubin \& Peyrot, 1999). Altogether, the variables in this study explained $34 \%$ of the variance in HRQOL. Further research should assess additional factors associated with HRQOL in diabetic patients, for example including other SDT concepts (such as relatedness/social support), preferably using a longitudinal study design. 
Some limitations of the current study need to be acknowledged. Since our study sample was self-selected, it is plausible that the participants had greater affinity with diabetes than the population as a whole, which may influence the generalizability of our study results. Furthermore, patients could only participate through an online questionnaire, which may have led to bias in the composition of our study population. Another limitation is that we did not use validated questionnaire to assess the study variables. Although these subsets are not validated as such, the scales show a Cronbach's $\alpha>0.7$, which indicates a high internal consistency for these scales in our sample. In addition, the results are based on self-reported data, which may have been subject to bias (e.g. through social desirability factors). Finally, the cross-sectional design excludes causal interpretations of the association between the study variables. A longitudinal design would be better in this respect. A strength of the current study is the large sample size. Furthermore, this study is one of the first to assess the relation between mastery and perceived autonomy support on the one hand and self-management and HRQOL on the other among Dutch diabetes patients. Moreover, our study was conducted among a rapidly growing patient population, which emphasizes the importance of this type of research.

\section{Conclusion}

Dutch type 2 diabetes patients were found to have relatively high scores for perceived autonomy support and self-management, and perceived a relatively low sense of mastery. This study provides concrete insights that can be used to inform intervention development with the purpose of improving self-management and HRQOL in type 2 diabetics. The findings emphasize and confirm the need for an autonomy-supportive counseling style by diabetes care providers to improve patients' self-management skills. Furthermore, our results underline the importance of mastery in relation to patients' perceived autonomy support from their primary caregiver, self-management skills and HRQOL.

\section{Practice implications}

Professionals can be trained to be autonomy-supportive using person-centered approaches, such as motivational interviewing (MI). Autonomy support is central to the practice of MI (Deci \& Ryan, 2012; Markland et al., 2005), and previous research showed that patients were more likely to perceive a high degree of autonomy support when their physicians used reflective statements, one of the key elements of MI (Pollak et al., 2011; Resnicow \& McMaster, 2012). Autonomy support can increase autonomy in patients, a prerequisite for self-management which enables them to be more selfdetermined and responsible. If patients are for example supported in autonomously engaging in a healthy diet by their health care professional, as a result of being more 
intrinsically motivated and doing because it is valued as personally important, this may lead to higher chance of maintenance of this self-management behavior (Williams, Freedman, et al., 1998). Furthermore, autonomy support is expected to facilitate patients' autonomous self-regulation and perceived competence for healthy behaviors (Williams, Freedman, et al., 1998). Another relevant autonomy supportive technique is shared decision making (Legare et al., 2010). This technique can be a safe starting point to increase patients' autonomy. To increase the adoption of shared-decision making, health care professional training may be important, as well as the implementation of patient mediated interventions such as decision aids (Legare et al., 2008).

Furthermore, we found mastery to be an important associate of patients' perceived autonomy support from their primary caregiver, as well as their self-management skills and HRQOL. According to SDT, mastery influences people's motivational regulation. A higher sense of mastery is assumed to increase patients' autonomous motivation. This influence on patients' motivational regulation and causality orientations can be a target for interventions that focus on aspects like changes in the counseling style of health care professionals. One option would be to tailor the counseling style to the patient's mastery level. Patients with a greater sense of mastery might recognize an autonomy-supportive counseling style more clearly or may even induce such a counseling style in health care professionals. On the other hand, patients with a lower sense of mastery are assumed to have a preference for a more directive counseling style. This is underlined by our finding that patients with a lower sense of mastery perceived less autonomy support from their primary caregiver. Health care professionals could readjust the personal goals of patients with a low sense of mastery. A previous intervention study also concluded that health professionals should take mastery perceptions of diabetes patients into account during regular consultations, with a view to improving treatment acceptance and adherence (Fall et al., 2013). Further research should identify risk groups based on personal characteristics, in order to recognize patients with low mastery levels and to tailor the counseling style to these patients. In addition, lifestyle interventions aimed at behavioral change and improving patients' skills and attribution style could be used to improve mastery. Referral to a psychologist for cognitive behavioral therapy (CBT) or integrating concepts of CBT in tailored self-management interventions could increase patients' sense of mastery over their life and control over their disease. Furthermore, elements of reattribution training could be integrated in self-management interventions as well, or in the counseling style used for patients with a low sense of mastery. Further research should however examine whether mastery can be seen as a dispositional personality trait or modifiable state, since previous studies have applied different assumptions (Deci \& Ryan, 1985; Schieman \& Turner, 1998). Moreover, the underlying mechanism of the association between mastery and self-management and HRQOL of diabetes patients should be assessed. 


\section{Chapter 6}

\section{The implementation of the Netherlands Diabetes Federation Care Standard for Diabetes anno 2013}

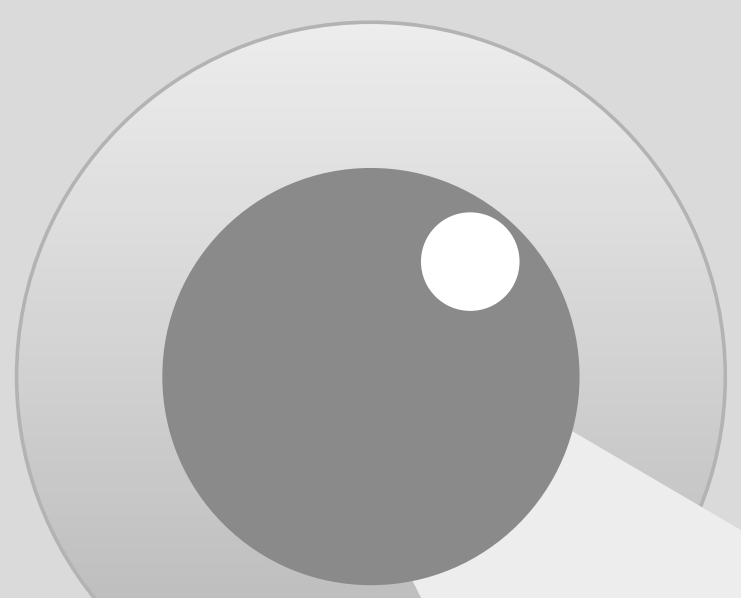

This chapter is based on the following publications (in Dutch):

Raaijmakers, L.G.M., Martens, M.K., Hesselink, A.E., de Weerdt, I., Kremers, S.P.J. (2013). De implementatie van de NDF Zorgstandaard Diabetes anno 2013. Nederlands tijdschrift voor Diabetologie, 11(3), 104-109.

Raaijmakers, L.G.M., Bagchus, C., Martens, M.K., Jonkers, R., Kremers, S.P.J. (2011). De implementatie van de NDF Zorgstandaard Diabetes: Onderzoek onder zorgprofessionals en patiënten in het kader van het Nationaal Actieprogramma Diabetes. Nederlands tijdschrift voor Diabetologie, 9(4), 167-174. 


\section{Abstract}

During the course of the Dutch National Action program Diabetes (NAD), two monitoring studies (2010 and 2013) were conducted among health care professionals and patients either with diabetes or at increased risk of developing diabetes. The main aim of these studies was to gain an overall view of the degree to which the Netherlands Diabetes Federation (NDF) Care Standard for diabetes (hereafter called Care Standard (CS)) was being implemented. This article describes the state of affairs in 2013 and compares the results of the study with those of the monitoring study of 2010.

Between November 2012 and February 2013 a cross-sectional questionnaire study was carried out among health care professionals $(n=2423)$ and patients $(n=5650)$. Between June and November 2010 a comparable study was carried out among 1726 health care professionals and 571 patients.

In 2013, significantly more health care professionals were in possession of the CS (43.7\%) compared to 2010 (37.6\%). The majority of the health care professionals (89.2\%) indicated that they worked largely or completely in accordance with the CS. In 2010, significantly fewer health care professionals had indicated that they worked largely or completely in accordance with the CS (79.0\%). Furthermore, the majority had a positive attitude towards the CS and its contribution to ensuring the quality of diabetes care in 2013. A high percentage of patients indicated that their health care providers involved them in their treatment, and reported that they were satisfied with the contact with their health care provider/s. Approximately $60 \%$ of the patients were familiar with the Zorgwijzer (the patients' version of the CS), and a small minority (15.3\%) was in possession of the Zorgwijzer. In 2010, more patients were unfamiliar with the Zorgwijzer $(50.0 \%)$, but the percentage of patients that had one in their possession was slightly higher (16.7\%).

Looking back at the NAD, it appears that the CS has provided momentum for the realization of various processes related to the improvement of the care for chronically ill people in the Netherlands. The next step could be to implement the care at a local level, and to embed it into a community approach thereby also focussing on the prevention of other chronic diseases. 


\section{Introduction}

In 2011 in the Netherlands 801,000 people were known to have diabetes and in the same year approximately another 87,000 new cases were diagnosed (Baan et al., 2014). There is a great need for good prevention and care. Over recent years there have been many initiatives aimed at improving the effectiveness and quality of diabetes care. One of these initiatives in the Netherlands, concerns the increased attention to continuity of care as a result of the initiative of the Dutch Ministry of Health, Welfare and Sport to start an integrated, programmatic approach of chronic diseases, such as diabetes (Ministerie van Volksgezondheid Welzijn en Sport, 2008). In order to deliver qualitatively good care in a multidisciplinary care setting, the Netherlands Diabetes Federation (NDF) has developed the Care Standard for diabetes type 2 hereafter called Care Standard (CS)). A Care Standard is a general framework outlining the services and treatment of people with a specific condition on a very aggregated level, while clinical guidelines describe the content of care in more detail, including how, when and by whom care should be provided (Coordinatieplatform Zorgstandaarden, 2010; Seidell et al., 2012). The CS describes the norm for generic multidisciplinary diabetes care and serves as a benchmark for the content, the organisation and the quality of programmatically structured diabetes care and prevention for people with diabetes (Nederlandse Diabetes Federatie, 2013b). The Dutch Diabetes Association (DVN) and the NDF have together produced a version of the CS (the Zorgwijzer) especially for patients with diabetes, which explains what a patient with diabetes may or can expect from their health care providers. The CS for type 2 diabetes does not apply to type 1 diabetes in adults and children. To fill this gap, a two-part addendum for type 1 diabetes is available, in which the care for adults and for children and adolescents with diabetes type 1 is described (Nederlandse Diabetes Federatie, 2013a).

In the view of the NDF, the care process requires up-to-date, multidisciplinary, evidence-based guidelines for the prevention, early detection and correct treatment of diabetes. These guidelines are integrated into the general management principles laid down by the CS (Nederlandse Diabetes Federatie, 2013b). The CS is regarded by the NDF as part of a greater whole which should serve to create the foundation of a national policy to help to limit the extent and severity of diabetes. To this end, the NDF has delivered the National Action program Diabetes (NAD) with funding of the Dutch Ministry of Health, Welfare and Sport (Nederlandse Diabetes Federatie, 2009). The overall purpose of the NAD was to create the circumstances, conditions and instruments necessary to slow down the increase in the number of people with diabetes and to reduce complications in diabetes patients. The main aim of NAD was the structural implementation of the NDF CS for diabetes. The NAD comprised a further five sub-themes, i.e. (1) prevention, (2) position of the patient \& client, (3) quality, organisation and knowledge, (4) regulation \& funding, and (5) e-communication and IC facilities. One of the specific objectives of the NAD was that by the time it ended that $90 \%$ of the health care profes- 
sionals must be familiar with the CS and that $50 \%$ of all health care professionals should regard it positively (Nederlandse Diabetes Federatie, 2009). An additional aim was that $90 \%$ of patients should be familiar with the Zorgwijzer, and that $50 \%$ of them should regard it positively (Nederlandse Diabetes Federatie, 2009).

In 2010 and 2013 two monitoring studies were carried out, the main aims of which were to gain insight into the extent to which the CS had been implemented in the setting of the NAD and the facilitators and barriers that played a role in this. Based on the main aims the following questions were formulated:

1. To what extent are health care professionals involved in this study familiar with the CS and working with it? And how do professionals appreciate the CS?

2. What are the facilitating and hindering factors that health care professionals experience in the care of diabetes, and more specifically in working in accordance with the CS?

3. To what extent have health care professionals experienced changes in diabetes care over the last 2.5 years?

4. Are people with diabetes and those at a high risk of developing diabetes, familiar with the Zorgwijzer and how do they appreciate it? To what extent do patients feel involved in their treatment?

5. What are the main differences with the results from the 2010 NAD monitor?

\section{Methods}

Between November 2012 and February 2013 a cross-sectional questionnaire study was carried out among health care professionals $(n=2423)$ and patients $(n=5650)$. Between June and November 2010 a comparable study was carried out among 1726 health care professionals and 571 patients (Raaijmakers et al., 2010). Both an online and a hard copy of the questionnaire were distributed to health care professionals. An online version only was completed by patients.

\section{Study in health care professionals}

Various recruitment strategies were used to recruit health care professionals. The main strategy was to send letters to all disciplines involved containing a link to a digital questionnaire. Also, a general appeal with a direct link to a digital questionnaire was disseminated via the NAD newsletter and via the websites of the journals of the professional organisations concerned. More information on recruitment strategies and an extensive report on the operationalising of the research methods can be found in the extended report about the NAD monitor of 2013 (Raaijmakers, Martens, Hesselink, et al., 2013).

As the CS is based on existing evidence-based guidelines (e.g. the Dutch College of General Practitioners' guideline), the questionnaires for each group of health care profes- 
sionals were formulated from a slightly different perspective. For the purposes of this article, five clusters of questions from the basic questionnaire were used: demographic characteristics, familiarity with and appreciation of the CS, working in accordance with the CS and changes in diabetes care.

To collect extra and more in-depth information in addition to the questionnaires, between November 2010 and January 2011, twenty-five health care professionals were interviewed. Thirteen interviews were carried out with health care professionals in primary care, eleven with professionals in secondary care and one interview was carried out with a health care professional working in public health. Participants included both key figures in Dutch diabetes care and 'ordinary' health care professionals. The interviews dealt with the same subjects as the questionnaire.

\section{Study in patients}

The study among patients was carried out in cooperation with the DVN. The main recruitment strategies were: an appeal to members of the DVN by e-mail, an appeal on the sites of various partners of DVN, and on social media. The online questionnaire was based on the questionnaires from the DVN 'Diabetes Zorgmonitor' (Mediquest, 2010) and the NAD monitor carried out in 2010 (Raaijmakers et al., 2010). Additionally, a number of questions were based on concepts from the Self-Determination Theory (Williams et al., 2004) and on the CS (Nederlandse Diabetes Federatie, 2013b). For this article, six clusters of questions were used. These questions concerned familiarity with and working in accordance with the Zorgwijzer, involvement in treatment, appreciation of care, health status and self-management. Before the questionnaire was launched online, a pre-test was carried out among patients, DVN experts and fellow researchers.

\section{Statistical analyses}

Data from the questionnaire were entered and analysed using SPSS 20.0. Descriptive statistics and frequencies were used to analyse the results. In order to make the comparison with 2010 reliable, the professions of podiatrist and chiropodist were not included in the analyses of the total group of respondents in 2013. These professional groups did not participate in the 2010 monitor, but accounted for a relatively large group in the 2013 monitor. In order to test the differences between various groups, independent sample t-tests, chi-squared tests and ANOVAs were carried out, depending on the outcome measure concerned. To compare the current results with those of the 2010 monitor, two sample t tests were used to test a selection of questions with average scores. In addition two proportion $z$ tests were carried out on a selection of questions with percentage scores. The results of these tests were only reported if they were statistically significant $(p<0.05)$. The rest of the comparisons with the results of the 2013 monitor are given descriptively. 


\section{Results}

\section{Results of the study in health care professionals}

In 2013 the questionnaire was completed by 2423 health care professionals and in 2010 by 1726 professionals. Table 6.1 gives an overview of the response to both studies divided into participating groups. The average age of the health care professionals in 2013 was 48.8 years (SD 9.0); in 2010 this was 46.6 years (SD 10.5). The majority of the participants were women (74.7\% in 2013: $73.3 \%$ in 2010). In 2013, 90.3\% of the health care professionals worked in primary care, $8.3 \%$ in secondary care, $0.7 \%$ in public health and $0.8 \%$ in home care. In 2010 these percentages were 68.5, 30.3 and 1.2, respectively.

Table 6.1: Response from health care professionals

\begin{tabular}{|c|c|c|c|c|}
\hline \multirow[t]{2}{*}{ Health care professionals } & \multicolumn{2}{|c|}{2013} & \multicolumn{2}{|c|}{2010} \\
\hline & $\mathrm{N}$ & $\%$ & $\mathrm{~N}$ & $\%$ \\
\hline General practitioners & 485 & 20.1 & 440 & 25.4 \\
\hline Practice nurses & 389 & 16.1 & 439 & 25.4 \\
\hline Diabetes nurses & 133 & 5.5 & 224 & 13.0 \\
\hline Primary care & 43 & & 86 & \\
\hline Secondary care & 74 & & 114 & \\
\hline Dieticians primary care & 135 & 5.6 & 163 & 9.4 \\
\hline Secondary care & 59 & & 90 & \\
\hline Home care services & 64 & & 68 & \\
\hline Physiotherapists & 8 & 0.3 & 142 & 8.2 \\
\hline Internal medicine physician & 26 & 1.1 & 67 & 3.9 \\
\hline Paediatricians & 36 & 1.6 & 61 & 3.5 \\
\hline Pharmacists / pharmacy assistants & 141 & 5.8 & 165 & 9.6 \\
\hline Podiatrist / chiropodist (diabetes qualified) & 1053 & 43.4 & & \\
\hline \multirow[t]{2}{*}{ Municipal Health Services staff* } & 17 & 0.7 & 25 & 1.4 \\
\hline & \multicolumn{2}{|c|}{$N=2423$} & \multicolumn{2}{|c|}{$N=1726$} \\
\hline
\end{tabular}

*Note: this question not put to these health care professionals, ${ }^{* *}$ These health care professionals did not participate in the 2010 study

Familiarity with the Care Standard

After exclusion of podiatrists and chiropodists, $43.7 \%$ of all health care professionals ( $n=$ 1367) were in possession of the CS. Additionally $21.9 \%$ were in possession of the addendum type 1 for adults and $32.7 \%$ reported they had the addendum type 1 for children and adolescents in possession. A total of $17.9 \%$ of health care professionals was unfamiliar with the CS, 42.8\% did not know about addendum type 1 for adults and $48.1 \%$ did not know about addendum type 1 for children. In comparison with the 2010 monitor, health care professionals were significantly more often in possession of the CS $(37.6 \%$; 
$\mathrm{p}<0.05)$. However, in 2010 health care professionals were significantly more often in possession of the addendum type 1 for adults $(34.5 \%, p<0.05)$ than in 2013 . Table 6.2 provides an overview of the degree of familiarity with the CS and addenda in 2010 and 2013 divided into professional groups.

Table 6.2: Possession of Care Standard and addenda per professional group

\begin{tabular}{|c|c|c|c|c|c|c|}
\hline \multirow{2}{*}{$\begin{array}{l}\text { Health care } \\
\text { professionals }\end{array}$} & \multicolumn{3}{|c|}{2013} & \multicolumn{3}{|c|}{2010} \\
\hline & $\begin{array}{l}\text { CS type } 2 \text { in } \\
\text { possession } \\
(\%)\end{array}$ & $\begin{array}{l}\text { CS in posses- } \\
\text { sion adden- } \\
\text { dum type } 1 \\
\text { adult (\%) }\end{array}$ & $\begin{array}{c}\text { CS adden- } \\
\text { dum type } 1 \\
\text { children \& } \\
\text { adolescents } \\
\text { in possession } \\
\text { (\%) }\end{array}$ & $\begin{array}{l}\text { CS type } 2 \text { in } \\
\text { possession } \\
(\%)\end{array}$ & $\begin{array}{l}\text { CS adden- } \\
\text { dum type } 1 \\
\text { adults in } \\
\text { possession } \\
\text { (\%) }\end{array}$ & $\begin{array}{l}\text { CS addendum } \\
\text { type } 1 \text { children } \\
\text { \& adolescents } \\
\text { in possession } \\
\text { (\%) }\end{array}$ \\
\hline General Practitioner & 34.9 & $*$ & $*$ & 40.4 & $*$ & $*$ \\
\hline Practice Nurse & 41.9 & 4.0 & 21.5 & 35.0 & $*$ & $*$ \\
\hline Diabetes nurse & 75.8 & 37.7 & 49.2 & 54.8 & 32.7 & 21.2 \\
\hline Dietician & 59.3 & 33.8 & 33.1 & 47.1 & 34.7 & 25.0 \\
\hline \multicolumn{7}{|l|}{ Physiotherapist } \\
\hline $\begin{array}{l}\text { Internal medicine } \\
\text { physician }\end{array}$ & 65.4 & 61.5 & 30.8 & 40.0 & 31.3 & 37.5 \\
\hline Paediatrician & 28.6 & 17.6 & 52.9 & 21.4 & 16.1 & 51.8 \\
\hline $\begin{array}{l}\text { Pharmacist / pharmacy } \\
\text { assistant }\end{array}$ & 36.9 & 28.9 & 38.5 & 23.9 & 14.4 & 11.0 \\
\hline Podiatrist / chiropodist & 24.3 & $*$ & $*$ & $* *$ & $* *$ & $* *$ \\
\hline $\begin{array}{l}\text { Municipal Health } \\
\text { Services staff* }\end{array}$ & 29.4 & $*$ & $*$ & 33.3 & $*$ & $*$ \\
\hline
\end{tabular}

\begin{tabular}{llllll}
\hline$N=1432$ & $N=830$ & $N=830$ & $N=2410$ & $N=759$ & $N=759$
\end{tabular}

*Note: this question not put to these health care professionals, ${ }^{* *}$ These health care professionals did not participate in the 2010 study

\section{Appreciation of the Care Standard}

A total of $67.7 \%$ of health care professionals indicated that they largely regarded the CS to be the norm for good diabetes care and $24.9 \%$ said that they regarded it to be completely the norm. In 2010, professionals significantly less often regarded the CS as being largely/completely the norm $(26.1 \%, 12.7 \%$; $p<0.05)$. Sixty-four percent regarded guidelines produced by their own professional organizations to be largely the norm for good diabetes care, and $31.3 \%$ regarded them as being completely the norm for good diabetes care. In 2010 guidelines produced by their own profession were significantly more often regarded as being largely or completely the norm $(57.8 \%, 26.0 \%, p<0.05)$. Of the respondents, $76.5 \%$ indicated that working in accordance with the CS was largely feasible and $10.9 \%$ found this to be completely feasible. In 2010 health care professionals 
significantly more often found working in accordance with the CS completely feasible (20.9\%), but significantly less often largely feasible (57.8\%). The majority of respondents (68.1\%) thought were the CS contributed greatly to ensuring the quality of care.

\section{Working in accordance with the Care Standard}

Health care professionals were asked to indicate on a scale of 1 to 5 the extent to which they think they work in accordance with the CS, $1=$ not at all and $5=$ completely. The majority of health care professionals (89.2\%) indicated that they worked largely or completely in accordance with the CS. Only $10.8 \%$ worked to a limited degree or not at all in accordance with the CS. In 2010, significantly fewer health care professionals had indicated that they worked largely or completely in accordance with the CS (79.0\%). In practice, the formulation of an individual care plan (ICP) and the collaboration within and between health care sectors were two aspects that were not yet being implemented in accordance with the CS. This is in line with the results of the 2010 monitor. A total of $28.5 \%$ of the health care professionals reported that they formulated ICPs. In 2010, $44.6 \%$ of health care professionals reported significantly more often that they formulated ICPs. Health care professionals in primary care worked in $52.3 \%$ of the cases structurally with other professionals in primary care. In secondary care this figure was $45.1 \%$ of the cases. In 2010, significantly more health care professionals in primary and secondary care reported that they worked structurally with other professionals in the same care sector (64.7\% and 59.3\%, respectively). In line with 2010, the annual check-up and accompanying examinations, the involvement of patients in their treatment, and taking part in professional education were implemented largely in accordance with the CS.

\section{Perceived barriers}

In 2010, 71.6\% of the health care professionals indicated to perceive barriers in diabetes care. The main barrier was the Lack of effective lifestyle interventions (or access to them) to provide care for people with diabetes (or at increased risk for it) (28.8\%). This barrier was confirmed in the interviews during which it emerged that the majority of the health care professionals regarded the availability of lifestyle interventions in their region - in particular exercise - as minimal, and/or were not aware of their existence. A quarter of the health care professionals had experienced barriers particularly in the care for groups that were difficult to reach, such as ethnic minorities and people with a low socio-economic position (SEP) (25.8\%). In the interviews it emerged that a lack of motivation and an unwillingness to take responsibility were mainly the problem in providing good care to these patients. The financial, legislative and regulations issues regarding care and prevention in accordance with the CS was also seen as an important barrier in implementing care and prevention (24.0\%). As in the questionnaire, the interviews revealed that almost all participating health care professionals clearly experienced the financing of diabetes care as a problem area. Another major barrier was the standardised recording and exchange of data (17.2\%). The interviews showed that this barrier 
was principally caused by the use of different registration systems in primary and secondary care. Health care professionals also had reservations about the registration and use of quality indicators in daily practice. Finally, working within multidisciplinary teams was also regarded as a barrier (14.6\%), particularly by dieticians and physiotherapists. The feeling that prevailed in these groups was that the role of their profession within diabetes care was not adequately recognised by other health care professionals or by the health insurers.

In 2013 the following five subjects were reported as being the most obstructive: the care of difficult-to-reach groups such as ethnic minorities and people with a low SES (41.8\%); financial aspects, legal and regulatory aspects of delivering care and prevention as defined by the CS (38.2\%); lack of effective lifestyle interventions (or access to them) to provide care for people with diabetes (or at increased risk for it) (34.0\%); standardised recording and exchange of data $(27.4 \%)$ and collaboration between primary and secondary care $(22.6 \%)$.

\section{Changes in diabetes care}

In 2013 health care professionals were asked to indicate on a scale of 1 to 5 if they thought that anything had changed in diabetes care over the past 2.5 years, $1=$ diabetes care has strongly deteriorated to $5=$ diabetes care has strongly improved. In $63.3 \%$ of the cases, health care professionals $(n=1048)$ indicated that diabetes care has improved, $17.1 \%$ thought it had strongly improved, $16.7 \%$ had experienced no changes, and $2.6 \%$ thought that it had deteriorated or strongly deteriorated (Table 6.3).

Table 6.3: Changes experienced in diabetes care over the last 2.5 years (reported 2013)

\begin{tabular}{lccc}
\hline Profession & $\begin{array}{c}\text { Improved, strongly } \\
\text { improved (\%) }\end{array}$ & Unchanged (\%) & $\begin{array}{c}\text { Deteriorated, strongly } \\
\text { deteriorated (\%) }\end{array}$ \\
\hline General practitioner & 81.3 & 17.2 & 1.5 \\
Practice nurse & 83.4 & 14.2 & 2.4 \\
Diabetes nurse & 79.4 & 16.7 & 3.9 \\
Dietician & 70.1 & 23.0 & 6.8 \\
Pharmacist / pharmacy assistant & 82.1 & 15.5 & 2.4 \\
Internal medicine physician & 72.0 & 24.0 & 4.0 \\
Paediatrician & 84.8 & 12.1 & 3.0 \\
Podiatrist / chiropodist & 83.4 & 18.6 & 19.5 \\
\hline N=1048 & & & \\
\hline
\end{tabular}

\section{Results of the study in patients}

In 2013, 5650 patients completed the questionnaire; in 2010, 573 patients completed it. Table 6.4 shows the background characteristics of the patients in both studies. In 2013, mean age of the respondents was 58.2 years (range 3-95); in 2010 this was 59.6 years 
(range 10-89). Just over half the patients were women and the majority had type 2 diabetes. In 2013, the questionnaire for respondents aged 12 years or younger was, in general, completed by the parents. In 2013, approximately one-third of respondents had a low level of education and the average body mass index (BMI) was 27.7 (SD 5.7); in 2010 this was 28.3 (SD 6.2).

\section{Familiarity with Diabetes Zorgwijzer}

In 2013, $15.3 \%$ of the patients were in possession of the Zorgwijzer and $40.4 \%$ was unfamiliar with it. In 2010 more respondents did not know about the Zorgwijzer (50.0\%), but also more respondents had the Zorgwijzer in their possession (16.7\%). The results of the current study showed that people with type 2 diabetes significantly more often had the Zorgwijzer in their possession than those with type 1 (19.2\% and $8.9 \%$, respectively). Additionally, those patients who were members of the DVN significantly more often had the Zorgwijzer in possession (17\%) than patients who were not members of the association (5.2\%). In line with 2010, patients were predominantly neutral about the Zorgwijzer as a useful tool in their diabetes care.

\section{Working in accordance with the Care Standard}

The majority of patients reported that they received an annual and a three-monthly check-up (88\% and $92.1 \%$ in $2013,87.2 \%$ and $85.0 \%$ in 2010 , respectively). A total of $22.6 \%$ of patients had an oral ICP, $6.2 \%$ had an oral and written ICP and $2.7 \%$ only had a written ICP. However, slightly more than half of the respondents (52.6\%) had no ICP and 15.9\% said they didn't know. In 2010, 40.0\% of the respondents reported they had an oral ICP, $17.1 \%$ had both an oral and a written ICP, and $34.6 \%$ had no ICP.

\section{Appreciation of care}

In general, patients were satisfied with their contact with their care provider/s and rated the health care climate positively. In line with 2010, in 2013 patients indicated that they were involved to a high degree in their treatment and, in general, they were satisfied with the care they received (Table 6.5). In 2013, patients who had the Zorgwijzer in possession and patients who were members of the DVN experienced a statistically significant higher degree of involvement in their treatment. 
Table 6.4: Background characteristics of patients

\begin{tabular}{|c|c|c|c|c|c|}
\hline & $\begin{array}{c}2013 \\
(\%)\end{array}$ & $\begin{array}{c}2010 \\
(\%)\end{array}$ & & $\begin{array}{c}2013 \\
(\%)\end{array}$ & $\begin{array}{c}2010 \\
(\%)\end{array}$ \\
\hline Sex & & & Country of birth & & \\
\hline Man & 48.8 & 48.6 & The Netherlands & 96.3 & 94.9 \\
\hline Woman & 51.2 & 51.4 & Surinam & 0.3 & 0.4 \\
\hline Age & & & Dutch Antilles / Aruba & 0.3 & 0.2 \\
\hline $10-20$ & 3.5 & 3.5 & Morocco & 0.0 & 0.7 \\
\hline $20-30$ & 3.3 & 4.0 & Indonesia & 1.0 & 1.6 \\
\hline $30-40$ & 4.9 & 4.8 & Other & 2.0 & 2.2 \\
\hline $40-50$ & 12.2 & 8.4 & & & \\
\hline $50-60$ & 23.2 & 17.4 & BMI & & \\
\hline $60-70$ & 35.9 & 40.7 & Underweight $(<18.5)$ & 0.8 & 1.5 \\
\hline $70-80$ & 14.9 & 18.7 & Normal weight (18.5-25) & 34.7 & 30.5 \\
\hline \multirow[t]{2}{*}{$>80$} & 2.1 & 2.6 & Moderately overweight (25-30) & 36.7 & 40.0 \\
\hline & & & Obesity & 27.8 & 29.1 \\
\hline Education & & & Type diabetes & & \\
\hline None & $*$ & 0.9 & Type 1 diabetes & 36.0 & 25.0 \\
\hline Scholar & 0.9 & $*$ & Type 2 diabetes & 59.4 & 67.3 \\
\hline Limited education & 32.6 & 42.0 & Another type of diabetes (MODY, & 2.4 & 1.2 \\
\hline Average education & 30.7 & 34.1 & LADA, gestational diabetes) & & \\
\hline \multirow[t]{2}{*}{ Higher education } & 35.8 & 23.0 & Raised risk of diabetes & 0.8 & 3.3 \\
\hline & & & Of unknown type & 1.5 & 3.2 \\
\hline Job situation & & & Treatment & & \\
\hline Paid work & 31.2 & 23.8 & No treatment & 0.8 & 2.4 \\
\hline Homemaker & 15.4 & 11.6 & Lifestyle advice & 1.7 & 26.9 \\
\hline Retired / Early retirement & 41.2 & 43.8 & Tablets & 50.5 & 54.5 \\
\hline School / Further education & 5.2 & 2.2 & Insulin & 69.0 & 54.7 \\
\hline Voluntary work & 9.5 & 2.4 & Blood pressure or anticoagulants & 54.4 & $*$ \\
\hline Unemployed / seeking work & 3.9 & 3.6 & Cholesterol-lowering medication & 56.1 & $*$ \\
\hline Invalid / Unfit for work & 10.2 & 9.8 & Other & 6.1 & 0.9 \\
\hline Self-employed & 5.1 & 2.9 & & & \\
\hline
\end{tabular}

* This answer category is not included in the questionnaire

Table 6.5: Involvement in treatment

\begin{tabular}{lcc}
\hline Involvement in treatment & $\begin{array}{c}2013 \\
\text { Average (SD) }\end{array}$ & $\begin{array}{c}2010 \\
\text { Average (SD) }\end{array}$ \\
\hline My care provider involves me enough in my treatment & $4.2(1.0)$ & $4.1(1.2)$ \\
I have the feeling that I can influence my treatment & $4.1(1.1)$ & $4.1(1.1)$ \\
I have the feeling that my care provider takes me seriously & $4.3(1.0)^{*}$ & $4.1(1.1)$ \\
\hline & $\mathrm{N}=5151$ & $\mathrm{~N}=525$ \\
\hline
\end{tabular}

${ }^{*} p<0.001$ 


\section{Discussion}

One of the specific objectives of the NAD was that by the time it ended that $90 \%$ of the health care professionals had be familiar with the CS and that 50\% of all health care professionals should have a positive attitude towards it (Nederlandse Diabetes Federatie, 2009). The majority of health care professionals (89.2\%) indicated that they worked largely or completely in accordance with the CS. This is a significant increase in relation to $76.1 \%$ in 2010, and indicates a positive trend in working in accordance with the CS. This is an important result, especially as recent research by the Dutch Health Care Inspectorate has shown that in 2012 the CS had still not been sufficiently implemented by the health care services (Inspectie van de Gezondheidszorg, 2012). The monitor also shows that the majority of the health care professionals are familiar with the CS (82.1\%) and that $43.7 \%$ are in possession of the CS. This is a significant increase when compared with the monitor of 2010. The objective of the NAD - that $90 \%$ of the health care professionals should know about the CS - has thus not yet been reached, but a positive trend is visible. The NAD objective that $50 \%$ of all health care professionals should have a positive attitude towards the CS has been reached. The majority of health care professionals in the random sample survey (89.2\%) indicated that they worked largely in accordance with the CS and viewed it and its contribution to the safeguarding of quality of care in diabetes positively. In comparison with the current monitor, in 2010 health care professionals significantly more often thought working in accordance with the CS was completely feasible. One explanation for this shift is that relatively more health care professionals now work fully or almost fully in accordance with the CS. As health care professionals are working with the CS, they are now also probably better able to judge if it is feasible to work with it. And, in addition, the chance that they will encounter hiatuses is bigger. In practice, the formulation of an ICP and collaboration within and between levels of care are two aspects that are not yet being carried out in full accordance with the CS. This is in line with the results from the monitor in 2010. It is therefore recommended that communication with the patients about the ICP should be more straightforward. It has been shown that the annual check-up and accompanying investigations, the involvement of patients in their treatment, and taking part in professional education were implemented largely in accordance with the CS. In 2010 also, these were the aspects that were also mostly carried out in accordance with the CS. The aim set by the NAD that $90 \%$ of people with diabetes or a high risk of diabetes should know about the Zorgwijzer, and that $50 \%$ should have a positive attitude towards it has not been reached. The results of the monitor show that only a small minority of patients (15.3\%) have the Zorgwijzer in their possession. On comparison with 2010 this is a slight drop; at that time 16.7\% said they were in possession of the Zorgwijzer. Between 2010 and 2012, familiarity with the Zorgwijzer rose from 50\% to 59.6\%. Patients were predominantly neutral about the Zorgwijzer as a useful tool, which is in line with the 2010 results. The results indicate that the level of familiarity with and the appreciation of the Zorgwijzer need to 
be further improved. In general, patients appear to be satisfied with the treatment they receive. A high percentage of patients indicated that their health care providers involved them in their treatment, and reported that they were satisfied with the contact with their health care providers.

\section{Implications for the future}

Looking back at the NAD, it appears that the CS has provided momentum for the realization of various processes related to the improvement of the care for chronically ill people in the Netherlands. As the CS for diabetes was the first Care Standard to be produced, experiences with the implementation of this standard can be used in the implementation of other Care Standards. The adoption stage of the CS has now been finished and the implementation stage has started. The implementation stage should be built upon in order to progress and make the transition to continuation and institutionalisation. Allowing the NAD or a follow-up programme to continue would be an asset, as in practice it would stimulate, facilitate and support each of the stages. The next step could be to implement the care at a local level, and to embed it in a community approach, thereby also focussing on the prevention of other chronic diseases.

\section{Strengths and limitations of the current study}

The results of the current study should be regarded in the light of a number of methodological considerations. The random sample survey is the result of self-selection so it is arguable that the health care and professionals in the survey have relatively more affinity and involvement with the CS and diabetes care than the general population. With respect to the comparison with the results of the 2010 monitor, it should be said that both studies were of a cross-sectional nature. This means that only a limited value can be attached to the significance of the differences found, taking into account that reliability within the group of participants cannot be guaranteed. The representativeness of the sample of health care professionals is indicated by the percentage of general practitioners working in a care group (78.0\%), which is in line with the estimated national percentage (Van Til, 2010). In 2010 this was 77.1\% (Raaijmakers et al., 2010). The representativeness of the patients is possibly less good taking into account that $36 \%$ of the respondents had type 1 diabetes and 60\% type 2 diabetes. This prevalence is not consistent with the national average where, since approximately $90 \%$ of all people with diabetes have type 2 diabetes (Baan et al., 2014). However other characteristics of the study population in 2013 are largely consistent with the composition of the study population in the 2010 NAD monitor (Raaijmakers et al., 2010). 
CHAPTER 6

\section{Table of Abbreviations}

NAD National Action program Diabetes

NDF Netherlands Diabetes Federation

CS Care Standard

ICP individual care plan 


\section{Chapter 7}

\section{The implementation of the National Action program Diabetes in the Netherlands: lessons learned}

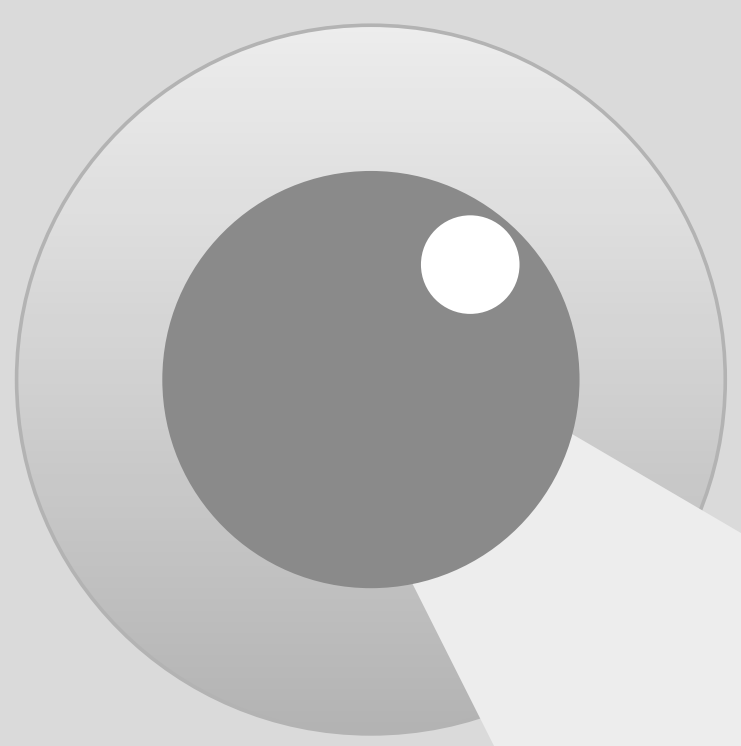

Submitted for publication as: Raaijmakers, L.G.M., Kremers, S.P.J., Schaper, N.C., de Weerdt, I., Martens, M.K., de Vries, N.K. The implementation of the National Action program Diabetes in the Netherlands: lessons learned. 


\section{Abstract}

Over the past decade, the National Action program Diabetes (NAD) was implemented in the Netherlands. Its aim was to introduce the Care Standard (CS) for diabetes by means of a specific implementation plan and piloting in several regions. This study aimed to provide insight into the implementation of the NAD as, coupled with the introduction of the CS, it may function as an example for similar approaches in other countries. A series of quantitative studies (participants 2010: $\mathrm{N}=1726$, participants 2013: $\mathrm{N}=1370$ \& participants pilot regions 2013: $\mathrm{N}=168$ ) and qualitative studies (participants 2010: $\mathrm{N}=18$ and participants 2013: $\mathrm{N}=4$ ) was conducted among health care professionals (HCPs). In addition, two quantitative studies were conducted among type 1 and 2 patients (participants 2010: $N=573$; participants 2013: $N=5056)$. Overall, positive changes in diabetes care were detected in the period 2010 - 2013. The CS has become more prominent and embedded in daily health care practice. A comparison of the results in specific pilot regions with the rest of the country revealed that HCPs in these regions scored significantly more positively on implementation and appreciation of the CS. This positive trend was reflected by the high levels of reported patient satisfaction and involvement in treatment. In retrospect the CS has provided momentum for the realization of various processes relating to the wider implementation of standards to improve the care for people with other chronic diseases in the Netherlands. Experiences with the NAD and CS underline the need to move towards an integrated multidisciplinary approach of diabetes care worldwide. 


\section{Introduction}

Diabetes is a multifactorial health problem that requires a multidisciplinary approach to prevention and treatment. As in most industrialized countries, Dutch health care faces the challenge of guaranteeing continuity and quality of care for the growing number of people with diabetes. For this reason multiple changes in diabetes care have been introduced in recent years. This study aimed to provide a broader perspective on these changes by integrating several studies conducted among health care professionals (HCPs) and diabetes patients.

A national integrated, programmatic approach to chronic diseases has laid the foundation for providing multidisciplinary care in a seamless manner in the Netherlands (Coordinatieplatform Zorgstandaarden, 2010; Ministerie van Volksgezondheid Welzijn en Sport, 2008; Struijs \& Baan, 2011). Two important elements of this approach are the Care Standard (CS) for diabetes developed by the Netherlands Diabetes Federation (NDF) and the bundled payment approach for financing (3). A CS differs from clinical guidelines, in that it is a general framework outlining the services and treatment to be delivered to people with a specific condition on an aggregate level; clinical guidelines on the other hand describe the content of medical care in more detail, including what, why and when care should be provided (Coordinatieplatform Zorgstandaarden, 2010; Nederlandse Diabetes Federatie, 2013d; Seidell et al., 2012). Furthermore, a CS is intended to provide HCPs, patients, researchers and funding bodies with a specification of the components of diabetes care, general treatment goals, and tools to evaluate the quality of care, also including paramedical treatment and prevention (American Diabetes Association, 2013). The Dutch Diabetes Association (DVN) and the NDF have together produced a version of the CS (the Zorgwijzer) especially for patients with diabetes, which explains what they can expect from their health care providers.

In the Netherlands, the CS functions as a general framework which overarches the guidelines of individual professional groups and focuses on a multidisciplinary approach to diabetes prevention and care. In addition, although not originally developed for this purpose, the CS is used as a purchasing instrument in the Dutch bundled payment approach for integrated chronic care (Struijs \& Baan, 2011). This approach has laid the foundation for delivering and funding diabetes care in accordance with the CS and has also partly led to the development of so-called care groups. Within this approach, insurers purchase the services and care described in the CS from a general contractor called a care group, which ends up in a bundled payment contract. These care groups are relatively new to the health care system and have been established to improve the quality of chronic care (Inspectie van de Gezondheidszorg, 2012). A care group consists of several health care providers that form a legal unit. Based on the bundled payment contract, the care group takes clinical and financial responsibility for all assigned patients in diabetes care and in turn subcontracts to individual care providers (e.g. general practitioner, die- 
tician, internist etc.) or delivers parts of the services in relation to the various components of diabetes care itself (Struijs \& Baan, 2011).

The Netherlands can be regarded as unique in the use of Care Standards (Seidell et al., 2012). The CS for diabetes was the first one to be developed by the NDF in April 2003 and the NDF took a leading role in the implementation of this CS (Coordinatieplatform Zorgstandaarden, 2010; Nederlandse Diabetes Federatie, 2003). The NDF unites associations of patients and of important HCPs. In 2007 the NDF started a project to update the CS, which was widely announced in professional journals and at conferences and through contacts with NDF members. In 2009, the NDF started the National Action program Diabetes (NAD) (2009-2013), the main objective of which was the systematic nationwide implementation of the Care Standard (Nederlandse Diabetes Federatie, 2009). The NAD was conducted with funding ( $€ 10$ million) from the Dutch Ministry of Health, Welfare and Sport (Nederlandse Diabetes Federatie, 2009). The NAD comprises five subthemes based on an inventory and analysis of problem areas in the field: 'Prevention', 'Position of the patient and client', 'Quality, organization and knowledge', 'Rules and funding' and 'E-communication and IT facilities'. These subthemes are, accidentally, in line with the concepts of the Expanded Chronic Care Model, a framework that can be used to optimize the provision of care for patients with chronic diseases, to decrease the burden of the disease and to prevent its occurrence through population based health promotion (Barr et al., 2003). The model also advocates integrated care, disease management and the use of evidence-based care guidelines (Raad voor de Volksgezondheid en Zorg, 2011).

The implementation of the CS and Zorgwijzer was supported by several national communication strategies and a regional implementation approach in five pilot regions ( Nationaal Actieprogramma Diabetes, 2011). The national strategies that facilitated the implementation of the CS and Zorgwijzer were aimed at knowledge, attitude and behavior of various target groups (i.e. patients, HCPs and government, health insurers and employers) (Nationaal Actieprogramma Diabetes, 2011). The main objectives were to inform HCPs and patients about the existence of the CS and its associated benefit, and to make the CS easily accessible online. In addition, the implementation of the CS was facilitated through the development of more than 50 products which were related to the NAD themes, based on existing barriers in practice. Examples of these products include the 'National Transmural Appointment (LTA)' guideline and the 'E-diabetes set'. The NDF selected five pilot regions in the Netherlands to initially implement several of these products with the objective of improving them and then implementing them nationally in the future (Nationaal Actieprogramma Diabetes, 2011).

While the NAD was running, a series of quantitative and qualitative studies was conducted among large samples of Dutch diabetes care professionals and diabetes patients (Martens et al., 2013b; Raaijmakers, Martens, Bagchus, et al., 2013; Raaijmakers, Hamers, et al., 2013; Raaijmakers, Hesselink, et al., 2013). The aim of the present paper is to place this series of studies in a broader perspective in order to provide insight into 
the implementation of the NAD, which besides its evaluative function may also provide an example for similar approaches in other countries.

\section{Methods}

In 2010, two cross-sectional studies were performed, in which questionnaires were distributed among all HCPs involved in diabetes care (i.e. general practitioners, practice nurses, diabetes nurses, dieticians, physiotherapists, internal medicine physicians and pediatricians; $\mathrm{N}=1726)$ and type 1 and type 2 diabetes patients $(\mathrm{N}=573)$ (Raaijmakers, Martens, Bagchus, et al., 2013; Raaijmakers et al., 2010; Raaijmakers, Hesselink, et al., 2013). In 2013 a similar questionnaire-based monitoring study was conducted among 1370 HCPs and 5056 type 1 and 2 diabetes patients (Raaijmakers, Martens, Hesselink, et al., 2013; Raaijmakers, Hesselink, et al., 2013).

In addition, 18 semi-structured interviews with HCPs working in primary and secondary care (i.e. general practitioners, practice nurses, diabetes nurses, dieticians, physiotherapists, internal medicine physicians and pharmacists) were held between November 2010 and January 2011 (Raaijmakers, Hamers, et al., 2013). Participants were randomly selected from the database of the quantitative study conducted among HCPs in 2010 (Raaijmakers et al., 2010; Raaijmakers, Hesselink, et al., 2013). In March and April 2014 , four semi-structured interviews were held with directors/managers of care groups or collaborations of health care centers.

The implementation process of the NAD products in the five pilot regions was evaluated by means of focus group interviews among HCPs and the region coordinator involved in the implementation of several NAD products in each region. In addition, one focus group interview was conducted with the NAD implementation team to assess success and failure factors related to the implementation of the NAD products and one with the developers of one of the core NAD products (i.e. e-diabetes set). In total seven focus group interviews were conducted. The questionnaire study among HCPs in 2013 was also conducted among HCPs in the pilot regions $(\mathrm{N}=168)$. For a detailed description of the recruitment, measurement instruments and statistical analyses of the separate studies we refer to the studies of Raaijmakers et al.(Raaijmakers et al., 2010; Raaijmakers, Martens, Hesselink, et al., 2013; Raaijmakers, Hesselink, et al., 2013) and Martens et al. (Martens et al., 2013a; Martens et al., 2013b). 


\section{Results}

\section{Parallel processes facilitating the implementation of the Care Standard}

Simultaneously with the development and implementation of the CS for diabetes, the organization of diabetes care in the Netherlands has undergone several other changes, which have also influenced diabetes care and should be discussed here to provide a complete picture. First, the update of the CS in 2007 coincided with the development of the previously described bundled payment approach (Struijs \& Baan, 2011). The purchase function of the CS within this approach is primarily used on the level of care groups and the CS has become embedded in the Dutch health care system as a result of this function. The CS serves as a financial incentive in the negotiations with health insurers, making these more structured since care has to be delivered and purchased in accordance with the norm. Second, the bundled payment approach has partly led to the development of so called care groups, as previously described. The CS is used for purposes related to the organization and quality of care. The care groups often appear to develop their own standards and guidelines, but these are typically based on the CS to make them more generalizable to the national norm. Third, over the past decade, vertical substitution of care has taken place, i.e. the transfer of tasks between care-providers with different levels of expertise (Vrijhoef et al., 2001). In many general practices the care for diabetes patients is delegated to specialized practice nurses or diabetes nurses who are supervised by the general practitioner. These trained nurses are specialized in care for chronic diseases such as diabetes and can play an important role in educating patients and encouraging overall adherence to treatment (Ubink-Veltmaat et al., 2005). Moreover, standardized diabetes care, delivered by a specialized diabetes nurse, has been shown to be a good alternative to standard care by an internal medicine physician or general practitioner, with comparable results after one year in terms of treatment goals, and even better results in terms of patient goals and cost-effectiveness (Houweling et al., 2009). However, a reported negative side effect of this transfer of care is that the expertise of general practitioners in diabetes care is decreasing (Raaijmakers, Hamers, et al., 2013).

\section{Implementation of the Care Standard}

Overall, positive changes in the implementation of the CS and diabetes care were detected between 2010 and 2013 (Raaijmakers, Martens, Hesselink, et al., 2013; Raaijmakers, Hesselink, et al., 2013). To illustrate this, in 2013 significantly more HCPs were familiar with the CS than in 2010 (table 7.1). Additionally, significantly more HCPs perceived themselves to be working largely or completely in accordance with the CS. Furthermore, in 2013 more HCPs considered the CS to be largely or completely the norm for high quality care than in 2010. We also found significant differences with regard to 
the implementation of specific elements of the CS. For example, in 2013 HCPs more often provided education and information about lifestyle to the majority of their patients and HCPs in secondary care reported more structural collaboration with HCPs in primary care. Also in 2013 HCPs significantly more often reported that they involved all patients in their treatment and registered quality indicators (Raaijmakers, Martens, Hesselink, et al., 2013; Raaijmakers, Hesselink, et al., 2013).

The positive trend among HCPs was also reflected by the results of the studies among patients. Overall, diabetes patients were satisfied with their contact with their caregiver(s) (mean scores $\geq 7.8$ (scale 1-10)) and perceived a high degree of involvement in their treatment (mean scores $\geq 4.1$ (scale 1-5)); no significant differences were detected in the 2010-2013 period. Patients' familiarity with the Zorgwijzer had significantly increased by 2013. However, the Individual Care Plan (i.e. an integral part of the CS which supports patients in their self-regulation and consists of mutual agreements between patients and their care givers about the goals of diabetes treatment) appeared to be used less often, since in 2010 significantly more patients had indicated that they had such a plan in possession than in 2013 (Raaijmakers, Martens, Hesselink, et al., 2013; Raaijmakers, Hesselink, et al., 2013).

Table 7.1: Significant differences in the implementation of the CS between 2010 and 2013

\begin{tabular}{lcc}
\hline & 2010 & 2013 \\
\hline Health care professionals & $\%$ & $\%$ \\
\hline In possession of the CS & 37.6 & 43.7 \\
Working largely or completely in accordance with CS & 79.0 & 89.2 \\
Regarding the CS largely or completely as norm for high quality care & 38.8 & 92.6 \\
Providing education and information about lifestyle to almost all or all patients & 34.8 & 56.9 \\
Structural collaboration of HCPs in secondary care with primary care & 24.2 & 32.9 \\
Involving all patients in their treatment & 54.0 & 71.7 \\
Register quality indicators & 72.4 & 84.2 \\
Patients & & 59.6 \\
Familiarity with the Zorgwijzer & 50.0 & 31.5 \\
In possession of an Individual Care Plan & 57.1 & \\
\hline
\end{tabular}

Note: all differences were significant on $p<0.05$ level

\section{Implementation of the CS in the NAD pilot regions}

The comparison of the results of the questionnaire study among HCPs in the pilot regions with the HCPs in the rest of the Netherlands revealed that HCPs in these regions scored significantly more positively on possession and appreciation of the CS than HCPS in the other regions (table 7.2) (Martens et al., 2013a; Martens et al., 2013b). HCPs in the pilot regions were significantly more often in possession of the CS, more often considered the CS to be the norm for high quality care and they more often worked com- 
pletely in accordance with the CS. HCPs working in the pilot regions perceived financial, legislative and regulation issues significantly less often as a barrier. Finally, HCPs in the pilot regions significantly more often provided information and education about lifestyle to almost all or all patients and more often thought diabetes care had strongly improved over the previous 2.5 years than the HCPs in the other regions (Martens et al., 2013a; Martens et al., 2013b).

Table 7.2: Significant differences between pilot regions and other Dutch regions

\begin{tabular}{lcc}
\hline & Pilot regions (\%) & Entire sample (\%) \\
\hline In possession of the CS & 51.9 & 43.7 \\
Working largely or completely in accordance with CS & 89.2 & 79.0 \\
Regarding the CS completely as norm for high quality care & 38.2 & 24.9 \\
$\begin{array}{l}\text { Experiencing barriers in relation to financial, legislative and regulations } \\
\text { issues regarding care and prevention in accordance with the CS }\end{array}$ & 28.8 & 39.6 \\
Providing education and information about lifestyle to almost all or all & 63.8 & 55.9 \\
patients & & 16.2 \\
Experiencing strong improvements in diabetes care past 2.5 years & 26.5 & \\
\hline
\end{tabular}

Note: all differences were significant on $p<0.05$ level

\section{Possession of the CS associated with better quality of care}

Possession of the CS was shown to be associated with better quality of care. Professionals who were in possession of the CS (2010: 37.6\% versus 2013: 43.7\%) had significantly better scores on the implementation of several elements of the CS than HCPs who were not in possession of the CS (table 7.3). In 2010 and 2013, HCPs in possession of the CS more often provided education and information about lifestyle to the majority of their patients; they significantly more often involved all patients in their treatment and scored higher on structural collaboration with(in) primary and secondary care. They also scored significantly higher on having a written treatment protocol in practice and on having put down responsibilities and competences in writing in their practice in 2010 and 2013 (Raaijmakers et al., 2010; Raaijmakers, Hesselink, et al., 2013). Additionally, in 2013 HCPs who were in possession of the CS scored significantly higher on the use of an Individual Care Plan, the registration of quality indicators and structural collaboration with public health (Raaijmakers, Martens, Hesselink, et al., 2013). 
Table 7.3: Significant differences among HCPs according to possession of the CS

\begin{tabular}{|c|c|c|c|c|}
\hline \multirow[b]{2}{*}{2010} & \multicolumn{2}{|c|}{ Possessions CS } & \multicolumn{2}{|c|}{ Not in possession CS } \\
\hline & $\%$ & Mean & $\%$ & Mean \\
\hline $\begin{array}{l}\text { Providing education and information about lifestyle to almost } \\
\text { all or all patients }\end{array}$ & 37.5 & & 33.4 & \\
\hline Involving all patients in their treatment & 58.5 & & 51.1 & \\
\hline In possession of a written treatment protocol & & 4.3(range 1-5) & & 3.5(range $1-5$ ) \\
\hline Having put down responsibilities and competences in writing & & 4.1(range $1-5)$ & & 3.2(range $1-5$ ) \\
\hline Structural collaboration with(in) primary care & 63.2 & & 49.7 & \\
\hline Structural collaboration with(in) secondary care & 30.9 & & 23.6 & \\
\hline \multicolumn{5}{|l|}{2013} \\
\hline $\begin{array}{l}\text { Providing education and information to almost all or all } \\
\text { patients }\end{array}$ & 62.3 & & 51.2 & \\
\hline Involving all patients in their treatment & 74.8 & & 70.5 & \\
\hline Use of Individual Care Plan & 29.6 & & 21.1 & \\
\hline In possession of a written treatment protocol & & 3.8(range $1-5)$ & & 3.1(range $1-5)$ \\
\hline Having put down responsibilities and competences in writing & & 3.5(range $1-5$ ) & & 2.9(range $1-5$ ) \\
\hline Register quality indicators & 69.6 & & 42.5 & \\
\hline Structural collaboration with public health & 10.2 & & 5.5 & \\
\hline Structural collaboration with(in) primary care & 53.2 & & 38.6 & \\
\hline Structural collaboration with(in) secondary care & 35.0 & & 25.3 & \\
\hline
\end{tabular}

Note: all differences were significant on $p<0.05$ level

\section{Perceived facilitators and barriers in diabetes care}

The semi-structured interviews revealed several facilitators and barriers perceived by HCPs in diabetes care (Raaijmakers, Hamers, et al., 2013). One of the major facilitators was the more prominent role of the practice nurses and diabetes nurses in diabetes care, as a result of the substitution of care. Other reported facilitators were benchmarking and multidisciplinary collaboration, although there is still room for improvement in collaboration with certain professional groups (i.e. dieticians, physical therapists and pharmacists), and between primary and secondary care. The bundled payment system for the funding of diabetes care and the role of the health insurers were perceived as major barriers within the health care system. According to HCPs, the bundled payment system is not suitable for chronic diseases; it leads to higher costs and makes care less transparent. Furthermore, HCPs reported that the health insurers have a lot of influence, are dominant and collaboration with health insurers is very inflexible. Other important barriers reported by HCPs were the lack of motivation among patients and the lack of awareness among professionals of lifestyle programs and prevention initiatives for diabetes patients (Raaijmakers, Hamers, et al., 2013). 


\section{Discussion}

Before interpreting the results of our series of studies, some methodological considerations need to be made. The cross-sectional design of the questionnaire studies in 2010 and 2013 conducted among HCPs and patients excludes causal interpretations of the associations between the study variables. Furthermore, the results of our questionnaire studies are based on self-reported data, which may have led to bias, e.g. through factors related to social desirability. The strength of our studies was the use of a mixed methods design in which we conducted qualitative and quantitative studies at multiple stages of the implementation process, thus enabling triangulation. Moreover, we were able to provide insight into the implementation of the CS from the perspectives of both HCPS and patients, and we included large samples of patients and HCPs covering the full range of professions involved in diabetes care.

\section{NAD as best practice}

Looking back over the past decade, we can conclude that the focus on the implementation of the CS, through the NAD, has had positive results. Moreover, the experiences with the NAD have also provided us with a great deal of information necessary to take further steps in implementing Care Standards for other chronic diseases both in the Netherlands and elsewhere. Accompanying the implementation of the CS with a government funded NAD may have been an example of a best practice. Although the CS was available before the start of the NAD, little effort had been put in facilitating its implementation in practice. The NAD has given the CS the attention it needed to become widely known and used among professionals and has facilitated its easy accessibility. As a result of these efforts, the CS has become more prominent and embedded in daily health care practice. The findings of our studies largely support the approach of the NAD, since professionals' appreciation and use of the CS increased in the period 20102013. Moreover, the implementation of several elements of the CS significantly improved, with even more positive results in the NAD pilot regions. This indicates that in daily practice professionals have become more aware of the position and benefits of both the CS and the guidelines issued by their individual professional groups.

\section{CS as flywheel}

It is also clear that in the Netherlands the CS has provided momentum for the realization of various processes relating to the wider implementation of standards to improve the care for people with other chronic diseases. Multidisciplinary collaboration, registration, and use of quality indicators for the purpose of benchmarking, and substitution of care originally provided by family physicians and specialists to practice nurses and diabetes nurses, are a direct spin-off of the focus on and activities related to the implementation 
of the CS. The CS has created awareness of the importance of these aspects of care for chronic diseases. It seems plausible that these processes would also have come about without the focus on and efforts put into the implementation of the CS, but they would probably have taken much more time and effort. Moreover, the introduction of the Individual Care Plan and the increased focus on self-management means that the patient is now playing a more pivotal role in the treatment of their disease (Barlow et al., 2002; Reed et al., 2003). However, the question is whether changing the structure of care through the CS amongst others, also changes the care processes itself, since previous research on the effects of population-based disease management programs among Dutch care groups showed little impact of these programs on patients' health (Elissen et al., 2012).

\section{Lessons learned from the implementation of the CS}

Currently there are eight Care Standards covering diabetes, COPD, obesity, vascular risk management, cardiovascular diseases/TIA, hereditary breast- and ovarian cancer and Asthma among children and adults. Furthermore, two standards are under authorization, two are in development, six are planned and proposals for two more are being explored. The CS for diabetes was the first to be completed. Experiences with the implementation of this CS can be used to aid the adoption and implementation of Care Standards for other chronic diseases in the future. The currently finished standards are published, but their implementation is not accompanied by an approach such as the NAD and appears to receive less attention than the CS for diabetes. Yet, the CS for obesity and CVRM are available for use within the bundled payment approach.

The series of studies on the implementation of the CS has taught us several key lessons. They show that implementation takes time and effort. Developing and publishing a CS does not automatically mean that it will be used in practice. Partly due to the function of the CS as a purchasing instrument within the Dutch bundled payment approach for integrated chronic care, many policymakers assumed that the implementation would occur automatically. However, implementation means more than the adoption and diffusion of innovations (Greenhalgh et al., 2004; wRogers, 2012). Research into the implementation of innovations teaches us that successful implementation of interventions requires systematic planning during the development phase and that the interventions should made workable and integrated in everyday health care practice (Grol \& Wensing, 2006; May et al., 2007). However, in practice, the implementation of innovations often seems subordinate to the development of the product itself.

Organizations currently involved in developing the Care Standards for other chronic diseases could benefit from the existing knowledge and expertise related to the implementation of the CS for diabetes and the facilitating approach used in the NAD pilot regions. Previous research into the dissemination and implementation of quality interventions, i.e. quality improvement efforts, has recognized the tendency of innovators to 
re-invent the wheel instead of efficiently making use of existing knowledge and insights (Farquhar, Stryer, et al., 2002; Reeves et al., 2009). One explanation for this seeming reluctance to use existing expertise in the implementation of the CS is that organizations and professionals involved in the development of the Care Standards for other diseases were not involved in the implementation process of the CS for diabetes. As a result they did not obtain ownership of the CS and a low level of ownership is expected to hamper the implementation of innovations (Fleuren et al., 2004).

\section{Future directions}

Despite the multidisciplinary character of the care described in the CS, the CS itself is disease-specific, while multimorbidity is common among (elderly) diabetes patients (Luijks et al., 2012; Marengoni et al., 2011; Sinnige et al., 2013; Uijen \& van de Lisdonk, 2008). Together with our experiences with the implementation of the CS for diabetes this underlines the benefits that could be gained if diabetes care were to move towards an integrated multidisciplinary approach of chronic diseases worldwide. Although the etiology, management and prognosis of other chronic diseases are different, the organisation and quality of care need to meet the same criteria as the care for diabetes as disease management strategies are similar in most chronic diseases. Despite the international differences in health care systems, there is international consensus on the goals of treatment for diabetes and the same type and quality of care needs to be provided to patients with one or more chronic disease (American Diabetes Association, 2013). The Care Standards and the bundled payment approach are useful instruments in organising integrated care and stimulating multidisciplinary collaboration. Furthermore, preventive activities targeting chronic diseases need to become embedded in primary care and close collaboration with the public domain needs to be established within such an integrated multidisciplinary approach. In 2012, prevention was added to the CS by means of an addendum. The importance of the availability of prevention and care close to patients at a local level is underpinned by the positive results and experiences with the CS for diabetes in the NAD pilot regions (Martens et al., 2013a). Achieving local collaboration between prevention, care and other relevant parties involved is expected to be difficult and depends on several local preconditions, such as the intentions of current municipal administrators, embedment into existing policies, political support and funding (Martens et al., 2013a). However, the force of action in multiple environmental settings and levels, compounded by the collective ability to accelerate and strengthen each other's impact, can profoundly improve the nation's health (Institute of Medicine, 2012). Moreover, the community approach to chronic disease prevention has a high degree of generalizability, cost-effectiveness, ability to diffuse information successfully through use of community networks, and potential for influencing environmental, regulatory and institutional policies that shape health (Sasseville et al., 2012). 
Finally, the CS is currently mainly supply driven rather than tailored to the needs of patients (Elissen, 2013), while we see an international and national need to abandon the one-size-fits-all approach and move towards personalized care (Handelsman et al., 2011; Nederlands Huisartsen Genootschap, 2013). To meet this need in the Netherlands, a group of experts and scientists is currently - in collaboration with the NDF- working on the development of so called 'patient profiles', a new tool which can help HCPs to provide personalized care tailored to the needs of patients with diabetes. Within these profiles treatment-, personal- and environmental factors are taken into account when tailoring the care and treatment. The Netherlands is the first country worldwide that will start with evidence-based application of patient profiles (Rutten, 2014).

\section{Conclusion}

In general we can conclude that the CS is now firmly embedded in the Dutch health care system and the NAD has provided momentum for the realization of various facilitating processes relating to the implementation of standards to improve the care for people with a chronic disease in the Netherlands. The experiences with the implementation of the CS for diabetes underline the beneficial value of the ongoing process in which healthcare moves towards an integrated multidisciplinary approach of chronic diseases worldwide. Furthermore, organizations involved in the implementation of other Care Standards are advised to take time to embed these standards into daily practice and involve the professionals who use the CS in practice.

\section{Table of Abbreviations}

NDF Netherlands Diabetes Federation

CS Care Standard

HCP Health care professional

NAD National Action program Diabetes 



\section{Chapter 8}

General discussion

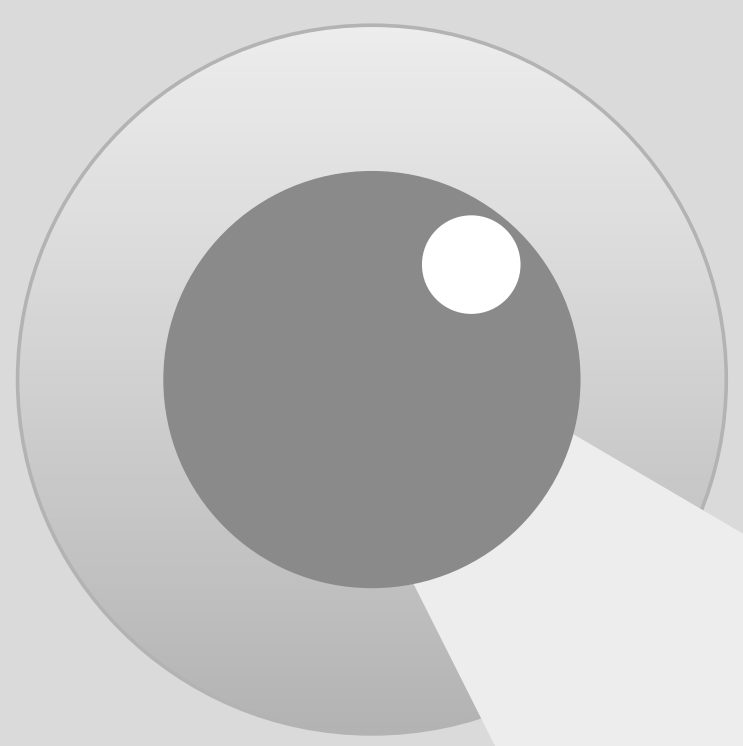


CHAPTER 8 
The aim of this dissertation was to provide a closer look at diabetes care and the introduction of the Netherlands Diabetes Federation (NDF) Care Standard (CS) for diabetes from the perspectives of both health care professionals and diabetes patients. From the perspective of health care professionals, this dissertation assessed their perceptions regarding the content and implementation of the CS and perceived facilitating and impeding factors in diabetes care. From the perspective of patients, this dissertation examined how Self-Determination Theory concepts and mastery were associated with Dutch type 1 and type 2 diabetes patients' self-management activities, general diabetes control and health-related quality of life.

This final chapter will provide an overview of the dissertation. First the main findings of the different studies will be summarized. Next, a reflection on the main results will be presented. Subsequently, strengths of this dissertation and methodological considerations will be outlined, followed by implications for practice. Furthermore, implications for theory and further research are discussed. This chapter ends with a general conclusion.

\section{Main findings}

\section{Perspective of health care professionals}

The cross-sectional questionnaire study reported on in chapter 2 assessed the perceptions of Dutch health care professionals ( $N=1547)$ regarding the $\mathrm{CS}$ and barriers to using it. A limited percentage of health care professionals had the CS in possession. Almost two-thirds of the professionals who were to some extent familiar with the CS thought it contributed greatly to ensuring the quality of care, and the feasibility of working in accordance with the CS was largely endorsed. However, professionals tended to perceive the guidelines issued by the own professional association as the norm for high quality diabetes care, rather than the CS. A minority of the professionals who were to some extent familiar with the CS described themselves as working in complete accordance with the CS. The main barrier to using the CS was the lack of effective lifestyle interventions (or access to them) to provide care for people with diabetes or those at increased risk for the disease.

In the qualitative study described in chapter 3, 18 health care professionals were interviewed in order to identify perceived facilitators and barriers in diabetes care using the Chronic Care Model (CCM). One of the major facilitators was the more prominent role of the practice nurses and diabetes nurses in diabetes care, as a result of the substitution of care. Other reported facilitators were the Cs itself and multidisciplinary collaboration, although there is still room for improvement in collaboration with certain professional groups (i.e. dieticians, physical therapists and pharmacists), and between primary and secondary care. Furthermore, benchmarking was perceived as a facilitating factor, 
although the majority reported the quality of the indicators for benchmarking to be a barrier. Major barriers in the health care system were the bundled payment system for the funding of diabetes care, the role of the health insurers and the lack of motivation on the part of patients. Another important barrier was the lack of awareness of lifestyle programs and prevention initiatives for diabetes patients among professionals, which indicated the usefulness of the Expanded CCM (Barr et al., 2003).

Altogether, professionals were positive about the CS, although only a minority had the CS in possession. Nevertheless, it is questionable whether possession of the CS is a necessary condition for working in accordance with the CS, especially since the majority reported working largely or completely in accordance with the CS. Organizational changes in diabetes care, as a result of the increased attention given to management of continuity of care, may have improved the implementation of the CS without health care professionals knowing about the CS or having it in possession. These changes have also brought about several barriers and professionals mainly perceived these in relation to the availability of prevention opportunities for lifestyle and the financing of diabetes care.

\section{Perspective of diabetes patients}

Chapter 4 describes a cross-sectional questionnaire study that examined how Dutch type $1(\mathrm{~N}=143)$ and type $2(\mathrm{~N}=384)$ diabetes patients' perceived autonomy support, as well as their perceived competence and treatment self-regulation, are associated with their diabetes self-care activities (healthy diet, physical activity, monitoring blood glucose, medication use) and general diabetes control. Most participants felt competent, supported in their autonomy, and had the perception of autonomously self-regulating their diabetes. The results underlined the importance of perceived competence in type 1 and 2 diabetics, as this was strongly associated with adhering to a healthy diet and general diabetes control. Our findings also emphasized the need for autonomy supportive health care professionals in diabetes care. However, perceived competence appeared to mediate the influence of autonomy support, especially in type 1 diabetics, while in type 2 diabetics' autonomy support remains to have a statistically significant explanatory contribution.

The cross sectional questionnaire study among 3352 patients with type 2 diabetes presented in Chapter 5 assessed the influence of diabetes patients' mastery and perceived autonomy support from their primary diabetes care provider on their selfmanagement skills and health-related quality of life (HRQOL). Dutch type 2 diabetes patients were found to have relatively high scores for perceived autonomy support and self-management, and to perceive a relatively low sense of mastery. Patients with more physical or psychological complications had significantly lower scores on mastery, perceived autonomy support, self-management and HRQOL, which is in line with our hypotheses. Our findings emphasized and confirmed the need for an autonomy-supportive 
counseling style by diabetes care providers. Furthermore, our results underlined the importance of mastery in relation to patients' perceived autonomy support from their primary caregiver, self-management skills and HRQOL and the lower level of mastery among patients with a higher BMI.

To recapitulate, the studies among diabetes patients show that patient's perceived autonomy support, perceived competence and mastery are important in relation to patients' self-management, general diabetes control en HRQOL.

\section{An integration of the perspectives of health care professionals and patients}

Chapter 6 describes two cross-sectional studies conducted in 2010 and 2013 among health care professionals and type 1 and type 2 diabetes patients. The main aim of these studies was to assess professionals' familiarity with and appreciation of the CS for diabetes and the quality of the implementation of the CS. Moreover, this chapter aimed to compare the results of both studies. In 2013, health care professionals were significantly more often familiar with the CS compared to 2010 and perceived themselves more often working completely in accordance with the CS. The majority had a positive attitude towards the CS and its contribution to ensuring the quality of diabetes care. Overall, diabetes patients were satisfied with their contact with their caregiver(s) and perceived a high degree of involvement in their treatment.

Chapter 7 aimed to provide insight in the implementation of the Netherlands Action program Diabetes (NAD) over the past decade, with the introduction of the Care Standard (CS) for diabetes as main aim, and including a specific implementation plan and piloting in several regions. This program and the introduction of the CS can function as an example for similar approaches in other countries. We conclude that the increased focus on the implementation of the CS has had positive results. In retrospect the CS has provided momentum for the realization of various processes relating to the implementation of standards to improve the care for people with a chronic disease in the Netherlands. Experiences with the NAD and the CS underline the need to move towards an integrated multidisciplinary approach of diabetes care worldwide.

\section{Reflection on main results}

This paragraph presents a reflection on the main results presented in this dissertation. First, the results in relation to the implementation of the CS and NAD will be discussed and then the function of the CS as flywheel will be reflected upon. 


\section{Lessons learned from the implementation of the CS for diabetes and the NAD}

Looking back over the past decade, we can conclude that the increased attention for the implementation of the CS, through the NAD, has had positive results. The CS for diabetes was the first one that was completed and experiences with the implementation of this CS can be used to inform the future adoption and implementation of Care Standards for other chronic diseases in the Netherlands and in other countries. Accompanying the implementation of the CS with a governmentally funded NAD may have served as a best practice. The CS was already available before the start of the NAD, but only minor effort was put in facilitating its implementation in practice. The active dissemination of the CS through the NAD has given the CS the attention it needed to become widely known and used among professionals and has facilitated its easy accessibility. Within this approach concrete communication strategies, activities and products have been developed to achieve the main objective, i.e. the nationwide implementation of the CS. As a result of these efforts, the CS has become more prominent and embedded in daily health care practice. Previous research concluded that passive dissemination of guidelines alone is not likely to adequately ensure appropriate uptake of recommendations in most circumstances (Fretheim et al., 2006). The findings of the studies we conducted largely support the approach of the NAD, since professionals' appreciation and use of the CS has increased in the period 2010-2013. This positive trend was reflected in the high levels of reported patient satisfaction and involvement in treatment. Moreover, professionals who were in possession of the CS had significantly better scores on the implementation of several elements of the CS than HCPs who were not in possession of the CS. The function of the CS as a general overarching framework seems clear to those who are familiar with it and the CS functions well as an addition to the guidelines of the individual professional groups. Altogether, the CS seems to serve as a supporting tool for professionals to organize diabetes care and it makes professionals and other relevant stakeholders aware of what high quality care means. However, based on the data of the studies presented in this dissertation we cannot conclude that the CS is truly necessary for delivery of high quality care or that the CS is a necessary addition to the guidelines of the individual professionals groups. Insight in the association of the implementation of the CS with more objective indicators of quality of care, for example the number of referrals of patients to secondary care, biomedical outcomes such as $\mathrm{HbA1c}$ and blood pressure in patients and lifestyle related indicators, such as smoking and BMI, could shed more light on these issues. Moreover, the CS is a prerequisite for applying the bundled payment approach in health care and the bundled payment contracts prescribe what care, as described in the CS, will be financed. The CS may therefore play a more important role on the level of care groups. 


\section{The CS as flywheel for chronic care}

In retrospect, the qualitative studies (see chapters 3 and 7) indicate that the introduction of the CS, supported by the NAD, and the bundled payment approach as a parallel development, have provided momentum for the realization of various processes relating to the implementation of standards to improve the care for people with a chronic disease in the Netherlands. It seems plausible that these processes would also have come about without the attention and efforts put in the implementation of the CS, but they would probably have taken much more time and effort. Multidisciplinary collaboration, registration and use of quality indicators for the purpose of benchmarking, substitution of care originally provided by family physicians and specialists to practice nurses and diabetes nurses and increased involvement of patients in their treatment are probably a direct spin-off of the attention for and activities related to the implementation of the CS and the NAD. The results of the studies among health care professionals in 2010 and 2013, reported in chapters 6 and 7, show an increase in collaboration and in registration of quality indicators. Moreover, the study described in chapter 3 shows that the role of the practice nurse and diabetes nurse was one of the major facilitators in diabetes care. In 2010 and 2013, professionals in possession of the CS significantly more often involved all patients in their treatment and scored higher on structural collaboration within and across primary and secondary care. In 2013, professionals in possession of the CS scored significantly higher on the registration of quality indicators and structural collaboration with public health (chapter 7). It seems that the CS created awareness of the importance of these aspects of care for chronic diseases. However, the question is whether changing the structure of care also changes the care processes itself, since previous research on the effects of population-based disease management programs among Dutch care groups showed little impact of these programs on patients' health (Elissen et al., 2012).

Chapter 3 showed that multidisciplinary collaboration was a major facilitator of diabetes care, since health care professionals perceived the collaboration both within primary care and between primary and secondary care to be effectively organized. In line with these results, De Bakker et al. (2012) showed that the bundled payment approach resulted within a relatively short period of time in the creation of care groups which provide integrated, multidisciplinary care for patients with diabetes (De Bakker et al., 2012). However, the results of chapter 3 showed that the collaboration between certain professional groups (i.e. dieticians, physical therapists and pharmacists) could be further improved, as could the collaboration between primary and secondary care (Raaijmakers, Hamers, et al., 2013). In 2011, one third of the dieticians in the Netherlands did not participate in disease management programs (Tol et al., 2013). An important disadvantage for the dietitian was that dietetic care was substituted by other disciplines, such as the practice nurse (Tol et al., 2013; Van Dijk \& Korevaar, 2011). Attention for this development is needed, since excluding dietitians from care groups may result in decreased access to dietetic care for patients within diabetes care groups, with limited freedom of choice as a result (Tol et al., 2013). 
We also found benchmarking to be a facilitating factor in diabetes care and this finding was underlined by previous research. Nobels et al. (2011) concluded that benchmarking is a promising tool for quality improvement in chronic care in general, and in diabetes care specifically, since feedback about professionals' clinical practice can lead to modifying their attitude and practice. However until 2011 there was a striking lack of clinical evidence from controlled trials (Nobels et al., 2011; O'Connor et al., 2009). A study describing Dutch policy developments to overcome the (financial) barriers to integrated care for chronic conditions in 2011 expected that quality of care would become more measurable and transparent not only for health insurers and care providers but also for patients as a result of the CS. This was expected to facilitate a better-informed choice of insurers and providers, which could contribute to improvement of the quality of chronic care (Tsiachristas et al., 2011). A recent prospective, randomized, controlled trial showed that benchmarking was an effective tool for increasing achievement of critical quality indicators (systolic blood pressure and LDL cholesterol) and potentially reducing patient cardiovascular residual risk profile among type 2 diabetics (Hermans et al., 2013). However, the review of Calsbeek et al. (2013) showed that there is a lot of variety in the content and development of sets of quality indicators used in studies on the quality of diabetes care in primary care settings. This reflects a lack of uniformity in the concept of diabetes care quality which hinders the interpretation of and comparison between quality assessments (Calsbeek et al., 2013).

Over the last decade, vertical substitution of care took place. This means that in many general practices the care for diabetes patients is delegated to practice nurses or diabetes nurses who are supervised by the general practitioner (Vrijhoef et al., 2001). These trained nurses are specialized in care for chronic diseases, such as diabetes and can play an important role in educating patients and encouraging overall adherence to treatment (Ubink-Veltmaat et al., 2005). Standardized diabetes care, delivered by a nurse specialized in diabetes appears to be a good alternative to standard care by an internal medicine physician, with comparable results after one year in terms of treatment goals, and even better results in terms of patient goals and cost-effectiveness (Houweling et al., 2009). The results of the study presented in Chapter 3 underlines, and showed that other health professions had confidence in the qualities of the specialized diabetes nurses and evaluated their contribution to diabetes care as highly positive. However, a reported negative side effect of this substitution is the possible decreasing expertise of GPs in diabetes care (Raaijmakers, Hamers, et al., 2013). In terms of the impact of physician-nurse substitution in primary care on clinical parameters, a systematic review and meta-analysis showed that trained nurses appeared to be better than physicians at lowering systolic blood pressure but similar at lowering diastolic blood pressure, total cholesterol or HbA1c. So far, there is however insufficient evidence that nurse-led care leads to better outcomes of other clinical parameters than physician-led care (Martinez-Gonzalez et al., 2014). Moreover, the National Transmural Appointment (LTA) guideline prescribes consultation of or referral to secondary care in the following 
cases: doubt about the diagnosis, problems with the glycemic regulation, problems with the treatment of risk factors, lack of control over complications in primary care and pregnancy (plans) (Sluiter et al., 2012).

In recent years, there has been an increasing recognition of patient-centered care as the best model to ensure a care respectful of, and responsive to patient preferences, needs, and values (Glasgow et al., 2008). The patient is now playing a more pivotal role in its treatment among others due to the introduction of the Individual Care Plan (as described in the CS) in the Netherlands and the increased importance of self-management. The Individual Care Plan, as integral part of the CS, supports patients in their selfregulation and consists of mutual agreements of patients and their care givers about the goals of their diabetes treatment. The results of the studies described in chapter 6 showed that patients experience a high degree of involvement in their treatment. However in 2013 approximately half of the patients did not have an Individual Care plan and formulating such a plan did not occur enough in accordance with the CS. A review on patient involvement in health care decision making showed that factors influencing patient participation consisted of factors associated with health care professionals such as doctor-patient relationship, recognition of patient's knowledge, allocation of sufficient time for participation, and also factors related to patients such as having knowledge, physical and cognitive ability, and emotional connections, beliefs, values and their experiences in relation to health services (Vahdat et al., 2014). Some of these factors may also play a role in the lack of correct use of an Individual Care or the use of such a plan at all. A Dutch study assessing the usability of the Individual Care Plan among health care professionals and diabetes patients showed that the Individual Care Plan does not fit the needs of the individual patient (Martens \& Hesselink, 2013). Another study among Dutch diabetes patients revealed that patients do not possess the motivation, knowledge and/or skills to take an active role in their treatment (Jansen \& Rijken, 2013).

\section{Strengths of this dissertation}

A distinctive strength of this dissertation is that diabetes care was assessed from the perspectives of both health care professionals and patients. In addition to assessing the implementation of the CS from the perspective of professionals, the studies conducted among patients provide in-depth knowledge about the consequences of the content and implementation of the CS on the level of patients in terms of the quality of care they (report to) receive, their ability to self-manage their disease and their health and healthrelated quality of life.

Furthermore, we conducted quantitative as well as qualitative studies and due to the use of mixed methods, triangulation of our data facilitates validation through cross verification from the different studies. Assessing diabetes care from both perspectives 
provided useful insights to inform health care and counseling in order to increase the quality of care for patients with diabetes.

The Netherlands can be regarded as unique in the use of the Netherlands Diabetes Federation (NDF) Care Standard (CS) for diabetes (Seidell et al., 2012). The studies presented in this dissertation are also exclusive in monitoring the nationwide implementation process. The results were fed back to the implementation institute, which used the results to adapt intervention contents and implementation strategies. In this respect, the present series of studies can be viewed as action research (Koshey et al., 2011). Action research, also known as Participatory Action Research (PAR), is an approach commonly used to improve conditions and practices in a range of health care environments (Koshey et al., 2011; Lingard et al., 2008). This type of research is characterized by involving professionals in order to help them improve practice. It includes action, evaluation, and critical reflection and - based on the evidence gathered through research - new objectives are formulated and changes in practice are then implemented. Action research is useful to improve practice, is participative and collaborative, situation-based and context specific. Action research can involve problem solving, if the solution to the problem leads to the improvement of practice (Koshey et al., 2011). In our studies, health care professionals and representatives of the NDF and of the NAD implementation team were involved from the start. The development of the measurement instruments occurred in collaboration with members of the NDF and pretests were conducted among health care professionals from all disciplines involved. In addition, a communication plan for the recruitment of professionals and patients was developed in collaboration with the NDF. Furthermore, preliminary results of the different measurements were presented at meetings with the NAD implementation team and board members and to employees of the NDF in order to support their objectives and actions in practice. In return, feedback from these representatives was used to further interpret the results and inform questions asked in qualitative studies. The results of the studies were also presented at conferences organized by the NAD that were attended by several types of professionals from practice (including health care professionals, researchers, and policy advisors).

Insight in perceptions of health care professionals and perceived facilitators and barriers in relation to the implementation of this Care Standard can inform future, similar approaches in other countries as well as the implementation of Care Standards for other (chronic) diseases in the Netherlands.

We included a large sample of health care professionals (Chapters 2, 6 and 7), consisting of a broad range of professions involved in diabetes care. The questionnaire studies among patients (Chapters 4, 5 and 6) were conducted among moderate size to large samples of a rapidly growing population, which emphasizes the relevance of this type of research.

Furthermore, by using the CCM to categorize the identified facilitators and barriers (chapter 3), we increased the standardization of reporting facilitators and barriers in relation to diabetes care. The results of the study described in chapter 3 show that ex- 
pansion toward better integration of prevention and health promotion, using the Expanded CCM, would be useful (Barr et al., 2003). Moreover, this dissertation indicates that expansion of the Expanded CCM could be valuable (also see implications for theory).

The use of semi-structured interviews (Chapters 3 and 7) provided the opportunity to collect in-depth information and understand perspectives and experiences of participants. Furthermore, all interviews were conducted by the same researcher, in order to increase consistency in the data collection process, and all codes were checked independently by two researchers to increase conformability (objectivity and neutrality).

The perspective of patients proved useful to incorporate the needs and wishes of patients in initiatives aimed to improve the quality of care. We used Self Determination Theory and related constructs to obtain insight in factors associated with selfmanagement and HRQOL in patients. Specifically, they were used to inform intervention development aimed at tailoring the provision of care and counseling style of health care professionals to the needs of patients. Moreover, self-reported HRQOL was an important outcome in our study. Previous research acknowledges the importance of this outcome, since clinical parameters, such as HbA1c often fail to capture the overall impact of diabetes (Maddigan et al., 2003).

\section{Methodological considerations}

The results of the different studies presented in this dissertation should be interpreted in the light of several methodological and practical limitations. Most shortcomings were already discussed in previous chapters. This section describes the most important considerations, which concern issues related to the study design, study population, measurement instruments and statistical analyses.

\section{Study design}

We used a cross-sectional design for the questionnaire studies conducted among health care professionals and diabetes patients. The primary limitation of a cross-sectional design is related to causality, since this design excludes causal interpretations of the associations between the study variables. Because the exposure and outcome are simultaneously assessed, there is no evidence of a temporal relationship between exposure and outcome. A longitudinal design would have been better in this respect.

\section{Study population}

Another limitation of the studies among health care professionals and patients is possible self-selection bias in the samples. Since participants were allowed to decide entirely 
for themselves whether or not they want to participate in the surveys, it is plausible that they had greater affinity with diabetes (care) than the population as a whole, which may have influenced the generalizability of our study results. Nevertheless, in some respects our samples of health care professionals were representative, e.g. the percentages of general practitioners working in a care group (77.1\% and $78.0 \%)$ were in line with the estimated national percentage (78.0\%) (Van Til, 2010). Moreover, all professions were represented in our sample in proportion to their total numbers in the Netherlands. In addition, patients could only participate through an online questionnaire, which may have led to bias in the composition of our study population.

Participation of health care professionals in the semi-structured interviews was voluntary and recruitment was conducted through a random selection of professionals from the database of the questionnaire study conducted in 2010. It is therefore plausible that this sample also had greater affinity with and involvement in diabetes care than the population of all Dutch care providers, which may limit the generalizability of our findings. Moreover, we selected a heterogeneous sample of health care professionals because of their primary role in diabetes care and in relation to the multidisciplinary approach in Dutch diabetes care. The consequence of including this heterogeneous group was that we were only able to include only two or three professionals per group, and cannot guarantee that saturation has been achieved. Furthermore, we retrieved divergent views on some aspects of care. A broad sample would have been desirable to make an inventory of possible practices and evaluations.

\section{Measurement instruments}

The results of our questionnaire studies are based on self-reported data, which may have led to bias, e.g. through factors related to social desirability. Although self-reports are practical to measure practice performance on a large scale, a review specifically comparing studies of adherence to guidelines using observational data and self-reported data showed that self-reported adherence frequently exceeded the observed adherence (Francke et al., 2008). Therefore, we advocate the use of patient records, objective data and observational data to supplement the self-reports that we used.

Furthermore, we were not able to include an objective clinical outcome measure, such as HbA1c in the studies among patients. It would have been interesting to compare self-reported outcomes in terms of HRQOL with $\mathrm{HbA1c}$ values in patients or assess their association, since previous research recommends using both types of measures and suggests that patients' perspectives can differ from biomedical measures in terms of providing an indication of their disease status (Sundaram et al., 2007). Research into the relationship between patients' objective health status (i.e. glycemic control) and their subjective life quality seems inconsistent (Snoek, 2000). Closely related to this issue, previous studies generally failed to demonstrate an association between generic measures of HRQOL (such as SF-36 or SF-20) and HbA1c, while diabetes-specific 
measures of HRQOL (such as the diabetes quality-of-life (DQOL) measure) have more frequently demonstrated a significant relationship with control of blood glucose (Maddigan et al., 2003; Rubin \& Peyrot, 1999). Combining the use of a disease-specific instrument and a generic instrument may be useful for assessing diabetes HRQOL.

In order to reduce participant burden and increase response rates in the studies among patients, we aimed to limit the number of items of our measure while remaining validity and reliability of the measures. We therefore used subsets of validated questionnaires. Although these subsets of items are not validated as such, the scales show Cronbach's $\alpha$ 's $>0.7$, which indicate a moderate to high internal consistency for these scales in our samples.

The implementation of the CS for diabetes was assessed by asking health care professionals about their familiarity with the CS expressed in whether or not possessing the CS. The question is whether possession is a suitable measure for implementation of the CS, especially since it is questionable whether possessing the CS is a prerequisite for delivering high quality care. Individual health care professionals are subcontracted by care groups, which often appear to develop their own Care Standards and guidelines, but these are typically based on the CS and other evidence-based guidelines. Professionals do however not always seem to be aware of this approach, which results in a low reported familiarity with the CS, whereas the care is still delivered according to the CS in routine practice (Raaijmakers et al., 2010). Therefore, further research into the implementation of the CS for diabetes or other (chronic) diseases, should take these considerations into account when translating the construct of implementation into specific items. Moreover, the results of the studies assessing the implementation of the CS are only based on perceptions of health care professionals rather than objective outcome measures such as referral rates and/or biomedical indicators. Not only these perceptions but also other measures are needed to determine the quality of the implementation of the CS. Nevertheless perceptions of health care professionals are important since they determine their readiness for adopting and implementing an innovation such as the CS (Holt et al., 2010; Moulding, Silagy \& Weller, 1999). In the paragraph on implications for further research this issue will be elaborated upon.

\section{Statistical analyses}

Since we aimed to assess the most predictive and explanatory variables in relation to our study outcomes rather than testing whether a particular model or theory would fit our data, we adopted a regression approach to address our main research questions and we refrained from the application of advanced statistical methods (e.g. Structural Equation Modelling). More complex analyses might have been better suited to make a proper comparison between the data of 2010 and 2013. Matched analyses would have been possible if another study design, i.e. longitudinal, would have been used. In addition, multi-level analyses could have been useful for such a design, since our data has a multi- 
level character because we involved individual professionals nested within care groups. However, in multilevel modeling the statistical power depends on the total sample sizes for each level and sufficient sample size is needed for accurate estimation.

\section{Implications for practice}

Below implications for practice are presented. The implications concern the implementation of Care Standards in the Netherlands, future directions in care and prevention, the counseling style of health care professionals and perceived competence and selfmanagement in patients.

\section{Implications for national action programs}

The implementation of the CS and the NAD took more time and effort than originally anticipated in the NAD. The NAD was planned for a period of four years and aimed the nationwide implementation of the CS. However, four years was not enough for the active dissemination of the CS. Based on the insights of the first monitor study, the NAD developed a series of products to facilitate the implementation, but these products were finished during or at the end of 2012, while the program already finished in June 2013. Developing and publishing a CS does not automatically mean that it will be used in practice. Many policymakers assumed that the implementation would occur on itself, partly due to the function of the CS as a purchasing instrument within the Dutch bundled payment approach for integrated chronic care. However, implementation means more than the adoption and diffusion of innovations (Greenhalgh et al., 2004; Rogers, 2012). Also with regard to the implementation of clinical guidelines, only dissemination of guidelines is not enough; more proactive, multifaceted strategies, for example including educational meetings (Van der Wees et al., 2008), feedback and reminders (Bekkering et al., 2004) are needed to improve guideline adherence. Research into the implementation of innovations teaches us that successful implementation of interventions requires systematic planning during the development phase and that the interventions should made workable and integrated in everyday health care practice (May et al., 2007). In practice, however, the implementation of innovations often seems subordinate to the development of the product itself and more attention for dissemination, adoption and implementation strategies, including adherence, is therefore needed.

Organisations currently involved in developing the Care Standards for other chronic diseases could benefit from existing knowledge and expertise related to the implementation of the CS for diabetes and the facilitating approach used in the NAD pilot regions. Previous research into the dissemination and implementation of interventions has described the tendency of innovators to re-invent the wheel instead of efficiently making use of existing knowledge and insights (Farquhar, Stryer, et al., 2002; Reeves et al., 
2009). To prevent this tendency, a general model has been developed as a framework for the development of Care Standards in the Netherlands (Coördinatieplatform Zorgstandaarden, 2010). An explanation for a possible lack of using expertise in case of the implementation of Care Standards is that organizations and professionals involved in the development of the Care Standards for other diseases were not involved in the implementation process of the CS for diabetes. As a result they did not obtain ownership of the CS. A low degree of ownership is expected to hamper the implementation of innovations (Fleuren et al., 2004). Moreover, successful implementation depends to a large extent on the involvement and investment of both clinical service providers and managers; involvement of relevant staff is necessary at each stage of adoption, implementation and maintenance (Evans-Lacko et al., 2010). Previous research also underlined the importance of taking the specific features of target users into account in interventions aimed at improving guideline adherence (Lugtenberg et al., 2014).

\section{Future directions in care and prevention}

\section{Towards an integrated multidisciplinary approach in chronic care}

Currently in the Netherlands 15 Care Standards (for Asthma among children and adults, cancer, cardiovascular risk management, COPD, dementia, diabetes, obesity, stroke/TIA, traumatic brain injury among adults, and several rare diseases (hereditary breast- and ovarian cancer, hemochromatosis, Hirschsprung's disease, nephrotic syndrome and paraplegia)) have been finished and are implemented (Zorginstituut Nederland). Furthermore, a Care Standard for depression, personality disorders, schizophrenia, traumatic brain injury among children and Li-Fraumeni syndrome are in development. In addition, several Care Standards for mental diseases are planned (Netwerk Kwaliteitsontwikkeling GGz). The currently finished standards are published, but their implementation is not accompanied by an approach such as the NAD and appears to receive less attention than the CS for diabetes. Yet, the CS for obesity and CVRM are available for use within the bundled payment approach.

Despite the multidisciplinary character of the care described in the CS, the CS itself is disease-specific, while multimorbidity is common among (elderly) diabetes patients (Luijks et al., 2012; Marengoni et al., 2011; Sinnige et al., 2013; Uijen \& van de Lisdonk, 2008). Together with the experiences with the implementation of the CS for diabetes this underlines the benefits that could be gained if diabetes care were to move towards an integrated multidisciplinary approach of chronic diseases worldwide. Although other chronic diseases are different in etiology, management and prognosis, the organisation and quality of care needs to meet the same criteria as the care for diabetes and disease management strategies are similar across most chronic diseases. Despite the international differences in health care systems, there is international consensus about the goals of treatment for diabetes and the same type and quality of care needs to be provided to patients with one or more chronic diseases (American Diabetes Association, 
2013). Most approaches are however still disease-specific despite the often multiple health problems among people with chronic conditions (Nolte et al., 2012).

The Care Standards and the bundled payment approach are useful instruments in organising integrated care. In the Netherlands, we have seen multiple collaborations established in the past years aimed at developing an integrated multidisciplinary approach to cope with the rising public health impact of chronic diseases. One of these collaborations concerns an agreement between the partnership Overweight Netherlands, the platform 'Vitale Vaten' ('Vital Veins') and the Netherlands Diabetes Federation. All three organizations focus on providing high quality care for people with (a high risk of) diabetes, cardiovascular diseases and obesity. The aim of this collaboration is to organize the care for these patient groups more effectively and efficiently, in order to increase the quality and patient centeredness of care.

\section{Implementing care and prevention on a local level}

As a result of the promising findings in the NAD pilot regions, a next step in the implementation of the CS for diabetes could be to implement the care on a local level, embedding it into a community approach which also provides opportunities for prevention (of other chronic diseases). Other partners, such as municipalities, municipal health services, health insurers, business, schools and sport clubs also play a role within this approach. This approach is also in line with the spearheads of the policy of the Dutch Ministry of Health, Welfare and Sport. In their most recent note, the ministry described that care and prevention should be provided close to people in their neighborhood which prescribes a community approach (Ministerie van Volksgezondheid Welzijn en Sport, 2011).

It is expected that the growing population of people with multiple long term conditions will demand a different model of care and support; a primarily social model instead of a medical model (Hodgkin \& Taylor, 2013). In the social model patients own resources and the resources of their families and communities should be mobilized to help these patients stay well and functioning. Within this approach it is important to integrate the capacities of caretakers, friends, neighbors, peer supporters, local authorities and the voluntary and community sector. Currently, international trends in obesity and chronic disease prevention move towards an integral approach. The Institute of Medicine in the US recommended the implementation of a comprehensive approach to obesity prevention in the next decade. Within this approach prevention should occur in the environments where people live, work, play, learn and receive health care (Institute of Medicine, 2012). It is expected that the force of action in multiple environmental settings and levels, compounded by the collective ability to accelerate and strengthen each other's impact, can profoundly improve the nation's health. Furthermore, the healthy communities approach in Canada is a local development strategy designed to improve health, welfare, and quality of life of people in their community. It aims to provide the means for populations and communities to ensure greater control over their own well-being 
and health while reducing inequalities in health status (Sasseville et al., 2012). The community approach to chronic disease prevention has a high degree of generalizability, cost-effectiveness, ability to diffuse information successfully through use of community networks, and potential for influencing environmental, regulatory and institutional policies that shape health (World Health Organization, 2014).

In the Netherlands, preventive activities targeting chronic diseases need to become embedded in primary care and close collaboration with the public domain needs to be established within an integrated multidisciplinary approach. In 2012, prevention was added to the CS by means of an addendum. One of the most recent Dutch initiatives for collaboration in prevention of chronic diseases concerns the entry of the Netherlands Diabetes Federation into the National Prevention Program 'Alles is gezondheid'. This program aims to bring professionals and domains together to collaborate and provide care and prevention on a local level, close to the people. The program intends to create a social movement towards evident and permanent health gain (Rijksoverheid, 2014). Primary care in the Netherlands has in recent years started to change its approach from one emphasizing 'care and illness' to one of 'behaviour and health'. The continued implementation of combined lifestyle interventions, such as BeweegKuur (Helmink et al., 2010), can stimulate this change. To ensure the availability of enough exercise facilities for combined lifestyle interventions on a local level, network formation is expected to be a valuable strategy to create closer ties between care and sports. Ideally, these networks should include not only health care providers but also various other disciplines such as municipal government officials, regional public health services, provincial sports councils, welfare workers and local exercise facilities. Close contacts between the various parties are essential to optimize exercise options for each participant. However, a monitor study in 2011 revealed that these ties between care and local exercise facilities were suboptimal (Helmink et al., 2013). The need for network formation and close ties between sectors involved in lifestyle interventions, has initiated two important developments. First, to increase the ties between care and sports and strengthen these networks from the public domain, neighborhood-sport-coaches are introduced as part of the program 'Sport en Bewegen in de buurt' of the Dutch Ministry of Health, Welfare and Sport. This program was established by the Ministry of Health, Welfare and Sport together with several other partners, such as the Association pf Dutch municipalities (VNG), NOC*NSF and the Netherlands Institute for Sport and physical activity (NISB). The main aim of the program is to create a demand-oriented local supply of sport- and exercise facilities and make the healthy choice an easy choice ("Sport en Bewegen in de buurt," 2014). Neighborhood-sport-coaches are specifically assigned to organize sport- and exercise facilities in the neighborhood and connect sport-and exercise facilities with other sectors, such as health care, welfare, youth care, child care and education ("Sport en Bewegen in de buurt," 2014). Second, lifestyle coaches are introduced as new actors in primary care. Combined lifestyle interventions are effective in changing lifestyle related behavior of people with overweight and obesity and related health problems. The beneficial value of 
lifestyle coaches is mainly related to their task of promoting autonomous motivation in people, which is needed for behavioral changes to be maintained by people. Lifestyle coaches play a pivotal role in the treatment of lifestyle related health problems in primary care, but also for the treatment of children with overweight in secondary care. The lifestyle coach helps people change their lifestyle through individual counseling and tailored advice. The coach counsels people with a health risk, advices about possible treatment programs, maintains contact and motivates people. Individually tailored attention is assumed to help people maintain their lifestyle changes. In addition, the lifestyle coach initiates collaboration between primary care, secondary care and the local exercise facilities and is in charge of directing network formation and maintenance.

The results of the questionnaire studies among health care professionals described in this dissertation showed that the lack of effective lifestyle interventions (or the access to them) was one of the most important perceived barriers (Raaijmakers et al., 2010; Raaijmakers, Martens, Hesselink, et al., 2013). Programs meeting requirements for costeffective interventions, in terms of being at least well underpinned, have been developed in the Netherlands and have been implemented in several pilot projects in primary care, examples being 'BeweegKuur' (Helmink et al., 2010), 'COACH methode' (Leemrijse et al., 2009) and 'Exercise on prescription' (Hosper et al., 2008). The familiarity with such programs, as well as access to them, should obviously be improved. A registration and assessment system for health education and health promotion interventions has been developed in the Netherlands in an attempt to promote quality assurance and control (Brug et al., 2010). Whether this system will help ensure that the most effective and efficient interventions are implemented and disseminated can as yet not be guaranteed (Algemene Rekenkamer, 2010), but at least a comprehensive national list is being put together.

\section{Attention for personalized care}

Many of the ingredients for an integral multidisciplinary approach of care and prevention for chronic diseases are already in place (Hodgkin \& Taylor, 2013).

E-health can be a useful tool to provide personalized care for patients with a chronic illness. The rise of internet and e-health has been recognized to provide opportunities for patient empowerment (Samoocha et al., 2010). The Dutch Ministry of Health, Welfare and Sport also acknowledges the opportunities of e-health, but also identified adjustment to e-health by patients and professionals as a precondition (Krijgsman et al., 2013). E-health can also facilitate shared decision making between the patient and health care professional(s) since it can play a role in preparing the patient and heath care professionals to the interaction with each other. In order to be successful, the patient needs to be open to participate in the decision process. Interventions aimed at changing individuals' health behaviors and increasing patients' self-management skills showed that e-health applications can be useful (Kuijpers et al., 2013; Nijland et al., 2011; Wantland et al., 2004). However, a possible pitfall of using e-health tools, is that 
the time used for consultation with the health care professionals will mainly be oriented towards medical issues, for example as a result of focusing on HbA1c values instead of discussing self-management and motivational issues, which are already covered by the e-health application. This pitfall is also an issue within the bundled payment approach. The contracts are focused on quality indicators and parameters and these are usually medically oriented. As a result, professionals are not directly rewarded for applying a certain counseling style to empower patients in applying self-management. However, the results of this dissertation underline the importance of self-management in patients, also in relation to their $\mathrm{HRQOL}$, an equally as important indicator of patients' disease status as biomedical indicators (Sundaram et al., 2007). Therefore, the quality of ehealth tools should be further improved to tackle this pitfall, for example by incorporating feedback systems that help professionals to discuss non-medical issues during consultation and empower patients in self-managing their disease, and tools that empower patients to set the agenda for consultations with their physician also including selfmanagement or psychosocial issues.

\section{Counseling style of health care professionals}

\section{Autonomy supportive versus directive}

This dissertation shows that perceived autonomy support is important in relation to diabetes patients' self-management, general diabetes control and HRQOL. Professionals can be trained to be autonomy-supportive using person-centered approaches, such as motivational interviewing (MI). SDT and MI have the same basic assumption that people have a strong potential (inner resources) to realize change (Patrick \& Williams, 2012; Vansteenkiste \& Sheldon, 2006). From a SDT perspective, it seems that MI techniques facilitate the process of internalization of extrinsic motivation or greater integrated motivation for change, rather than enhancing peoples' intrinsic motivation as described by Miller and Rollnick (Miller \& Rollnick, 2002). Counseling for both MI and SDT (autonomy support), can be characterized as eliciting or drawing out motivation from people by supporting their inner resources (Vansteenkiste \& Sheldon, 2006). Autonomy support is central to the practice of MI (Deci \& Ryan, 2012; Markland et al., 2005), and previous research showed that patients were more likely to perceive a high degree of autonomy support when their physicians used reflective statements, one of the key elements of $\mathrm{MI}$ (Pollak et al., 2011; Resnicow \& McMaster, 2012).

In practice, the importance of $\mathrm{Ml}$ is recognized and professionals attend training in $\mathrm{MI}$, which often lasts one day or afternoon; sometimes a 3-day course is followed. Previous research revealed that 2 to 3 day training may not be sufficient for counselors to adhere to MI (Groeneveld et al., 2011). According to Miller et al. such training can increase professionals' understanding of $\mathrm{Ml}$ and make them acquire the basic skills, but to become an expert, personal feedback or a more advanced training is necessary (Miller et al., 2004). Therefore, the implementation of MI in practice is expected to need more 
time, effort, practice sessions and feedback in order to become part of professionals' routine, although minimal improvement of nurses' motivational interviewing skills in routine diabetes care have been found in a cluster randomized trial one year after following 16 hours of training (Jansink et al., 2013). Moreover, literature on the effects of brief $\mathrm{Ml}$ interventions on a variety of outcomes is inconsistent. Some studies in diabetes care have shown that $\mathrm{Ml}$ is effective in lifestyle change (Britt et al., 2004; Brug et al., 2007; Martins \& McNeil, 2009) and that it has beneficial effects on several clinical outcome measures (Rubak et al., 2005). However, other studies showed no effect of $\mathrm{MI}$ on HbA1c in general practices (Brug et al., 2007; Rubak et al., 2005), and no effect on the lifestyle, clinical parameters, quality of life and self-efficacy (Heinrich et al., 2010; Koelewijn-van Loon et al., 2009).

With regard to studies assessing the implementation of $\mathrm{Ml}$ among professionals, results of a cluster randomized trial showed that the maintenance of the Ml skills of trained general practice nurses was minimal one year after a training program consisting (Jansink et al., 2013). Furthermore, a study among nurse practitioners in primary care aimed to implement a lifestyle program showed that motivational interviewing was the most challenging aspect of the protocol that had to be implemented by these nurses (Whittemore et al., 2009).

Another autonomy supportive technique is shared decision making. Shared decision making is defined as a process by which a health care choice is made jointly by the health care professional and the patient and is said to be the core of patient-centered care (Makoul \& Clayman, 2006; Towle \& Godolphin, 1999). Shared decision making can facilitate diabetes patients in deciding to use self-management or develop these skills to self-regulate their diabetes. Therefore, this technique can be a safe starting point to increase patients' autonomy. To increase the adoption of shared-decision making, health care professional training may be important (Legare et al., 2010; Stiggelbout et al., 2012), as may be the implementation of patient mediated interventions such as decision aids (Legare et al., 2008).

In contrast to the focus on autonomy supportive counseling, the results of this dissertation also imply that some patients may prefer a directive approach, in which the doctor gives more advice and tells the patients what to do rather than a patientcentered autonomy supportive approach in which the doctor gives little information and asks the patient what he/she wants (Deci \& Ryan, 2012; Vansteenkiste et al., 2012). Such an approach is underlined by research that indicated that engaging in self-management behaviors only has a minor effect on perceived health, quality of life and diabetes control (Maddigan et al., 2005; Watkins et al., 2000). Moreover, the case study of Schipper et al. (2011) using stories of a professional caregiver and a client with acquired brain injury showed that being dependent and asking for help may give patients a greater sense of being autonomous than being independent and doing things without help (Schipper et al., 2011). This study also implies that daily support of patients should not be focused solely on autonomy, but also on recognition of dependency in patients. Pro- 
fessionals need to understand and respect each other's values in order to increase patient autonomy and they should readjust their counseling style to patients' needs and wishes (Schipper et al., 2011).

Altogether, the possibility for practitioners to tailor their counseling style to the patients' individual needs for directives and autonomy support could be useful in providing the care that fits best with the needs of the patient and is most effective in terms of invested time and effort by the health care professional (Deci \& Ryan, 2012). A balance between autonomy and controlled motivation can be considered, since a low level of controlled motivation does not seem to limit patients' abilities to self-manage their behavior (Ryan \& Deci, 2000). Furthermore, research into shared decision making suggested that a similar approach to all patients is not likely to meet patients' wishes, since preferences for participation vary among patients (Brom et al., 2014). Moreover, autonomy supportive counseling styles are expected to take more time and training from health care professionals as described above. Previous research shows that time constraints remain the most often perceived barrier for implementing shared decisionmaking in clinical practice across many different contexts, despite the lack of strong evidence that shared decision-making takes more time than usual care (Legare et al., 2008). Implementing shared decision making based on patient characteristics was perceived as a barrier as well; suggesting that health professionals might be screening which patients will prefer or benefit from shared decision-making beforehand. However, this raises some concerns since professionals may misjudge patients' desire for active involvement in decision-making (Gravel et al., 2006). Screening patients should therefore be done by using validated tools and not depend solely on health professionals' evaluation of the patient desires.

\section{The role of patients' level of mastery}

On the basis of our results, we assume that patients with a greater sense of mastery might recognize an autonomy-supportive counseling style more clearly or may even induce such a counseling style in health care professionals. On the other hand, patients with a lower sense of mastery are assumed to have a preference for a more directive counseling style. This is underlined by the finding that patients with a lower sense of mastery perceived less autonomy support from their primary caregiver (Chapter 5).

According to SDT, mastery influences people's motivational regulation. A higher sense of mastery is assumed to increase patients' autonomous motivation. A study among renal patients, who were engaged to list priorities for social scientific research in order to complement the professionals' research agenda, showed that having some influence on their own life and treatment was listed as a third top priority by these patients. Patients indicated that they want to maintain control over their lives. This does not mean that they want to do or decide everything alone and without the help of others (positive autonomy). Rather, it means being the master of your own life, having control and directing your life as much as possible. This can also mean that choices are 
made with help of others and being dependent (negative autonomy). In short, research should be focused on how patients can obtain mastery over their life, with or without help of others (Schipper \& Abma, 2011).

Mastery can be regarded as a dispositional personality trait (Deci \& Ryan, 1985) and therefore may not be easy to modify. However, its influence on patients' motivational regulation and causality orientations can be a target for interventions that focus on aspects like changes in the counseling style of health care professionals. One option would be to tailor the counseling style to the patient's mastery level. Health care professionals could readjust the personal goals of patients with a low sense of mastery. A recent review about nutrition and physical activity guidance offered in general practices found a lack of assessment of patients' barriers and supporting factors in counseling (Van Dillen et al., 2013). A previous intervention study also concluded that health professionals should take mastery perceptions of diabetes patients into account during regular consultations, with a view to improving treatment acceptance and adherence (Fall et al., 2013).

On the other hand, mastery can be viewed as a modifiable state (Rose et al., 2005; Schieman \& Turner, 1998). Rose et al. explored the relationship between causality orientations, behavioral regulation for exercise and stage of change for exercise and showed that levels of the autonomy orientation can change and were related to time spent participating in exercise (Rose et al., 2005). This result indicates that individuals with low levels of autonomy can be placed in an environment that will promote autonomy and this will motivate individuals through a behavior change process. From this point of view, mastery can be changed during the lives of patients and lifestyle interventions aimed at behavioral change and improving patients' skills and attribution style could be used to improve mastery. Referral to a psychologist for cognitive behavioral therapy (CBT) or integrating concepts of CBT in tailored self-management interventions could increase patients' sense of mastery over their life and control over their disease. Previous research showed that personal perceptions are important motivators of self-care behaviors (Peyrot \& Rubin, 2007) and comprise a core principle of CBT (Beck, 1993). Changing patients' perceptions has proven to be effective in changing diabetes-related outcomes (Keogh et al., 2011). Furthermore, elements of reattribution training could be integrated in self-management interventions as well, or in the counseling style used for patients with a low sense of mastery.

\section{Perceived competence and self-management in patients}

Chapter 4 of this dissertation underlines the importance of perceived competence in relation to self-care activities and general diabetes control of patients with type 1 and type 2 diabetes. Improving patients' perceived competence seems beneficial for their self-care activities, especially with regard to following a healthy diet. Previous studies found that increasing perceived competence leads to greater motivation for diabetes self-care and better glucose control (Williams et al., 1998; Williams et al., 2004). Per- 
ceived competence is facilitated by autonomous self-regulation, which arises out of need-supportive contexts.

Chapter 4 further reveals that perceived competence mediates the influence of autonomy support, especially in type 1 diabetics, while in type 2 diabetics autonomy support remains to have a statistically significant explanatory contribution in the final model. The results indicate that health care professionals do not completely manage to influence perceived competence in type 2 patients by providing them autonomy support in contrast to type 1 patients where they are successful. This is in line with our expectations since diabetes type 1 is generally diagnosed at a relatively young age, so patients learn to live with their diabetes earlier in life, which is expected to be easier. Overall, type 2 patients are diagnosed at a later age, which probably makes it more difficult to adjust their lives to their chronic disease.

Our findings contribute to the already available body of evidence of the importance of perceived competence in relation to diabetes self-care and better glucose control (Williams, Freedman, et al., 1998; Williams et al., 2004). Perceived competence has been shown to be involved in both the adoption of and long-term adherence to selfmanagement behaviors (Williams \& Bond, 2002). SDT assumes that perceived competence alone however is not sufficient to motivate behavior and should be accompanied by autonomy. In contrast, Social Cognitive theory places nearly exclusive emphasis on self-efficacy, a concept that is closely related to perceived competence (Patrick \& Williams, 2012). Altogether, perceived competence can be viewed as precondition for change. Strategies for enhancing perceived competence and self-efficacy include identifying and setting realistic goals, problem-solving to reduce barriers to goal achievement, coping with unchangeable circumstances, and identifying and eliciting appropriate social support (e.g., Anderson et al., 1995; Strecher et al., 1995). Since the Individual Care Plan and e-health applications can support patients and providers to set collaborative goals and discuss and follow-up these goals, these tools can be useful to enhance patient's perceived competence (Williams et al., 2007). Shared decision making can also contribute to increasing patient's perceived competence, since it puts clear emphasis on their own choice and autonomy (Towle \& Godolphin, 1999).

This dissertation showed that both autonomy support and perceived competence are important in relation to patients' self-management and HRQOL. Therefore, intervention strategies aimed at improving both autonomy and competence could provide diabetes patients with an environment that allows informed choices about self-care behaviors (such as following a healthy diet) and diabetes, enhances the ability to identify and set realistic goals, and enhances problem-solving capabilities and coping potential (Dyck et al., 2006). 


\section{Implications for theory}

This dissertation also provides some insights which have implications for theory formation and use in diabetes care. The results of the studies conducted among patients confirm the usefulness of SDT concepts in relation to self-management and motivation in Dutch diabetes patients. SDT has been proven to be highly useful in explaining motivation of people with a chronic disease in being physically active (Teixeira et al., 2012) and complying with their treatment (Williams et al., 2004; Williams et al., 2009). Furthermore, the study described in chapter 4 showed that mastery is an important associate of HRQOL in diabetes patients. The question is how this concept can be integrated in existing theories predicting people's motivation. The results on mastery in diabetes patients can be considered from a broader Self Determination Theory (SDT) perspective, since the concept of mastery is closely related to the impersonal orientation of behavioral regulation (Deci \& Ryan, 1985). SDT proposes that people have dispositional causality orientations that refer to (a) the way people orient to the environment as regards information related to the initiation and regulation of behavior, and (b) the extent to which they are self-determined in general, across situations and domains (Deci \& Ryan, 2008). Individuals can be oriented by each of three general sources of behavioral regulation: autonomy, control and impersonal orientation. People with high scores for the impersonal orientation see themselves as incompetent and unable to master situations (Deci \& Ryan, 1985). A previous study by Williams et al. (1996) showed that morbidly obese patients' autonomous motivation for weight loss and engaging in a diet program was predicted by their autonomy orientation (Williams et al., 1996). In addition, impersonal orientation proved to be related to an increase in controlled motivation and a decrease in subjective well-being among older adults in a 16-week randomized exercise trial (Solberg et al., 2013). Rose and colleagues supported the importance of the autonomy orientation for motivating adult individuals to adopt and maintain exercise (Rose et al., 2005). Furthermore, SDT assumes that autonomy support is important in relation to people's motivation. The question is however, whether this assumption fits the needs of all types of patients. Our results suggest that some patients may prefer a directive approach, (traditional model) rather than provided with autonomy support.

In the study described in chapter 3, we used the CCM as the underlying theoretical framework and we acknowledged the usefulness of the Expanded CCM which emphasizes the importance of health promotion and integrating prevention within primary care (Barr et al., 2003). The increasing incidence of chronic diseases and its burden on individuals and health care systems underlines the importance of embedding prevention in health care. A major difference in the expanded model is the emphasis on porous borders between the health care system and the community, which also fits the increased attention of initiatives in which care and prevention should be provided using a community approach (Ministerie van Volksgezondheid Welzijn en Sport, 2011). This dissertation indicates however that expansion of the Expanded CCM (Barr et al., 2003) could be use- 
ful. The porous borders in the expanded model already implicate that health care and the public domain/community are closely related. A next step could be to integrate both domains by concretely incorporating the element of intersectoral collaboration. The 'Behavior Change Ball', a tool to study the development and implementation of integrated public health policies within local governments, captures this element (Hendriks et al., 2013). Intersectoral collaboration can facilitate crossing the border from the care domain to the public domain. As previously described, primary care in the Netherlands has in recent years started to change its approach from one that is emphasizing 'care and illness' to one of 'behaviour and health'. This means that the collaboration between the care domain and public health domain should be established in order to facilitate patients in accessing lifestyle interventions and other preventive activities, often provided within the public domain. However, ties between both domains have been shown to be suboptimal (Helmink et al., 2013).

\section{Implications for further research}

In addition to recommendations for practice and theory, some implications for further research, related to study design, measurement instruments and theoretical concepts, are outlined below.

\section{Study design}

This dissertation underlines the usefulness of action research in relation to the implementation of innovations in health care. The results of the studies conducted to monitor the nationwide implementation of the CS were fed back to the implementation institute (NDF/NAD) and used to adapt intervention contents and implementation strategies. In this respect, the series of studies we conducted can be viewed as action research (Koshey et al., 2011), which is characterized by involving professionals in order to help them improve practice. It includes action, evaluation, and critical reflection and - based on the evidence gathered through research - new objectives are formulated and changes in practice are then implemented (Koshey et al., 2011). In our studies, health care professionals and representatives of the NDF and the NAD implementation team were involved from the start. The development of the measurement instruments occurred in collaboration with members of the NDF and pretests were conducted among health care professionals from all disciplines involved. In addition, a communication plan for the recruitment of professionals and patients was developed in collaboration with the NDF. Furthermore, preliminary results of the studies were presented at meetings with the NAD implementation team and board members and to employees of the NDF in order to support their objectives and adapt their actions in practice. In return, feedback from these representatives was used to further interpret the results and inform questions 
asked in qualitative studies. A limitation of this type of research is the restricted inclusion of theoretical constructs to explain underlying mechanisms of the results.

\section{Measurement instruments}

Based on research assessing adherence to clinical guidelines, knowledge and acceptation of and adherence to elements of the CS can be measured using different indicators besides self-reported perceptions. A distinction can be made between structure, process and outcome indicators and qualitative as well as quantitative methods can be used (Kennisbank richtlijnontwikkeling). In addition to the self-report instruments used in this dissertation, registration forms or logbooks can be useful to obtain more insight in professionals' self-reported adherence to the CS. Usually these instruments are used over a longer period of time and completed prospectively, which leads to a more accurate reflection of the fluctuations in implementation compared to questionnaires (Fleuren et al., 2014).

Further research should also use more objective information from electronic patient files or general practices, the bundled payment information system or specific registration systems such as the National Medical Registration to assess the quality of care provided and adherence to the CS (Kennisbank richtlijnontwikkeling). A next step could also be to assess the implementation of the CS and its quality through observational studies in practices and hospitals. Direct observation or audio- or videotaping of consultations can be seen as the gold standard but is time consuming and expensive to implement, even in research settings. Recent studies in the Netherlands used videotaped real-life practice nurse-patient consultations to examine the content of Dutch primary care practice nurses' (PNs') lifestyle advices for overweight or obese patients (Van Dillen et al., 2014) and for hypertension patients (Milder et al., 2008), and the application of Motivational Interviewing by PNs in diabetes consultations (Jansink et al., 2013) and in targeting lifestyle behaviors (Noordman et al., 2013a; Noordman et al., 2013b). Another method to assess adherence to the CS and performance of professionals is the use of vignettes. Vignettes consist of 'text, images or other forms of stimuli to which research participants are asked to respond' and seem to offer a promising alternative for the assessment of professionals' performance (Hughes \& Huby 2002). Vignettes (in the form of written case simulations) have been used in a variety of settings (Hughes \& Huby 2002), such as physiotherapy (Rutten et al., 2009), mental health (Falzer \& Garman, 2010; Tiemeier et al.2002) and oncology (Holland-Barkis et al., 2006) and also in diabetes care (Williamson et al., 2013). A few studies have shown that clinical vignettes are suitable for measuring clinical performance (Rutten et al., 2006; Peabody et al., 2004; Peabody et al., 2000) and conceptual use of guidelines (Fleuren et al., 2014). The use of vignettes can also be combined with measures of perceptions to be able to compare actual adherence with self-reported adherence, since previous research has shown misperceptions about adherence to evidence-based treatment recommendations among professionals (Rutten et 
al., 2009). The use of video vignettes may also be promising in future studies assessing professionals' performance of elements of the CS. Currently, video vignettes are mainly used in research assessing patient-provider communication, and this method is still in development (Hillen et al., 2013).

In this dissertation we assessed the perceptions of health care professionals towards the CS, while the importance of involving the perspective of care groups was also acknowledged (Chapter 7). Involving these care groups on a larger scale in future studies assessing the implementation of and perceptions regarding Care Standards and guidelines, similar to studies on the Bundled Payment system in the Netherlands (Lemmens, 2012) would therefore be useful. Questionnaire studies or qualitative studies using semistructured or in-depth interviews can be used to obtain insight in processes from the perspective of care groups.

\section{Theoretical concepts}

Implementation of the CS

In relation to the limitation concerning the assessment of the implementation of the CS, as previously described in this chapter, further research into the implementation of other (future) Care Standards should think about redefining and/or extending the assessment of implementation in terms of possession of the CS. Previous research assessing the degree of implementation/use of clinical guidelines has used different measures on three main dimensions of use (quantitative, qualitative and conceptual use) (Fleuren et al., 2014). Most commonly used measures are the frequency of use (number of times used; sometimes expressed in level of use), the frequency of conducting a core element during a certain period, exposure to the guideline (reach), the completeness of use (applied proportion of recommended core elements), the duration of use, the time needed to conduct a core element and the way professionals conduct core recommendations (Fleuren et al., 2014). Furthermore, fidelity is an important indicator of implementation (Carroll et al., 2007).

Previous research regarding professionals' adherence to clinical guidelines can serve as input for the assessment of the implementation of and adherence to Care Standards. Professionals may perceive the CS as an innovation implying the relevance of the Diffusion of Innovations Theory of Rogers (2003). Rogers distinguishes five stages in the innovation decision process. The first two stages (knowledge and persuasion) refer to the dissemination process. The other three are behavioral stages and part of the adoption process. Potential adopters have to decide whether to adopt or reject the innovation. The subsequent implementation stage focuses on actual use; suitable measures for use of Care Standards are discussed in the previous paragraph. Perceived barriers are also important in this stage and covered in this dissertation. Finally the innovation becomes part of the working routine in the confirmation stage (Rogers, 2003). Rogers' theory has been used in previous studies assessing (determinants of) adherence to clinical guide- 
lines (e.g. Davies et al., 2010; Harting et al., 2009; Hader et al., 2007). Moreover, the relevance of combining theoretical models that jointly address the entire disseminationto-implementation spectrum has been underlined (Tabak et al. (2012). Therefore, the framework of determinants of innovation processes described by Fleuren et al. (2004) can be useful in future research assessing the implementation of Care Standards. According to this framework, the processes of adoption, implementation and continuation are influenced by characteristics of the innovation itself, characteristics of adopting organization and characteristics of the sociopolitical context. The relations between these characteristics and the innovation decision process are mediated by characteristics of the user, while the relation between the characteristics of the user and the innovation decision process is moderated by the characteristics of the dissemination strategy. The framework has been used in a number of empirical studies in Dutch health care (e.g. Huijg et al., 2014; Verweij et al., 2012; De Veer et al., 2011).

Another concept that deserves more attention in relation to adherence to guidelines or Care Standards is that of awareness of personal performance, since it may interfere with the relationship between determinants of adherence and actual guideline adherence (Rutten et al., 2009).

It would also be interesting to include constructs of behavioral change models in further research on the implementation of Care Standards, since there may be a lack of theoretical understanding of the processes involved in changing the behavior of health care professionals (Michie et al., 2005). Moreover, different domains of behavior change (i.e. knowledge, skills, behavioral regulation) have been identified in relation to the implementation of clinical guidelines and evidence based practices (Michie et al., 2005).

Additionally, an approach in which implementation strategies are based on identified barriers to change in health care could be useful. Most theories on implementation of evidence in health care underline the importance of obtaining good insight and understanding of these barriers to develop effective interventions (Baker et al., 2010; Bosch et al., 2007; Grol \& Grimshaw, 2003). Moreover, previous studies that analyzed several of these barriers, have shown that they operate at different levels in the health care system (i.e. the level of the patient, the individual professional, the health care team, the health care organization or the wider environment) (Baker et al., 2010; Cabana et al., 1999; Grol et al., 2003; Grol \& Grimshaw, 2003; ). It would therefore be useful to expand the assessment of the implementation of Care Standards among health care professionals and patient with assessment on more levels, i.e. on the level of care groups, health centers, hospitals and multidisciplinary teams in secondary care.

Finally, to improve understanding of the implementation process of the CS for diabetes and other Care Standards the Consolidated Framework for Implementation Research (CFIR) can be useful in further research (Damschroder et al., 2009). The CFIR comprises five major domains: the intervention, inner setting, outer setting, the individuals involved, and the process by which implementation is accomplished. The first major domain of the CFIR is related to characteristics of the intervention being implemented 
into a particular organization. The next two domains in the CFIR are the inner and outer setting. Changes in the outer setting (generally including economic, political and social context) can influence implementation, often mediated through changes in the inner setting, which includes characteristics of structural, political, and cultural contexts through which the implementation process will proceed (Institute of Medicine, 2001; Pettigrew, Woodman \& Cameron, 2001). The fourth major domain of the CFIR includes the individuals involved with the intervention and/or implementation process. Individuals make choices and are carriers of cultural, organizational, professional, and individual mindsets, norms, interests, and affiliations. The fifth domain is the implementation process. Successful implementation usually requires an active change process aimed to achieve individual and organizational level use of the intervention as designed. The CFIR provides a pragmatic framework that can be used to expand the knowledge on implementation across different settings (Damschroder et al., 2009).

\section{Perspective of patients}

Further research among patients should be aimed at examining the underlying mechanism of the associations between mastery and self-management and HRQOL in diabetes patients. Furthermore research should identify risk groups based on personal characteristics, in order to recognize patients with low mastery levels and to tailor the counseling style to these patients. Moreover, previous research showed that mastery can be regarded as a dispositional personality trait (Deci \& Ryan, 1985) and therefore it may not be easy to modify. On the other hand, mastery can also be viewed as a modifiable state (Schieman \& Turner, 1998). Research should examine which of both assumptions is the best way to approach mastery or how both ways can be optimally and mutually used in order to increase patients' mastery level or tailor the counseling style of professionals to patients' mastery.

The results of the study described in chapter 4 showed that perceived competence appears to mediate the influence of autonomy support, especially in type 1 diabetics, while in type 2 diabetics autonomy support remains to have a statistically significant explanatory contribution in the final model. Further research into the relation between perceived competence and perceived autonomy support with self-management in diabetes patients using a longitudinal design with path analysis is therefore recommended. Specific aims should be to assess this possible mediating mechanism of perceived competence in the association between autonomy support and self-management. Moreover, studies using a longitudinal design can provide further insight in the causality of the associations. Ultimately this is needed for the development of interventions to improve patients' self-management skills, since these are expected to be of increased importance in keeping chronic diseases manageable and care affordable in the future.

In addition, research focusing on differences in the underlying mechanism and determinants of self-management and HRQOL between type 1 and type 2 diabetics is needed. Chapter 4, which showed differences in the association between autonomy 
support and self-management and general diabetes control in type 1 and type 2 diabetics, provides concrete leads to further assess these patient groups separately. Patients with type 1 and type 2 diabetes may differ in their needs regarding self-management and treatment since these illnesses are different in etiology, management and prognosis. To our knowledge, however, no previous studies assessed differences between both patient groups in this respect.

\section{General conclusion}

The current dissertation aimed to provide a closer look at diabetes care and the introduction of the Care Standard from the perspectives of health care professionals and diabetes patients. From the studies conducted among health care professionals it can be concluded that CS seems to serve as a supporting tool for professionals to organize diabetes care and increases awareness of what high quality care means. Moreover, the NAD has resulted in the CS to become embedded in the Dutch health care system and the introduction of the CS, supported by the NAD, and the bundled payment approach as a parallel development, has provided momentum for the realization of various facilitating processes relating to the wider implementation of standards to improve the care for people with other chronic diseases in the Netherlands. Further research is needed to determine whether the CS is truly necessary for delivery of high quality care and whether the CS is a necessary addition to the guidelines of the individual professionals groups. The studies among patients underline the importance of mastery in relation to patients' perceived autonomy support from their primary caregiver, self-management skills and HRQOL. Furthermore, autonomy support and motivational interviewing, together with strategies for enhancing perceived competence, are important elements to include in interventions aimed at improving adherence to self-care behaviors and enhancing quality of life.

Altogether, diabetes care has undergone several changes aimed to improve the quality of care. The experiences with the implementation of the CS for diabetes and the NAD underline the beneficial value of the ongoing process in which healthcare moves towards an integrated multidisciplinary approach of chronic diseases worldwide which incorporates personalized care that fits the patients' needs and characteristics. 


\section{Valorization addendum}

In addition to the scientific value of this thesis described in Chapters 2-8, the results of the research presented also have societal and economic value. This value will be described in this valorization addendum and specified in terms of the relevance of the study results, target groups to whom the results are of interest, the translation of the study findings in activities and products, the innovative character of these activities and products and the planning of the valorization of the results.

\section{Relevance}

Diabetes mellitus is a rapidly growing health problem, which affects approximately 382 million people worldwide (International Diabetes Federation, 2013). In the Netherlands, 834,100 people were diagnosed with diabetes on January 1st, 2011 and this number increased by 87,000 patients in that same year (Baan et al., 2014). Diabetes is a multifactorial health problem that requires a multidisciplinary approach to prevention and treatment. As in most industrialized countries, Dutch health care faces the challenge of guaranteeing continuity and quality of care for the growing number of people with diabetes. For this reason multiple changes in diabetes care have been introduced in recent years; one of these concerns the introduction and implementation of the Netherlands Diabetes Federation (NDF) Care Standard (CS) for diabetes. The NDF CS for type 2 diabetes mellitus describes the norm for generic multidisciplinary diabetes care and focuses on the content, organization and quality of diabetes care (Nederlandse Diabetes Federatie, 2013b). The overall aim of implementing the CS is to improve the quality and organization of care for diabetes patients in order to achieve better outcomes in patients. The studies presented in this dissertation are unique in monitoring the nationwide implementation process of this CS and the National Diabetes Action Program (NAD) that facilitated this process. Our results show that the CS seems to serve as a supporting tool for professionals to organize diabetes care and it makes professionals and other relevant stakeholders aware of what high quality care means. This dissertation provided concrete insights in the implementation of the CS and perceived barriers and points for improve- 
ment, which may eventually lead to better outcomes on the level of patients. Moreover, the studies among patients have provided concrete leads to optimize the counseling style of health care professionals in order to improve patients' self-management and health related quality of life. Factors associated with these improvements have also been examined separately for type 2 and type 2 patients. This is especially relevant, since the counseling style and treatment for both patient groups may be different. The improvements in care and counseling are expected to lead to more satisfied patients, better health outcomes and eventually a decrease in health care costs on the long term.

\section{Target groups}

The results presented in this dissertation are of interest to several target groups and sectors in society. First of all, the results of the studies are of course directly relevant for all health care professionals involved in diabetes care. The results of the studies among patients provide concrete leads for health care professionals to optimize and tailor their counseling style to the individual needs and characteristics of patients.

The information in this dissertation can also be useful for professionals involved in the care for other chronic diseases and more specifically for professionals and organizations involved in the development and implementation of Care Standards for other (chronic) diseases in the Netherlands.

Third, diabetes patients are a highly relevant target group. The overall aim of the CS is to improve the quality of care for people with diabetes. The studies assessing the implementation of the CS can therefore contribute to improving the care for patients. Moreover, the studies among patients provided useful insights in factors associated with self-management and health-related quality of life in type 1 and type 2 diabetics. Furthermore, we examined patients' familiarity with and attitude towards the Zorgwijzer, a version of the CS especially for patients with diabetes, which explains what they can expect from their health care providers. A Zorgwijzer can empower patients and can support their self-management skills (Ploeg, 2011).

Fourth, the results of the studies might be of interest to care groups. Although not originally developed for this purpose, the CS is used as a purchasing instrument in the Dutch bundled payment approach for integrated chronic care (Struijs \& Baan, 2011). This approach has laid the foundation for delivering and funding diabetes care in accordance with the CS and has also partly led to the development of care groups. Within this approach, insurers purchase the services and care described in the CS from a general contractor called a care group, which ends up in a bundled payment contract. These care groups are relatively new to the health care system and have been established to improve the quality of chronic care (Inspectie van de Gezondheidszorg, 2012). A care group consists of several health care providers that form a legal unit. Based on the bundled payment contract, the care group takes clinical and financial responsibility for all assigned patients in diabetes care and in turn subcontracts to individual care providers 
(e.g. general practitioner, dietician, internist etc.) or delivers parts of the services in relation to the various components of diabetes care itself (Struijs \& Baan, 2011). The CS is thus a prerequisite for applying the bundled payment approach in health care and the bundled payment contracts prescribe what care will be financed. The purchase function of the CS within this approach is primarily used on the level of care groups. Moreover, care groups often appear to develop their own Care Standards and guidelines, but these are typically based on the CS and other evidence-based guidelines. The care groups are also responsible for implementing these standards. The results regarding perceptions of health care professionals and perceived barriers in relation to the CS can provide concrete leads for care groups to improve the implementation process.

Fifth, the results are also interesting to health insurers. Within the bundled payment approach in health care the CS serves as a financial incentive in the negotiations with health insurers.

Sixth, organizations currently involved in developing the Care Standards for other chronic diseases could benefit from existing knowledge and expertise related to the implementation of the CS for diabetes. The currently finished standards for Asthma among children and adults, cancer, cardiovascular risk management, COPD, dementia, diabetes, obesity, stroke/TIA, traumatic brain injury among adults, and several rare diseases (hereditary breast- and ovarian cancer, hemochromatosis, Hirschsprung's disease, nephrotic syndrome and paraplegia)) have been published, but their implementation is not accompanied by an approach such as the NAD and appears to receive less attention than the CS for diabetes. The National Health Care Institute, responsible for stimulating and coordinating the development of Care Standards, can also use the results of this dissertation in further guiding the development and implementation of other Care Standards.

Finally, the results might also be of interest from a political point of view. The dissemination and implementation of the CS for diabetes has functioned as a pilot and accompanying the implementation of the CS with a governmentally funded Action Plan may has been an example of a best practice. The results provide learned lessons and points for improvement for the development, dissemination and implementation of future Care Standards in the Netherlands.

\section{Activities and products}

The NAD has resulted in the first Care Standard to become embedded in the Dutch health care system. The results of the studies to monitor the implementation of the NAD and CS were fed back to the implementation institute in order to support their objectives and actions in practice. For this purpose, two reports were written and four presentations and three feedback sessions were given. The NDF used the results to adapt implementation strategies and to design over 50 products used to facilitate the implementation of the CS. These products were related to the NAD themes and based on existing 
barriers in practice, amongst others identified in the studies included in this dissertation. The NDF selected five pilot regions in the Netherlands to initially implement several of these products with the objective of improving them and then implementing them nationally in the future.

\section{Innovation}

The studies presented in this dissertation are unique in monitoring the nationwide implementation process of the CS for diabetes and were part of an explorative trajectory. The CS for diabetes was the first CS to be implemented in the Netherlands and the results of the studies included in this dissertation can be used to aid the adoption and implementation of Care Standards for other chronic diseases in the Netherlands and to inform similar approaches in other countries. Currently in the Netherlands 15 Care Standards (for Asthma among children and adults, cancer, cardiovascular risk management, COPD, dementia, diabetes, obesity, stroke/TIA, traumatic brain injury among adults, and several rare diseases (hereditary breast- and ovarian cancer, hemochromatosis, Hirschsprung's disease, nephrotic syndrome and paraplegia)) have been finished and are implemented (Zorginstituut Nederland). Furthermore, a Care Standard for depression, personality disorders, schizophrenia, traumatic brain injury among children and LiFraumeni syndrome are in development. In addition, several Care Standards for mental diseases are planned (Netwerk Kwaliteitsontwikkeling GGz). For some of these more rare diseases, the experiences with the CS for diabetes are even more useful, since implementing a similar trajectory with a governmentally funded action program would not be cost efficient for such diseases. Developing and publishing a CS does not automatically mean that it will be used in practice. The CS for diabetes has provided momentum for the further realization of the development and implementation of these Care Standards to improve the care for people with a chronic disease in the Netherlands. Moreover, from an international point of view, the Netherlands can be regarded as unique in the use of the Care Standard for diabetes. The results of this dissertation can therefore also inform similar future approaches in other countries.

\section{Planning}

The studies included in this dissertation have provided concrete leads to further optimize prior conditions needed to provide optimal care to patients.

As a result of the promising findings in the NAD pilot regions, a next step in the implementation of the CS for diabetes could be to implement the care on a local level, embedding it into a community approach which also provides opportunities for prevention (of other chronic diseases). Other partners, such as municipalities, municipal health services, health insurers, business, schools and sport clubs also play a role within this approach. When the NAD ended (June 2013), the NDF continued their activities related 
to the implementation and continuation of the CS and the next step on a local level through their expedition for sustainable care (Expeditie Duurzame Zorg). This expedition is a public-private partnership aimed to achieve sustainable care and a more vital society. This approach is also in line with the spearheads of the policy of the Dutch Ministry of Health, Welfare and Sport. In their most recent position paper, the ministry described that care and prevention should be provided close to people in their neighborhood which prescribes a community approach (Ministerie van Volksgezondheid Welzijn en Sport, 2011).

Another development is that of health care moving towards the provision of care fitting the individual needs and situations of patients. Currently, we see an international and national need to abandon the one-size-fits-all approach and move towards personalized care. In the Netherlands a group of experts and scientists is currently working on the development of so called 'patient profiles' in collaboration with the NDF. These profiles will be a new tool which can help health care professionals to provide personalized care, tailored to the needs of patients with diabetes. Within these profiles treatment-, personal- and environmental factors are taken into account when tailoring care and treatment. Currently three main profiles are developed for diabetes type 1, diabetes type 2 and obesity. Each profile describes two main issues: the intensity of the treatment and the need for professional support. An important objective of this personalized approach is to provide efficient care for the growing number of people with diabetes. The Netherlands is the first country worldwide that will start with evidence-based application of patient profiles.

Furthermore, preventive activities targeting chronic diseases need to become embedded in primary care and close collaboration with the public domain needs to be established within an integrated multidisciplinary approach. In 2012, prevention was added to the CS by means of an addendum. One of the most recent Dutch initiatives for collaboration in prevention of chronic diseases concerns the entry of the Netherlands Diabetes Federation into the National Prevention Program 'Alles is gezondheid'. This program aims to bring professionals and domains together to collaborate and provide care and prevention on a local level (Rijksoverheid, 2014).

Finally, the experiences with the implementation of the CS for diabetes underline the benefits that could be gained if diabetes care were to move towards an integrated multidisciplinary approach of chronic diseases worldwide. Although other chronic diseases are different in etiology, management and prognosis, the organisation and quality of care needs to meet the same criteria as the care for diabetes and disease management strategies are similar across most chronic diseases. Despite the international differences in health care systems, there is international consensus about the goals of treatment for diabetes and the same type and quality of care needs to be provided to patients with one or more chronic diseases (American Diabetes Association, 2013). Most approaches are however still disease-specific despite the often multiple health problems among people with chronic conditions (Nolte et al., 2012). The Care Standards and the 
bundled payment approach are useful instruments in organising integrated care. In the Netherlands, we have seen multiple collaborations established in the past years aimed at developing an integrated multidisciplinary approach to cope with the rising public health impact of chronic diseases. One of these collaborations concerns an agreement between the partnership Overweight Netherlands, the platform 'Vitale Vaten' ('Vital Veins') and the Netherlands Diabetes Federation. All three organizations focus on providing high quality care for people with (a high risk of) diabetes, cardiovascular diseases and obesity. The aim of this collaboration is to organize the care for these patient groups more effectively and efficiently, in order to increase the quality and patient centeredness of care. 


\section{References}

Algemene Rekenkamer (2010). Afstemming in de zorg rond chronische aandoeningen: terugblik 2010. Den Haag: Algemene Rekenkamer.

American Diabetes Association (2011). Standards of medical care in diabetes-2011. Diabetes care, 34 (Suppl 1), S11-61.

American Diabetes Association (2013). Standards of Medical Care in Diabetes-2013. Diabetes care, 136 (Suppl1), S11-S66.

Baan, C. A., Poos, M.J.J.C., Uiters, E., \& Savelkoul, M. (2014). Hoe vaak komt diabetes mellitus voor en hoeveel mensen sterven eraan? Volksgezondheid Toekomst Verkenning, Nationaal Kompas Volksgezondheid. Retrieved March 20, 2014 from http://www.nationaalkompas.nl/gezondheid-en-ziekte/ziekten-enaandoeningen/endocriene-voedings-en-stofwisselingsziekten-en-immuniteitsstoornissen/diabetesmellitus/omvang/

Baan, C. A., van Baal, P.H.M., Jacobs-van der Bruggen, M.A.M., Verkley, H., Poos, M.J.J.C., Hoogenveen, R.T.,\& Schoenmaker, C.G. (2009). Diabetes in Nederland: schatting van de huidige ziektelast en prognose voor 2025. Nederlands Tijdschrift voor Geneeskunde, 153(A580).

Baker, R., Camosso-Stefinovic, J., Gillies, C., Shaw, E.J., Cheater, F., Flottorp, S., \& Robertson, N. (2010).Tailored interventions to overcome identified barriers to change: effects on professional practice and health care outcomes (Review). Cochrane Database Systematic Reviews, 17(3), 1-32.

Barlow, J., Wright, C., Sheasby, J., Turner, A., \& Hainsworth, J. (2002). Self-management approaches for people with chronic conditions: a review. Patient Education and Counseling, 48(2), 177-187.

Barr, V.J., Robinson, S., Marin-Link, B., Underhill, L., Dotts, A., Ravensdale, D., \& Salivaras, S. (2003). The expanded Chronic Care Model: an integration of concepts and strategies from population health promotion and the Chronic Care Model. Hospital Quarterly, 7(1), 73-82.

Battersby, M.W., Ask, A., Reece, M.M., Markwick, M. J., \& Collins, J.P. (2003). The Partners in Health scale: The development and psychometric properties of a generic assessment scale for chronic condition selfmanagement. Australian Journal of Primary Health, 9(3), 41-52.

Beck, A.T. (1993). Cognitive therapy: nature and relation to behavior therapy. Journal of Psychotherapy Practice and Research, 2(4), 342-356.

Bekkering, G.E., Hendriks, H.J., van Tulder, M.W., Knol, D.L., Hoeijenbos, M., Oostendorp, R.A., \& Bouter, L.M. (2005). Effect on the process of care of an active strategy to implement clinical guidelines on physiotherapy for low back pain: a cluster randomised controlled trial. Quality \& Safety in Health Care, 14(2), 107-112.

Bodenheimer, T., Wagner, E.H., \& Grumbach, K. (2002a). Improving primary care for patients with chronic illness. JAMA-Journal of the American Medical Association, 288(14), 1775-1779.

Bodenheimer, T., Wagner, E.H., \& Grumbach, K. (2002b). Improving primary care for patients with chronic illness: the chronic care model, Part 2. JAMA-Journal of the American Medical Association, 288(15), 19091914. 
Branger, P.J., van 't Hooft, A., van der Wouden, J.C., Duisterhout, J. S., \& van Bemmel, J.H. (1998). Shared care for diabetes: supporting communication between primary and secondary care. Studies in Health Technology and Informatics, 52(Pt 1), 412-416.

Brez, S., Rowan, M., Malcolm, J., Izzi, S., Maranger, J., Liddy, C., Keely, E., \& Ooi, T.C. (2009). Transition from specialist to primary diabetes care: a qualitative study of perspectives of primary care physicians. BMC Family Practice, 10, 39.

Britt, E., Hudson, S.M., \& Blampied, N.M. (2004). Motivational interviewing in health settings: a review. Patient Education and Counseling, 53(2), 147-155.

Brom, L., Hopmans, W., Pasman, H.R., Timmermans, D.R., Widdershoven, G.A., \& Onwuteaka-Philipsen, B.D. (2014). Congruence between patients' preferred and perceived participation in medical decision-making: a review of the literature. BMC Medical Informatics and Decision Making, 14(1), 25.

Brown, J.B., Harris, S.B., Webster-Bogaert, S., Wetmore, S., Faulds, C., \& Stewart, M. (2002). The role of patient, physician and systemic factors in the management of type 2 diabetes mellitus. Family Practice, 19(4), 344349.

Brug, J., Spikmans, F., Aartsen, C., Breedveld, B., Bes, R., \& Fereira, I. (2007). Training dietitians in basic motivational interviewing skills results in changes in their counseling style and in lower saturated fat intakes in their patients. Journal of Nutrition Educaton and Behavior, 39(1), 8-12.

Brug, J., van Dale, D., Lanting, L., Kremers, S., Veenhof, C., Leurs, M., van Yperen, T., \& Kok, G. (2010). Towards evidence-based, quality-controlled health promotion: the Dutch recognition system for health promotion interventions. Health Education Research, 25(6), 1100-1106.

Cabana, M.D., Rand, C.S., Powe, N.R., Wu, A. W., Wilson, M.H., Abboud, P-A.C., \& Rubin, H.R. (1999). Why don't physicians follow clinical practice guidelines? JAMA-Journal of the American Medical Association, 282(15), 1458-1465.

Calsbeek, H., Ketelaar, N.A., Faber, M.J., Wensing, M., \& Braspenning, J. (2013). Performance measurements in diabetes care: the complex task of selecting quality indicators. International Journal for Quality in Health Care, 25(6), 704-709.

Carlsen, B., \& Bringedal, B. (2011). Attitudes to clinical guidelines-do GPs differ from other medical doctors? BMJ Quality and Safety, 20, 158-162.

Carroll, C., Patterson, M., Wood, S., Booth, A., Rick, J., \& Balain, S. (2007). A conceptual framework for implementation fidelity. Implementation Science, 2(40), 1-9.

Centrale Commissie Mensgebonden Onderzoek. Retrieved March 5, 2014, from http://www.ccmo.nl/nl/uwonderzoek-wmo-plichtig-of-niet

Chen, S.M., Creedy, D., Lin, H.S., \& Wollin, J. (2012). Effects of motivational interviewing intervention on selfmanagement, psychological and glycemic outcomes in type 2 diabetes: a randomized controlled trial. International Journal of Nursing Studies, 49(6), 637-644.

Chesover, D., Tudor-Miles, P., \& Hilton, S. (1991). Survey and audit of diabetes care in general practice in south London. British Journal of General Practice, 41(348), 282-285.

Coates, V.E., \& Boore, J.R. (1998). The influence of psychological factors on the self-management of insulindependent diabetes mellitus. Journal of Advanced Nursing, 27(3), 528-537.

Cochran, J., \& Conn, V.S. (2008). Meta-analysis of quality of life outcomes following diabetes self-management training. Diabetes Education, 34(5), 815-823.

Cochrane, L.J., Olson, C.A., Murray, S., Dupuis, M., Tooman, T., \& Hayes, S. (2007). Gaps between knowing and doing: Understanding and assessing the barriers to optimal health care. Journal of Continuing Education in the Health Professions, 27(2), 94-102.

Cohen, J. (1988). Statistical Power Analysis for the Behavioral Sciences. New York: Academic Press.

Coordinatieplatform Zorgstandaarden (2010). Zorgstandaarden in model. Rapport over het model voor zorgstandaarden bij chronische ziekten. Den Haag: ZonMw.

Cott, C.A., Gignac, M.A., \& Badley, E.M. (1999). Determinants of self rated health for Canadians with chronic disease and disability. Journal of Epidemiology \& Community Health, 53(11), 731-736. 
Damschroder, L.J., Aron, D.C., Keith, R.E., Kirsh, S.R., Alexander, J.A., \& Lowery, J.C. (2009). Fostering implementation of health services research findings into practice: a consolidated framework for advancing implementation science. Implementation Science, 4, 50.

Davies, P., Walker, A.E. \& Grimshaw, J.M. (2010). A systematic review of the use of theory in the design of guideline dissemination and implementation strategies and interpretation of the results of rigorous evaluations. Implementation Science, 5, 14.

De Bakker, D.H., Struijs, J.N., Baan, C.B., Raams, J., de Wildt, J.E., Vrijhoef, H.J., \& Schut, F.T. (2012). Early results from adoption of bundled payment for diabetes care in the Netherlands show improvement in care coordination. Health Affairs, 31(2), 426-433.

De Veer, A.J.E., Fleuren, M.A.H., Bekkema, N., \& Francke, A.L. (2011). Succesfull implementation of new technologies in nursing care: a questionnaire study of nurse-users. BMC Medical Informatics \& Decision Making, 11, 67 .

Deci, E.L., \& Ryan, R.M. (1985). The general causality orientations scale: Self-determination in personality. Journal of Research in Personality, 19(2), 109-134.

Deci, E.L., \& Ryan, R.M. (2008). Self-determination theory: A macrotheory of human motivation, development, and health. Canadian Psychology/Psychologie Canadienne, 49(3), 182-185.

Deci, E.L., \& Ryan, R.M. (2012). Self-determination theory in health care and its relations to motivational interviewing: a few comments. The International Journal of Behavioral Nutrition and Physical Activity, 9, 24.

Diabetes Vereniging Nederland. Diabetes Zorgwijzers. Retrieved March 25, 2014 from http://www.dvn.nl/downloads/diabetes-zorgwijzers

Dietrich, U.C. (1996). Factors influencing the attitudes held by women with type II diabetes: a qualitative study. Patient Education and Counseling, 29(1), 13-23.

Dijkstra, R.F., Braspenning, J.C., Uiters, E., van Ballegooie, E., \& Grol, R.T. (2000). Perceived barriers to the implementation of diabetes guidelines in hospitals in The Netherlands. Netherlands Journal of Medicine/NederlandsTtijdschrift voor Geneeskunde, 56(3), 80-85.

Dyck, L.R., Caron, A., \& Aron, D. (2006). Working on the positive emotional attractor through training in health care. Journal of Management Development, 25(7), 671-688.

EADV \& NAD (2012). Van indicatiestelling tot uitvoering. Een multidisciplinaire richtlijn over zelfcontrole van bloedglucosewaarden door mensen met diabetes. Utrecht: EADV.

Eklund, M., Erlandsson, L.K., \& Hagell, P. (2012). Psychometric properties of a Swedish version of the Pearlin Mastery Scale in people with mental illness and healthy people. Nordic Journal of Psychiatry, 66(6), 380388.

Elissen, A.M., Duimel-Peeters, I.G., Spreeuwenberg, C., Spreeuwenberg, M., \& Vrijhoef, H.J. (2012). Toward tailored disease management for type 2 diabetes. American Journal of Managed Care, 18(10), 619-630.

Elissen, A.M.J. (2013). Going beyond the 'grand mean'. Advancing disease management science and evidence. (Dissertation). Maastricht University, Maastricht.

Evans-Lacko, S., Jarrett, M., McCrone, P., \& Thornicroft, G. (2010). Facilitators and barriers to implementing clinical care pathways. BMC Health Services Research, 10, 82.

Fall, E., Roche, B., Izaute, M., Batisse, M., Tauveron, I., \& Chakroun, N. (2013). A brief psychological intervention to improve adherence in type 2 diabetes. Diabetes \& Metabolism, 39(5), 432-438.

Falzer, P.R. \& Garman, D.M. (2010). Contextual decision making and the implementation of clinical guidelines: an xxample from mental health. Academic Medicine, 85(3), 548-555.

Farquhar, C.M., Kofa, E.W., \& Slutsky, J.R. (2002). Clinicians' attitudes to clinical practice guidelines: a systematic review. Medical Journal of Australia, 177(9), 502-506.

Farquhar, C.M., Stryer, D., \& Slutsky, J. (2002). Translating research into practice: the future ahead. International Journal for Quality in Health Care, 14(3), 233-249.

Feste, C., \& Anderson, R.M. (1995). Empowerment: from philosophy to practice. Patient Education and Counseling, 26(1-3), 139-144.

Fleuren, M., Wiefferink, K., \& Paulussen T. (2004). Determinants of innovation within health care organizations: literature review and Delphi study. International Journal of Quality in Health Care, 16(2): 107-123. 
Fleuren, M., Keer, M., \& Paulussen, T. (2014). Adviesrapport meten van het gebruik van de JGZ-richtlijnen. Leiden: TNO.

Fleuren, M., Wiefferink, K., \& Paulussen, T. (2004). Determinants of innovation within health care organizations: literature review and Delphi study. International Journal for Quality in Health Care, 16(2), 107-123.

Francke, A.L., Smit, M.C., de Veer, A.J., \& Mistiaen, P. (2008). Factors influencing the implementation of clinical guidelines for health care professionals: a systematic meta-review. BMC Medical Informatics and Decision Making, 8, 38.

Fretheim, A., Schunemann, H.J., \& Oxman, A.D. (2006). Improving the use of research evidence in guideline development: 15. Disseminating and implementing guidelines. Health Research Policy and Systems, 4, 27.

Gao, J., Wang, J., Zheng, P., Haardorfer, R., Kegler, M.C., Zhu, Y., \& Fu, H. (2013). Effects of self-care, selfefficacy, social support on glycemic control in adults with type 2 diabetes. BMC Family Practice, 14, 66.

Glasgow, R.E., Peeples, M., \& Skovlund, S.E. (2008). Where is the patient in diabetes performance measures? The case for including patient-centered and self-management measures. Diabetes Care, 31(5), 1046-1050.

Glasgow, R.E., Ruggiero, L., Eakin, E.G., Dryfoos, J., \& Chobanian, L. (1997). Quality of life and associated characteristics in a large national sample of adults with diabetes. Diabetes Care, 20(4), 562-567.

Goderis, G., Borgermans, L., Mathieu, C., Van Den Broeke, C., Hannes, K., Heyrman, J., \& Grol, R. (2009). Barriers and facilitators to evidence based care of type 2 diabetes patients: experiences of general practitioners participating to a quality improvement program. Implementation Science, 4, 41.

Gravel, K., Légaré, F., \& Graham, I.D. (2006). Barriers and facilitators to implementing shared decision-making in clinical practice: a systematic review of health professionals' perceptions. Implementation Science, 1(1), 16.

Greenhalgh, T., Robert, G., Macfarlane, F., Bate, P., \& Kyriakidou, O. (2004). Diffusion of innovations in service organizations: systematic review and recommendations. Milbank Quarterly, 82(4), 581-629.

Groeneveld, I.F., Proper, K.I., Absalah, S., van der Beek, A.J., \& van Mechelen, W. (2011). An individually based lifestyle intervention for workers at risk for cardiovascular disease: a process evaluation. American Journal of Health Promotion, 25(6), 396-401.

Grol, R. (1997). Personal paper. Beliefs and evidence in changing clinical practice. BMJ, 315(7105), 418-421.

Grol, R., Cluzeau, F.A., \& Burgers, J.S. (2003). Clinical practice guidelines: towards better quality guidelines and increased international collaboration. British Journal of Cancer, 89(Suppl1), S4-8.

Grol, R., \& Grimshaw, J. (2003). From best evidence to best practice: effective implementation of change in patients' care. Lancet, 362(9391), 1225-1230.

Grol, R., \& Wensing, M. (2006). Effectieve implementatie: een model. In: Implementatie: effective verbetering van de patiëntenzorg. Maarssen: Elsevier Gezondheidszorg.

Gulliford, M.C., Naithani, S., \& Morgan, M. (2007). Continuity of care and intermediate outcomes of type 2 diabetes mellitus. Family Practice, 24(3), 245-251.

Hader, J.M., White, R., Lewis, S., Foreman, J.L.B., MacDonald, P.W., \& Thompson, L.G. (2007) Doctors' views of clinical practice guidelines: a qualitative exploration using innovation theory. Journal of Evaluation in Clinical Practice, 13(4), 601-606.

Haggerty, J.L., Reid, R.J., Freeman, G.K., Starfield, B.H., Adair, C.E., \& McKendry, R. (2003). Continuity of care: a multidisciplinary review. BMJ, 327, 1219-1221.

Handelsman, Y., Mechanick, J.I., Blonde, L., Grunberger, G., Bloomgarden, Z.T., Bray, G.A., Dagogo-Jack, S., Davidson, J.A., Einhorn, D., Ganda, O., Garber, A.J., Hirsch, I.B., Horton, E.S., Ismail-Beigi, F., Jellinger, P.S., Jones, K.L., Jovanovic, L., Lebovitz, H., Levy, P., Moghissi, E.S., Orzeck, E.A., Vinik, A.I., \& Wyne, K.L. (2011). American Association of Clinical Endocrinologists Medical Guidelines for clinical practice for developing a diabetes mellitus comprehensive care plan: executive summary. Endocrine Practice, 17(2), 287-302.

Heinrich, E., Candel, M.J., Schaper, N.C., \& de Vries, N K. (2010). Effect evaluation of a Motivational Interviewing based counselling strategy in diabetes care. Diabetes Research and Clinical Practice, 90(3), 270-278.

Heisler, M., Smith, D.M., Hayward, R.A., Krein, S.L., \& Kerr, E.A. (2003). How well do patients' assessments of their diabetes self-management correlate with actual glycemic control and receipt of recommended diabetes services? Diabetes Care, 26(3), 738-743. 
Helmink, J.H., Meis, J.J., de Weerdt, I., Visser, F.N., de Vries, N.K., \& Kremers, S.P. (2010). Development and implementation of a lifestyle intervention to promote physical activity and healthy diet in the Dutch general practice setting: the BeweegKuur programme. The International Journal of Behavioral Nutrition and Physical Activity, 7, 49.

Helmink, J.H.M., Raaijmakers, L.G.M., Rutten, G.M., de Vries, N.K., \& Kremers, S.P.J. (2013). Gecombineerde leefstijl interventies in Nederland: ervaringen uit de BeweegKuur. Tijdschrift voor Gezondheidswetenschappen, 91(2), 88-90.

Hendriks, A.-M., Jansen, M.W., Gubbels, J.S., de Vries, N.K., Paulussen, T., \& Kremers, S.P. (2013). Proposing a conceptual framework for integrated local public health policy, applied to childhood obesity-the behavior change ball. Implementation Science, 8(1), 46.

Hermans, M.P., Elisaf, M., Michel, G., Muls, E., Nobels, F., Vandenberghe, H., \& Brotons, C. (2013). Benchmarking is associated with improved quality of care in type 2 diabetes: the OPTIMISE randomized, controlled trial. Diabetes Care, 36(11), 3388-3395.

Hillen, M.A., van Vliet, L.M., de Haes, H.C.J.M., \& Smets, E.M.A. (2013). Developing and administering scripted video vignettes for experimental research of patient-provider communication. Patient Education and Counseling, 91(3), 295-309.

Hodgkin, P., \& Taylor, J. (2013). Power to the people: what will bring about the patient centred revolution? BMJ, 347, f6701.

Holland-Barkis, P., Forjuoh, S.N., Couchman, G.R., Chapen. C., Rascoe, T.G., \& Reis, M.D. (2006). Primary care physicians'awareness and adherence to cervical cancer screening guidelines in Texas. Preventive Medicine, 42(2), 140-145.

Holt, D.T., Helfrich, C.D., Hall, C.G. \& Weiner B.J. (2010). Are you ready? How health professionals can comprehensivley conceptualize readiness for change. Journal of General Internal Medicine 25(1), 50-55.

Hosper, K., Deutekom, M., \& Stronks, P.K. (2008). The effectiveness of "Exercise on Prescription" in stimulating physical activity among women in ethnic minority groups in the Netherlands: protocol for a randomized controlled trial. BMC Public Health, 8, 406.

Houweling, S.T., Kleefstra, N., van Hateren, K.J.J., Kooy, A., Groenier, K.H., ten Vergert, E., Meyboom-de Jong, B., \& Bilo, H.J.G. (2009). Diabetes specialist nurse as main care provider for patients with type 2 diabetes. Netherlands Journal of Medicine, 67(7), 279-284.

Hughes R. \& Huby M. (2002) The application of vignettes in social and nursing research. Journal of Advanced Nursing, 37(4), 382-386.

Huijg, J.M., van der Zouwe, N., Crone, M.R., Verheijden, M.W., Middelkoop, B.J.C., \& Gebhardt, W.M. (2014). Factors influencing the introduction of physical activity interventions in primary health care: a qualitative study. International Journal of Behavioral Medicine, epub ahead of print.

Inspectie voor de Gezondheidszorg (2012). Implementatie zorgstandaard Diabetes na vier jaar onvoldoende gevorderd. Meer bestuurskracht nodig van zorggroepen. Utrecht: Inspectie voor de Gezondheidszorg.

Institute of Medicine (2012). Accelerating Progress in Obesity Prevention. Solving the Weight of the Nation. Washington: Institute of Medicine.

Institute of Medicine (2001). Crossing the Quality Chasm: A New Health System for the 21st Century Washington, DC: National Academy Press.

International Diabetes Federation (2011). IDF Diabetes Atlas. Fifth edition: International Diabetes Federation.

International Diabetes Federation (2012). IDF Diabetes Atlas. Fifth edition, update 2012: International Diabetes Federation.

International Diabetes Federation (2013). IDF Diabetes Atlas. Sixth edition: International Diabetes Federation.

Jansen, D., \& Rijken, M. (2013). Evaluatie DVN pilotproject rond de implementatie van de Diabetes Zorgwijzer. Utrecht: NIVEL.

Jansink, R., Braspenning, J., Laurant, M., Keizer, E., Elwyn, G., Weijden, T., \& Grol, R. (2013). Minimal improvement of nurses' motivational interviewing skills in routine diabetes care one year after training: a cluster randomized trial. BMC Family Practice, 14, 44.

Kennisbank richtlijnontwikkeling (2014). Tool monitoring van richtlijnen. Retrieved, July 18th, 2014 from http://www.ha-ring.nl/tool-11\#h1-4-4-stel-vast-wat-er-geevalueerd-of-gemeten-moet-worden 
Keogh, K.M., Smith, S.M., White, P., McGilloway, S., Kelly, A., Gibney, J., \& O'Dowd, T. (2011). Psychological family intervention for poorly controlled type 2 diabetes. American Journal of Managed Care, 17(2), 105113.

Koelewijn-van Loon, M.S., van der Weijden, T., van Steenkiste, B., Ronda, G., Winkens, B., Severens, J.L., Wensing, M., Elwyn, G., \& Grol, R. (2009). Involving patients in cardiovascular risk management with nurse-led clinics: a cluster randomized controlled trial. Canadian Medical Association Journal, 181(12), E267-274.

Koshey, E., Koshey, V., \& Waterman, H. (2011). Action Research in Healthcare.London: Sage Publications Ltd.

Kripalani, S., LeFevre, F., Phillips, C.O., Williams, M.V., Basaviah, P., \& Baker, D.W. (2007). Deficits in communication and information transfer between hospital-based and primary care physicians: implications for patient safety and continuity of care. JAMA-Journal of the American Medical Association, 297(8), 831-841.

Kuijpers, W., Groen, W.G., Aaronson, N.K., \& van Harten, W.H. (2013). A systematic review of web-based interventions for patient empowerment and physical activity in chronic diseases: relevance for cancer survivors. Journal of Medical Internet Research, 15(2), e37.

Lamers, L.M., Stalmeier, P.F., McDonnell, J., Krabbe, P.F., \& van Busschbach, J.J. (2005). Kwaliteit van leven meten in economische evaluaties: het Nederlands EQ-5D-tarief. Nederlands Tijdschrift Geneeskunde, 149(28), 1574-1578.

Leemrijse, C.J., Ooms, L., \& Veenhof, C. (2009). Evaluatie van kansrijke beweegprogramma's om lichaamsbeweging in de bevolking te bevorderen Fase 2. Utrecht: NIVEL.

Legare, F., Ratte, S., Gravel, K., \& Graham, I.D. (2008). Barriers and facilitators to implementing shared decision-making in clinical practice: update of a systematic review of health professionals' perceptions. Patient Education and Counseling, 73(3), 526-535.

Legare, F., Ratte, S., Stacey, D., Kryworuchko, J., Gravel, K., Graham, I.D., \& Turcotte, S. (2010). Interventions for improving the adoption of shared decision making by healthcare professionals. Cochrane Database of Systematic Reviews, 12(5), 1-45.

Lemmens, L., de Jong-van Til, J., Struijs, J., Kooistra, M., \& Baan, C. (2012). Does the NDF Care Standard provide care groups enough support? (Biedt de NDF Zorgstandaard zorggroepen voldoende houvast?) Tijdschrift voor Gezondheidswetenschappen, 90(5), 295-302.

Lingard, L., Albert, M., \& Levinson, W. (2008). Grounded theory, mixed methods, and action research. BMJ, 337, a567.

Lugtenberg, M., Burgers, J.S., Han, D., \& Westert, G.P. (2014). General practitioners' preferences for interventions to improve guideline adherence. Journal of Evaluation in Clinical Practice, e-pub ahead of print.

Luijks, H., Schermer, T., Bor, H., van Weel, C., Lagro-Janssen, T., Biermans, M., \& de Grauw, W. (2012). Prevalence and incidence density rates of chronic comorbidity in type 2 diabetes patients: an exploratory cohort study. BMC Medicine, 10(1), 128.

Lundahl, B., Moleni, T., Burke, B.L., Butters, R., Tollefson, D., Butler, C., \& Rollnick, S. (2013). Motivational interviewing in medical care settings: A systematic review and meta-analysis of randomized controlled trials. Patient Education and Counseling, 93(2), 157-168.

Maddigan, S.L., Majumdar, S.R., \& Johnson, J.A. (2005). Understanding the complex associations between patient-provider relationships, self-care behaviours, and health-related quality of life in type 2 diabetes: a structural equation modeling approach. Quality of Life Research, 14(6), 1489-1500.

Maddigan, S.L., Majumdar, S.R., Toth, E.L., Feeny, D.H., \& Johnson, J.A. (2003). Health-related quality of life deficits associated with varying degrees of disease severity in type 2 diabetes. Health and Quality of Life Outcomes, 1(1), 78.

Makoul, G., \& Clayman, M.L. (2006). An integrative model of shared decision making in medical encounters. Patient Education and Counseling, 60(3), 301-312.

Malanda, U.L., Welschen, L.M., Riphagen, I.I., Dekker, J.M., Nijpels, G., \& Bot, S.D. (2012). Self-monitoring of blood glucose in patients with type 2 diabetes mellitus who are not using insulin. Cochrane Database of Systematic Reviews, 1, CD005060.

Mansour-Ghanaei, R., Joukar, F., Soati, F., \& Khanegha, A.G. (2013). Association between knowledge, locus of control and health belief with self-management, $\mathrm{Hb}$ A1c level and number of attendances in type 1 diabetes mellitus patients. International Journal of Clinical and Experimental Medicine, 6(6), 470-477. 
Marengoni, A., Angleman, S., Melis, R., Mangialasche, F., Karp, A., Garmen, A., Meinow, B., \& Fratiglioni, L. (2011). Aging with multimorbidity: a systematic review of the literature. Ageing Research Reviews, 10(4), 430-439.

Markland, D., Ryan, R.M., Tobin, V.J., \& Rollnick, S. (2005). Motivational Interviewing and Self-Determination Theory. Journal of Social \& Clinical Psychology, 24, 811-831.

Martens, M.K., Faassen, P., \& Hesselink, A.E. (2013a). Evaluatie implementatie NAD producten in vijf proefregio's. Haarlem: ResCon Research \& Consultancy.

Martens, M.K., Faassen, P., \& Hesselink, A.E. (2013b). Management summary: Evaluatie implementatie NAD producten in vijf proefregio's. Haarlem: ResCon Research \& Consultancy.

Martens, M., \& Hesselink, A. (2013c). Gebruikersonderzoek IZP. Haarlem: ResCon Research \& Consultancy.

Martinez-Gonzalez, N.A., Tandjung, R., Djalali, S., Huber-Geismann, F., Markun, S., \& Rosemann, T. (2014). Effects of physician-nurse substitution on clinical parameters: a systematic review and meta-analysis. PLOS One, 9(2), e89181.

Martins, R.K., \& McNeil, D.W. (2009). Review of Motivational Interviewing in promoting health behaviors. Clinical Psychology Review, 29(4), 283-293.

Mathus-Vliegen, L. (2006). De 'ideale' diëtist is flexibel en toegankelijk. Huisarts en diëtist, 61(5), 142-147.

May, C., Finch, T., Mair, F., Ballini, L., Dowrick, C., Eccles, M., Gask, L., MacFarlane, A., Murray, E., Rapley, T., Rogers, A., Treweek, S., Wallace, P., Anderson, G., Burns, J., \& Heaven, B. (2007). Understanding the implementation of complex interventions in health care: the normalization process model. BMC Health Services Research, 7, 148.

Mediquest. (2010). Diabetes Zorgmonitor. Analyserapport: onderzoek naar de kwaliteit van zorg voor mensen met diabetes. Utrecht: Mediquest.

Meis, J.J., Kremers, S.P., \& Bouman, M.P. (2012). Television Viewing Does Not Have to Be Sedentary: Motivation to Participate in a TV Exercise Program. Journal of Obesity, 752820.

Menec, V.H., \& Chipperfield, J.G. (1997). The interactive effect of perceived control and functional status on health and mortality among young-old and old-old adults. Journal of Gerontology series B: Psychology Sciences and Social Sciences, 52(3), 118-126.

Mertens, V., Bosma, H., Groffen D.A.I., \& van Eijk, J.T.M. (2012). Good friends, high income or resilience? What matters most for elderly patients? European Journal of Public Health, 22(5), 666-671.

Michie, S., Johnston, M., Abraham, C., Lawton, R., Parker, D., \& Walker, A. (2005). Making psychological theory useful for implementing evidence based practice: a consensus approach. Quality and Safety in Health Care, 14(1), 26-33.

Milder, I.E.J., Blokstra, A., de Groot, J., van Dulmen, S., Bemelmans, W.J.E. (2008). Lifestyle counseling in hypertension-related-visits- anylysis of video-taped general practice visits. BMC Family Practice, 9, 58.

Miller, W.R., \& Rollnick, S. (2002). Motivational Interviewing. Preparing people for change. New York: Guilford Press.

Miller, W.R., Yahne, C.E., Moyers, T.B., Martinez, J., \& Pirritano, M. (2004). A randomized trial of methods to help clinicians learn motivational interviewing. Journal of Consulting and Clinical Psychology, 72(6), 10501062.

Ministerie van Volksgezondheid Welzijn en Sport (2008). Programmatische aanpak van chronische ziekten. Den Haag: Ministerie van VWS.

Ministerie van Volksgezondheid Welzijn en Sport (2011). Gezondheid Dichtbij. Landelijke nota gezondheidsbeleid. Den Haag: Ministerie van VWS.

Moulding, N.T., Silagy, C.A. \& Weller, D.P. (1999). A framework for effective management of change in clinical practice: dissemination and implementation of clinical practice guidelines. Quality in Health Care, 8, 177183.

Nagelkerk, J., Reick, K., \& Meengs, L. (2006). Perceived barriers and effective strategies to diabetes selfmanagement. Journal of Advanced Nursing, 54(2), 151-158.

Nam, S., Chesla, C., Stotts, N.A., Kroon, L., \& Janson, S.L. (2010). Barriers to diabetes management: Patient and provider factors. Diabetes Research and Clinical Practice, 93, 1-9.

Nationaal Actieprogramma Diabetes (2011). Implementatieplan 2011-2012. Amersfoort: NAD. 
Nederlands Diabetes Federatie (2014). Update project Patiëntprofielen. Retrieved March 20, 2014, from http://www.diabetesfederatie.nl/nieuwsberichten/246-update-project-patientprofielen

Nederlands Huisartsen Genootschap (2013). NHG Standaard samenvattingskaart Diabetes Mellitus type 2. Retrieved March 20, 2014 from https://www.nhg.org/standaarden/samenvatting/diabetes-mellitus-type-2

Nederlandse Diabetes Federatie (2003). Zorgstandaard voor goede diabeteszorg. Een eerste voorwaarde voor een nieuw financieringsmodel. Retrieved March 20, 2014, from http://www.diabeteseemland.nl/protocollen/Zorgstandaard.pdf

Nederlandse Diabetes Federatie (2009). Nationaal Actieprogramma Diabetes Programmavoorstel. Amersfoort: NDF.

Nederlandse Diabetes Federatie (2013a). De NDF Zorgstandaard diabetes Type 1. Retrieved October 24, 2013, from http://www.zorgstandaarddiabetes.nl/type-1/

Nederlandse Diabetes Federatie (2013b). NDF Zorgstandaard Diabetes type 2. Retrieved October 24, 2013 from http://www.zorgstandaarddiabetes.nl/type-2/

Nederlandse Diabetes Federatie (2013c). NDF Zorgstandaard Diabetes. Addendum Geïndiceerde preventie van diabetes type 2. Retrieved December 6, 2013, from http://www.actieprogrammadiabetes.nl/images/stories/downloads/Preventie/addendum\%20preventie.pdf

Nederlandse Diabetes Federatie (2013d). Zorgstandaard diabetes.nl. Verschil Zorgstandaard, richtlijn en protocol. Retrieved December 6, 2013, from http://www.zorgstandaarddiabetes.nl/extrapage/verschilzorgstandaard-richtlijn-protocol/

Netwerk Kwaliteitsontwikkeling GGz. Retrieved July 20, 2014, from http://www.kwaliteitsontwikkelingggz.nl/

Krijgsman, J., de Bie, J., Burghouts, A., de Jong, J., Cath, G-J.,van Gennip, L., \& Friele, R. (2013). ehealth verder dan je denkt: ehealth monitor 2013. Den Haag: Nictiz \& Nivel.

Nijland, N., van Gemert-Pijnen, J.E., Kelders, S.M., Brandenburg, B.J., \& Seydel, E.R. (2011). Factors influencing the use of a Web-based application for supporting the self-care of patients with type 2 diabetes: a longitudinal study. Journal of Medical Internet Research, 13(3), e71.

Nobels, F., Debacker, N., Brotons, C., Elisaf, M., Hermans, M.P., Michel, G., \& Muls, E. (2011). Study rationale and design of OPTIMISE, a randomised controlled trial on the effect of benchmarking on quality of care in type 2 diabetes mellitus. Cardiovascular Diabetology, 10, 82.

Nolte, E., Knai, C., Hofmarcher, M., Conklin, A., Erler, A., Elissen, A., Flamm, M., Fullerton, B., Sonnichsen, A., \& Vrijhoef, H.J. (2012). Overcoming fragmentation in health care: chronic care in Austria, Germany and The Netherlands. Health Economics Policy and Law, 7(1), 125-146.

Noordman, J., De Vet, E., Van der Weijden, T., \& Van Dulmen, S. (2013a). Motivational interviewing within the different stages of change: An analysis of practice nurse-patient consultations aimed at promoting a healthier lifestyle. Social Science \&Medicine, 87, 60-67.

Noordman, J., Koopmans, B., Korevaar, J.C., Van der Weijden, T., \& Van Dulmen, S. (2013b). Exploring lifestyle counselling in routine primary care consultations: the professionals' role. Family Practice, 30(3), 332-340.

Norris, S.L., Engelgau, M.M., \& Venkat Narayan, K.M. (2001). Effectiveness of self-management training in type 2 diabetes: a systematic review of randomized controlled trials. Diabetes care, 24(3), 561-587.

O'Connor, P.J., Sperl-Hillen, J., Johnson, P.E., Rush, W.A., \& Crain, A.L. (2009). Customized feedback to patients and providers failed to improve safety or quality of diabetes care: a randomized trial. Diabetes Care, 32(7), 1158-1163.

Pajunen, P., Landgraf, R., Muylle, F., Neumann, A., Lindström, J., Schwarz, P., \& Peltonen, M. (2010). Quality and Outcome Indicators for Prevention of Type 2 Diabetes In Europe - IMAGE. Helsinki: National Institute for Health and Welfare.

Pantilat, S.Z., Lindenauer, P.K., Katz, P.P., \& Wachter, R.M. (2002). Primary care physician attitudes regarding communication with hospitalists. Disease-a-Month, 48(4), 218-229.

Patrick, H., \& Williams, G.C. (2012). Self-determination theory: its application to health behavior and complementarity with motivational interviewing. The International Journal of Behavioral Nutrition and Pysical Activity, 9, 18. 
Peabody, J.W., Luck, J., Glassman, P., Jain, S., Hansen, J., Spell, M., \& Lee, M. (2004). Measuring the quality of physician practice by using clinical vignettes: A prospective validation study. Annals of Internal Medicine, 141(10), 771-781.

Peabody, J.W., Luck, J., Glassman, P., Dresselhaus, T.R. , \& Lee, M. (2000). Comparison of vignettes, standardized patients, and chart abstraction: a prospective validation study of 3 methods for measuring quality. JAMA-Journal of the American Medical Association, 283(13), 1715-1722.

Pearlin, L.I., Nguyen, K.B., Schieman, S., \& Milkie, M.A. (2007). The life-course origins of mastery among older people. Journal of Health and Social Behavior, 48(2), 164-179.

Pearlin, L.I., \& Schooler, C. (1978). The structure of coping. Journal of Health and Social Behavior, 19(1), 2-21.

Pettiegrew, A.M., Woodman, R.W. \& Cameron, K.S. (2001). Studying organizational change and development: Challenges for future research. Journal of Academy of Medical Sciences, 44, 697-713.

Peyrot, M., \& Rubin, R.R. (2007). Behavioral and psychosocial interventions in diabetes. A conceptual review. Diabetes Care, 30(10), 2433-2440.

Ploeg, M. (2011). De patiënt als zorgverbeteraar. Medisch Contact, 17, 1089-1091.

Pollak, K.I., Alexander, S.C., Tulsky, J.A., Lyna, P., Coffman, C.J., Dolor, R.J., Gulbrandsen, P., \& Ostbye, T. (2011). Physician empathy and listening: associations with patient satisfaction and autonomy. Journal of American Board on Family Medicine, 24(6), 666-674.

Puder, J.J., \& Keller, U. (2003). Quality of diabetes care: problem of patient or doctor adherence? Swiss Medical Weekly, 133(39-40), 530-534.

Raad voor de Volksgezondheid en Zorg (2011). Het Chronic Care Model in Nederland. Den Haag: RVZ.

Raaijmakers, L.G.M., Martens, M.K., Bagchus, C., de Vries, N.K., \& Kremers, S.P.J. (2013). Perceptions of Dutch health care professionals regarding the Care Standard for diabetes. BMC Research Notes, 17(6), 417.

Raaijmakers, L.G.M., Hamers, F.J.M., Martens, M.K., Bagchus, C., de Vries, N.K., \& Kremers, S.P.J. (2013). Perceived facilitators and barriers in diabetes care: a qualitative study among health care professionals in the Netherlands. BMC Family Practice, 14(1), 114.

Raaijmakers, L.G. M., Martens, M.K., Bagchus, L., Jonkers, R., \& Kremers, S.P.J. (2010). NAD monitor 2010. Onderzoek onder zorgprofessionals en diabetespatiënten [in Dutch. Extensive summary in English available]. Maastricht: Universiteit Maastricht.

Raaijmakers, L. G.M., Martens, M.K., Hesselink, A.E., \& Kremers, S P.J. (2013). NAD monitor 2013. Onderzoek onder zorgprofessionals en diabetespatiënten [in Dutch. Extensive summary in English available]. Maastricht: Universiteit Maastricht.

Raaijmakers, L.G.M. M., Martens, M.K., Hesselink, A.E., De Weerdt, I., \& Kremers, S.P.J. (2013). The implementation of the Netherlands Diabetes Federation Care Standard for Diabetes anno 2013. Nederlands Tijdschrift voor Diabetologie. Available at http://www.rescon.nl/wp-content/uploads//2011/10/Raaijmakerset-al-implementation-CS-diabetes.pdf

Rabin, R., \& Charro, F. (2001). EQ5D: a measure of health status from the EuroQol group. Annuals of Medicine, 33(5).

Redekop, W.K., Koopmanschap, M.A., Stolk, R.P., Rutten, G.E., Wolffenbuttel, B.H., \& Niessen, L.W. (2002). Health-related quality of life and treatment satisfaction in Dutch patients with type 2 diabetes. Diabetes Care, 25(3), 458-463.

Reed, J.A., Ashton, H., Hollinghurst, S., \& Higgs, E.R. (2003). Diabetes self management: how are we doing? Practical Diabetes International, 20(9), 318-322.

Reeves, S., Fox, A., \& Hodges, B.D. (2009). The competency movement in the health professions: ensuring consistent standards or reproducing conventional domains of practice? Advances in Health Sciences Education, 14(4), 451-453.

Reid, R., Haggerty, J.L., \& McKendry, R. (2002). Defusing the Confusion: Concept and Measures of Continuity of Healthcare. Ottawa: Canadian Health Services Research Foundation.

Resnicow, K., \& McMaster, F. (2012). Motivational Interviewing: moving from why to how with autonomy support. The International Journal of Behavioral Nutrition and Physical Activity, 9, 19.

Rijksinstituut voor Volksgezondheid en Milieu (2007). Diabetes in Nederland. Omvang, risicofactoren en gevolgen, nu en in de toekomst. Bilthoven: RIVM. 
Rijksinstituut voor Volksgezondheid en Milieu (2011). Nederland de maat genomen 2009-2012. Monitoring van risicofactoren in de algemene bevolking. Bilthoven: RIVM.

Rijksoverheid (2014). Alles is gezondheid. Het Nationaal Programma Preventie 2014-2016. Retrieved March 20, 2014 from http://www.allesisgezondheid.nl/

Rogers, E.M. (2003). Diffusion of innovations. New York: Free Press.

Rose, E.A., Parfitt, G., \& Williams, S. (2005). Exercise causality orientations, behavioural regulation for exercise and stage of change for exercise: exploring their relationships. Psychology of Sport and Exercise, 6(4), 399414.

Rotter, J.B. (1966). Generalized expectancies for internal versus external control of reinforcement. Psychological Monographs, 80(1), 1-28.

Rubak, S., Sandbaek, A., Lauritzen, T., \& Christensen, B. (2005). Motivational interviewing: a systematic review and meta-analysis. British Journal of General Practice, 55(513), 305-312.

Rubin, R.R., \& Peyrot, M. (1999). Quality of life and diabetes. Diabetes/Metabolism Research and Reviews, 15(3), 205-218.

Rutten, G.E.H.M. (2014). Patiëntprofielen en zorg op maat. Paper presented at the Nationale Diabetes Dag 2014, Den Haag.

Rutten, G., Harting, J., Rutten, S.T.J., Bekkering, G.E., \& Kremers, S.P.J. (2009). Measuring physiotherapists' guideline adherence by means of clinical vignettes: a validation study. Journal of Evaluation in Clinical Practice, 12(5), 491-500

Ryan, R.M., \& Deci, E.L. (2000). Self-determination theory and the facilitation of intrinsic motivation, social development, and well-being. American Psychologist, 55(1), 68-78.

Sachs, M. (2006). Successful strategies and methods of nursing standards implementation. [Article in German] Pflege, 19, 33-44.

Samoocha, D., Bruinvels, D.J., Elbers, N.A., Anema, J.R., \& van der Beek, A.J. (2010). Effectiveness of web-based interventions on patient empowerment: a systematic review and meta-analysis. Journal of Medical Internet Research, 12(2), e23.

Sasseville, N., Simard, P., \& Mucha, J. (2012). An Integrated Approach for Chronic Disease Prevention. Retrieved March 20, 2014 from http://bchealthycommunities.ca/res/download.php?id=432

Schieman, S., \& Turner, H.A. (1998). Age, disability, and the sense of mastery. Journal of Health and Social Behavior, 39(3), 169-186.

Schipper, K., \& Abma, T.A. (2011). Coping, family and mastery: top priorities for social science research by patients with chronic kidney disease. Nephrology Dialysis Transplantation, 26(10), 3189-3195.

Schipper, K., Widdershoven, G.A., \& Abma, T.A. (2011). Citizenship and autonomy in acquired brain injury. Nursing Ethics, 18(4), 526-536.

Schoen, C., Osborn, R., Doty, M.M., Squires, D., Peugh, J., \& Applebaum, S. (2009). A survey of primary care physicians in eleven countries, 2009: perspectives on care, costs, and experiences. Health Affairs, 28(6), w1171-w1183.

Seidell, J.C., Halberstadt, J., Noordam, H., \& Niemer, S. (2012). An integrated health care standard for the management and prevention of obesity in The Netherlands. Family Practice, 29, 153-156.

Senecal, C., Nouwen, A., \& White, D. (2000). Motivation and dietary self-care in adults with diabetes: are selfefficacy and autonomous self-regulation complementary or competing constructs? Health Psychology, 19(5), 452-457.

Shigaki, C., Kruse, R.L., Mehr, D., Sheldon, K.M., Bin, G., Moore, C., \& Lemaster, J. (2010). Motivation and diabetes self-management. Chronic Illness, 6(3), 202-214.

Sinnige, J., Braspenning, J., Schellevis, F., Stirbu-Wagner, I., Westert, G., \& Korevaar, J. (2013). The prevalence of disease clusters in older adults with multiple chronic diseases--a systematic literature review. PLoS One, 8(11), 1-12.

Skaff, M.M.K., Mullan, J.T., Fisher, L., \& Chesla, C.A. (2010). A contextual model of control beliefs, behavior, and health: Latino and European Americans with type 2 diabetes. Psychology \& Health, 18(3), 295-312.

Sloan, F.A., Padron, N.A., \& Platt, A C. (2009). Preferences, beliefs, and self-management of diabetes. Health Services Research, 44(3), 1068-1087. 
Sluiter, A., van Wijland, H., Arntzenius, A., Bots, A., Dijkhorst-Oei, L., van der Does, F., Palmen, J., Potter van Loon, B., Schaper, N.C., \& van Balen, J. (2012). Landelijke Transmurale Afspraak Diabetes Mellitus Type 2. Huisarts \& Wetenschap, 55(1), S1-S12.

Snoek, F.J. (2000). Quality of life: a closer look at measuring patients' well-being. Diabetes Spectrum, 13(1), 2428.

Solberg, P.A., Halvari, H., \& Ommundsen, Y. (2013). Linking exercise and causality orientations to change in well-being among older adults: does change in motivational variables play a role? Journal of Applied Social Psychology, 43(6), 1259-1272.

Spijkerman, A.M.W., \& Baan, C.A. (2014). Diabetes mellitus: Hoe zijn preventie en zorg georganiseerd? Volksgezondheid Toekomst Verkenning, Nationaal Kompas Volksgezondheid. Retrieved March 20, 2014 from http://www.nationaalkompas.nl/gezondheid-en-ziekte/ziekten-en-aandoeningen/endocriene-voedingsen-stofwisselingsziekten-en-immuniteitsstoornissen/diabetes-mellitus/diabetes-mellitus-preventie-enzorg/

Spijkerman, A.M.W., Baan, C.A., \& Eysink, P.E.D. (2013). Wat is diabetes mellitus en wat is het beloop. Volksgezondheid Toekomst Verkenning, Nationaal Kompas Volksgezondheid. Retrieved, December 6, 2013, from http://www.nationaalkompas.nl/gezondheid-en-ziekte/ziekten-en-aandoeningen/endocriene-voedingsen-stofwisselingsziekten-en-immuniteitsstoornissen/diabetes-mellitus/beschrijving/

Sport en Bewegen in de buurt. (2014). Retrieved March 20, 2014, from http://www.sportindebuurt.nl/

Stiggelbout, A.M., Van der Weijden, T., De Wit, M., Frosch, D., Légaré, F., Montori, V.M., Trevena, L., \& Elwyn, G. (2012). Shared decision making: really putting patients at the centre of healthcare. BMJ, 344.

Struijs, J.N., \& Baan, C.A. (2011). Integrating care through bundled payments--lessons from The Netherlands. New England Journal of Medicine, 364(11), 990-991

Struijs, J.N., van Til, J.T., \& Baan, C.A. (2010). Experimenting with a bundled payment system for diabetes care in the Netherlands. The first tangible effects. Bilthoven: Centrum voor Preventie- en Zorgonderzoek.

Sundaram, M., Kavookjian, J., Patrick, J.H., Miller, L.-A., Madhavan, S.S., \& Scott, V.G. (2007). Quality of life, health status and clinical outcomes in Type 2 diabetes patients. Quality of Life Research, 16(2), 165-177.

Tabak, R.G., Khoong, E.C., Chambers, D.A., \& Brownson, R.C. (2012). Bridging research and practice: models for dissemination and implementation research. American Journal of Preventive Medicine, 43(3), 337-350.

Teixeira, P.J., Carraça, E.V., Markland, D., Silva, M.N., \& Ryan, R.M. (2012). Exercise, physical activity, and selfdetermination theory: A systematic review. The International Journal of Behavioral Nutrition and Physical Activity, 9(1), 78.

The EuroQol Group. (1990). EuroQol-a new facility for the measurement of health-related quality of life. Health Policy, 16(3), 199-208.

Tiemeier, H., de Vries, W.J., van het Loo, M., Kahan, J.P., Klazinga, N., Grol, R. \& Rigter, H. (2002). Guideline adherence rates and interprofessionalvariation in a vignette study of depression. Quality and Safety in Health Care, 11(3), 214-218.

Tol, J., Swinkels, I.C., Struijs, J.N., Veenhof, C., \& de Bakker, D.H. (2013). Integrating care by implementation of bundled payments: results from a national survey on the experience of Dutch dietitians. International Journal of Integrated Care, 13, 1-18.

Toobert, D.J., Hampson, S.E., \& Glasgow, R.E., (2000). The summary of diabetes self-care activities measure. Diabetes Care, 23, 943-950.

Towle, A., \& Godolphin, W. (1999). Framework for teaching and learning informed shared decision making. BMJ, 319(7212), 766.

Tsiachristas, A., Hipple-Walters, B., Lemmens, K.M., Nieboer, A.P., \& Rutten-van Molken, M.P. (2011). Towards integrated care for chronic conditions: Dutch policy developments to overcome the (financial) barriers. Health Policy, 101(2), 122-132.

Ubink-Veltmaat, L J., Bilo, H.J., Groenier, K.H., Rischen, R.O., \& Meyboom-de Jong, B. (2005). Shared care with task delegation to nurses for type 2 diabetes: prospective observational study. Netherlands Journal of Medicine, 63(3), 103-110.

Uijen, A.A., \& van de Lisdonk, E.H. (2008). Multimorbidity in primary care: prevalence and trend over the last 20 years. European Journal of General Practice, 14 Suppl 1, 28-32. 
Ujcic-Voortman, J.K., Schram, M.T., Jacobs-van der Bruggen, M.A., Verhoeff, A.P., \& Baan, C.A. (2009). Diabetes prevalence and risk factors among ethnic minorities. The European Journal of Public Health, 19(5), 511515.

Vahdat, S., Hamzehgardeshi, L., Hessam, S., \& Hamzehgardeshi, Z. (2014). Patient involvement in health care decision making: a review. IranianRed Crescent Medical Journal, 16(1), 1-7.

Van Bruggen, R., Gorter, K.J., Stolk, R.P., Verhoeven, R.P., \& Rutten, G.E. (2008). Implementation of locally adapted guidelines on type 2 diabetes. Family Practice, 25(6), 430-437.

Van der Wees, P.J., Jamtvedt, G., Rebbeck, T., De Bie, R.A., Dekker, J., \& Hendriks, E.J. (2008). Multifaceted strategies may increase implementation of physiotherapy clinical guidelines: a systematic review. Australian Journal of Psychology, 54(4), 233-41.

Van Dijk, C.E., \& Korevaar, J.C. (2011). Integrale bekostiging: eerste ervaringen na één jaar invoering. Utrecht: NIVEL.

Van Dillen, S.M., Noordman, J., van Dulmen, S., \& Hiddink, G.J. (2014). Examining the content of weight, nutrition and physical activity advices provided by Dutch practice nurses in primary care: analysis of videotaped consultations. European Journal of Clinical Nutrition, 68(1), 50-56.

Van Dillen, S.M., van Binsbergen, J.J., Koelen, M.A., \& Hiddink, G.J. (2013). Nutrition and physical activity guidance practices in general practice: a critical review. Patient Education and Counseling, 90(2), 155-169.

Van Til, J. T., de Wildt, E., \& Struijs, J.N.,(2010). De organisatie van zorggroepen anno 2010: Huidige stand van zaken en de ontwikkelingen in de afgelopen jaren. Bilthoven: RIVM.

Vansteenkiste, M., \& Sheldon, K.M. (2006). There's nothing more practical than a good theory: integrating motivational interviewing and self-determination theory. British Journal of Clinical Psychology, 45(Pt 1), 63-82.

Vansteenkiste, M., Williams, G.C., \& Resnicow, K. (2012). Toward systematic integration between SelfDetermination Theory and Motivational Interviewing as examples of top-down and bottom-up intervention development: Autonomy or volition as a fundamental theoretical principle. International Journal of Behavioral Nutrition and Physical Activity, 9(23).

Verweij, L.M., Proper, K., Leffelaar, E.R., Weel, A., Nauta, A.P., Hulshof, C., \& van Mechelen, W. (2012). Barriers and facilitators to implementation of an occupational health guideline aimed at preventing weight gain among employees in the Netherlands. Journal of Occupational and Environmental Medicine, 54(8), 954960.

Verweij, A. (2008). Scholing en opleiding: indeling opleidingsniveau. Bilthoven: RIVM.

Vrijhoef, H.J., Diederiks, J.P., Spreeuwenberg, C., \& Wolffenbuttel, B H. (2001). Substitution model with central role for nurse specialist is justified in the care for stable type 2 diabetic outpatients. Journal of Advanced Nursing, 36(4), 546-555.

Wantland, D.J., Portillo, C J., Holzemer, W L., Slaughter, R., \& McGhee, E.M. (2004). The effectiveness of Webbased vs. non-Web-based interventions: a meta-analysis of behavioral change outcomes. Journal of Medical Internet Research, 6(4), e40.

Watkins, K.W., Connell, C.M., Fitzgerald, J.T., Klem, L., Hickey, T., \& Ingersoll-Dayton, B. (2000). Effect of adults' self-regulation of diabetes on quality-of-life outcomes. Diabetes Care, 23(10), 1511-1515.

Wens, J., Vermeire, E., van Royen, P, Sabbe, B. \& Denekens, J. (2005). GP's perspectives of type 2 diabetes patients' adherence to treatment: A qualitative analysis of barriers and solutions. BMC Family Practice, 6(20).

Whittemore, R., Melkus, G., Wagner, J., Dziura, J., Northrup, V., \& Grey, M. (2009). Translating the diabetes prevention program to primary care: a pilot study. Nursing Research, 58(1), 2-12.

Williams, G.C., Freedman, Z.R., \& Deci, E.L. (1998). Supporting autonomy to motivate patients with diabetes for glucose control. Diabetes Care, 21(10), 1644-1651.

Williams, G.C., Gagné, M., Mushlin, A.I., \& Deci, E.L. (2002). Motivation for behavior change in patients with chest pain. Health Education Research, 105, 304-321.

Williams, G.C., Gagne, M., Ryan, R.M., \& Deci, E.L. (2002). Facilitating autonomous motivation for smoking cessation. Health psychology : official journal of the Division of Health Psychology, American Psychological Association, 21(1), 40-50. 
Williams, G.C., Grow, V.M., Freedman, Z R., Ryan, R.M., \& Deci, E.L. (1996). Motivational predictors of weight loss and weight-loss maintenance. Journal of Personality and Social Psychology, 70(1), 115-126.

Williams, G.C., Lynch, M., \& Glasgow, R.E. (2007). Computer-assisted intervention improves patient-centered diabetes care by increasing autonomy support. Health Psychology, 26(6), 728.

Williams, G.C., McGregor, H.A., King, D., Nelson, C.C., \& Glasgow, R.E. (2005). Variation in perceived competence, glycemic control, and patient satisfaction: relationship to autonomy support from physicians. $\mathrm{Pa}$ tient Education and Counseling, 57(1), 39-45.

Williams, G.C., McGregor, H.A., Zeldman, A., Freedman, Z.R., \& Deci, E.L. (2004). Testing a self-determination theory process model for promoting glycemic control through diabetes self-management. Health Psychology, 23(1), 58-66.

Williams, G.C., Patrick, H., Niemiec, C.P., Williams, L.K., Divine, G., Lafata, J.E., Heisler, M., Tunceli, K., \& Pladevall, M. (2009). Reducing the health risks of diabetes: how self-determination theory may help improve medication adherence and quality of life. Diabetes Education, 35(3), 484-492.

Williams, G.C., Rodin, G.C., Ryan, R.M., Grolnick, W.S., \& Deci, E L. (1998). Autonomous regulation: the motivational basis of adherence to medical regimens. Health Psychology, 17, 269-276.

Williams, K., \& Bond, M. (2002). The roles of self-efficacy, outcome expectancies and social support in the selfcare behaviours of diabetics. Psychology, Health \& Medicine, 7(2), 127-141.

Williamson, J.C., Glauser, T.A., Holder, P., Schneuder, D., Kruger, D.F., Urquhart, B.S., Whitfield, S.F., \& Dubois, A.M. (2013). Current Practice Patterns and Identified Educational Needs of Health Care Providers in Managing Patients with type 2 diabetes. Clinical Diabetes, 31(1), 3-9.

World Health Organization. (2011). BMI classification. Retrieved May 21, 2013, from http://apps.who.int/bmi/index.jsp?introPage=intro_3.html

World Health Organization. (2013). Factsheet diabetes. Retrieved May 21, 2013, from http://www.who.int/mediacentre/factsheets/fs312/en/index.html

World Health Organization. (2014). Integrated chronic disease prevention and control. Retrieved March 20, 2014, from http://www.who.int/chp/about/integrated_cd/en/index2.html

Zorginstituut Nederland. Retrieved July 20, 2014, from http://www.zorginstituutnederland.nl/ 



\section{Summary}

Diabetes mellitus is a rapidly growing health problem, which affects approximately 382 million people worldwide. In the Netherlands, 834,100 people were diagnosed with diabetes on January 1st, 2011 and this number increased by 87,000 patients in that same year. Diabetes is a multifactorial health problem that requires a multidisciplinary approach to prevention and treatment. As in most industrialized countries, Dutch health care faces the challenge of guaranteeing continuity and quality of care for the growing number of people with diabetes. For this reason multiple changes in diabetes care have been introduced in recent years; one of these concerns the introduction and implementation of the Netherlands Diabetes Federation (NDF) Care Standard (CS) for diabetes. The CS describes the norm for high quality diabetes care and functions as a general overarching framework for the guidelines for each individual professional group and focuses on a multidisciplinary approach to diabetes care and prevention. The Netherlands can be regarded as unique in the use of the CS for diabetes. In 2009, the NDF started the National Action program Diabetes (NAD) (2009-2013) with funding of the Dutch Ministry of Health, Welfare and Sport. The main objective of the NAD was the systematic nationwide implementation of the CS (Nederlandse Diabetes Federatie, 2009).

The aim of this dissertation was to provide a closer look at diabetes care and the introduction of the CS for diabetes from the perspectives of both health care professionals and diabetes patients. From the perspective of health care professionals, this dissertation assessed their perceptions regarding the content and implementation of the CS and perceived facilitating and impeding factors in diabetes care. From the perspective of patients, this dissertation examined how Self-Determination Theory concepts and mastery were associated with Dutch type 1 and type 2 diabetes patients' self-management activities, general diabetes control and health-related quality of life.

After a general introduction in chapter 1, two studies from the perspective of health care professionals (chapter 2 and 3) will be described, followed by two studies from the per- 
spective of diabetes patients (chapter 4 and 5). In chapter 6 and 7 studies from an integration of both the perspectives of health care professionals and patients are presented. The dissertation ends with a general discussion in chapter 8.

\section{Perspective of health care professionals}

Chapter 2 describes a cross-sectional questionnaire study that assessed the perceptions of Dutch health care professionals $(\mathrm{N}=1547)$ regarding the CS for diabetes and barriers to using it. A limited percentage of health care professionals had the CS in possession. Almost two-thirds of the professionals who were to some extent familiar with the CS thought it contributed greatly to ensuring the quality of care, and the feasibility of working in accordance with the CS was largely endorsed. However, professionals tended to perceive the guidelines issued by the own professional association as the norm for high quality diabetes care, rather than the CS. A minority of the professionals who were to some extent familiar with the CS described themselves as working in complete accordance with the CS. The main barrier to using the CS was the lack of effective lifestyle interventions (or access to them) to provide care for people with diabetes or those at increased risk for the disease. Another important perceived barrier was that of financial, legislative and regulations-related issues regarding care and prevention in accordance with the CS. Attention should be given to these barriers to facilitate adherence to the CS.

Chapter 3 presents a qualitative study in which 18 health care professionals were interviewed in order to identify perceived facilitators and barriers in diabetes care using the Chronic Care Model (CCM). One of the major facilitators was the more prominent role of the practice nurses and diabetes nurses in diabetes care as a result of substitution of care. Other reported facilitators were the CS itself and multidisciplinary collaboration, although there is still room for improvement in collaboration with certain professional groups, i.e. dieticians, physical therapists and pharmacists, and between professionals working in primary and secondary care. Furthermore, benchmarking was perceived as a facilitating factor, while the majority reported the quality of the indicators for benchmarking to be a barrier. Major barriers in the health care system were the bundled payment system for the funding of diabetes care, the role of the health insurers and the lack of motivation on the part of patients. Another important barrier was the lack of awareness of lifestyle programs and prevention initiatives for diabetes patients among professionals, which indicated the usefulness of the Expanded CCM.

\section{Perspective of diabetes patients}

Chapter 4 describes a cross-sectional questionnaire study that examined how Dutch type $1(\mathrm{~N}=143)$ and type $2(\mathrm{~N}=384)$ diabetes patients' perceived autonomy support, as well as their perceived competence and treatment self-regulation, are associated with their 
diabetes self-care activities (healthy diet, physical activity, monitoring blood glucose, medication use) and general diabetes control. Most participants felt competent, supported in their autonomy, and perceived themselves to autonomously self-regulate their diabetes. The results underlined the importance of perceived competence in type 1 and type 2 diabetics, as this was strongly associated with adhering to a healthy diet and general diabetes control. Our findings also emphasized the need for autonomy supportive health care professionals in diabetes care. Interestingly, perceived competence appeared to mediate the influence of autonomy support on general diabetes control, especially in type 1 diabetics, while in type 2 diabetics' autonomy support remains to have a direct association with general diabetes control.

Chapter 5 presents a cross sectional questionnaire study among 3352 patients with type 2 diabetes. The aim of this study was to assess the influence of patients' mastery and perceived autonomy support from their primary diabetes care provider on their selfmanagement skills and health-related quality of life. Dutch type 2 diabetes patients were found to have relatively high scores for perceived autonomy support and selfmanagement, and to perceive a relatively low sense of mastery. Patients with more physical or psychological complications had significantly lower scores on mastery, perceived autonomy support, self-management and HRQOL, which is in line with our hypotheses. Our findings emphasized and confirmed the need for an autonomy-supportive counseling style by diabetes care providers. Furthermore, our results underlined the importance of mastery in relation to patients' perceived autonomy support from their primary caregiver, self-management skills and HRQOL.

\section{An integration of the perspectives of health care professionals and patients}

Chapter 6 describes two cross-sectional studies conducted in 2010 and 2013 among health care professionals and type 1 and type 2 diabetes patients. The main aim of these studies was to assess professionals' familiarity with and appreciation of the CS for diabetes and the quality of the implementation of the CS. Moreover, this chapter aimed to compare the results of both studies. In 2013, health care professionals were significantly more often familiar with the CS compared to 2010 and perceived themselves more often working completely in accordance with the CS. The majority had a positive attitude towards the CS and its contribution to ensuring the quality of diabetes care. Overall, diabetes patients were satisfied with their contact with their caregiver(s) and perceived a high degree of involvement in their treatment. As the CS for diabetes was the first one to be produced, experiences with the implementation of this standard can be used in the implementation of other Care Standards. A next step could be to implement care at a local level, and to embed it into a community approach thereby also focusing on the prevention of other chronic diseases. 
Chapter 7 aimed to provide insight in the implementation of the Netherlands Action program Diabetes (NAD) over the past decade, with the introduction of the CS as main aim, and including a specific implementation plan and piloting in several regions. This program and the introduction of the CS can function as an example for similar approaches in other countries. Overall, positive changes in diabetes care were detected in the period 2010 - 2013. A comparison of the results in specific pilot regions with the rest of the country revealed that professionals in these regions scored significantly more positive on implementation and appreciation of the CS. This positive trend was reflected by the high levels of reported patient satisfaction and involvement in treatment. Professionals who were in possession of the CS had significantly better scores on the implementation of several elements of the CS than HCPs who were not in possession of the CS. In retrospect the CS has provided momentum for the realization of various processes relating to the implementation of standards to improve the care for people with a chronic disease in the Netherlands. Experiences with the NAD and the CS underline the need to move towards an integrated multidisciplinary approach of diabetes care worldwide.

Chapter 8 presents a summary of and reflection on the main findings of the studies included in this dissertation. It also presents methodological considerations which concern issues related to the study design, study population, measurement instruments and statistical analyses; and the strengths of the studies. The chapter then presents implications of the findings for practice, theory and further research. The main conclusion is that the NAD has resulted in the CS to become embedded in the Dutch health care system. The introduction of the CS, supported by the Naional Diabetes Action Program, and the bundled payment approach as a parallel development, has provided momentum for the realization of various facilitating processes relating to the wider implementation of standards to improve the care for people with other chronic diseases in the Netherlands. Further research is needed to determine whether the CS is truly necessary for delivery of high quality care and whether the CS is a necessary addition to the guidelines of the individual professionals groups. The studies among patients underline the importance of mastery in relation to patients' perceived autonomy support from their primary caregiver, self-management skills and health-related quality of life. Furthermore, autonomy support and motivational interviewing, together with strategies for enhancing perceived competence, are important elements to include in interventions aimed at improving adherence to self-care behaviors and enhancing quality of life. Altogether, diabetes care has undergone several changes aimed to improve the quality of care. The experiences with the implementation of the CS for diabetes and the NAD underline the beneficial value of the ongoing process in which healthcare moves towards an integrated multidisciplinary approach of chronic diseases worldwide which incorporates personalized care that fits the patients' needs and characteristics. 


\section{Samenvatting}

Diabetes is een snelgroeiend gezondheidsprobleem met 382 miljoen patiënten wereldwijd. In 2011 telde Nederland 834.100 mensen met diabetes en in datzelfde jaar kwamen er ongeveer 87.000 nieuwe patiënten bij. Diabetes is een multifactorieel probleem en vereist een multidisciplinaire aanpak in preventie en behandeling. De Nederlandse gezondheidzorg staat, net zoals de zorg in de meeste geïndustrialiseerde landen, voor de uitdaging om continuïteit en kwaliteit van zorg voor het groeiend aantal mensen met diabetes te waarborgen. De laatste jaren zijn er daarom binnen de diabeteszorg veel ontwikkelingen in gang gezet. Een van deze ontwikkelingen betreft de introductie en implementatie van de Nederlandse Diabetes Federatie (NDF) Zorgstandaard (ZS) voor diabetes. De ZS beschrijft de norm waaraan goede diabeteszorg moet voldoen en volgens welke richtlijnen de behandeling van mensen met diabetes moet worden vormgegeven. Nederland is uniek in het gebruik van de ZS. In 2009 startte het NDF het Nationaal Actieprogramma Diabetes (NAD) met een subsidie van het ministerie van VWS. Het belangrijkste doel van het NAD was de landelijke implementatie van de ZS (Nederlandse Diabetes Federatie, 2009).

Het doel van dit proefschrift was om meer inzicht geven in de diabeteszorg en de introductie van de ZS vanuit het perspectief van zorgprofessionals en diabetes patiënten. Vanuit het perspectief van zorgprofessionals heeft dit proefschrift hun percepties ten aanzien van de inhoud en implementatie van de ZS en ervaren bevorderende en belemmerende factoren in de diabeteszorg onderzocht. Vanuit het perspectief van patiënten is onderzocht hoe concepten uit de Self Determination Theory en mastery geassocieerd waren met zelf-management, algemene controle over diabetes en kwaliteit van leven van Nederlandse type 1 en type 2 patiënten.

Na een algemene inleiding in hoofdstuk 1, worden twee studies uitgevoerd vanuit het perspectief van zorgprofessionals beschreven (hoofdstuk 2 en 3), gevolgd door twee studies vanuit het perspectief van diabetespatiënten (hoofdstuk 4 en 5). In hoofdstuk 6 
en 7 worden twee studies vanuit een geïntegreerd perspectief gepresenteerd. Hoofdstuk 8 eindigt met een algemene discussie.

\section{Perspectief van zorgprofessionals}

Hoofdstuk 2 beschrijft een cross-sectioneel vragenlijstonderzoek onder Nederlandse zorgprofessionals ( $\mathrm{N}=1547)$. In dit onderzoek zijn de percepties van zorgprofessionals ten aanzien van de ZS diabetes en ervaren belemmeringen in kaart gebracht. Een beperkt percentage zorgprofessionals had de ZS in bezit. Bijna twee derde van de professionals die in zekere mate bekend waren met de ZS was van mening dat de ZS bijdraagt aan het waarborgen van de kwaliteit van de zorg. Verder werd de haalbaarheid van het werken conform de ZS werd door de meerderheid positief beoordeeld. Echter, professionals waren geneigd de richtlijnen van de individuele beroepsgroepen meer als norm voor goede zorg te beschouwen dan de ZS. Een minderheid van de professionals die in zekere mate bekend waren met de ZS gaf aan volledig conform de ZS te werken. De belangrijkste belemmering in relatie tot de toepassing van de ZS was het gebrek aan (toegang tot) effectieve leefstijlinterventies om zorg te leveren aan mensen met (een verhoogd risico op) diabetes. Een andere belangrijke belemmering was gerelateerd aan financiële aspecten, wet- en regelgeving bij verlening van zorg en preventie volgens de ZS. Deze belemmeringen verdienen aandacht om het werken conform de ZS te verbeteren.

Hoofdstuk 3 presenteert een kwalitatieve studie onder 18 zorgprofessionals die geïnterviewd werden om ervaren bevorderende en belemmerende factoren in de diabeteszorg in kaart te brengen met behulp van het Chronic Care Model (CCM). Een van de belangrijkste bevorderende factoren was de meer prominente rol van praktijkondersteuners en diabetesverpleegkundigen in de diabeteszorg als resultaat van substitutie van zorg. Andere gerapporteerde bevorderende factoren waren de ZS zelf en multidisciplinaire samenwerking, hoewel er nog steeds ruimte voor verbetering is in de samenwerking met bepaalde beroepsgroepen, namelijk met diëtisten, fysiotherapeuten en apothekers, en tussen professionals werkzaam in de eerste en tweede lijn. Verder werd benchmarking als bevorderend ervaren; echter de kwaliteit van de indicatoren ten behoeve van benchmarking werden door de meerderheid als belemmering gerapporteerd. Belangrijke belemmeringen in relatie tot het gezondheidszorg systeem waren de DBC- systematiek voor de financiering van diabeteszorg, de rol van zorgverzekeraars en het gebrek aan motivatie bij patiënten. Een andere belangrijke belemmering onder zorgprofessionals was het gebrek aan (toegang tot) effectieve leefstijlprogramma's en preventie initiatieven voor diabetespatiënten. Dit wijst op de meerwaarde van de toepassing van het Expanded CCM. 


\section{Perspectief van diabetes patiënten}

Hoofdstuk 4 beschrijft een cross-sectionele vragenlijst studie waarin onderzocht is hoe waargenomen autonomie ondersteuning, ervaren competentie en zelfregulatie ten aanzien van de behandeling van Nederlandse type $1(\mathrm{~N}=143)$ en type 2 patiënten $(\mathrm{N}=384)$ geassocieerd zijn met diabetes zelfzorg activiteiten (gezond dieet, fysieke activiteit, monitoren van bloedglucose waarden en medicatie gebruik) en algemene diabetes controle. De meeste deelnemers voelden zich competent, ondersteund in hun autonomie en gaven aan hun diabetes op een autonome manier zelf te kunnen reguleren. De resultaten bevestigen het belang van ervaren competentie bij type 1 en type 2 patiënten in relatie tot het volgen van een gezond dieet en algemene diabetes controle. De bevindingen van deze studie benadrukken ook de behoefte aan autonomie ondersteunende zorgverleners in de diabeteszorg. Opvallend is dat bij type 1 patiënten de invloed van waargenomen autonomie ondersteuning op diabetes controle gemedieerd lijkt te worden door ervaren competentie, terwijl type 2 patiënten een directe associatie tussen autonomie ondersteuning en diabetes controle aanwezig blijft.

Hoofdstuk 5 presenteert een cross-sectioneel vragenlijstonderzoek onder 3352 Nederlandse patiënten met diabetes type 2 . Het doel van deze studie was het onderzoeken van de associatie tussen mastery (grip hebben op het leven ) en ervaren autonomie ondersteuning op zelfmanagement en kwaliteit van leven. Patiënten hadden relatief hoge scores op waargenomen autonomie ondersteuning en zelfmanagement en rapporteerden een relatief lage mate van mastery te ervaren. Patiënten met meer fysieke en psychologische complicaties hadden significant lagere scores op mastery, waargenomen autonomie ondersteuning, zelfmanagement en kwaliteit van leven. Patiënten met een hogere BMI rapporteerden een lager niveau van mastery. De bevindingen benadrukken het belang van een autonomie ondersteunende counseling stijl van zorgverleners in de diabeteszorg. Daarnaast bevestigen de resultaten het belang van mastery in relatie tot waargenomen autonomie ondersteuning, zelfmanagement vaardigheden en kwaliteit van leven.

Hoofdstuk 6 beschrijft twee cross-sectionele studies uitgevoerd onder zorgprofessionals en patiënten met type 1 en type2 diabetes in 2010 en 2013. Het belangrijkste doel van deze studies was het bepalen van de bekendheid met en waardering en implementatie van de ZS diabetes onder zorgprofessionals. Daarnaast had dit hoofdstuk als doel de resultaten van beide studies te vergelijken. In 2013 waren zorgprofessionals significant vaker bekend met de ZS in vergelijking met 2010 en rapporteerden ze vaker volledig conform de ZS te werken. De meerderheid had een positieve houding ten aanzien van de ZS en de bijdrage die de ZS levert aan het waarborgen van de kwaliteit van de zorg. Over het algemeen waren patiënten tevreden over de contacten met hun zorgverlener(s) en rapporteerden in hoge mate betrokken te worden in hun behandeling. Aangezien de ZS voor diabetes als eerste ontwikkeld en geïmplementeerd is, kunnen de ervaringen met 
deze standaard gebruikt worden bij de implementatie van andere zorgstandaarden voor chronische aandoeningen. Een volgende stap kan zijn de zorg op lokaal niveau te implementeren en in te bedden in een wijkgerichte aanpak waarin ook aandacht is voor preventie van andere chronische aandoeningen.

Hoofdstuk 7 had als doel inzicht te geven in de implementatie van het Nationaal Actieprogramma Diabetes (NAD) in de afgelopen tien jaar, met de introductie van de ZS diabetes als belangrijkste doel en bestaande uit een specifiek implementatieplan en een pilot in een aantal regio's. Het NAD en de introductie van de ZS kunnen als voorbeeld fungeren voor vergelijkbare benaderingen in andere landen. Over het algemeen zijn positieve veranderingen gevonden in de periode 2010-2013. Een vergelijking van de resultaten in de pilot regio's met die in de rest van Nederland laat zien dat professionals in deze regio's significant beter scoorden op implementatie en waardering van de ZS. Deze positieve trend is ook terug te zien in de hoge mate van tevredenheid onder patienten en hun betrokkenheid in de behandeling. Professionals die de ZS in bezit hadden, scoorden significant beter op de implementatie van diverse elementen van de ZS dan professionals die de ZS niet in bezit hadden. Terugkijkend op het NAD lijkt het erop dat de ZS heeft gefungeerd als een vliegwiel om processen ter verbetering van de zorg voor chronisch zieken in Nederland vorm te geven. Ervaringen met het NAD en de ZS onderschrijven de behoefte aan een geïntegreerde multidisciplinaire aanpak van de zorg voor diabetes.

Hoofdstuk 8 presenteert een samenvatting en reflectie van de belangrijkste bevindingen van de studies gepresenteerd in dit proefschrift. Dit hoofdstuk beschrijft ook de methodologische overwegingen ten aanzien van het onderzoeksdesign, de onderzoekspopulatie, de meetinstrumenten en statistische analyses en de sterke punten van de studies. Daarna gaat het hoofdstuk in op de implicaties voor praktijk, theorie en verder onderzoek. De algemene conclusie is dat het NAD geresulteerd heeft in inbedding van de ZS in de Nederlandse gezondheidszorg. De introductie van de ZS, ondersteund door het $\mathrm{Na-}$ tionaal Actieprogramma Diabetes, en samen met de DBC-systematiek als parallelle ontwikkeling, als vliegwiel heeft gefungeerd voor het realiseren van verschillende faciliterende processen gerelateerd aan de bredere implementatie van zorgstandaarden ter verbetering van de zorg voor mensen met een chronische aandoening in Nederland. Verder onderzoek is nodig om te bepalen of de ZS echt noodzakelijk is voor het leveren van kwalitatief goede zorg en essentieel is als aanvulling op de richtlijnen van de individuele beroepsgroepen. De studies onder patiënten bevestigen het belang van mastery bij patiënten in relatie tot de waargenomen autonomie ondersteuning van hun primaire zorgverlener, zelfmanagement vaardigheden en kwaliteit van leven. Daarnaast zijn autonomie ondersteuning en motiverende gespreksvoering, samen met strategieën voor het verbeteren van ervaren competenties van patiënten belangrijke elementen om op te 
nemen in toekomstige interventies gericht op het verbeteren van zelfmanagement en kwaliteit van leven van diabetes patiënten.

Samengevat heeft de diabeteszorg diverse veranderingen ondergaan gericht op het verbeteren van de Kwaliteit van de zorg. De ervaringen met de implementatie van de ZS diabetes en het NAD bevestigen de meerwaarde van het lopende proces waarin de gezondheidszorg toegaat naar een geïntegreerde multidisciplinaire aanpak van chronische ziekten waarin gepersonaliseerde zorg die aansluit op de behoeften en kenmerken van de patiënten centraal staat. 



\section{Dankwoord}

Ik had dit proefschrift niet kunnen voltooien zonder de hulp en steun van veel mensen. Graag wil ik dan ook iedereen bedanken die op enige wijze heeft bijgedragen aan het tot stand komen van dit proefschrift. Een aantal mensen wil ik in het bijzonder noemen.

Allereerst een bijzonder woord van dank voor mijn promotoren. Stef, bedankt voor je dagelijkse begeleiding in de afgelopen jaren! Jouw optimisme en enthousiasme voor het vak hebben me elke keer opnieuw weten te stimuleren om het beste uit mezelf te halen. Bedankt ook voor de vrijheid en het vertrouwen dat je me hebt gegeven, en voor je snelle reacties op de stukken in de eindfase. Nanne, bedankt voor je begeleiding, het delen van je expertise en je kritische blik. Je hebt me goed geholpen om alles in breder perspectief te plaatsen.

Marloes, ook een speciaal woord van dank voor jou. De samenwerking met en begeleiding vanuit ResCon waren van enorme meerwaarde voor dit project. Ik kijk daar met heel veel plezier op terug! Jouw optimisme en relativeringsvermogen tijdens stressvolle pieken hebben me enorm geholpen alles tot een goed einde te brengen. Dank ook voor het meeschrijven aan alle rapportages en artikelen. Arlette, ook jij bedankt voor de fijne samenwerking, je kritische blik en het meeschrijven aan de rapportages en artikelen. Patricia, jou wil ik bedanken voor de kansen die je me hebt gegeven. Ik heb met heel veel plezier met je samengewerkt en wat fijn dat we alle lopende voedingsartikelen nu hebben kunnen afronden.

Verder wil ik graag de leescommissie, Dike van de Mheen, Lea Maes, Trudy van der Weijden, Dries Hettinga en Ciska Hoving, bedanken voor de tijd om mijn proefschrift te beoordelen.

Daarnaast een woord van dank voor alle zorgprofessionals en patiënten die deelgenomen hebben aan de studies. Zonder jullie medewerking had ik dit proefschrift niet kunnen schrijven! 
Ook wil ik de Nederlandse Diabetes Federatie en het Diabetes Fonds bedanken voor de prettige samenwerking in de afgelopen jaren. Inge bedankt voor het delen van je expertise en het meeschrijven aan de artikelen.

Mijn oud-collega's van Gezondheidsbevordering wil ik bedanken voor de leuke tijd; en een aantal personen in het bijzonder. Kathelijne, bedankt voor de fijne samenwerking op de verschillende projecten in de afgelopen jaren en voor alle gezelligheid. Het was heel fijn een collega als jou te hebben! Jessica, ik had me geen fijner kamergenootje bij GB kunnen wensen! Bedankt voor de fijne werksfeer en de leuke tijd. Ik mis onze gesprekken, die 9 van de 10 keer toch op voeding uitliepen, wel hoor! Femke, bedankt voor je ondersteuning bij het project en de fijne samenwerking. Dorus bedankt voor het meeschrijven aan de parenting papers en het afronden van het laatste artikel. Kimberly en Renee, kamergenootjes tijdens de laatste loodjes, bedankt voor de fijne sfeer. En natuurlijk Marja, Leon en Patricia, bedankt voor het beantwoorden van vele vragen en de ondersteuning bij alle praktische zaken die de afgelopen jaren voorbij zijn gekomen.

Ook wil ik oud-collega's van ResCon bedanken. Het was fijn om regelmatig bij jullie op kantoor te werken en in rust te kunnen schrijven; zeker in de eindfase! Annemarie, bedankt voor alle gezelligheid en de fijne gesprekken. Ruud, bedankt voor je inspanningen bij de opstart van het project. Lotje, bedankt voor de prettige samenwerking in de eerste fase.

Collega's van het IVO, bedankt voor jullie betrokkenheid en de fijne werksfeer. Wat leuk om nu alweer een tijdje bij jullie aan het werk te zijn! Elske, Dike en Miranda bedankt voor de kansen die jullie me hebben gegeven en voor de flexibiliteit rondom het afronden van mijn proefschrift.

Lieve vrienden, bedankt voor jullie steun, begrip en alle gezelligheid. Anne, wat bijzonder dat we al zo lang bevriend zijn, alles kunnen delen en alle levensfasen samen meemaken! Niels bedankt voor de leuke speeluurtjes: een welkome afwisseling op het harde werken in de eindfase! Dorieke, wat fijn dat we al zo lang vriendinnen zijn en al die jaren al zoveel hebben gedeeld samen! Bedankt voor je luisterende oor, de gezellige theetjes, lunches, dubbel dates en de bezoekjes aan kleine Hugo. Mijn paranimfen Mandy en Sanne: ik vind het heel speciaal dat jullie vandaag naast me staan! Mandy, wat hebben we een fijne vriendschap. Bedankt voor alle leuke momenten samen! Sanne, ik vond het bijzonder om samen als vriendinnen en collega's te promoveren. Bedankt voor die leuke tijd! Jill en Dionne, wat fijn dat we al sinds onze studietijd bevriend zijn en zoveel leuke momenten gedeeld hebben! Aniek, Elke en Laure, bedankt voor alle gezelligheid! 
Verder ook dank aan mijn familie die er altijd zijn als ik ze nodig heb.

Vivianne en Jan, wat fijn om alles met jullie te kunnen delen en wat hebben we het altijd gezellig! En Vivi, bedankt dat ik elke keer een beroep mag doen op jouw grenzeloze creativiteit. Ik ben erg blij met de mooie kaft!

Fred en Lieke, ik had me geen fijnere schoonouders kunnen wensen. Ik kijk met veel plezier terug op mijn logeerpartijen in jullie B\&B tijdens het laatste jaar. Bedankt voor de lekkere etentjes, goede zorgen, jullie luisterend oor en goede adviezen. Bij jullie zijn is altijd heerlijk ontspannend!

Rob en Jaimy, Moniek en Kevin, ik had me geen betere (schoon)broer en (schoon)zus kunnen wensen! Bedankt dat jullie er altijd voor me zijn en voor de fijne band die we hebben! Moniek, wat fijn om alles met mijn zusje te kunnen delen.

Lieve pap en mam, dankzij jullie liefde, steun en vertrouwen sta ik hier vandaag. Jullie hebben me altijd gestimuleerd alles uit mezelf te halen. Dank voor jullie betrokkenheid, jullie luisterend oor, alle telefoontjes en mailtjes en het gezellige samenzijn. Wat fijn dat jullie altijd voor me klaar staan. Bedankt voor alles!

Lieve Maurice, wat vind ik het heerlijk om elke dag weer bij jou thuis te komen: mijn rustpunt. En wat bijzonder om nu als mevrouw Buteijn door het leven te gaan! Bedankt voor je onvoorwaardelijke liefde, je geduld en relativerende gekkigheid: you're just to good to be true! Laten we samen nog meer genieten van elke dag en met veel plezier uitkijken naar alles wat het leven ons nog gaat brengen!

Lieke 



\section{Curriculum Vitae}

Lieke Geertruda Maria Raaijmakers was born on February 1st, 1986 in Geldrop, the Netherlands. After completing secondary school (Atheneum) at the Carolus Borromeus College in Helmond in 2004, she started studying Health Sciences at Maastricht University. Her specializations were Health Education and Promotion and Movement Sciences. During her studies, she was an exchange student at the Faculty of Sports and Health Sciences, University of Yväskylä, Finland. In 2007, Lieke obtained her BSc degree, where after she started studying the Master of Public Health, with a specialization in Health Education and Promotion. Parallel to her internship at the Department of Health Promotion, Lieke worked there as a student assistant. After graduating, Lieke started working as a research assistant at the Department of Health Promotion in August 2008. In April 2010 she started working as a PhD student at the same department. As of May 2014, Lieke is working as a researcher at the IVO Addiction Research Institute in Rotterdam. 



\section{Publication list}

\section{Publications from this dissertation}

Raaijmakers, L.G.M., Kremers, S.P.J., Schaper, N.C., de Weerdt, I., Martens, M.K., de Vries, N.K. The implementation of the National Action program Diabetes in the Netherlands: lessons learned. (under review)

Raaijmakers, L.G.M., Martens, M.K., Bagchus, C., de Vries, N.K., Kremers, S.P.J. Correlates of perceived self-care activities and diabetes control among Dutch type 1 and type 2 diabetics. (resubmitted)

Raaijmakers, L.G.M., Martens, M.K., Hesselink, A.E., de Weerdt, I., de Vries, N.K., Kremers, S.P.J. Mastery and perceived autonomy support are correlates of selfmanagement and quality of life among Dutch diabetes patients. Patient Education and Counseling, 97(1), 75-81.

Raaijmakers, L.G.M., Martens, M.K., Bagchus, C., de Vries, N.K., Kremers, S.P.J. (2013) Perceptions of Dutch health care professionals regarding the Care Standard for diabetes. BMC Research Notes, 17(6), 417.

Raaijmakers, L.G.M., Hamers, F.J.M., Martens, M.K., Bagchus, C., de Vries, N.K., Kremers, S.P.J. (2013). Perceived facilitators and barriers in diabetes care: a qualitative study among health care professionals in the Netherlands. BMC Family Practice, 14(1), 114.

Raaijmakers, L.G.M., Bagchus, C., Martens, M.K., Jonkers, R., Kremers, S.P.J. (2011). De implementatie van de NDF Zorgstandaard Diabetes: Onderzoek onder zorgprofessionals en patiënten in het kader van het Nationaal Actieprogramma Diabetes. Nederlands tijdschrift voor Diabetologie, 9(4), 167-174. 
Raaijmakers, L.G.M., Martens, M.K., Hesselink, A.E., de Weerdt, I., Kremers, S.P.J. (2011). De implementatie van de NDF Zorgstandaard Diabetes anno 2013. Nederlands tijdschrift voor Diabetologie, 11(3): 104-109.

\section{Additional publications}

Gevers, D., Raaijmakers, L.G.M., Bessems, K.M.H.H., Teuscher, D., Kremers, S.P.J., van Assema, P. Restrictive rules of Dutch mothers on their children's dietary intake between meals. (submitted)

Gubbels, J.S., Raaijmakers, L.G.M., Gerards, S.M.P.L., Kremers, S.P.J. (2014). Dietary intake in Dutch 1- to 3-year-old children at childcare and at home. Nutrients, 6(1), 304318.

Raaijmakers, L.G.M., Gevers, D., Teuscher, D., Kremers, S.P.J., van Assema, P. (2014). Emotional and instrumental feeding practices of Dutch mothers towards foods eaten between main meals. BMC Public Health, 14, 171.

Bessems, K.M.H.H., van Assema, P., Raaijmakers, L.G.M. (2013). Evaluatie van een online actieplanprogramma over fruit, ontbijt en tussendoortjes voor leerlingen van het VMBO. Tijdschrift voor Gezondheidswetenschappen, 91(4), 195-198.

Bessems, K.M.H.H., van Assema, P.,Martens, M.K., Paulussen, T.G.W.M., Raaijmakers, L.G.M., de Rooij, M., de Vries, N.K. (2013). Healthier food choices as a result of the revised healthy diet programme Krachtvoer for students of prevocational schools. International Journal of Behavioral Nutrition and Physical Activity, 9, 60.

Raaijmakers, L.G.M., Bessems, K.M.H.H., Kremers, S.P.J., van Assema, P. (2012). Energy, saturated fat and fibre intakes among Dutch children and adolescents at breakfast and implications for educational messages. Nutrition and Health, 21(4), 219-231.

Helmink, J.H.M, Raaijmakers, L.G.M., Rutten, G.M., de Vries, N.K., Kremers, S.P.J. (2012). Gecombineerde leefstijl interventies in Nederland: ervaringen uit de BeweegKuur. Tijdschrift voor Gezondheidswetenschappen, 91(2), 89- 91.

Bessems, K.M.H.H., van Assema, P., Martens, M.K., Paulussen, T.G.W.M., Raaijmakers, L.G.M., de Vries, N.K. (2011). Appreciation and implementation of the Krachtvoer healthy diet promotion programme for 12- to 14- year-old students of prevocational schools. BMC Public Health, 11, 909. 
Raaijmakers, L.G.M., Bessems, K.M.H.H., Kremers, S.P.J., van Assema, P. (2010). Breakfast consumption among children and adolescents in the Netherlands. European Journal of Public Health, 20(3), 318-324.

\section{Reports}

Raaijmakers, L.G.M., Martens, M.K, Hesselink, A.E., Kremers, S.P.J. (2013). NAD monitor 2013. Onderzoek onder zorgprofessionals en diabetespatiënten, Universiteit Maastricht.

Raaijmakers, L.G.M., Helmink, J.H.M., Hamers, F.J.M., Kremers, S.P.J. (2012). Inhoudelijke monitor lokale netwerken, Universiteit Maastricht.

Raaijmakers, L.G.M., Helmink, J.H.M., Hamers, F.J.M., Kremers, S.P.J. (2012). Implementatie en continuering van de BeweegKuur: Monitorstudie onder zorgverleners, najaar 2011. Universiteit Maastricht.

Raaijmakers, L.G.M., Bagchus, C., Martens, M.K., Jonkers, R., Kremers, S.P.J. (2011). NAD monitor 2010. Onderzoek onder zorgprofessionals en diabetespatiënten, Universiteit Maastricht. 